\title{
Risk-Based Operation and Maintenance of Offshore Wind Turbines
}





\title{
Risk-Based Operation and Maintenance of Offshore Wind Turbines
}

\author{
Revised Version \\ PhD Thesis \\ Defended in public at Aalborg University \\ April 22, 2013 \\ Jannie Sønderkær Nielsen \\ Department of Civil Engineering, \\ The Faculty of Engineering and Science, \\ Aalborg University, Aalborg, Denmark
}

River Publishess

Aalborg 
ISBN 978-87-93102-46-0 (e-book)

Published, sold and distributed by:

River Publishers

Niels Jernes Vej 10

9220 Aalborg Ø

Denmark

Tel.: +45369953197

www.riverpublishers.com

Copyright for this work belongs to the author, River Publishers have the sole right to distribute this work commercially.

All rights reserved (c) 2013 Jannie Sønderkær Nielsen.

No part of this work may be reproduced, stored in a retrieval system, or transmitted in any form or by any means, electronic, mechanical, photocopying, microfilming, recording or otherwise, without prior written permission from the Publisher. 


\section{Preface}

The work presented in this dissertation is part of the project "Reliabilitybased analysis applied for reduction of cost of energy for offshore wind turbines", supported by the Danish Council for Strategic Research, grant no. 2104-08-0014. The financial support is greatly appreciated. The work was carried out at the Department of Civil Engineering, Aalborg University, Denmark.

\section{Acknowledgements}

First and foremost, I would like to thank my supervisor Professor John Dalsgaard Sørensen for dedicated supervision and for an always positive attitude. Secondly, I would like to thank the people at the wind energy unit at ECN, where I stayed for three months during the $\mathrm{PhD}$ studies. I definitely learned a lot about real life decision problems, about working with condition monitoring data, and about their approaches for assessing maintenance costs. Also thanks to our contacts from DONG Energy, especially Frederik Ettrup Brink for sharing his knowhow and for helping with condition monitoring data.

Finally, I would like to thank my family, friends and colleges for their support, especially my husbond, who is probably now the bioinformatician knowing most about risk-based operation and maintenance for wind turbines. 



\section{Thesis title:}

Risk-based operation and maintenance of offshore wind turbines

\section{PhD student:}

Jannie Sønderkær Nielsen (previously Jannie Jessen Nielsen)

\section{Supervisor:}

Prof. John Dalsgaard Sørensen

\section{List of published papers:}

Papers included in the appendix of the thesis:

1. Nielsen, J. J. and J. D. Sørensen. 2011. "On Risk-Based Operation and Maintenance of Offshore Wind Turbine Components." Reliability Engineering \& System Safety 96 (2011): 218-229. DOI: 10.1016/j.ress.2010.07.007.

2. Nielsen, J. J. and J. D. Sørensen. 2010. "Planning of O\&M for Offshore Wind Turbines using Bayesian Graphical Models." ESREL 2010 annual conference, Rhodes, Greece.

3. Nielsen, J. J. and J. D. Sørensen. 2011. "Risk-Based Operation and Maintenance of Offshore Wind Turbines using Bayesian Networks." 11th International Conference on Applications of Statistics and Probability in Civil Engineering, ICASP11, ETH, Zurich, Switzerland.

4. Nielsen, J. J. and J. D. Sørensen. 2011. "Challenges for Risk-based Maintenance Planning for Offshore Wind Turbines." 21st International Offshore and Polar Engineering Conference, ISOPE 2011, Maui, Hawaii, USA.

5. Nielsen, J. S. and J. D. Sørensen. 2012. "Maintenance Optimization for Offshore Wind Turbines using POMDP." IFIP WG 7.5 Working Conference on Reliability and Optimization of Structural Systems, AUA, Yerevan, Armenia.

6. Nielsen, J. S., R. P. v.d. Pieterman and J. D. Sørensen. 2013. "Analysis of pitch system data for condition monitoring." Wind Energy (Published online). DOI: 10.1002/we.1586.

Other papers:

7. Nielsen, J. J. and J. D. Sørensen. 2009. "Risk-Based Operation and Maintenance of Offshore Wind Turbines." 5th PhD Seminar on Wind Energy in Europe, EAWE, DU, Durham, UK. 
8. Nielsen, J. J. and J. D. Sørensen. 2010. "Risk-Based Operation and Maintenance Planning for Offshore Wind Turbines." IFIP WG 7.5 Working Conference on Reliability and Optimization of Structural Systems, TUM, Munich, Germany.

9. Nielsen, J. J. and J. D. Sørensen. 2010. "Risk Based Maintenance of Offshore Wind Turbines Using Bayesian Networks." 6th EAWE PhD Seminar on Wind Energy in Europe, NTNU, Trondheim, Norway.

10. Nielsen, J. J. and J. D. Sørensen. 2010. "Bayesian Networks as a Decision Tool for O\&M of Offshore Wind Turbines." 5th International ASRANet Conference, Integrating Structural Analysis, Risk \& Reliability, Edinburgh, UK.

11. Nielsen, J. J. and J. D. Sørensen. 2011. "Risk-Based Operation and Maintenance Using Bayesian Networks: influence of epistemic uncertainties." 24th International Congress on Condition Monitoring and Diagnostic Engineering Management (COMADEM2011): advances in industrial asset integrity management, Stavanger, Norway.

12. Nielsen, J. J. and J. D. Sørensen. 2011. "Analysis of Pitch Gear Deterioration using Indicators.", EWEA Offshore 2011, Amsterdam, The Netherlands.

13. Sørensen, J. D. and J. J. Nielsen. 2011. "Risk-based operation and maintenance for offshore wind turbines.", EWEA Offshore 2011, Amsterdam, The Netherlands.

This thesis has been submitted for assessment in partial fulfillment of the $\mathrm{PhD}$ degree. The thesis is based on the submitted or published scientific papers which are listed above. Parts of the papers are used directly or indirectly in the extended summary of the thesis. As part of the assessment, co-author statements have been made available to the assessment committee and are also available at the Faculty. The thesis is not in its present form acceptable for open publication but only in limited and closed circulation as copyright may not be ensured. 


\section{Summary}

For offshore wind turbines, maintenance costs are substantial due to a large number of component failures. In addition to the repair costs, equipment costs and lost production costs are large due to limited accessibility to the turbines. In order to reduce the costs, preventive methods can be used, but presently maintenance is not planned using advanced methods taking all available information into account in a consistent manner.

Maintenance decisions can be made based on risk-based methods, where the total expected life cycle costs are minimized. Methods have been developed for assessing the corrective maintenance costs, and many studies have also been published for preventive maintenance. However, an important part is the modeling of the relationship between maintenance and reliability, which is typically not modeled in a consistent way. Therefore, the methods cannot be used directly for optimal maintenance planning, taking into account monitoring, inspections, preventive repairs and corrective repairs. This can be done using methods developed from a Bayesian perspective. Such methods have been developed for use in the oil and gas industry under the name Risk-Based Inspection (RBI), and are based on the Bayesian pre-posterior decision analysis. In order to make the problem computationally feasible, approximations are necessary. The approach typically used for RBI cannot directly be transferred to wind turbines as the conditions are different. For wind turbine components, the reliability is lower compared to oil and gas structures. In addition, condition monitoring systems are often available, and the information should be taken into account when making decisions.

In this thesis, methods for risk-based maintenance planning using Bayesian methods are investigated, with the aim of making optimal decisions considering all available information. First, a theoretical damage model is formulated, the model is then updated using condition monitoring data, and the updated model is used as basis for risk-based decision making. Several approaches for solving the decision problems have been considered: various types of stationary decision rules, limited memory influence diagrams, and Markov decision processes. Several of the approaches are used together with Bayesian networks.

The approaches have been tested for theoretical decision problems, and 
the expected costs were compared for different strategies. It was possible to achieve big savings, but the results of course largely depend on the input. With real decision problems, it can be difficult to formulate the model if the design assumptions are not fulfilled in real life. The potential of the method is large, but further development is necessary, especially concerning the modeling of specific examples. 


\section{Summary in Danish}

For havvindmøller er omkostningerne til vedligehold omfattende da der ofte er komponenter i vindmøllen, som svigter. Udover reparationsomkostningerne er der store omkostninger forbundet med leje af materiel og tabt produktion grundet begrænset tilgængelighed til vindmøllerne. For at reducere omkostningerne kan der benyttes præventive metoder, men i dag benyttes der imidlertid normalt ikke avancerede metoder, der tager højde for al tilgængelig information på en konsistent måde, når præventiv vedligehold planlægges.

Beslutninger om vedligehold kan træffes ved anvendelse af risikobaserede metoder, hvor de totale forventede omkostninger i levetiden minimeres. Der er udviklet metoder til at estimere omkostningerne til korrektiv vedligehold, og ligeledes er der publiceret mange studier vedrørende præventiv vedligehold. En vigtig del er modelleringen af sammenhængen mellem vedligehold og pålidelighed, hvilket ofte ikke er modelleret på en konsistent måde. Metoderne kan derfor ikke bruges direkte til optimal planlægning af drift og vedligehold, hvor der tages højde for information fra overvågningssystemer, inspektioner, præventive reparationer samt korrektive reparationer. Dette kan gøres ved at benytte metoder, som er udviklet fra et Bayesiansk perspektiv. Den slags metoder er allerede udviklet til brug i olie- og gasindustrien under navnet Risikobaseret Inspektionsplanlægning (RBI), og er baseret på Bayesiansk preposteriori beslutningsanalyse. For at gøre løsningen beregningsteknisk muligt er det nødvendigt at indføre approksimationer. Den tilgangsvinkel, som typisk benyttes til RBI, kan ikke overføres direkte til vindmøller, da forholdene er anderledes. For vindmøllekomponenter er pålideligheden mindre sammenlignet med olie- og gaskonstruktioner. Desuden er der ofte overvågningssystemer på vindmøller, og informationerne derfra bør medtages, når der træffes beslutninger.

I denne afhandling benyttes Bayesianske metoder til risikobaseret vedligeholdelsesplanlægning med det formål at træffe optimale beslutninger, der tager højde for al tilgængelig information. Først formuleres en teoretisk skadesmodel, hvorefter modellen opdateres ved hjælp af overvågningsdata, og endelig bruges den opdaterede model som basis for risikobaseret beslutningstagning. Flere fremgangsmåder til at løse beslutningsproblemerne er blevet betragtet: forskellige slags stationære beslutningsregler, influensdia- 
grammer med begrænset hukommelse (LIMID), og Markov beslutningsprocesser. Ved flere af fremgangsmåderne er Bayesianske netværk blevet brugt til beregningerne.

Fremgangsmåderne er blevet afprøvet med teoretiske beslutningsproblemer, og de forventede omkostninger blev sammenlignet for forskellige strategier. Det var muligt at opnå store besparelser, men resultaterne afhænger naturligvis meget af forudsætningerne. For virkelige beslutningsproblemer kan det være vanskeligt at formulere modellen, hvis forudsætningerne brugt under dimensioneringen ikke er opfyldt i virkeligheden. Metoderne har store potentialer, men kræver yderligere udvikling, specielt med hensyn til at modellere virkelige eksempler. 


\section{Contents}

1 Introduction $\quad 3$

1.1 Cost of wind energy . . . . . . . . . . . . . . 3

1.1.1 Costs of O\&M . . . . . . . . . . . 4

1.1.2 Failure statistics . . . . . . . . . . . . 5

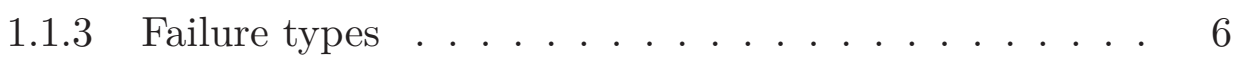

1.2 Maintenance planning . . . . . . . . . . . . . 6

1.2.1 Preventive maintenance . . . . . . . . . . . 8

1.2.2 Condition monitoring . . . . . . . . . . . . . 8

1.2.3 Maintenance decisions . . . . . . . . . . . . . . . . . . . . . 9

1.2.4 Costs and consequences . . . . . . . . . . . . . 9

1.2.5 Current maintenance practice . . . . . . . . . 10

1.3 State-of-the-art for planning of maintenance for offshore wind turbines ........................ 11

1.3.1 Estimation of costs to corrective maintenance . . . . 11

1.3.2 Decisions on preventive maintenance . . . . . . . . . 14

1.3.3 Conclusions on state-of-the-art . . . . . . . . . 19

1.4 Pre-posterior decision analysis . . . . . . . . . . . . . 19

1.4.1 Risk-Based Inspection . . . . . . . . . . . . . 20

1.5 Objective of thesis . . . . . . . . . . . . . 22

1.5.1 Approach ................... 22

2 Deterioration modeling 25

2.1 Fatigue design models . . . . . . . . . . . . . . 26

2.1.1 SN curves . . . . . . . . . . . . . . . 27

2.1.2 Palmgreen-Miner damage accumulation law . . . . . 27

2.1.3 Fatigue for wind turbine components . . . . . . . . . 29

2.2 Fatigue models with measurable damage size . . . . . . . . 31

2.2.1 Fracture mechanical model . . . . . . . . . . 31

2.2.2 Calibration of SN model to FM model . . . . . . . . . 32

3 Updating of deterioration model 33

3.1 Indicator models . . . . . . . . . . . . . . . . . . . . . . . . . . . 33

3.2 Bayesian updating ................... 34 
3.3 Bayesian networks . . . . . . . . . . . . . . 34

3.3.1 Conditional independence . . . . . . . . . . . . . 35

3.3.2 Sampling based inference . . . . . . . . . . . . . 36

3.3.3 Inference in discrete Bayesian networks . . . . . . . 37

3.3.4 Dynamic Bayesian networks . . . . . . . . . . . . 38

3.4 Application of $\mathrm{BN}$ to deterioration modeling . . . . . . . . 40

3.4 .1 Continuous . . . . . . . . . . . . . . . . 41

3.4 .2 Discrete . . . . . . . . . . . . . . . . 42

4 Risk-based decision making 45

4.1 Methods with fixed decision policies . . . . . . . . . . 46

4.1.1 Decision rules based on constant or observed quantity 46

4.1.2 Decision rules based on quantity found using Bayesian updating .................... 51

4.2 Influence diagrams, LIMID _ . . . . . . . . . . . . . . 52

4.3 Markov decision processes . . . . . . . . . . . . . . 54

4.4 System effects . . . . . . . . . . . . . . . . 56

4.4.1 Correlations between variables . . . . . . . . . . 57

4.4.2 Influence of number of inspections/repairs on costs . . 57

5 Developing models for real decision problems 59

5.1 Learning of models using Bayesian networks . . . . . . . . . 60

5.1.1 Estimation of model parameters from data . . . . . . 60

5.1 .2 Estimation of model from data . . . . . . . . . . . . . 61

5.1.3 Estimation of causal relationships . . . . . . . . . 62

5.2 Case study: pitch gear deterioration _ . . . . . . . . . 62

6 Conclusions and outlook $\quad 65$

6.1 Outlook . . . . . . . . . . . . . . . 67

$\begin{array}{ll}\text { References } & 69\end{array}$

$\begin{array}{ll}\text { A Papers } & \mathbf{7 7}\end{array}$

A.1 Paper $1 \ldots \ldots \ldots \ldots \ldots \ldots$

A.2 Paper $2 \ldots \ldots \ldots \ldots \ldots$

A.3 Paper 3 . . . . . . . . . . . . . . . . . . . . . . . . . 103

A.4 Paper $4 \ldots \ldots \ldots \ldots \ldots$

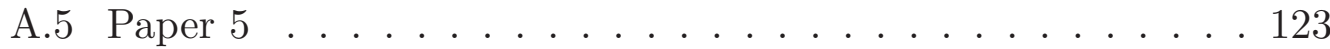

A.6 Paper $6 \ldots \ldots \ldots \ldots \ldots \ldots$ 


\section{Chapter 1}

\section{Introduction}

Renewable energy resources are becoming increasingly more important to reduce the greenhouse gas emission and to ensure the future energy supply. In Marts 2007, the countries in the European Union agreed on an overall target for a renewable energy share of $20 \%$ of the energy demand by 2020 (European Union 2009). Each country has a different share, and for Denmark the target is $30 \%$.

In 2010, Denmark covered 20\% of the energy demand with renewables, and in 2011 the Danish government came up with an energy proposal that should ensure 35\% renewable energy in 2020 (Regeringen 2011). Wind turbines play an important role in this, and they should cover $50 \%$ of the electricity demand in 2020, compared to 28\% in 2011 (Energistyrelsen 2012). A broad Energy agreement was settled in 2012, and the agreement contained offshore wind turbines of $1500 \mathrm{MW}$ before 2020 and an increase of the land based fleet of $500 \mathrm{MW}$ (Energiaftale 2012).

\subsection{Cost of wind energy}

The huge focus on offshore energy is primarily due to the not in my backyard mentality. Most people agree that wind turbines are a good solution for renewable energy, but they do not want to live close to them. Therefore, the number of good locations for onshore wind turbines are limited, and it is necessary to move them offshore. Other incitaments for building wind turbines offshore are higher mean wind speeds and less turbulence, which would lead to respectively larger energy production and less fatigue. However, offshore wind turbines are usually located in wind farms, where wake effects significantly increase the turbulence and reduce the energy production.

Looking at the bottom line, the total cost of energy is much larger offshore than onshore. The cost of energy varies between wind farms, but according to Engels, Obdam, and Savenije (2009) the cost of onshore wind energy is around $0.04 € / \mathrm{kWh}$, whereas offshore energy is twice as expensive, 
$0.08 € / \mathrm{kWh}$. One important reason is the large costs due to operation and maintenance (O\&M), which is twice as expensive offshore compared to onshore. The operation and maintenance costs contribute with around $25 \%$ of the cost of energy. The cost of energy can be calculated by (Engels, Obdam, and Savenije 2009):

$$
\text { cost of energy }=\frac{\text { turnkey costs }+ \text { capital costs }+ \text { O\&M costs }}{\text { energy production }}
$$

\subsubsection{Costs of O\&M}

The costs of O\&M can be divided into three categories: operations, planned maintenance, and unplanned maintenance. Operations cover day-to-day work necessary to keep the turbines running smoothly such as monitoring turbine operation, see Walford (2006). The planned maintenance includes greasing of bearings, changing oil, filters, pads and other smaller items, and control of various alignments and functions. These tasks are performed at intervals of varying length, starting from six months for the most basic service visits, and are usually defined in maintenance manuals by the turbine manufacturer.

The last part, the unplanned maintenance, makes up 2/3 of the direct O\&M costs (Engels, Obdam, and Savenije 2009). Unplanned maintenance is usually necessary when failure occurs, even in small components. The direct corrective maintenance costs go to spare parts, technicians, and vessels. For failures of larger components, larger vessels such as crane ships or jack-up vessels can be necessary, and these are very expensive. Failures generally enforce the turbines to shut down until they are repaired, resulting in additional indirect costs due to lost production. This amount is increased for wind farms located far from the shore in harsh environment, as relatively calm weather is required for the turbines to be accessed and repaired, and a substantial waiting time for an appropriate weather window can occur.

To reduce these costs, several parameters can be optimized. The maintainability can be increased by making it easier to maintain the components, for example, making it possible to exchange some subcomponents, without changing the entire component. This can make repairs cheaper and faster, and reduce the size of vessel needed. Thereby, the amount of lost production is reduced. Secondly, the accessability can be increased by using more sophisticated vessels.

Increasing the reliability will decrease the number of failures. This can be done by making a more reliable (expensive) design, using better design methods, using redundancy on critical components, or use preventive maintenance. Preventive maintenance is performed when a component is believed to be damaged, but before an actual failure happens. 


\subsubsection{Failure statistics}

Within the area of structural reliability, the event of failure is often a catastrophical event with huge consequences and, therefore, a low probability of failure is required (annually around $10^{-6}$ ). However, for wind turbines, a total annual failure rate between 1.5 and 4 can be observed, see Engels, Obdam, and Savenije (2009). Of course, only very few of these failures are actual collapses of tower or blades. A failure is in that context defined as an event where the wind turbine shuts down and needs interaction from humans to be started again.

Figure 1.1 shows mean failure rates and downtime per failure for German onshore wind turbines in the WMEP program (Faulstich, Hahn, and Tavner 2011). The downtime is an almost direct measure of lost production, and is also an indirect measure of the total consequences, as more expensive repairs generally imply longer downtime. The failures are divided into subsystems and into minor and major failures, where minor failures have a downtime of less than one day. In total, there are 1.8 minor failures per year and 0.6 major failures. The downtime for major failures in the electrical system is low compared to other major failures, but the failure frequency is large, and therefore the contribution to the total annual downtime is large. Major failures in larger mechanical and structural components such as the gearbox, generator, support, and drive train are rare, but when they happen, they come with a substantial downtime.

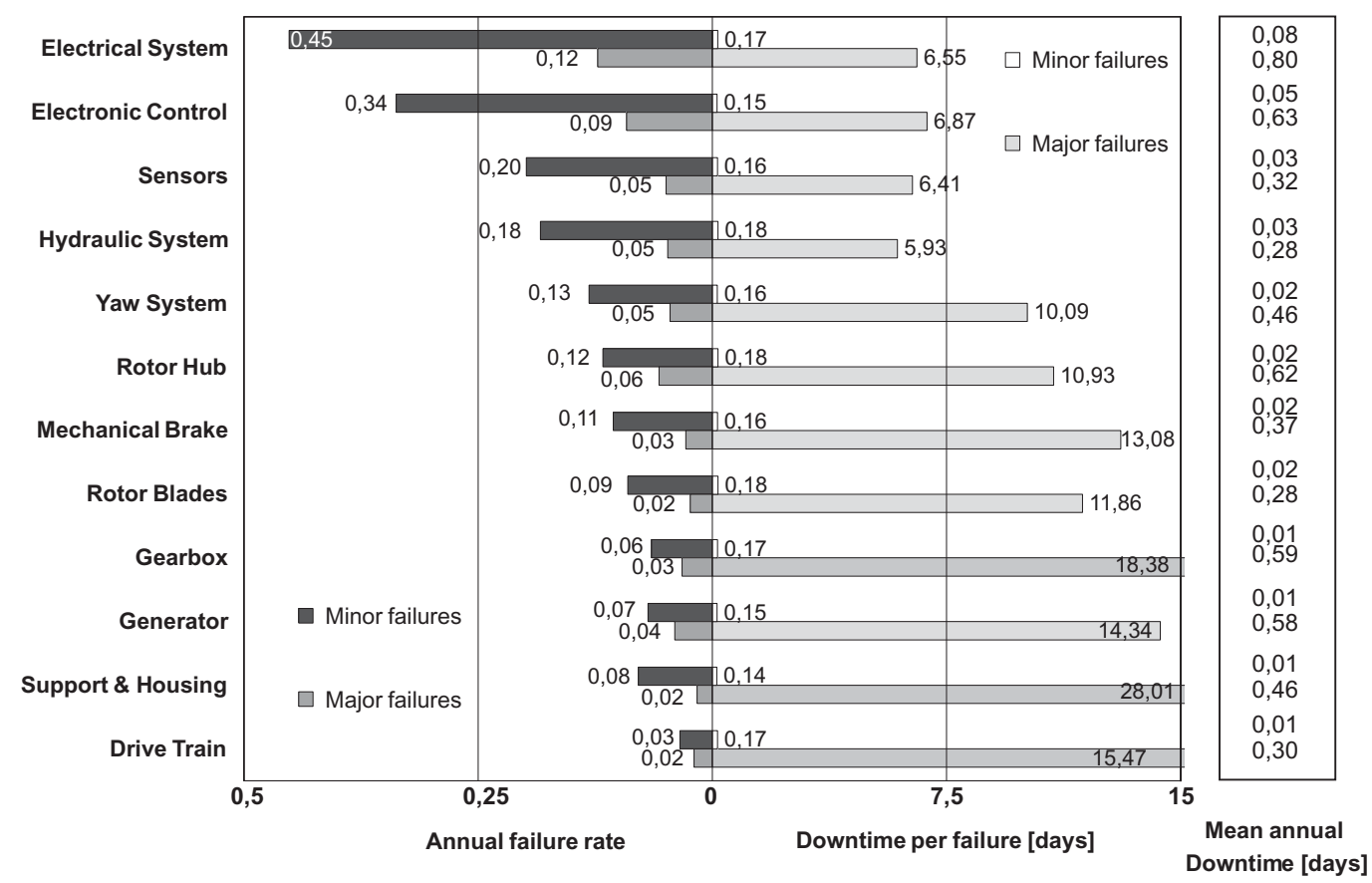

Figure 1.1: Failure statistics (Faulstich, Hahn, and Tavner 2011). 
The downtime illustrated above is for onshore wind turbines. For offshore wind turbines, the downtime is generally much larger, because of the limited accessability (Faulstich, Hahn, and Tavner 2011).

The downtime can also be expressed through the availability, defined as the proportion of the time a turbine is operational. For onshore turbines, the availability is typically high, above $98 \%$, but for offshore wind turbines availabilities in the range of $75-93 \%$ have been observed (Engels, Obdam, and Savenije 2009).

\subsubsection{Failure types}

The distribution of failures during the lifetime can often be represented using the bathtub curve, as shown in figure 1.2. The bathtub shape arises when three curves are added: the infant mortality curve, the constant failure rate curve, and the wear out curve.

The infant mortality curve includes failures that happen due to design errors, poor adjustments, and manufacturing errors, and is characterized by failures due to problems that are later solved and consequently not seen again during the lifetime.

The wear out curve arises from failures due to deterioration. As the lifetime of the wind turbine is going towards the end, so does the design lifetime of some of the components due to damage accumulation. Due to uncertainties in the design, some components start to fail. For deterioration processes, preventive maintenance can generally be used to prevent these failures.

The constant failure rate curve includes failures that are distributed evenly throughout the lifetime. Sometimes these failures are referred to as random failures, because they often seem to follow a poisson process with constant failure rate, which characterizes random failures. For random failures, the components transfer directly from the healthy state to the failed state, and it is not possible to forecast when it will happen. Moreover, preventive maintenance does not decrease the probability of failure, and is therefore of no use. However, the constant failure rate pattern can also be caused by deteriorating components with a relatively short life time. After the first years, stationary conditions set in, and so these wear out failures occur with a constant rate. For this type of failures, preventive maintenance can indeed be used.

\subsection{Maintenance planning}

As explained in the previous section, preventive maintenance can be used for deteriorating components, where the components do not go directly from the healthy state to the failed state, but instead there should be a damaged intermediate state. For these components, it makes sense to do preventive 


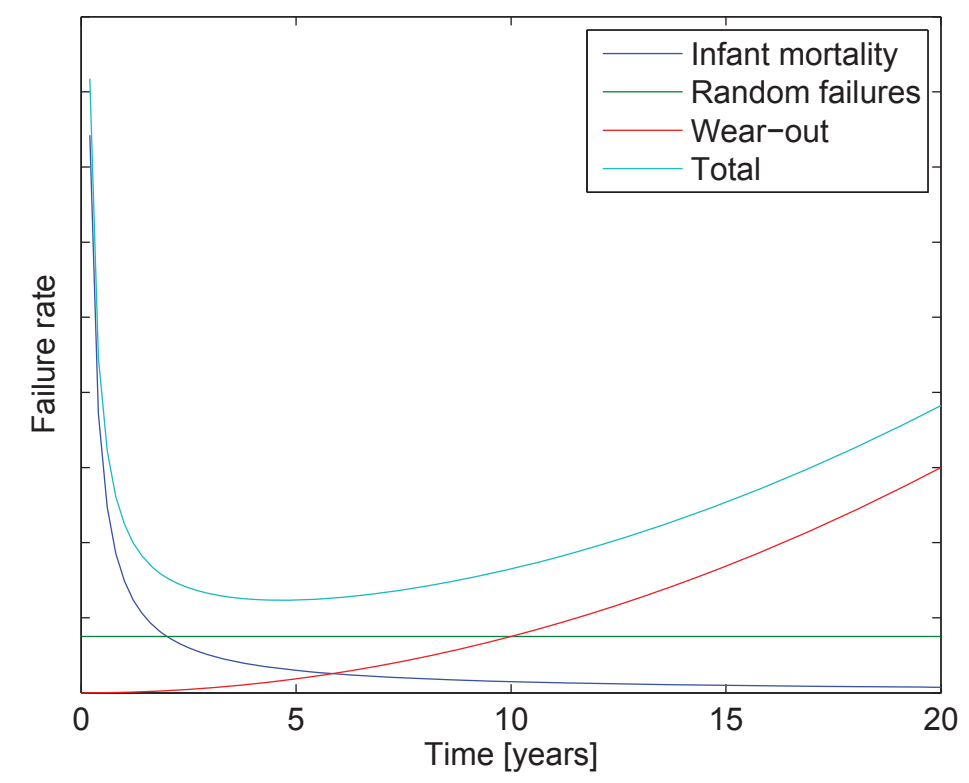

Figure 1.2: Bathtub curve.

maintenance, but it will not necessarily lead to lower costs if the maintenance effort is not balanced. The concept is shown in figure 1.3. For a higher maintenance effort, the failure costs are reduced, but the preventive maintenance costs are increased. The optimal amount of maintenance is the amount which leads to the lowest total costs.

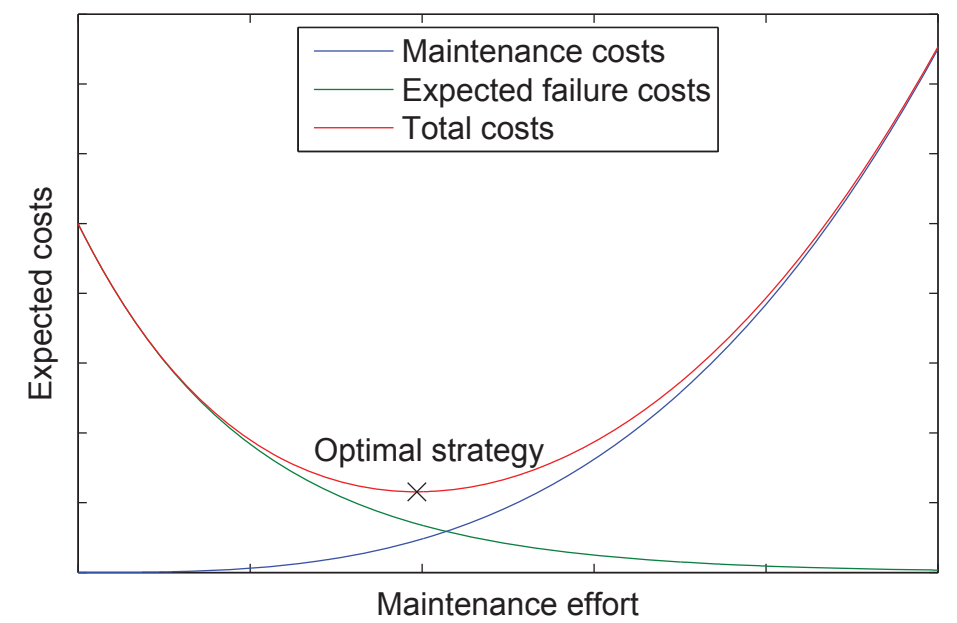

Figure 1.3: Optimal maintenance effort. 


\subsubsection{Preventive maintenance}

Generally, there are two methods for preventive maintenance, scheduled and condition-based, which are illustrated in figure 1.4.

For scheduled preventive maintenance, maintenance actions are scheduled already in the planning phase, and the method can be used if the lifetime of the component is known with large confidence. In case of larger uncertainty in the deterioration models, scheduled preventive maintenance is generally not worth the effort (Dekker and Scarf 1998). Instead, conditionbased maintenance can be used, where the maintenance decisions are made based on information about the actual health of the component, which can be obtained by use of for example condition monitoring.

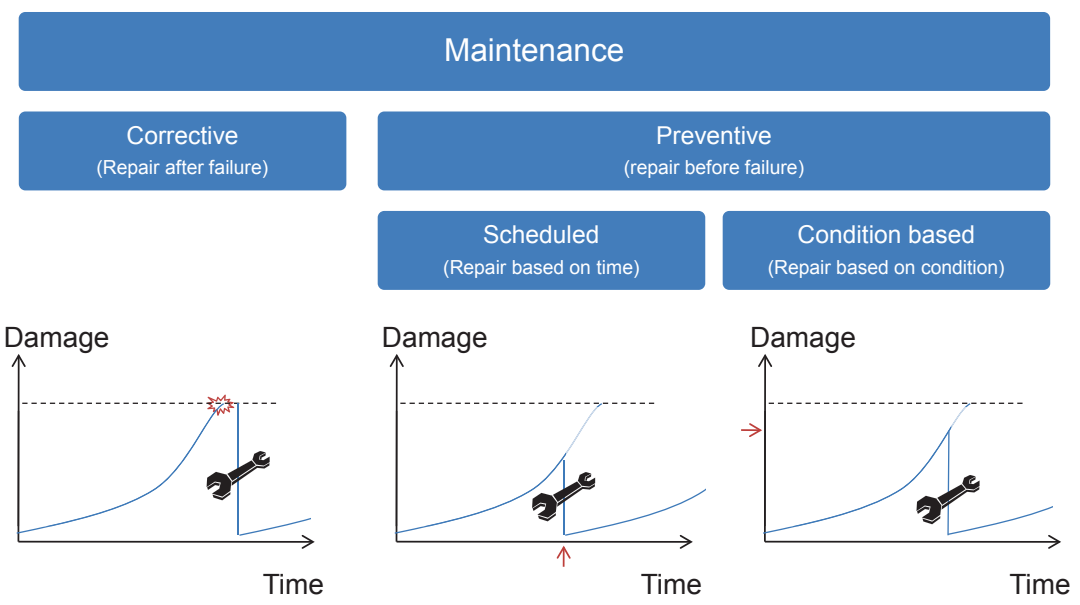

Figure 1.4: Maintenance strategies.

\subsubsection{Condition monitoring}

Various kinds of observations can be used for condition-based maintenance, either obtained using an online monitoring system, or by doing offline inspections. Offline observations can for example be visual inspections, oil sample analyses, and online measurements can be either condition monitoring or load monitoring. See Hameed et al. (2009) for a review of various methods for condition monitoring.

According to Wiggelinkhuizen et al. (2008), online monitoring should be used if the time interval between inspections is too large to detect damages before failure. But often the inspection methods are different, and online monitoring is also capable of detecting where the damage is thus pointing out where to inspect.

Generally, these observations are not perfect measurements of the size of the damage because the measurements are subject to uncertainty. Instead, they are indicators of the size of the damage, and the uncertainties on these 
indicators should be taken into account. The indicators can be used to provide a better estimation on the health of the component, and maintenance decisions can be made based on this estimation.

\subsubsection{Maintenance decisions}

The main decisions concerning maintenance of offshore wind turbines are decisions on when and how to obtain information, and when and how to do repairs. The decisions on which systems to use are normally made at the start of the lifetime, even though it can also be installed later. The decisions on offline inspections can be made continuously during the lifetime. The information obtained from monitoring and inspections is then available for the decisions on preventive repairs, which can also be made continuously during the lifetime.

For these decisions, not only the believed damage state is important, but also the weather forecast and season. In the winter, it might not be possible to perform a given repair, and therefore there are operational decisions on whether the turbine should be stopped until the repair or maybe run with lower loads. In addition to these main decisions, there are decisions on which vessels to use, which parts to have in stock, and how certain the weather forecast should be before a repair is initiated.

The decision problem contains decisions for all components, for all turbines in a wind farm; or even for several wind farms. And each of the components can be divided into subcomponents, where each of them will have different failure modes. This sums up to a large number of failure modes, which again has different costs for repairs, different vessel and crew requirements, duration, and failure rates/mechanism. These can be considered separately, but often the lower costs can be obtained by considering system effects. For example, if a repair is scheduled where a vessel with high mobilization costs is needed, more repairs can be made at the same time at a lower cost.

\subsubsection{Costs and consequences}

When decisions are made, the costs should be taken into account. During the lifetime, the costs go to:

- Inspections

- Preventive maintenance

- Corrective maintenance

These can include direct costs to crew, vessels, spare parts and indirect costs to lost production. Lost production is especially a contributor for corrective maintenance, as turbines are usually stopped in the period between failure 
and corrective repair. For inspections and preventive repairs, the turbine is only stopped while the operation is being performed, and it can be scheduled in periods with low wind. The costs of maintenance actions can vary significantly depending on whether it is a major overhaul or just a minor repair.

The length of the period between failure and corrective repair highly depends on the weather at the location and the availability of crew, vessel, and spare parts needed for the repair. Operators can reduce this mobilization time by having spare parts in stock and buying their own vessels instead of renting. Usually smaller vessels for smaller operations will be owned by the operator, whereas larger vessels will be rented. For larger repairs, the type of repair sets requirements which determine which vessels to use. But often, several options are available, and for a higher prize a vessel with less strict weather limits can be used. This leads to lower costs to lost production and higher direct costs for repairs. Generally, the longer the duration of the repair and the more strict weather requirements, the longer is the expected waiting time for an appropriate weather window. Some repairs can be split up into parts and probably will be if the repair duration and transport forth and back is longer than the work day.

The general reason for making preventive repairs is to reduce costs to corrective repairs. In addition to the reduced downtime, the repair can be cheaper to make before failure. Also, if a subcomponent fails, there is a chance that the failure will cause other subcomponents to fail. For example, a damaged gear tooth in a gearbox can be preventively repaired by changing that gearwheel. But if the gear tooth fails and breaks off, the loose tooth can cause the entire gearbox to fail, such that a much more expensive repair is needed.

On the other hand, preventive repairs can also cause damage to other components. When a gear bearing is changed, the shaft is heated and the bearing hammered off. This induces both thermal and impulse loads, and can initiate damages on other shafts. Likewise, during a repair that requires dismantling of the rotor or a rotor blade, there is a risk that the blade is damaged. The costs of these types of consequence damage should in principle also be taken into account.

\subsubsection{Current maintenance practice}

Generally, wind turbines are equipped with SCADA (supervisory control and data acquisition) systems, which monitor a long list of parameters such as wind speed, productions, tower vibrations, etc. Often there are more than a hundred parameters, for which the 10-minute statistics are logged.

The control system in wind turbines automatically sets off an alarm, if it detects that anything is wrong, and some alarms cause the turbine to stop producing power. Sometimes, a remote restart is sufficient to get the turbine 
up running again, and in other cases, it is necessary to send technicians to the turbine to make a repair. In case of more complicated malfunctions, one or more inspections are needed to figure out exactly what the problem is, and then a corrective repair can be scheduled.

In modern multi-megawatt turbines, it is normal to have a condition monitoring system installed. The system measures among others vibrations at a number of locations in the drivetrain, for example at the generator, main bearing, and several places at the gearbox. It can detect damages by increased vibrations on specific frequencies. Furthermore, as each type of damage has a specific frequency, it can also detect where a damage is, which makes it easier for the technicians to locate a damage. Alarm levels are set for the vibration measurements, and when the operator is noticed of an alarm, they need to decide what to do. Usually, an inspection is made to investigate whether it is a false alarm or how servere the damage is. If a damage is present, it needs to be decided when to make a repair. Should it be done immediately, or can they for example wait several months until the summer? For this assessment, a prognosis for the damage progression is necessary. Usually, this prognosis is made based on operational experience, but without using any advanced models or optimization techniques for decision support.

\subsection{State-of-the-art for planning of maintenance for offshore wind turbines}

In this section, an overview is given of the state-of-the-art in the literature for optimal maintenance planning for offshore wind turbines. Most methods are variants of risk-based methods which means that consequences and uncertainties are included in the analysis. The analysis can be qualitative or quantitative, and almost all quantitative methods aim at doing cost optimal maintenance planning by minimizing the life cycle costs, usually defined as the expected costs.

Some of the methods focus on estimating costs to corrective operation and maintenance, and can be used for decision making for e.g. vessel types, whereas other methods are used for preventive maintenance planning. To assess the costs of preventive maintenance, one of the most important things to consider is the relationship between reliability and maintenance. In other words: how does monitoring, inspections, and repairs affect the reliability.

\subsubsection{Estimation of costs to corrective maintenance}

The methods and models considered in this section, are models for estimating the costs to corrective maintenance. For theses methods, the failure rate is a sufficient description of when failure occurs. The methods are either 
based on annual mean values or Monte Carlo simulation.

\section{Mean value based}

The O\&M tool is an Excel based program developed by the ECN (Rademakers et al. 2003) and has been certified by Germanischer Lloyd (Rademakers et al. 2008b). The overall expected costs to corrective maintenance are estimated based on failure rates and expected failure costs for each failure type. The expected failure costs also include the costs due to lost production. To estimate these costs, expected waiting times for weather windows of given lengths are needed for each vessel type. Those are found based on the processing of time series of significant wave heights and mean wind speeds. The mean wait time is found for a number of weather window lengths, and a polynomial is fitted to describe the mean waiting time as function of the length of the needed weather window. These curves are used to calculate the lost production associated with each failure type. As input, the program needs the overall failure rate for each component, division into failure types with matching maintenance classes with specified duration, weather limits and costs.

The O\&M tool can be used to assess the influence of using different vessels or using more reliable or maintainable components. However, it is not possible to split up large maintenance actions into more weather windows, and this gives an overestimation of the waiting time for repairs with long duration (Rademakers et al. 2008b). Also, it cannot be assessed how many vessels and crews are needed, as the program is based on mean values and does not take system effects into account.

The strength of the program is the modeling of expected waiting time and the way the failure types are structured.

\section{Simulation based}

Several simulation based tools have been developed for assessing the costs of corrective maintenance:

- CONTOFAX from Delft University of Technology (van Bussel and Bierbooms 2003)

- O2M from GarradHassan (Philips, Morgan, and Jacquemin 2006)

- MWCOST based on SLOOP from BMT (Stratford 2007)

- OMCE from ECN (Rademakers et al. 2008a)

Furthermore, a model called NOWIcob is currently being developed by NOWITECH (Hofmann et al. 2011). MWCOST from BMT is developed in the simulation tool SLOOP, which have been used for maintenance planning 
in the oil and gas industry. OMCE is an abbreviation for Operation and Maintenance Cost Estimator and was developed in Matlab at ECN who also developed the O\&M Tool. Where the O\&M Tool can only be used to predict long-time average costs, the OMCE focus on short-time (1-5 years) decisions on number of vessels, crew, and spare parts.

The tools generally allows modelling of a wind farm with various failure types for each wind turbine. Generally, the input for the models consists of description of failure behavior, access and hoisting systems, and weather conditions. Monte Carlo simulations are run in the time domain, and failures are simulated based on the failure rates. Each failure type belongs to a certain maintenance category, which determines the weather limitations and vessel, crew, and time needed for the repair. The repair will be performed as soon as the weather allows for it, and the turbine will not produce power until the repair is finished. The models also keep track of vessels, crews, and spare parts, such that it is necessary to wait until vessels are vacant before using them for another repair. Thus, the influence the number of available vessels and crews has on the availability and the costs can be assessed. If spare parts are in stock, the logistical time before the repair can be made is smaller than if they have to be ordered after the failure happens.

The models are similar in many aspects, but there are also some differences. Failures can be simulated from a Poisson process with constant failure rate in all models, which corresponds to random failures. However, MWCOST also includes an option of time dependant failure rates. The models generally include scheduled maintenance and the effect of it on the availability.

There are some differences with regards to the weather modeling. For the OMCE and MWCOST, time series are used as input to the model and used directly in the simulations. For MWCOST, it is recommended to use hindcast data. The O2M model generates pseudo random time series of the significant wave height using Fast Fourier Transformation (FFT) based on the input time series, and inverse FFT to create artificial time series. Phases corresponding to tidal and seasonal variation are fixed, and the other phases are random. This method can indeed be used for high frequency time series of surface elevation, but here it is applied to low frequency time series of significant wave height. Thus this process will generally result in some of the significant wave heights being negative, and this issue is not addressed in (Philips, Morgan, and Jacquemin 2006). In CONTOFAX, the input is time series of accessible and inaccessible periods for each equipment type. These are generated based on Weibull distributions for accessible and inaccessible weather windows, which are found based on real time series.

The weather model also affects the results from the model in terms of availability and lost production. In CONTOFAX, only the availability is calculated, because the wind speed is not included in the model, and thereby the amount of lost production cannot be calculated. In the other three 
models, the calculation of lost production is based on the wind speed, and thereby the correlation between low accessibility and high lost production is captured.

The models differ in how accurate the repair is modeled. For O2M, the repair is started as soon as the weather is below the limits for repair, and it is paused if the limits are exceeded, thus no weather forecast is included. In CONTOFAX and OMCE, it can be specified how each type of maintenance actions can be split up. Here, the weather forecast is assumed to be $100 \%$ accurate. In MWCOST, a weather forecast including uncertainties can be included.

The part of OMCE described above is the OMCE Calculator. The OMCE also consists of building blocks for condition based maintenance, but these act as separate programs which give input to the OMCE Calculator about expected maintenance actions in the following period. One of the building blocks being developed is "Loads \& Lifetime" based on the Flight leader concept, (Obdam, Rademakers, and Braam 2009) (Obdam, Rademakers, and Braam 2010). Based on strain gauge load measurements from the blade and tower of two turbines in a wind farm, neural networks are used to estimate loads on other wind turbines in the wind farm based on SCADA data. It gives relatively good results for the studied cases, and the load accumulation is estimated based on the data. Another building block is called "Health Monitoring", and the CONMOW project was performed in connection with the development of that part (Wiggelinkhuizen et al. 2007) (Wiggelinkhuizen et al. 2008). Several turbines were equipped with condition monitoring systems, and data from those was analyzed together with the SCADA data. Generally, no failures happened in the measurement period, and thus it could not be shown to work. They conclude that necessary knowledge is not available to set alarm levels or to estimate remaining life time.

In addition to the tools described above, simulation based procedures have also been reported in (El-Thalji and Liyanage 2011) based on dynamic simulation.

\subsubsection{Decisions on preventive maintenance}

This section concerns models for decision making for preventive maintenance. The most simple decision is the decision on when to make a repair based on the predicted lifetime only, thus no inspections or condition monitoring is included. This type of model is used in (Bharadwaj, Speck, and Ablitt 2007) to make decisions for offshore wind farms, and a survey of maintenance policies for deteriorating systems is presented in (Wang 2002).

In this section, methods used in the literature for decisions for preventive maintenance using inspections or condition monitoring will be described. 


\section{Delay time maintenance model}

Andrawus et al. (2008) used the delay time maintenance model for estimating optimal inspection intervals for components in wind turbines. For the component health, three states are considered in the model:

- No detectable defect

- Detectable defect

- Failure

The delay time is the time between a defect becomes detectable and failure occurs. In this period, defects are repaired preventively if an inspection is performed. The optimal inspection interval can be found given the rate of arising defects, delay time distribution, and costs and durations for inspections, failures, and preventive repairs. Optimization can then be performed to reduce total costs or downtime.

One of the critical points in the method is to find the delay time distribution. It is possible to estimate it subjectively or objectively based on operational data. If inspections are already performed at a fixed interval and the number and time for failures and preventive repairs are known, the delay time distribution can be estimated objectively using a Maximum-Likelihood estimate of the parameters for the distribution. It can be estimated based on subjective data from the inspection crews finding defects, by summing up the estimated time since damage could first be detected and estimated time to failure. A distribution can then be fitted to the estimated delay times.

In Andrawus et al. (2008), the delay time distribution was estimated using the objective method, but based on subjective estimated input data concerning time from last inspection to failure. Out of seven considered subcomponents, optimal inspection intervals could be found for two of them. For the remaining it was not cost-efficient to make scheduled inspections.

The model used in Andrawus et al. (2008) is the basic model with randomly occurring damages, perfect inspections, and constant inspection interval. Some of the limitations in the basic case are possible to relax (Christer 1999) (Christer and Waller 1984):

- The rate of arising defects is constant. Relaxed: Can depend on time from new.

- Perfect inspections. Relaxed: A probability of detection can be included.

- Constant inspection intervals. Relaxed: Inspection intervals can be set based on reliability. 
One advantage of the method is that it is not computationally demanding. Another is that the concept of delay time is easy to understand for practitioners. The main limitation of the method is that it only concerns offline inspections/monitoring. Thus, it does not allow taking online monitoring measurements into account when the decisions on inspections are made.

In Andrawus et al. (2008), the inspections were assumed to be performed using vibration measurements. Modern offshore wind turbines are normally equipped with online condition monitoring systems with vibration measurements, and therefore it is not relevant to use this method to estimate the intervals for those measurements for those turbines.

\section{Reliability Centered Maintenance}

Reliability Centered Maintenance (RCM) have been used for maintenance planning for offshore wind turbines by Andrawus et al. (2006) and Nilsson and Bertling (2007). RCM has also been applied for maintenance planning in other industries, for example for bridges (Linneberg, Zinck, and Christensen 2012) (Bloomstine 2012). RCM is a systematic qualitative procedure that determines what to do to preserve system function, and it prioritizes critical components for system reliability. It was developed in the American airline industry and described first by Nowland and Heap (1978). A standard called SAE JA1011 was published by Society of Automotive Engineers (1999) and describes the basis of the RCM process as:

Any RCM process shall ensure that all of the following seven questions are answered satisfactorily and are answered in the sequence shown below:

1. What are the functions and associated desired standards of performance of the asset in its present operating context (functions)?

2. In what way can it fail to fulfill its functions (functional failures)?

3. What causes each functional failure (failure modes)?

4. What happens when each failure occurs (failure effects)?

5. In what way does each failure matter (failure consequences)?

6. What should be done to predict or prevent each failure (proactive tasks and task intervals)?

7. What should be done if a suitable proactive task cannot be found (default actions)?

The standard was needed, because the term RCM was being widely used, and it was necessary to define what a process must fulfill to be called RCM 
(Moubray 2000). Steps 1-4 in the analysis also correspond to the Failure Modes and Effect Analysis (FMEA), and steps 1-5 to the Failure Modes, Effects and Criticality Analysis (FMECA).

$\mathrm{RCM}$ is a structured qualitative approach for maintenance planning, and thus no optimization is performed. However, RCM is often combined with a qualitative optimization, and Dekker and Scarf (1998) argues that there is certainly a need for optimization.

Bertling, Allan, and Eriksson (2005) and Bertling (2002) combine RCM and asset maintenance method to reliability-centered asset maintenance (RCAM), and use it for maintenance planning for power distribution systems. In the model, the relationship between reliability and maintenance is modeled using both a constant relationship and a time dependent relationship.

Nilsson and Bertling (2007) used the RCAM approach for a life cycle cost analysis for wind power systems including condition monitoring. It was calculated, how much the need for corrective maintenance should be decreased to justify installing a condition monitoring system. Thus the relationship between maintenance and reliability was not modeled.

Also Andrawus et al. (2006) used a hybrid of RCM and asset life-cycle analysis for comparing the costs of time based and condition based maintenance. However, the costs for both methods are assumed without including how efficient preventive maintenance is in avoiding failures.

\section{Other preventive models}

This section gives an overview of other methods described in the literature, from which most are based on simulation.

In McMillan and Ault (2007) and McMillan and Ault (2008), the benefit of condition monitoring is quantified using a Markov chain solved using Monte Carlo simulations for onshore and offshore wind turbines respectively. A weather model is included in the simulations and thereby limited assess in modeled, and the amount of lost production is quantified. The model includes four critical components, but does not model system effects with respect to repairs, even though the decisions are made simultaneously. The costs of preventive repairs are assumed to be $10 \%$ of the component cost. For corrective maintenance, repairs are assumed to be possible for $50 \%$ of the failures, for the remaining $50 \%$ a new component is needed. Condition monitoring is initially assumed to be without uncertainties, and the optimization is made with respect to the wait time from the component enters the deteriorated state until a preventive repair should be made. A sensitivity study is made to assess the influence of the success rate of the condition monitoring system, but false alarms are not taken into account, and the succes rate does not increase with increasing damage size, as only one intermediate damage state is included in the model. 
Andrawus, Watson, and Kishk (2007) used a reliability block diagram to optimize wind turbine maintenance, and called the approach Modelling System Failures, see also Andrawus (2008). Failure data is fitted to the Weibull distribution, and the failure type is determined based on value of the shape parameter to be infant mortality, random, or wear-out. In case of random failures, condition based maintenance is argued to be most suitable, and for components with wear-out failure pattern, the optimal replacement time is found in a separate analysis. The total costs are assessed, but the influence of condition based maintenance on failures is not modeled.

Besnard, Nilsson, and Bertling (2010) calculates the benefit of using condition monitoring using simulation. Failures are simulated from a Weibull distribution, and a constant succes rate is assumed for the condition monitoring and for reduction of consequential damage. The downtime after failure is assumed to be constant.

In Besnard and Bertling (2010), the optimal strategy is found for maintenance for wind turbine blades. Two inspection types and online monitoring are compared, and for inspections the optimal inspection intervals are found. The expected costs are found using simulation, and the deterioration is described by a Markov model with five states. The uncertainties for visual inspections and condition monitoring are taken into account and depend on the deterioration state, but no false positives occur.

Tian et al. (2011) presented a simulation based optimization for condition based maintenance. A constant inspection interval was assumed, and the optimization parameter was the threshold for the probability of failure in the lead time. If the threshold was exceeded, a preventive repair was made. The lead time is the time interval until the preventive maintenance could be performed, and therefore it does not give physical sense to use this probability of failure, as it is not reduced by the preventive maintenance action. The prediction of lifetime distribution was assumed to be based on neural networks with monitoring measurements and age as input and life percentage passed by as output. The training procedure gives the standard deviation for the normal distributed error. At the time of each inspection, the probability of failure is estimated from the same lifetime distribution, which is a normal distribution around the true time of failure, thus the prediction does not change with time. In reality, new information is obtained each time an inspection is made, and the distribution should be updated in a consistent manner.

Besnard et al. (2009) and Kovacs et al. (2011) both focus on short term scheduling of maintenance actions to reduce lost production by using periods with low wind. The scheduling problems are solved by linear integer optimization models, and knowledge of the weather is assumed to be available from a forecast. Maes and Naess (2012) concerns the general problem of selecting an optimal window of opportunity within a finite horizon, and investigates different strategies to choose the right window of opportunity. 
This could be the approach if knowledge of the weather is only available for a period corresponding to the current weather window.

Welte and Eggen (2008) use Bayesian methods to estimate sojourn time distribution parameters combining expert opinion and data.

\subsubsection{Conclusions on state-of-the-art}

Detailed simulation-based methods exist for determining the total costs to corrective maintenance. But for preventive maintenance, none of the methods reviewed above adequately take into account the relationship between maintenance and reliability, and they cannot be used directly for optimal maintenance planning taking into account monitoring, inspections, preventive repairs and corrective repairs. To do so, methods can be developed from a Bayesian perspective as described in JCSS (2008). A Bayesian framework have been used successfully for planning of O\&M for oil and gas structures under the name Risk-Based Inspection (RBI), and can potentially be applied to offshore wind turbines as well. The term Risk-Based Inspection (RBI) has been used for various methods, both qualitative and quantitative. American Petroleum Institute has published the API RP 580 which concerns Risk-Based Inspected for the oil and gas industry, with a qualitative approach (API 2002). The RBI procedure considered here is quantitative and based on Bayesian pre-posterior decision analysis as described in Faber (2002).

\subsection{Pre-posterior decision analysis}

When a decision problem contains more than one decision, and earlier decisions influence later decisions, the problem is called a pre-posterior decision problem, as introduced by Raiffa and Schlaifer (1961) (see also Benjamin and Cornell (1970)). For pre-posterior decision problems, optimal decisions are made such that the expected costs are minimized. When a decision is made, it is necessary to take into account the optimal decisions for all future decisions. Also, see paper 1 and Sørensen (2009) for a description of the pre-posterior decision analysis for maintenance for offshore wind turbines.

For the basic pre-posterior decision problem, the true state of world is unknown, and first a decision is made on whether to make an uncertain observation. This observation is then available for deciding whether a repair should be made. The total expected utility depends on the true state of nature, the observation, and the two decisions. For inspection and repair planning, the decision problem can be illustrated in a decision tree as shown in figure 1.5, where time is discretized.

There are in principle two methods for solving pre-posterior decision problems, extensive and normal form. In the extensive form, the starting point is the final branches, and the optimal decisions are first found for 


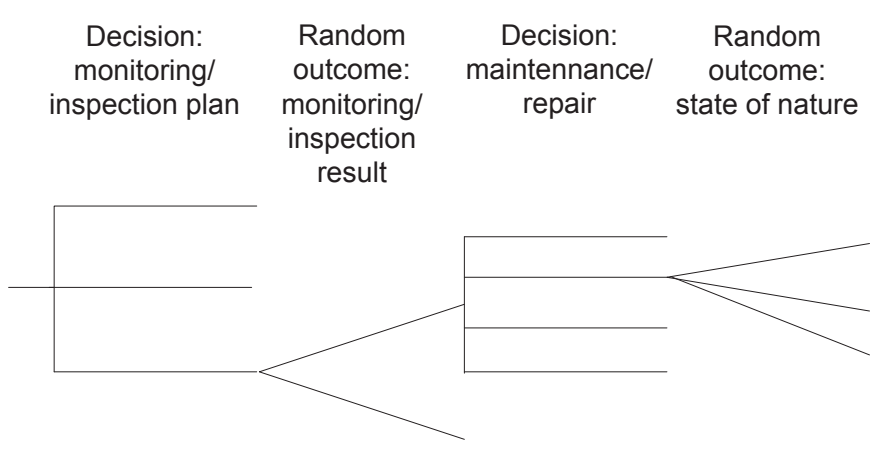

Figure 1.5: Decision tree for one time step. Repeated for each time step.

the last decision, then the second last, using the result from the previous branch, and so on. Thereby, a minimization is performed for all decisions in the decision tree. The number of branches, and thereby the computation time, increases exponentially with the number of decisions (e.g. time steps), and therefore the problem easily becomes intractable.

The normal form analysis uses decision rules for the decisions based on the outcome of past observations and decisions. For each decision rule, the expected utility is found by computing probabilities and utilities for all branches and taking the expected value. The decision rule with the largest expected utility is then chosen. This analysis form gives the same result as the extensive form analysis if every possible decision rule is considered. This means that the entire past should be included in the decision rules, and there should be a unique decision rule for each time step. Here, the computation time is also increasing exponentially with the number of time steps, and therefore approximations are generally necessary for pre-posterior decision problems with many decisions.

\subsubsection{Risk-Based Inspection}

The development of RBI started in the early 1970, and the development of structural reliability methods was an important step, (Madsen, Krenk, and Lind 1986), (Madsen, Tallin, and Kirkemo 1987), (Madsen, Sørensen, and Olesen 1989), (Madsen and Sørensen 1990). As described in the framework by Faber (2002), the methodology is based on the pre-posterior decision analysis with appropriate approximations. See also Straub (2004) for a detailed description. A typical decision tree is shown in figure 1.6. To evaluate the probabilities in the decision tree, a probabilistic damage model and inspection model are needed, and Bayes rule is used for updating the model. This is usually done using reliability methods such as FORM, SORM, or Monte Carlo methods.

The first approximation is the use of same decision rule for repairs at every time step and only taking into account the most recent inspection 


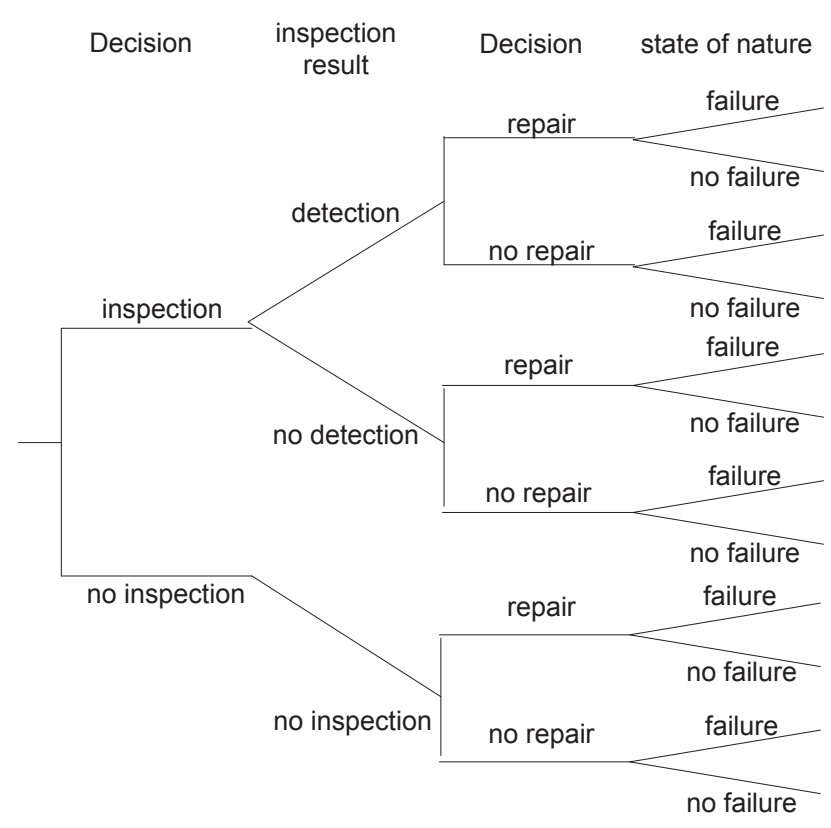

Figure 1.6: Typical decision tree for RBI for one time step. Repeated for each time step.

result. The simplest rule is repair at detection, but the rule could also be to repair damages exceeding a certain size. When a fixed repair strategy that only depends on the inspection outcome is used, each inspection outcome does not create a branch. Only different repair actions create a new branch to be evaluated, because the previous inspection outcomes are not taken into account in future decisions. Secondly, failure is assumed to be a terminal event, meaning that the lifetime ends after a failure. In case of preventive repairs, the component can be assumed to act like a new component or a component with the correct age but no detections.

With these assumptions, only two of the branches in figure 1.6 continue; no inspection and inspection followed by no detection. However, this still gives a computation time increasing exponentially with the number of time steps. To avoid this, inspections can be scheduled based only one parameter: time between inspections or probability of failure. For both inspection strategies, the optimal inspection plans are found when no detection and no failure are assumed. With these assumptions, it is possible to compute the total expected costs for different strategies for inspection and repairs, and find the strategy which minimizes the expected costs. In case of repairs, the inspection plan afterwards depends on the assumption for repairs. If they are assumed to act like components with no indications, the initial inspection plan is used. If they are assumed to act like new components, inspection plans corresponding to a lifetime equal to the remaining lifetime is used.

The approach described above considers RBI for one component or hot 
spot. Several papers address system effects, which can be taken into account in order to reduce the costs. In Straub and Faber (2004) and Straub and Faber (2005), the effect of inspecting one hot spot on the other hot spots were taken into account through the concept of Value Of Information from the Bayesian decision theory.

Development of optimal inspection plans are generally computationally demanding, because many Monte Carlo simulations are required to obtain an accurate estimate of the probability of failure. This issue was addressed in Faber et al. (2005), Straub and Faber (2006), and Straub (2004), where generic inspection plans were developed based on the Fatigue Design Factor (FDF) for welded connections.

\subsection{Objective of thesis}

Many failures in wind turbine components happen due to deteriorations processes such as fatigue, wear, and corrosion, for which preventive maintenance can be used. Preventive maintenance is used in the industry, but is not planned using methods which takes into account all available information from condition monitoring, inspections, load measurements, and theoretical models. This can potentially be done using Bayesian methods, which have been used in the oil and gas industry for RBI.

However, RBI is only computationally feasible when approximations are applied as described above. One of the assumptions used in RBI is to consider failures as being terminal events. This makes sense in the oil and gas industry, where the annual probability of failure is in the order of $10^{-5}$. However, for wind turbine components, the probability of failure is generally much larger, and that assumption cannot be justified. Furthermore, RBI does not take information from condition monitoring and load measurements into account, and therefore RBI cannot directly be applied to offshore wind turbines in the current form. This thesis concerns the development of such methods for offshore wind turbines, and the hypothesis for the research is the following:

Using basic theories from Bayesian statistical decision theory, a reliabilitybased basis for cost optimal planning of operation and maintenance for offshore wind turbines can be developed to assist in decision making on planning of $O \& M$, implying a substantial reduction of the total lifetime O\&M costs.

\subsubsection{Approach}

The approach contains three main parts:

- Development of probabilistic damage model 
- Updating of model estimate with observations using Bayesian updating

- Making risk-based decisions based on the updated model, taking into account future unknown observations

In this way, the prior knowledge from the design methods and past experience can be combined with observations, taking the uncertainty on each type of information into account. Cost optimal decisions on maintenance should then be made such that the expected life-cycle costs are minimized. The starting point is the Bayesian pre-posterior decision analysis, but the computational difficulties need to be overcome.

The three main parts of the approach are presented in the next three chapters in this thesis, and the following chapter concerns development of models for real decision problems. The last chapter contains conclusions and outlook. 



\section{Chapter 2}

\section{Deterioration modeling}

A probabilistic deterioration model is necessary for the approach for riskbased planning presented in this thesis. The model should be established using all information available at the time, for instance the theoretical model used in the design, data from past experience, and expert knowledge. The model can be continuous if the damage size can be quantified on a continuous scale, or be discrete if the health state can more appropriately be quantified on a ordinal scale. For deterioration processes, damages are generally increasing with time. Thus, the health state transfers to states with more servere damage with time.

In traditional reliability theory, the sojourn time in each state is often described by a probability distribution, as in the delay time maintenance model (Christer 1999). For a Poisson process, the exponential distribution is the sojourn time distribution. Alternatively, time can be discretized, and the probability of transferring to the next state is constant for each time step. Then, the problem is described by a Bernoulli process, and the sojourn time is described by the geometrical distribution. The latter approach will be used here due to its relation to the Markov process. The approach can also be used for a discretized continuous damage size. Here, it will usually be possible to skip a damage state, i.e. move two or more damage states in one time step.

Two requirements should be fulfilled for a damage model to be used for this approach. First, there should be at least three states if a discrete model is used. If there are only the two states healthy and failed, preventive maintenance does not affect the probability of failure. Secondly, it should be possible to make a link between the damage state and the probabilities of each condition monitoring outcome. In other words, the damage size should be measurable.

Generally, deterioration models can be developed based on design models or on data. The case where data is used is treated in chapter 5, whereas this chapter concerns the development of probabilistic deterioration models 
from theoretical models. The design models are often deterministic models, but they are subject to uncertainties of different types, and these uncertainties should be taken into account when the models are used for risk-based decision making.

The uncertainties can be divided into two categories: aleatory and epistemic. The aleatory uncertainties are physical uncertainties, and thus they are irreducible. The epistemic uncertainties are caused by inaccurate models and inadequate statistical data and are reducible. For strength variables, distinction should be made between the situations before and after the components are produced. Before they are produced, aleatory uncertainties are present due to the variability in the performance of components made in the same production process. This uncertainty cannot be reduced without changing the production process. Laboratory testing can be used to assess the variability arising from the production process, but this only reduces the epistemic uncertainties. Once the component has been produced, the aleatory uncertainties arising from variability in the production process become epistemic. Therefore, it is possible to reduce these uncertainties when a specific component is considered. They can be reduced by making observations of the degree of damage during the lifetime of the component. See Kiureghian and Ditlevsen (2009) for an extensive discussion on aleatory and epistemic uncertainties.

\subsection{Fatigue design models}

This section describes the general method used in fatigue design and the uncertainties associated with the method. For structural elements, fatigue usually happens in locations with high stress concentrations, such as welded details. But local stress concentrations are also present in the mechanical components in a wind turbine, and fatigue can be the design driver for many components. The three main types of components for fatigue in wind turbines are blades, cast components (and non-welded steel details), and welded details. Fatigue is characterized as the failure of components due to cyclic loading. It is a failure mechanism which is not fully understood, and in the design, therefore, the empirical SN model is usually used together with the Palmgreen-Miner linear damage accumulation law. See Stephens et al. (2001) for thorough description of fatigue, and Straub (2004) for a thorough description of deterioration modeling for RBI.

Fatigue failure can be divided into three stages: crack initiation, crack propagation, and brittle failure. Brittle failure happens when the crack becomes unstable. This division is a convention based on observations, as the underlying micro-mechanics are common. The fatigue life of a component is the sum of the crack initiation life and the crack propagation life. In the SN model, no distinction is made between initiation and propagation. In this 
section, fatigue design models based on SN curves are presented. In section 2.2 , the fracture mechanical approach where a measurable damage size is included is presented.

\subsubsection{SN curves}

The number of load cycles until failure depends on the amplitude/stress range of the cycles. For loading with larger stress ranges, failure happens at a lower number of cycles. SN curves are used to describe this relationship between the number of cycles to failure, $N_{F}$, and the stress range, $\Delta S$. They were first described by Wöehler in 1860 based on experiments with constant stress amplitude. The simplest case is described by the Basquin Equation, which predicts a linear relationship between $\log N_{F}$ and $\log \Delta S$ :

$$
N_{F}=C \cdot \Delta S^{-m}
$$

The constants $C$ and $m$ can be determined empirically using tests, and values for specific applications can be found in the design codes. The SN curves are usually specified as characteristic curves with $95 \%$ survival probability. The reliability of the SN curve can be determined if a large number of tests is made. Usually, the slope of the SN curve is not constant within the entire range of stress ranges, thus a multi-linear curve is often used, as shown in figure 2.1.

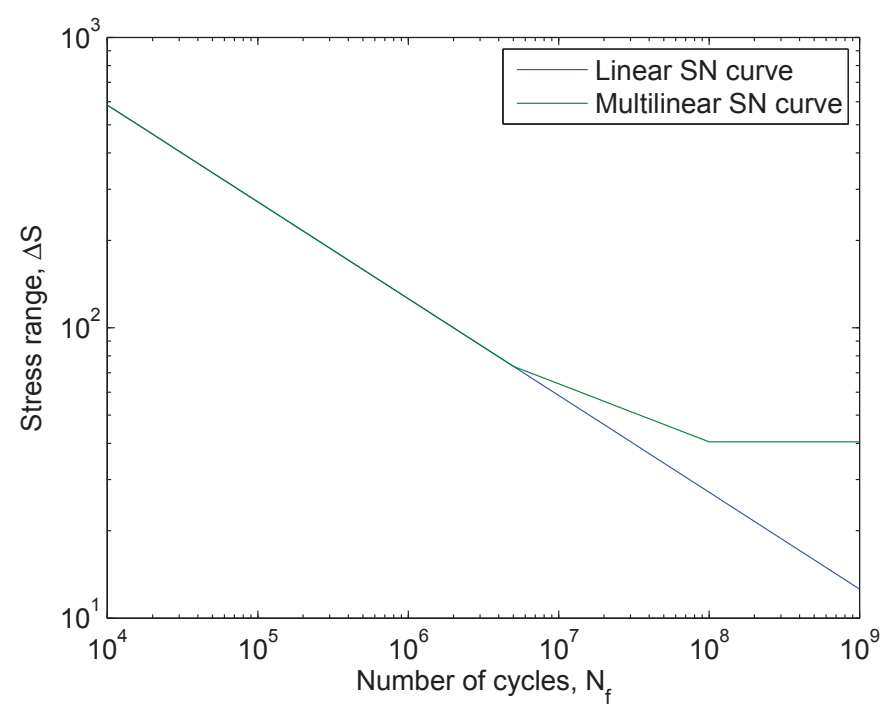

Figure 2.1: Multi-linear SN curve after Eurocode 3-1-9 (2005).

\subsubsection{Palmgreen-Miner damage accumulation law}

SN curves specify the number of cycles to failure at different constant stress ranges. Often, the stress ranges for a component vary in size, and the 
Palmgreen-Miner damage accumulation law is used to deal with this (Miner 1945). It is based on the assumption that the order in which the cycles occur does not influence the total damage. For each stress cycle, the damage increment, $\Delta D_{i}$, is calculated by:

$$
\Delta D_{i}=\frac{1}{N_{F, i}}
$$

$N_{F, i}$ is the number of cycles to failure for the current stress range and is determined by the $\mathrm{SN}$ curve. The total damage after $N$ cycles is then found using:

$$
D_{t o t}=\sum_{i=1}^{N} \frac{1}{N_{F, i}}
$$

Failure happens when the total damage reaches the failure criterion $\Delta$ which in design is $\Delta=1$. Thus, the limit state equation for fatigue failure can be specified as:

$$
g=\Delta-D_{t o t}
$$

\section{Fatigue loading}

In order to use the design equations described above, the probability distribution for the stress ranges is needed. For wind turbines, the distribution depends on the loads from wind and waves, the dynamic properties of the wind turbine, and the control system. Furthermore, it depends on the number of hours in which the wind turbine is in the various operational conditions, for example production, idling, standstill, and yaw misalignment. For each condition, an aeroelastic code such as HawC can be used to solve the equations of motion for the wind turbine model, see (DNV/Ris $\varnothing 2002)$. The results of the calculations are the time series for the stresses in the wind turbine components. The stress distribution can be calculated based on the time series using various counting methods, for instance the rain-flow counting method. For operation in each of the stationary conditions, the stress distribution can often be approximated by a Weibull distribution. The distributions from all operational conditions should be combined in order to form an overall distribution for the loads on the components. Here a fit to a Weibull distribution is often used as well (DNV/Ris $\varnothing$ 2002).

\section{Uncertainties in fatigue design models}

In general, the uncertainties on SN curves can be put into three categories: fatigue modeling, fatigue resistance, and fatigue loading (Straub 2004). The uncertainties related to the fatigue modeling are caused by the use of the empiric SN curves and the Palmgreen-Miner damage accumulation law. The uncertainty of the SN curves is usually modeled by considering $C$ as a lognormal distributed random variable. For experiments performed under equal 
conditions, there will be variability in the results, which is caused by the natural variability in the fatigue resistance of apparently equal components. However, optimally, the experiments should be performed under varying conditions that reflect the variation for the components for which the same curve will be applied. Hereby, the statistical variation from the experiments can be used to estimate the joint uncertainty arising from the use of SN curves and for the fatigue resistance.

The Palmgreen-Miner damage accumulation law assumes linear damage accumulation and that the order in which the stress cycles occur does not influence the total damage. In general, these assumptions are not true, and this gives rise to uncertainty. This uncertainty is often taken into account by considering the failure criterion $\Delta$ as a stochastic variable. This uncertainty can be quantified if a large number of experiments are made with different, representative load histories.

For the fatigue loading, there is uncertainty on the parameters of the Weibull distribution and on the validity of the fit to the Weibull distribution. These uncertainties can be modeled by treating the scale parameter in the Weibull distribution as a random variable. This includes uncertainties on the load effects due to inaccuracies in the aeroelastic models.

\subsubsection{Fatigue for wind turbine components}

The SN model described above applies for fatigue crack growth in metals, but similar equations and models are used for mechanical wind turbine components such as gears and bearings. For gearboxes, the design requirements are given in the standard IEC 61400-4 (2012).

\section{Gears - pitting resistance}

For gears, there are several failure modes. One of them is pitting and concerns the surface durability. Design requirements for pitting resistance of gears are given in ISO 6226-2 (1996).

The surface durability is determined from an SN curve of permissible contact stress, $\sigma_{H P}$, as function of the number of load cycles $N_{L}$. Miner's rule is applied to assess the damage increment from each cycle based on the contact stress at the pitch point or at the inner point of single pair tooth contact $\sigma_{H}$ (ISO 6226-6 2006).

SN curves have been derived from tests of standard reference gears and can be converted to a specific application based on influence factors for lubricant, $Z_{L}$, pitch line velocity, $Z_{V}$, flank surface roughness, $Z_{R}$, work hardening, $Z_{W}$, and size, $Z_{X}$. The influence of the material is included in the allowable stress number $\sigma_{H, l i m}$ given in ISO 6226-5 (2003) for various materials as function of the surface hardness.

The SN curve can be drawn when the permissible contact stress is found 
for a reference case $\sigma_{H P r e f}$, where the life factor $Z_{N T}$ is equal to 1 , and for static stress $\sigma_{\text {H Pstat }}$ from the expression:

$$
\sigma_{H P}=\frac{\sigma_{H, l i m} Z_{N T}}{S_{H, l i m}} Z_{L} Z_{V} Z_{R} Z_{W} Z_{X}
$$

where $S_{H, \text { lim }}$ is the minimum required safety factor.

The limit state equation can be established as for crack growth. Failure is often defined as the formation of the first surface pits, if no pitting is acceptable. The formula assumes appropriate lubrication conditions. For probabilistic calculations, the uncertainties on the parameters should be taken into account.

\section{Bearings}

For bearings, the life equation, which corresponds to an SN curve is given by:

$$
L_{10}=\left(\frac{C}{P}\right)^{p}
$$

Where $L_{10}$ is the basic rating life, $C$ is the basic dynamic load rating, and $P$ is the dynamic equivalent load. In ISO 281 (2007), the exponent $p$ is equal to 3 for ball bearings and 10/3 for radial roller bearings. The basic rating life is the number of millions of revolutions that results in failure in $10 \%$ of the bearings.

The dynamic equivalent load is found as the cubic mean load (DNV/Ris $\varnothing$ 2002, p.135)(for $\mathrm{p}=3$ ), which corresponds to using Miner's rule for damage accumulation.

The modified rating life, $L_{n m}$, is given by:

$$
L_{n m}=a_{1} a_{I S O} L_{10}
$$

where $a_{1}$ is a life modification factor for other levels of reliability than $90 \%$, as given in ISO 281 (2007, table 12). The factor $a_{I S O}$ depends on lubrication, environment, contamination particles, mounting, and the ratio between the fatigue load limit and the dynamic equivalent load. Under favorable operation condition (extreme cleanliness and high viscosity ratio), the bearing life can be almost infinite, if the loads are smaller than the fatigue load limit.

Using the modified rating life, the limit equation can be expressed as:

$$
g=L_{n m}-L_{t o t}
$$

where $L_{t o t}$ is the number of revolutions. 


\section{$2.2 \quad$ Fatigue models with measurable damage size}

In the SN models, a damage increment was defined, but this is not a measurable damage size, and thus it is not directly coupled to the probabilities of the outcomes from the indicators. For fatigue, a fracture mechanical (FM) model can be used to describe the crack growth, and this will be described in the following. BS7910 (2005) represents the state-of-the-art for fracture mechanical models.

\subsubsection{Fracture mechanical model}

FM models are mainly based on the theory of linear elastic fracture mechanics. These models describe how growth of cracks depends on load cycles. During the life of a crack, it can progress through three different states: initiation, propagation, and failure. The initiation state is highly dependent on the quality of the material and the workmanship because small imperfections in the material can develop into cracks. Cracks can initiate in several ways, and often this initiation state is modeled indirectly by a number of stress cycles to initiation, $N_{0}$, and an initial crack size $a_{0}$. Crack propagation is dominated by the local stress field at the crack tip, and is perpendicular to maximum principal stress range.

The propagation state is described by Paris' law, which states the relationship between the crack growth rate, $d a / d N$, and the stress intensity range, $\Delta K$ (Anderson 2005) (Paris, Gomez, and Anderson 1961):

$$
\frac{d a}{d N}=C \cdot \Delta K^{m}
$$

The constants $C$ and $m$ can be found from experiments. The stress intensity range is a function of the crack size and the stress ranges, but only for simple cases the relationship can be found analytically. For real cases, finite element modeling can be used to derive an empirical relationship. Often, however, the influence of the boundary conditions is approximately taken into account by a geometry factor, $Y(a)$, and the following expression for $\Delta K$ is used, where $\Delta S$ is the stress range:

$$
\Delta K=\Delta S \sqrt{\pi a} \cdot Y(a)
$$

If the stress ranges can be assumed to follow a Weibull distribution, and $Y$ is assumed to be independent of the crack size, the differential equation can be solved analytically and has the following solution:

$$
a=\left(a_{0}^{\frac{2-m}{2}}+K A^{m}\right)^{\frac{2}{2-m}}
$$

where

$$
K=C N \Gamma\left(1+\frac{m}{B}\right) Y^{m} \pi^{\frac{m}{2}}\left(1-\frac{m}{2}\right)
$$


$A$ and $B$ are, respectively, the scale and the shape parameter of the Weibull distribution. If $Y$ is assumed to be equal to 1 , it corresponds to a semielliptical crack in a plate of infinite width subjected to normal stress perpendicular to the crack.

The uncertainty of the model can be taken into account by treating some of the parameters as random variables.

\subsubsection{Calibration of SN model to FM model}

FM models describe the fatigue growth of cracks and can be used for riskbased maintenance planning. However, the parameters in the FM model for a specific failure mode are generally not known, as the components are usually designed using SN models. A solution to this is to calibrate the parameters in the FM model based on the SN model. This calibration can be performed in various ways, but the most consistent method is a calibration which provides same reliability for the two models as described in Straub (2004).

The probabilistic SN model can be used to calculate the failure probability at all times during the lifetime, and the same applies to the probabilistic FM model. Consequently, the calibration can be described as an optimization problem, where the optimal values of the non-fixed parameters are found, such that the sum of the squares of the differences between the two models is minimized for a discrete number of time steps. One approach is to optimize with respect to the failure probability; an alternative approach is to use the reliability index, $\beta$, which can be found based on the probability of failure, $P_{f}$, as follows:

$$
\beta=-\Phi^{-1}\left(P_{f}\right)
$$

where $\Phi$ is the cumulative distribution function for the standard normal distribution. The reason for minimizing the differences between the reliability indexes instead of doing it for the probabilities of failure is that different orders of magnitude are equally important (Straub 2004).

The procedure for the calibration is as follows. First, a discrete number of time steps is chosen, and the reliability index is calculated at each time step for the SN model. The reliability index can be evaluated using First Order Reliability Methods (FORM). Secondly, the FM model is established, fixed parameters are chosen, and the optimization variables are set to initial values. The reliability index is then calculated for the FM model using FORM methods. Finally, an optimization algorithm is applied in order to find the optimal values of the optimization variables, such that the best fit is obtained.

In paper 11, optimization was performed with respect to the variables $a_{0}$ and $C$, and $m$ was adjusted manually to obtain the best fit. 


\section{Chapter 3}

\section{Updating of deterioration model}

The second step is updating the deterioration model using indicators and calculating the probability of failure for the updated model. In addition to the deterioration model, an indicator model is needed.

The indicators can range from accurate direct measurements to more uncertain indirect observations through for example vibration measurements or oil particle counting. Here, all types of observations are called indicators, which means that they are not perfect observations, but instead subject to uncertainties.

\subsection{Indicator models}

Indicator models define the probability of each condition monitoring outcome, as function of the damage state. For crack growth, a probability of detection (PoD) curve is often used for inspections. An example of such a PoD curve is:

$$
\operatorname{PoD}(D)=1-\exp (-D / \lambda)
$$

where $D$ is the crack size, and $\lambda$ is the mean size of detectable damages.

A PoD curve is a sufficient description if the decision rule only depends on whether damage is detected. In case the damage size is also of importance for the decision, a model for the measurement accuracy is needed. For example, an additive or multiplicative measurement accuracy can be used for continuous indicators. For indicators with discrete outcomes, models similar to the PoD curve can be used, e.g. the models used in paper 11 .

For online condition monitoring measurements, there are often more indicator outcomes for each time step. In this case, a statistical value such as the mean or maximum value can be used. 


\subsection{Bayesian updating}

Bayesian updating can be performed based on Bayes' rule. For events $A$ and $B$, Bayes' rule is given as:

$$
P(A \mid B)=\frac{P(A) P(B \mid A)}{P(B)}
$$

where $P(A \mid B)$ is the posterior probability of $A$ given $B, P(A)$ is the prior probability of event $A, P(B \mid A)$ is the likelihood of $B$ given $A$, and $P(B)$ is the probability of $B$.

Bayes' rule can also be applied to probability distributions. The classical problem is to obtain knowledge about an unobserved quantity $\theta$ through an observed quantity $y$. The prior density for $\theta$ is $p(\theta)$, and the relationship between the observed and unobserved quantity is expressed through a sampling model, $p(y \mid \theta)$, also called the likelihood function. The joint distribution of $\theta$ and $y$ can be evaluated as:

$$
p(\theta, y)=p(\theta) p(y \mid \theta)
$$

The posterior distribution is given as:

$$
p(\theta \mid y)=\frac{p(\theta) p(y \mid \theta)}{p(y)} \propto p(\theta) p(y \mid \theta)
$$

where $p(y)$ is constant for a given observation $y$, and is therefore just a normalization factor.

According to equation 3.4, the posterior distribution can be found as the product of the prior distribution and the sampling model (non-normalized). For some choices of prior and sampling model, the posterior distribution is of the same type as the prior distribution, and the parameters can be found analytically from the parameters of the prior and sampling distributions. Such a class of prior distributions for a sampling model is called conjugate. For example, if $y$ is normal distributed with mean $\theta$, and the prior for $\theta$ is normal, then the posterior will also be normal.

Often, the expected value of the posterior is of interest, which requires taking the integral of equation 3.4. For conjugate choices of distributions, the expected value can be evaluated based on the parameters. But for non-conjugate choices of distributions, other methods are needed for the evaluation of the posterior and the expected value of it. Two possibilities are sampling based methods and discretization. Both can be combined with graphical models called Bayesian networks.

\subsection{Bayesian networks}

Bayesian networks were developed for artificial intelligence within computer science. They are named after Bayes' rule, which is the basis for updating 
of probabilities within Bayesian networks, also called inference. Bayesian networks are graphical models consisting of nodes and directed links. No path in a Bayesian network is allowed to be cyclic, thus the network needs to be a directed acyclic graph (DAG).

Each node represents a stochastic variable, and the links between the nodes represent causal relationships. Thus, a link from $A$ to $B$ means that $A$ is a cause of $B$. The node $A$ is called a parent of $B$, and $B$ is a child of $A$.

For Bayesian networks, the probability distributions are defined for each node conditional on the parents. Therefore, the joint distribution of a network with $n$ nodes can be found using the chain rule as:

$$
p(V)=\prod_{i=1}^{n} p\left(A_{i} \mid p a\left(A_{i}\right)\right)
$$

where $p\left(A_{i} \mid p a\left(A_{i}\right)\right)$ is the distribution for node $A_{i}$ conditional on the parents of $A_{i}$. For nodes with no parents, their marginal distributions are used.

An introduction to Bayesian networks can be found in Murphy (2001a), and in Jensen and Nielsen (2007). Various software programs are available for computing inference in Bayesian networks. Examples are WinBUGS, The Bayes Net Toolbox for Matlab, and Hugin. See Murphy (2001b) for an extensive list of programs.

\subsubsection{Conditional independence}

The arrows in Bayesian networks represent conditional dependencies, and thus conditional independencies can be evaluated from the DAG. However, conditional dependencies can change, when variables are instantiated (observed). The effect of an instantiation depends on the connection type.

In figure 3.1, a serial connection is shown. The DAG can be read as $\mathrm{X}$ causes $\mathrm{Y}$ which causes $\mathrm{Z}$. If the state of $Y$ is not known, $X$ and $Z$ are dependent of eachother, and knowledge about the state of $X$ will change the probabilities of the states of $Z$. If, on the other hand, $Y$ has received evidence, the connection between $X$ and $Z$ is blocked, and they are conditional independent given $Y$.

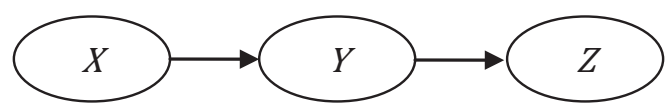

Figure 3.1: Serial connection.

A diverging connection is shown in figure 3.2, where $Y$ causes $X$ and $Z$. This connection type has the same properties as the serial connection. Knowledge of $X$ gives indirect information about $Y$, and thus information about $Z$. However, if $Y$ is already known, the indirect information from 
$X$ does not give more information about $Z$, and $X$ and $Z$ are conditional independent given $Y$.

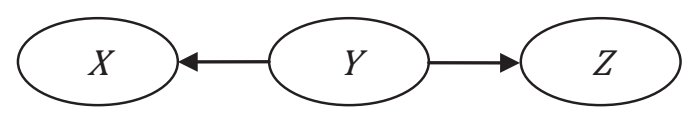

Figure 3.2: Diverging connection.

The last connection type is the converging connection shown in figure 3.3, where $X$ and $Z$ are causes of $Y$. Here, $X$ and $Z$ are conditional independent, if $Y$ has not received evidence. But if $Y$ has received evidence, conditional dependency is introduced between $X$ and $Z$. Since both $X$ and $Z$ are causes of $Y$, knowledge about one of them can explain the observed state of $Y$, and hereby give indirect information about the other. In fact, evidence can be transmitted between $X$ and $Z$ if $Y$ or a descendant of $Y$ is known. This is called the explaining away effect.

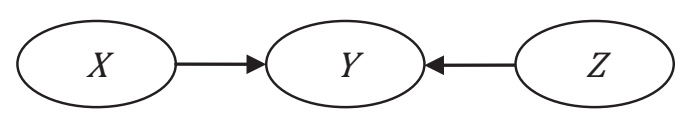

Figure 3.3: Converging connection.

From these connection types, it can be seen that the connection between two nodes is blocked if on all paths between the variables either:

- A variable is instantiated in a serial or diverging connection.

- There is a converging connection and no descendants of the variable in the connection are instantiated.

If all paths between two variables are blocked, they are said to be d-separated. A variable is conditional independent on all other variables if the parents, children, and parents of children are known. This set is called the Markov blanket.

\subsubsection{Sampling based inference}

Sampling based inference can be applied to networks with discrete as well as continuous nodes. Especially Markov chain Monte Carlo methods are interesting for inference in Bayesian networks. A Markov chain has the property that the stationary distribution is the posterior (Gilks, Richardson, and Spiegelhalter 1996). One Markov chain Monte Carlo method is Gibbs samling, see Hrycej (1990). Here, the realizations are drawn from the full conditional distributions. These include only the Markov blanket. For a node $v$, only the conditional distribution for $v$ given the parents of $v$, and the conditional distributions for the children of $v$ given their parents, are 
included, because $v$ is only included in these distributions, and the remaining conditional distribution are just constants:

$$
p(v \mid V \backslash v) \propto p(v \mid p a(v)) \prod_{w \in \operatorname{ch}(v)} p(w \mid p a(w))
$$

Gibbs sampling is performed by drawing samples from the full conditional distributions on turn. For a network consisting of three nodes, $X, Y$, and $Z$, the procedure is as follows:

$$
\begin{aligned}
X_{i} & \sim p\left(X \mid Y_{i-1}, Z_{i-1}\right) \\
Y_{i} & \sim p\left(Y \mid X_{i}, Z_{i-1}\right) \\
Z_{i} & \sim p\left(Z \mid X_{i}, Y_{i}\right)
\end{aligned}
$$

In the first round, initial values are used for the variables. A burn-in period is often necessary to obtain convergence to the true posterior distributions, and the samples from the burn-in are discarded. The expected value of the posterior distribution is then estimated as the expected value of a sample.

This method is implemented in the open source program OpenBUGS (Lunn et al. 2000). Here, it is possible to define logic nodes in addition to the stochastic nodes. The stochastic nodes are defined by their distributions, while logic nodes are defined as functional deterministic relationships of other nodes. When sampling from a network with logic nodes, the deterministic links are collapsed, and sampling is only performed from the stochastic nodes. If a stochastic node $A$ has a logic node $B$ as parent, the stochastic parents of the logic node $B$ are considered as parents for the stochastic node $A$.

\subsubsection{Inference in discrete Bayesian networks}

For a Bayesian network where all variables have discrete probability distributions, it is possible to calculate inference exact. For a network with $n$ variables, the joint distribution can be given as an $n$-dimensional probability table. In principle, the following procedure, described in for example Jensen and Nielsen (2007), can be used to calculate inference in any network with only discrete variables.

First evidence is inserted in the joint distribution:

$$
p(V, e)=\prod_{i=1}^{n} p\left(A_{i} \mid p a\left(A_{i}\right)\right) \prod_{j=1}^{m} e_{j}
$$

where $m$ variables have received evidence and $e$ is the evidence vector containing zeros and one.

The posterior distribution of $A$ given evidence, $e$, is calculated using Bayes' rule:

$$
p(A \mid e)=\frac{\sum_{V \backslash A} p(V, e)}{p(e)}
$$


In the numerator, the joint distribution of $A$ and $e$ is found by marginalizing out all other variables. This is a demanding task for large networks, as the full tables will have a very large number of entries. The marginalization procedure can be simplified using variable elimination, where one variable is eliminated at a time.

For example for the DAG in figure 3.1, the marginal distribution of $Z$ can be found as:

$$
p(Z)=\sum_{X} \sum_{Y} p(X) p(Y \mid X) p(Z \mid Y)=\sum_{X} p(X) \sum_{Y} p(Y \mid X) p(Z \mid Y)
$$

Here, $Y$ is eliminated before $X$.

An efficient version of the variable elimination method is the junction tree algorithm where the network is transformed into a undirected tree structure. The tree consists of cliques that consist of nodes from the original Bayesian network. There can be only one path between two nodes. To make it most computationally efficient, the cliques should be formed as small as possible.

To make a junction tree from a Bayesian network, a moralizing graph should first be made. For all nodes, links should be added between common parents, and the direction of all links should be removed. Next, the cliques are identified by successively eliminating nodes. A node can be eliminated if all neighbors are interconnected. If it is not the case, extra links should be added before eliminating a node. The eliminated node and neighbors then form a clique. The last step in creating a junction tree is to connect the cliques in such a way that all cliques in the path between two cliques include their intersecting nodes.

The junction tree algorithm is described thorough in Jensen and Nielsen (2007) and used in software programs such as Hugin.

\subsubsection{Dynamic Bayesian networks}

Dynamic Bayesian networks can be used to model variables that change with time. It typically consists of a number of equal time slices, which are connected only to the previous and following time slices. They are related to Markov processes, which fulfill the Markovian assumption: The future is independent on the past given the present. For a Dynamic Bayesian network, future time slices are independent of past time slices given the present time slice. A simple Dynamic Bayesian network is the hidden Markov model with unobserved variable $\theta$ and observed variable $y$, as shown in figure 3.4.

\section{Inference in dynamic Bayesian networks}

For dynamic Bayesian networks, the methods for inference used for normal Bayesian networks can be used. But other tailored methods originating from hidden Markov models are also available. Murphy (2002) presents several methods for inference in Bayesian network, and Straub (2009) presents 


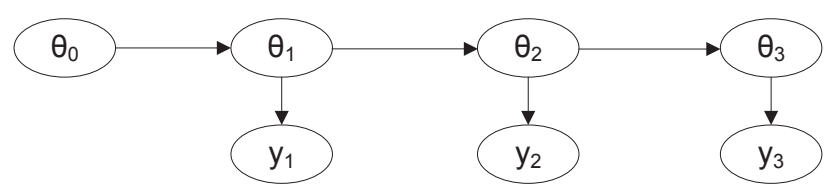

Figure 3.4: Dynamic Bayesian network corresponding to a hidden Markov model.

a method for deterioration modeling based on the Forward-backward and Frontier algorithm. For dynamic Bayesian networks, three tasks are relevant: filtering, prediction, and smoothing (Russel and Norvig 2003):

- Filtering is updating of the current state, given evidence up to the current state: $p\left(\theta_{i} \mid y_{1}, \ldots, y_{i}\right)$

- Prediction is updating of future states, given evidence up to the current state: $p\left(\theta_{j} \mid y_{1}, \ldots, y_{i}\right)(j>i)$

- Smoothing is updating of past states, given evidence up to the current state: $p\left(\theta_{i} \mid y_{1}, \ldots, y_{j}\right)(j>i)$

These tasks can be performed using forward and backward operations. The forward operation computes the posterior distribution for the current state, given evidence up to the current state, $p\left(\theta_{i} \mid y_{1}, \ldots, y_{i}\right)$, and can be applied directly for filtering. The forward operation is applied recursively in two steps:

$$
\begin{aligned}
& p\left(\theta_{i} \mid y_{1}, \ldots, y_{i-1}\right)=\sum_{\theta_{i-1}} p\left(\theta_{i-1} \mid y_{1}, \ldots, y_{i-1}\right) p\left(\theta_{i} \mid \theta_{i-1}\right) \\
& p\left(\theta_{i} \mid y_{1}, \ldots, y_{i}\right) \propto p\left(\theta_{i} \mid y_{1}, \ldots, y_{i-1}\right) p\left(y_{i} \mid \theta_{i}\right)
\end{aligned}
$$

First, the distribution for the current state is found, given evidence up to the previous time step, by calculating the joint distribution of the current and previous time step, and marginalizing out the previous time step. Next, Bayes' rule is applied to calculate the posterior distribution for the current state given evidence up to the current state, and using the result of the first computation as prior. For prediction, only the first step in the algorithm is used, as no evidence is available for future time steps:

$$
p\left(\theta_{j} \mid y_{1}, \ldots, y_{i}\right)=\sum_{\theta_{j-1}} p\left(\theta_{j-1} \mid y_{1}, \ldots, y_{i}\right) p\left(\theta_{j} \mid \theta_{j-1}\right)
$$

For smoothing, Bayes' rule can also be applied:

$$
p\left(\theta_{i} \mid y_{1}, \ldots, y_{i}, \ldots, y_{j}\right) \propto p\left(\theta_{i} \mid y_{1}, \ldots, y_{i}\right) p\left(y_{i+1}, \ldots, y_{j} \mid \theta_{i}\right)
$$


The first factor is the result of the forward operation. The second is the probability of future evidence, given the current state, which can be found by applying the backward operation:

$$
\begin{aligned}
p\left(y_{i+1}, \ldots, y_{j} \mid \theta_{i}\right) & =\sum_{\theta_{i+1}} p\left(\theta_{i+1}, y_{i+1}, \ldots, y_{j} \mid \theta_{i}\right) \\
& =\sum_{\theta_{i+1}} p\left(\theta_{i+1} \mid \theta_{i}\right) p\left(y_{i+1} \mid \theta_{i+1}\right) p\left(y_{i+2}, \ldots, y_{j} \mid \theta_{i+1}\right)
\end{aligned}
$$

The backward algorithm should be applied recursively, starting from the last time step, for which evidence has been received, whereby the last factor in equation 3.17 is equal to a vector of ones.

See Straub (2009) for a version of the algorithm, where the $\theta_{i}$ depends on other variables in addition to $\theta_{i-1}$.

\subsection{Application of $\mathrm{BN}$ to deterioration modeling}

Dynamic Bayesian networks have been applied to modeling of deterioration by Friis-Hansen (2000) and Straub (2009). The basis is a probabilistic model with a description of the damage size, such as the FM model presented in section 2.2. For a model with damage size, $D$, model parameter, $A$, observation, $I$, and failure probability, $F$, the network is shown in figure 3.5 .

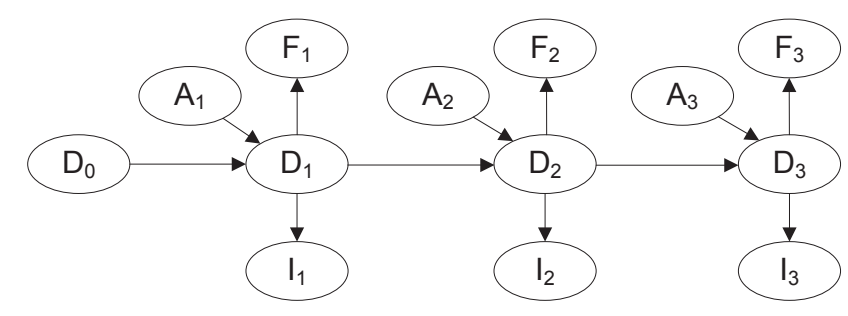

Figure 3.5: Bayesian network for deterioration modeling.

The network can be used for calculating the probability distribution for the damage size for each time step, based on a deterioration model. The deterioration model will often be a deterministic function $f$, and the uncertainties are expressed through stochastic model parameters:

$$
D_{i}=f\left(D_{i-1}, A_{i}\right)
$$

To include observations in the model, an observation model is needed. Here, the probabilistic of inspection/monitoring outcomes can be modeled by a probability density function conditioned on $D_{i}$ :

$$
p\left(I_{i} \mid D_{i}\right)
$$


Last, the distribution for the initial damage size should also be defined. The network can then be solved as a continuous network using sampling based methods or as a discretized network using exact inference methods.

\subsubsection{Continuous}

Sampling based approaches were previously considered unsuitable for deterioration modeling, partly because Gibbs sampling does not allow inclusion of deterministic relationships between nodes (Straub 2009). However, as explained in section 3.3.2, it is possible to include logic nodes, which deterministically depends on other nodes. When sampling from the network, these deterministic links are collapsed, and therefore the deterministic nodes cannot receive evidence. For deterioration modeling, damage growth is typically described by a deterministic model, and the nodes for the damage size can therefore be modeled by deterministic nodes.

During the lifetime, it should be possible to update the network using observations. This could be condition monitoring, inspections, load measurements, and the information that failure has not occurred. All these nodes should therefore be stochastic such that evidence can be entered.

The failure criterion is often described by a deterministic function:

$$
g=D_{\text {lim }}-D
$$

Failure occurs, when $g \leq 0$. The event of failure can be modeled by a binary node that returns 1 in case of failure $\left(D>D_{\text {lim }}\right)$, or else returns 0 . In WinBugs this can be modeled by a logic function:

$$
F=\left(\min \left(D, D_{\text {lim }}\right)==D_{\text {lim }}\right)
$$

The state of this node depends deterministically on the node for the damage size, and therefore it cannot receive evidence. However, this can be overcome by adding an additional binary failure node, which follows a Bernoulli distribution with parameter $p=F$. This node is in principle stochastic, and can therefore receive evidence, even though the probability $p$ is either 0 or 1 , and the state therefore is given when the damage size is known.

Observation models are generally given as probabilistic relationships, and can therefore be modeled by stochastic nodes. In case the damage size is subject to a measurement error, the outcome can be modeled by a normal distribution with mean equal to the damage size, or another distribution, if appropriate.

The same is the case for measurement of the load. If deterioration is modeled by equation 2.11, the load cycles are assumed to follow a Weibull distribution. If the load cycles are measured in each time interval, the scale parameter, $A$, in the Weibull distribution can be updated. 
If the outcome of an inspection is just detection or no detection, the probabilities of detection can be calculated using a $P o D$ curve such as equation 3.1. The value of the $P o D$ can be included as a deterministic node at each time step, and the inspection outcome is then Bernoulli distributed with parameter $p$ equal to the $P o D$.

The sampling based approach was used in papers 2 and 13 using the program OpenBugs (Lunn et al. 2000).

\subsubsection{Discrete}

The approach where continuous variables were discretized was used by both Friis-Hansen (2000) and Straub (2009). For discrete Bayesian networks, all nodes are stochastic and specified through their probability mass function conditioned on their parents.

\section{Discretization}

The selection of discretization scheme has significant influence on the accuracy of the results. Both the number of states and length distribution of states are important. Different variables could have different discretization scheme, but for simplicity, the same discretization should be chosen for all time slices. For variables with bounded ranges, these boundaries are also the outer boundaries for the outer intervals. For unbounded variables, the range should be chosen such that the probability of being outside that range is appropriately small.

The length distribution of intervals should be chosen based on the deterioration model. For a linear deterioration model (damage increases linearly with time), intervals of equal length are appropriate. For non-linear deterioration models, interval lengths can be assigned using a function $T_{x}$, which has a linear relationship with the damage model (Straub 2009). The inner interval boundaries $x_{B}$ of a variable $X$ can then be determined based on:

$$
x_{B}\left(2: m_{x}\right)=T_{x}^{-1}\left(T_{x}\left(x_{l b}\right): \frac{T_{x}\left(x_{u b}\right)-T_{x}\left(x_{l b}\right)}{m_{x}-2}: T_{x}\left(x_{u b}\right)\right)
$$

where $x_{l b}$ and $x_{u b}$ are the lower and upper boundary of the probable range of $X$, and $m_{x}$ is the number of states. For deterioration modeling Straub (2009) uses $T_{x}=\ln (x)$ and Friis-Hansen (2000) uses a power function.

\section{Calculation of probability distributions}

When the discretization scheme for the variables has been chosen, the discrete probability distributions should be determined. For Bayesian networks, the probability distributions are always given conditional on their parents. For a node with no parents, the probability distribution is a one-dimensional table, and for a node with $n$ parents, it is an $(n+1)$-dimensional table. 
For nodes without parents, distributions can simply be discretized by calculating the probability of being in each interval based on the original continuous distributions. For nodes with parents, the distributions need to be established for each possible configuration of the parents.

For damage growth, a deterministic function needs to be converted into a conditional probability distribution. For a node $D_{i}$, the state should be found for each configuration of parents. For each state of a node $D_{i-1}$, the state of the node $D_{i}$ at the following time step should be calculated, for each combination of states for the other parents. Generally, the state of $D_{i}$ will depend on whether the calculation is done based on e.g. mean, max, or min values in the intervals of the parents. For the best accuracy, sampling should be made from the intervals and the samples should be chosen uniformly from each interval, to remove bias from the prior distribution. The samples can either be chosen randomly or each interval can be divided into subintervals and the mean from each subinterval can be used. The latter seems to produce more accurate results at a lower number of samples. Sampling can also be used when the distribution is initially defined by a probability distribution conditioned on continuous variables.

The chosen discretization of nodes and the found conditional probability distribution should be verified using methods with known accuracy, for example Crude Monte Carlo sampling. The approach where continuous variables are discretized was used in papers $3,4,10$, and 11 . 



\section{Chapter 4}

\section{Risk-based decision making}

As described in section 1.4, decisions on operation and maintenance should optimally be made from a risk-based perspective based on the Bayesian preposterior decision analysis (JCSS 2008). This implies making decisions that minimize the total expected lifetime costs, taking uncertainties into account.

For the pre-posterior decision analysis, it is in principle possible to find an exact solution, where each decision is made taking the entire past into account. However, the problem becomes intractable due to the computation time being exponentially increasing with the number of time steps. One solution to this problem is the approach used for risk-based inspection (RBI), where the same decision rule is used at every time step, and the decision tree is reduced to a single branch, as explained in section 1.4.1. In this section, several approximative methods for risk-based decision making for offshore wind turbines are presented and compared; some of them use Bayesian networks. The methods are divided into three types: methods with fixed decision policies, methods using influence diagrams, and methods based on the partially observable Markov decision process (POMDP).

To estimate the total expected lifetime costs, it should be possible to calculate the probabilities and costs of inspections, preventive repairs, and failures. For general use, the following components should be included in the models:

1. Initial damage size

2. Deterioration

3. Failure

4. Repair after failure

5. Condition monitoring

6. Inspection scheduling/decision 
7. Inspections (outcome)

8. Preventive repair decision

9. Preventive repairs

10. Consequences/costs

Points 1-3 depend on the probabilistic deterioration model and the implementation of it that was described in chapters 2 and 3 respectively. Points 4 and 9 depend on the assumptions for repairs, and should be implemented accordingly. Points 5 and 7 depend on the indicator models used, as described in chapter 3. Points 6 and 8 depend on the method applied for making optimal decisions, and will be described in this chapter. Point 10 considers the modeling of consequences and costs. Here, the accuracy will depends on the time resolution used in the model. For real problems, discounting should be taken into account, and costs due to lost production should be included as well as the preventive maintenance costs and corrective maintenance costs. The subdivision of the costs will depend on the decision problem.

\subsection{Methods with fixed decision policies}

An approximation to the decision problem is the use of fixed decision policies that are constant with time. The accuracy of the approximation depends on the parameters used for the policies. If the entire past is included in the policies, the solution is exact, and the problem is generally intractable. Here, fixed policies/decision rules that depend on only one parameter are considered. The decision rules can be made with respect to either a directly observed variable or with respect to a value found by propagation of evidence (Bayesian updating). The first could be a constant or an inspection outcome and the latter could be the expected damage size, the probability of failure in following time step, or the probability of failure in the remaining lifetime. The decision rules based on propagation of evidence are better connected to the probability of failure, and they are expected to give a better solution closer to the true optimum. However, to use these decision rules, it is necessary to do an evaluation of expectation, where the rules based on directly observed variables are easier to optimize and easier to use in practice. Both approaches will be described in this section, and application examples are given.

\subsubsection{Decision rules based on constant or observed quantity}

For decision rules based on either a constant or a directly observed quantity, the optimal solution is found by optimizing with respect to a parameter for the decision rule. The total expected costs through the lifetime are 
evaluated for different values of the parameter, and the value yielding the lowest expected costs is chosen. Thereby, the same decision rule is used for all time steps.

Estimation of the expected costs requires calculation of the expected number of failures, repairs, and inspections during the lifetime. This can be done in several ways:

- Calculation of probabilities for each branch using structural reliability methods

- Crude Monte Carlo simulations during the lifetime

- MCMC Sampling in continuous Bayesian networks

- Exact inference in discrete Bayesian networks

\section{Calculation of probabilities for each branch using structural reli- ability methods}

The first method is the method used in the RBI framework described in section 1.4.1. The cornerstone in making this approach computationally feasible is to reduce the number of branches, such that the computation time is linear with the number of time steps. For RBI, failure was considered to be a terminal event, which is generally not the case for wind turbine components. However, the approach could be altered by using the same assumptions after failure as after preventive repairs, and thereby the method could be used for cases with inspections and a decision rule for repairs after inspections. The approach can be used for structural components, but also for some electrical and mechanical components, where the failure mechanisms can be formulated using mechanical models. This approach will not be considered further here.

\section{Crude Monte Carlo simulations during the lifetime}

The use of Crude Monte Carlo simulations of the lifetime is the same approach as used in simulation based approaches for estimating costs of corrective maintenance as described in section 1.3.1. The approach has been used for optimal planning of preventive maintenance in papers 1 and 8. The approach is not suitable for applications where the probability of failure is low, because too many simulations will then be necessary for an accurate estimation of costs. But the method can be used for components with higher failure rates. This type of model is very flexible, and repairs and inspections can easily be implemented. Real weather data can be used and lost production can be accurately modeled. However, when the model is very comprehensive, the computation time becomes long. 
In paper 8, the approach was used to find the optimal inspection interval, and the optimal threshold for the damage size for preventive repairs. When optimization is made with respect to two parameters, the joint optimum should be found. In figure 4.1, damage development is shown for the model when only corrective maintenance is used and when both corrective and condition-based maintenance are used. The optimum values can be found as the minimum values in figure 4.2 .
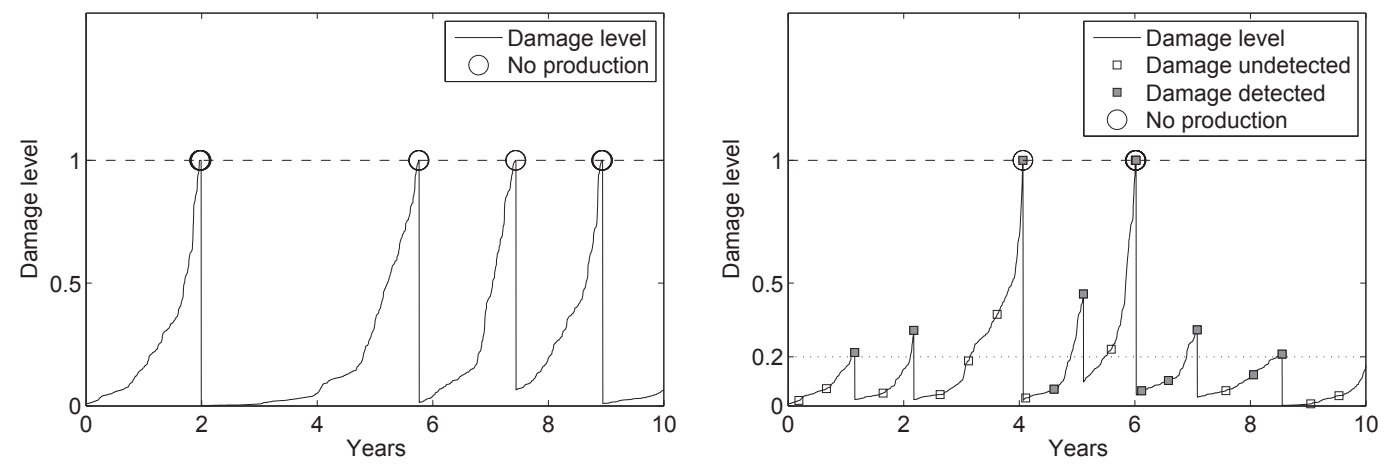

Figure 4.1: Damage development for corrective (left) and condition based maintenance (right) (paper 8).
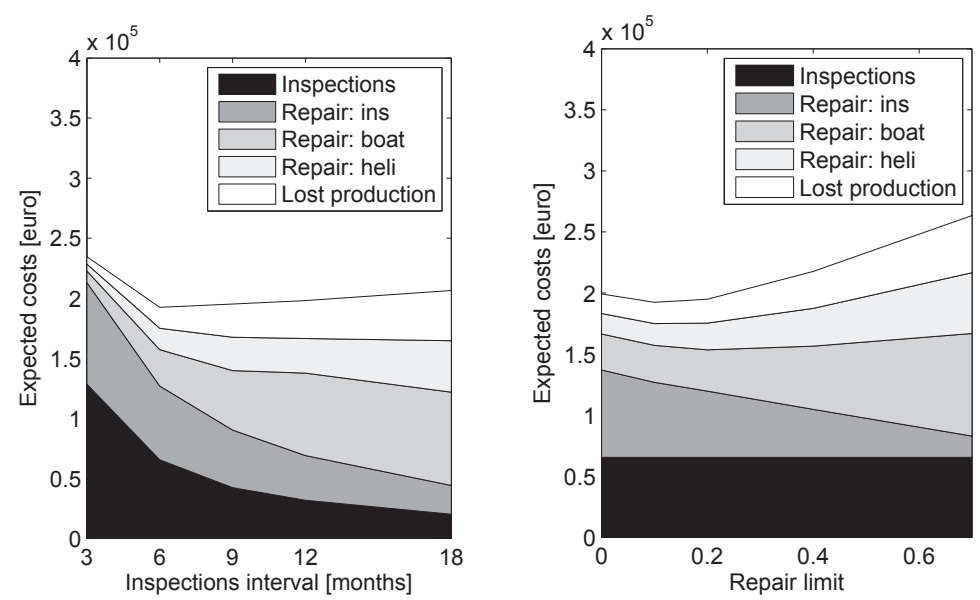

Figure 4.2: Expected costs for different inspection intervals and repair limits, divided into cost of inspections, cost of preventive repairs (Repair: ins), cost of corrective repairs performed by boat (Repair: boat) or helicopter (Repair: heli), and cost of lost production (paper 8).

\section{MCMC sampling in continuous Bayesian networks}

In this approach, MCMC sampling is used to estimate the expected number of failures, repairs, and inspections during the lifetime. These numbers 
can be estimated directly from the network if repairs and inspections are included in the network. The challenge is to include these things within the possibilities of MCMC.

This approach was used in paper 13, where OpenBugs was used for the model. The deterioration model was implemented as described in section 3.4.1. Three strategies were implemented, corrective maintenance, scheduled preventive maintenance and condition-based maintenance, as illustrated in figure 4.3 .
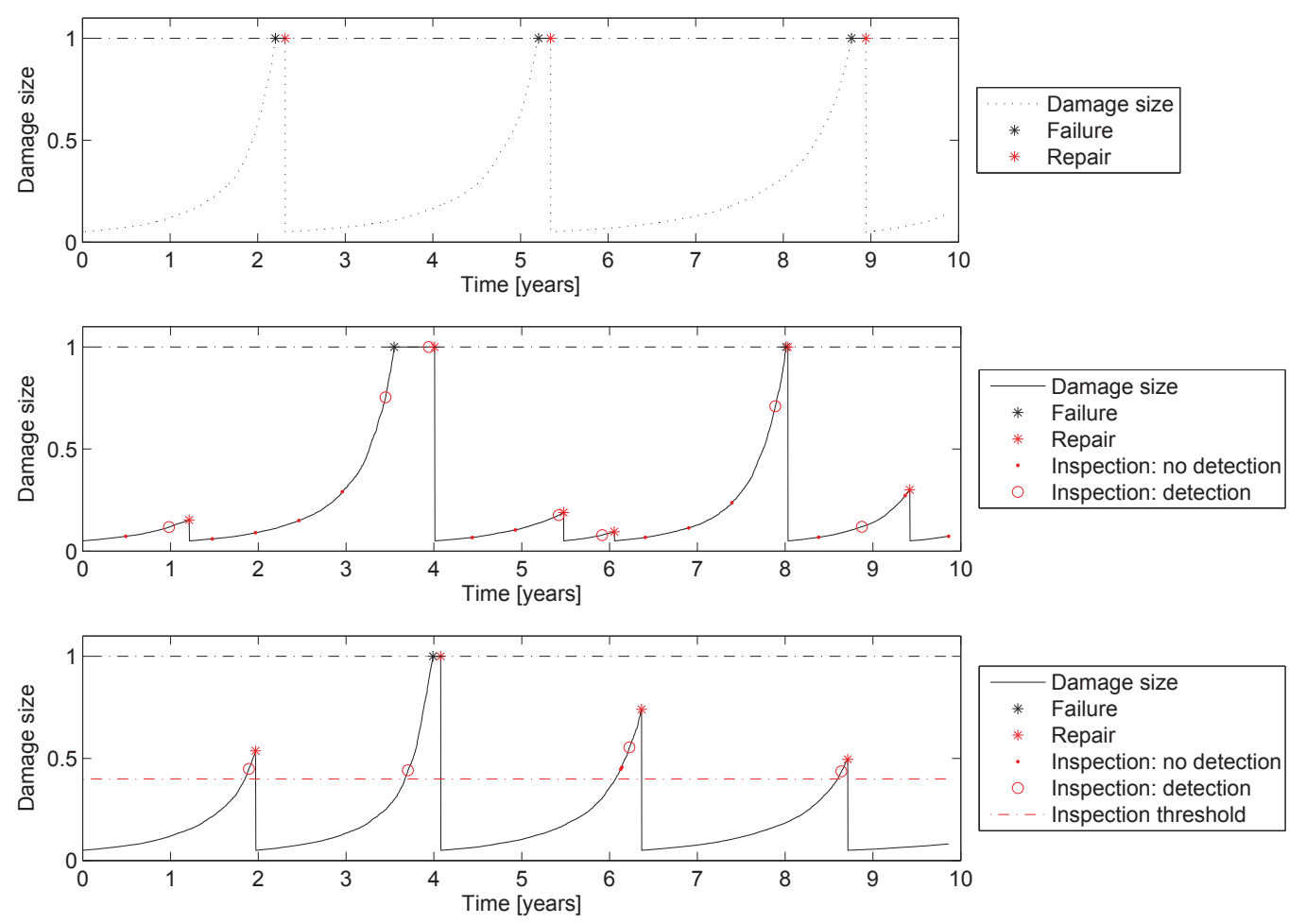

Figure 4.3: Corrective maintenance, timetabled preventive maintenance and condition-based maintenance (paper 13).

To model corrective maintenance, repairs need to be made after failure. Repairs can be implemented by including logic nodes $D_{F}(i)$ between the logic nodes $D(i)$, as illustrated in figure 4.4. The nodes $D(i)$ are calculated based on $D_{F}(i-1)$ using the deterioration model. The nodes $D_{F}(i)$ can be calculated by:

$$
D_{F}(i)=F(i) \cdot D_{0 R}+(1-F(i)) \cdot D(i)
$$

where $D_{0 R}$ represents the damage size after repair, and $F(i)$ is a binary failure node with the states 0 and 1 , where 1 means failure. Therefore, the damage size is changed to $D_{0 R}$ after a failure, otherwise it is not changed.

For scheduled preventive maintenance, scheduled inspections and preventive repairs in case of detection were modeled. For all time steps, a binary repair node $R$ was included. For the time steps with no inspections, 


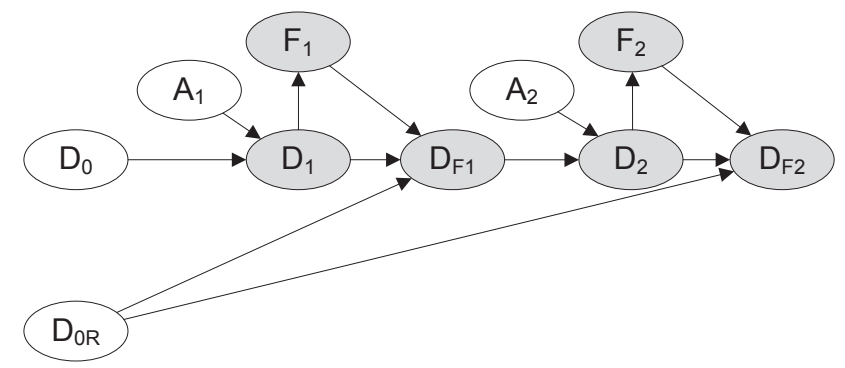

Figure 4.4: Bayesian network for MCMC based evaluation of total costs. The grey nodes are logic nodes and the white are stochastic.

it was set to a Bernoulli distribution with probability of repair equal to zero. For time steps with inspections, the probability of repair was first calculated in a logic node from a probability of detection curve such as equation 3.1. A repair was then made, if either $F$ og $R$ were equal to 1:

$$
D_{F}(i)=\max (F(i), R(i)) \cdot D_{0 R}+(1-\max (F(i), R(i))) \cdot D(i)
$$

For condition based maintenance, an online indicator is assumed to be available. The indicator is here modeled as a linear function of the damage size, but with a normal distributed error term. First, the value of the indicator excluding the error, $I_{\text {mean }}$, is found based on the damage size, and constants $k_{0}$ and $k_{1}$ from a deterministic relationship:

$$
I_{\text {mean }}(i)=k_{0}+k_{1} \cdot D(i)
$$

The actual value of the indicator, $I$, is modeled as a normal distributed variable with mean value $I_{\text {mean }}$ and standard deviation $\sigma$. An inspection is made if $I$ is above a threshold, $I_{c r i t}=k_{0}+x \cdot \sigma$, and the inspections are modeled in the same way as for scheduled preventive maintenance.

For scheduled preventive maintenance, optimization is done with respect to the inspection interval, and for condition based maintenance, optimization is performed with respect to the threshold parameter $x$. Figure 4.5 shows the optimization for each strategy, with the model used in paper 13.

\section{Exact inference in discrete Bayesian networks}

The approach used for sampling based continuous networks can also be applied to discrete Bayesian networks. Here, all nodes are discretized as explained in section 3.4.2, and exact inference can be performed. Similar inspection and condition monitoring models as used in the previous section can be applied. 

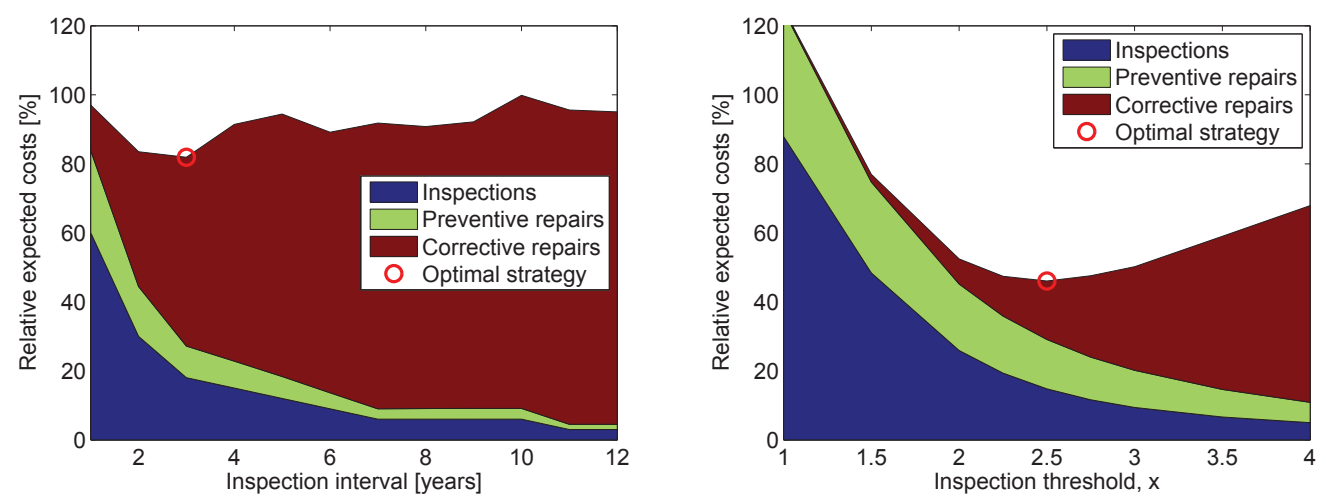

Figure 4.5: Total relative costs and optimal strategies for time-tabled and condition-based maintenance. The costs are shown relative to the costs of using only corrective repairs (paper 13).

\subsubsection{Decision rules based on quantity found using Bayesian updating}

Instead of using simple decision rules based on only one observation, the decision rules can be made with respect to, for example, the probability of failure. To apply the decision rule, Bayesian updating is necessary. To do this, Bayesian networks can be applied to deterioration modeling as described in section 3.4. The decision rule can be determined from simple considerations or by using simulation.

\section{Decision rules found using simple considerations}

The most simple way to make a decision rule is to take only the largest contributions into account and make a simple calculation of the break even point between to decision alternatives. This approach was used in papers 2 and 11 .

A simpel decision rule can be made based on the probability of failure, because the probability of failure is closely linked to the estimated cost of failures. In paper 2, the expected annual costs of failure and repair until the next repair were compared when a repair was performed at time $t$ or delayed until the following year $t+\Delta t$. The annual expected costs $E\left[C_{t}\right]$ and $E\left[C_{t+\Delta t}\right]$ are approximated by, respectively:

$$
\begin{aligned}
E\left[C_{t}\right] & =\frac{C_{R}}{t} \\
E\left[C_{t+\Delta t}\right] & =\frac{C_{F} P_{F}+C_{R}\left(1-P_{F}\right)}{t+\Delta t}
\end{aligned}
$$

Where $t$ is the time since last repair or failure, $P_{F}$ is the annual probability of failure in the following year, $C_{F}$ is the costs of failure, and $C_{R}$ is the cost 
of preventive repair. The break-even point is found where $E\left[C_{t}\right]=E\left[C_{t+\Delta t}\right]$. Solving for the annual probability of failure, $P_{F}$, yields:

$$
P_{F}=\frac{\Delta t}{t\left(\frac{C_{F}}{C_{R}}-1\right)}
$$

Thereby, a simplified decision rule for the probability of failure is found. The decision rule depends on the ratio between the cost of failure and the cost of preventive repair. This ratio, and $\Delta t$ is constant, and the found threshold value for preventive repairs thereby depends on the time since the last failure or preventive repair.

This decision rule gives an approximation on the annual probability of failure for a shorter period, but it does not assess the total annual life time costs due to the simple approach. In order to take all costs into account, simulation can be used.

\section{Decision rules found using simulation}

Simulation can be used to find the optimal value of a variable used for a decision rule. For constants or directly observable variables, the total expected costs for a certain value could be computed using a Bayesian network. However, if a threshold value for the probability of failure is used for the decision rule, the decision process cannot be modeled directly in the Bayesian network, because a calculation of expectation is necessary. Instead, Crude Monte Carlo simulations through the lifetime can be performed and a Bayesian network can be used in the simulations for updating the probability of failure. This requires that the Bayesian network is updated for each time step in the model for each simulation. This makes it a very time-consuming method.

This approach was used in paper 3 for decisions on inspections. If the probability of failure exceeded the threshold value, an inspection was performed. In case of detection, a preventive repair was made. Simulations were performed for different values of the threshold for the probability of failure, and the value yielding the lowest expected costs was chosen as the optimal value. The expected costs for different values of the threshold are shown in figure 4.6 .

\subsection{Influence diagrams, LIMID}

As an alternative to the use of fixed decision policies, influence diagrams can be used. An influence diagram is a graphical representation of a decision problem, in the same way that a Bayesian network is a graphical representation of a probabilistic model (Jensen and Nielsen 2007). In addition to the stochastic nodes (oval shape), an influence diagram also contains decision 


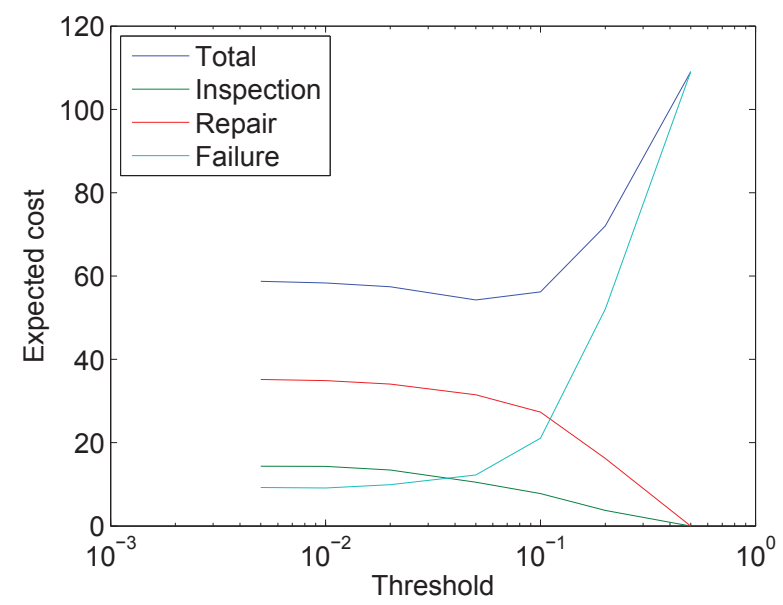

Figure 4.6: Total costs for different values of the threshold for the probability of failure.

nodes (rectangular shape) and cost nodes (diamond shape). The decision nodes contain policy tables, which specify the optimal decision for each possible configuration of past evidence. In practice, such a decision problem becomes intractable, as the decision node will be $n$-dimensional, where $n$ is the number of past nodes that can receive evidence (Jensen 2008).

As an approximation, a limited memory influence diagram (LIMID) as proposed by Lauritzen and Nilsson (2001) can be used instead. Instead of including all past nodes in the decision table, only direct parents are included. The optimal decision policies can be found using a single policy updating (SPU) algorithm. Here, the decision policies are updated successively until lower total costs cannot be obtained by changing only one decision policy. Therefore, a local minimum is found, but it is not guaranteed to be the global minimum. When the model is made, care must be taken to ensure that the solution can converge to the global minimum. This puts limitations on the complexity of decision problems that can be modeled.

This approach was used in paper 10 for decisions on repairs and in paper 3 for decisions on inspections. For decisions on repairs, the model could be made by only including the most recent inspection outcome when a decision is made. However, to ensure convergence to the global minimum, it was found necessary to add the previous decision node as parent to the decision node as well, as shown in figure 4.7 .

When the optimization problem is solved, the optimal decision for each time step is found dependent on the parents of the decision nodes. When the states of the parents are known, the solution tells what decision to make at each time step. The policies will generally not be stationary with time and will often be different near the end of the lifetime compared to earlier in the lifetime. 


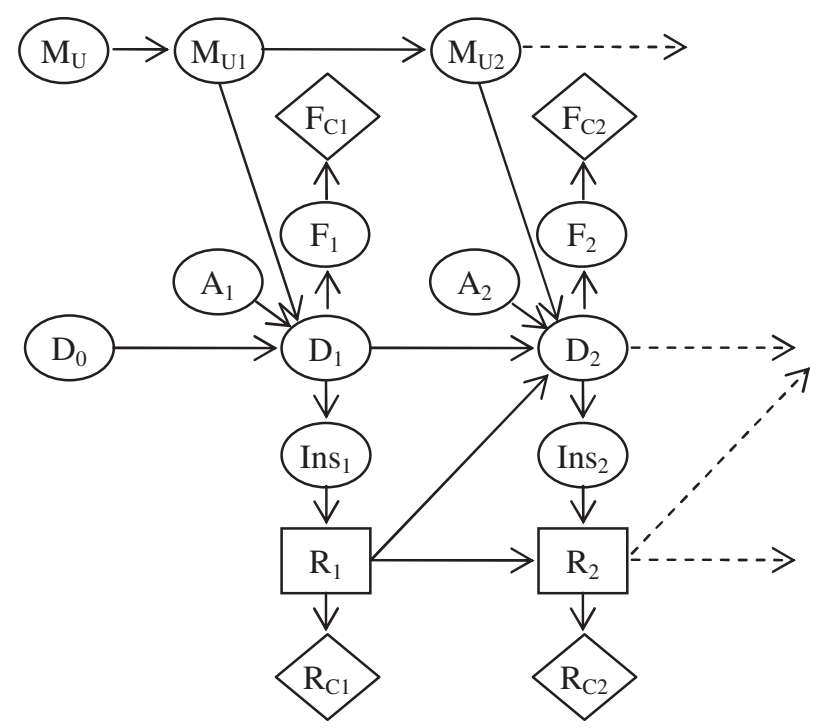

Figure 4.7: Influence diagram for decisions on repairs (paper 10).

With this approach, only the parents of the decision nodes are taken into account when a decision is made. Instead, evidence can be entered continually during the lifetime, and before each decision is made, the decision policies are updated. In this way, the entire past is always taken into account for the current decision. However, the optimal decisions for future decisions are needed for calculating the total expected costs of the current decision alternatives, and for the future decisions, only the direct parents and the evidence entered until now are taken into account. Therefore, is it still an approximative solution.

In paper 3, the total expected costs were found using simulation for the case where the decision policies were updated at each time step. These costs were found to be $16 \%$ lower than the costs estimated by the network, when the initial decision policies were used throughout the lifetime.

\subsection{Markov decision processes}

The underlying assumption for Markov decision processes is the Markovian assumption of independence between the past and future given the present. The most basic type of Markov decision processes is the fully observed Markov decision process. Here, the state of the variable of interest is known with certainty at the current time step, when a decision is made. In figure 4.8, a simple example is shown where the damage state is directly observable at each time step, and decisions on repair are made.

To solve a Markov decision problem efficiently, the Markov assumption can be exploited. Because the future only depends on the current state and not the entire past, the optimal decision at each time step only depends on 


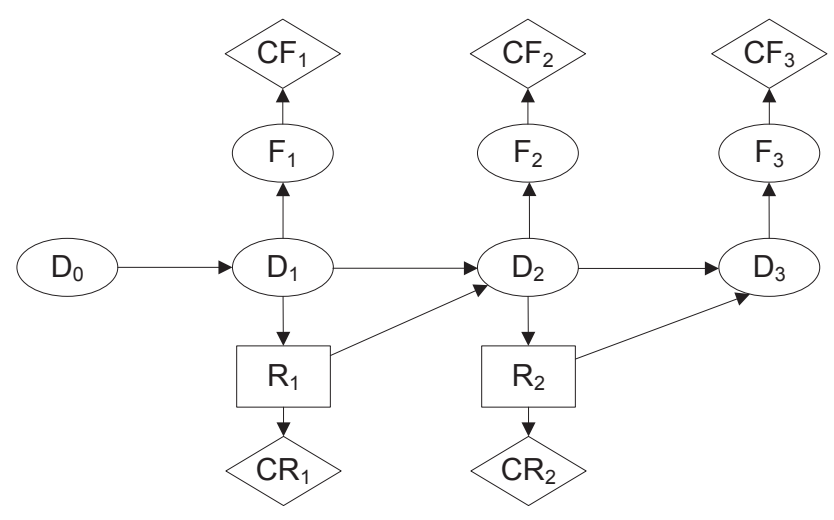

Figure 4.8: Fully observed Markov decision process.

the current state. Therefore, the optimal decision can be found for each time step for each possible state. Instead of evaluating all branches in a decision tree, the optimal decisions can be found using a recursive algorithm based on dynamic programming (Dasgupta, Papadimitriou, and Vazirani 2006). Starting from the last time step, the optimal decision and associated expected costs are found for each possible damage state. Then the computations are done for the second last time step, and here the results from the last time step can be used. Recursively, the optimal decision can be found for each time step in this manner, and thereby the computation time is linear with the number of time steps.

Often the damage state is not directly observable as only an indication of the damage state is available, for example an inspection or monitoring outcome. In that case, the problem can be modeled by a partially observable Markov decision process (POMDP), as illustrated in figure 4.9. Partially observable Markov decision processes have been used for maintenance planning by Corotis, Ellis, and Jiang (2005) and Faddoul, Raphael, and Chateauneuf (2011).

For a POMDP, the current damage state is not known. However, the knowledge about the current damage state, can be expressed as the belief state for the damage state, i.e. the probability distribution for the current belief state. Then, the future is independent of the past given the current belief state, and the optimal decision at a given time step only depends on the current belief state. Therefore, dynamic programming can be used as for the fully observed Markov decision process, by recursively calculating the optimal decision for each possible belief state. In reality, there are infinitely many possible belief states, as the belief state is represented by a continuous valued vector with the sum one. Therefore, the optimal decision and expected utility can be evaluated for a number of grid points, and interpolation can be used for finding the utility between states.

This approach was used in paper 5 for decisions on inspections and re- 


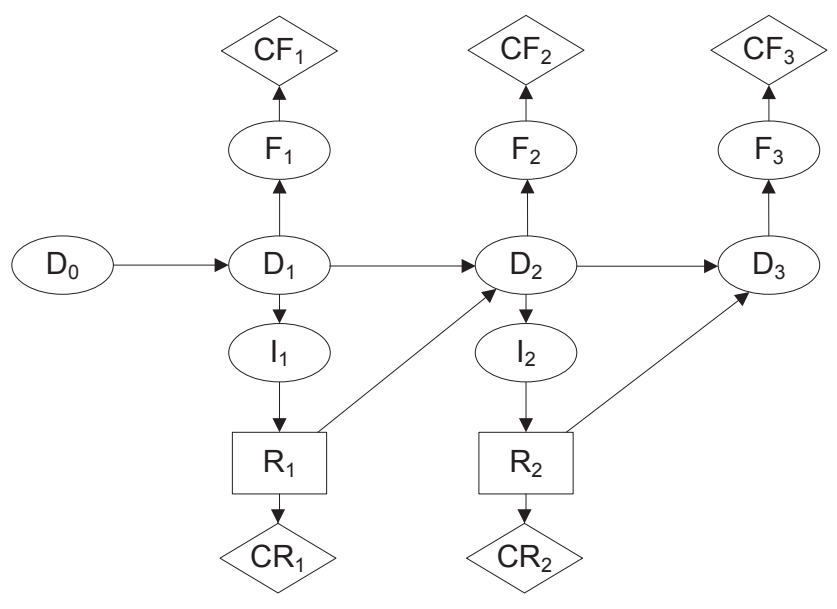

Figure 4.9: Partially observed Markov decision process.

pairs, and monitoring was assumed to be available. The damage node for the damage size had three states: healthy, damaged, and failed. The problem with the approach is that the computation time increases with the number of grid points considered. If the approach is to be used together with a deterioration model as described in section 2.2 , many damage states are generally necessary for accurate modeling. If the grid points are chosen arbitrarily, a huge number are necessary and the problem will become intractable. However, a potential solution is to consider only unimodal distributions and choose them based on parameters, for example mean, standard deviation and skewness.

A limitation of the approach is the Markovian assumptions of independence between future and past given the present. This means that epistemic time-invariant uncertainties are not taken into account. However, a variable for this uncertainty could potentially be included in the model, and the optimal decisions could be found for all possible combinations of the belief state for the damage and for this variable. This will significantly increase the computation time.

\subsection{System effects}

The optimization problems described so far have only considered one component in one wind turbine. For some decisions, a better solution can be obtained by including system effects in the decision problem, for example considering an entire wind farm with many components in each wind turbine. Various types of system effects can be considered. Here, correlations between variables are considered, as well as the influence of the number of inspections/repairs on the costs. 


\subsubsection{Correlations between variables}

Variables in deterioration models for the components in a wind farm will often be correlated. If the strength variables for a component are subject to epistemic uncertainty, parts of this uncertainty will typically be joint for that component in all wind turbines in the farm, or even for that component in wind turbines of the same type in different wind farms.

There will also be correlations on the loads. There are correlations between loads on turbines in the same wind farm, as the weather highly influences the loads. However, the load in each turbine highly depends on the topography of the wind farm and the wind direction, as the turbulence is increased in the wakes of turbines. On different components in the same turbine, there will also be correlations, but because the load effect on each component will be different, the correlations will vary.

These correlations between strength and load variables imply that there is correlation between the time to failure of the components. The correlations can be exploited by Bayesian updating. If a variable for the joint uncertainty is included in the model, this variable is updated when evidence is entered for any of the variables included in the model.

In paper 11, correlations were included between the epistemic model uncertainty, $M_{U}$, for each component of the same type in a wind farm. This was done by including a hyper parameter, $H M_{U}$, as a parent for the $M_{U^{-}}$ variables as shown in figure 4.10 , where $a_{i, j}$ is the damage size at time step $i$ for turbine $j$. In this way, inspections performed on one wind turbine also affected the reliability of the other turbines in the model.

\subsubsection{Influence of number of inspections/repairs on costs}

Another system effect is the influence of the number of inspections or repairs performed at the same time on the costs of the inspections or repairs. If several inspections can be performed during one day, the travel to the wind farm should only be paid once, and it will be beneficial to schedule several inspections the same day instead of distributing them. For some vessels such as crane ships, the mobilization costs can dominate the costs. If these costs are large compared to the unit repair costs, it might be beneficial to make additional repairs preventively when other repairs, corrective or preventive are made. In paper 11, decisions on preventive repairs were made each time a failure occurred, and the mobilizations costs would have to be paid anyways. The decisions were made based on simple cost considerations, and the benefit of using this strategy, instead of using only corrective maintenance, is shown in figure 4.11.

In paper 5, the mobilization costs were also assumed to be paid only once, if several repairs were scheduled at the same time step. If a corrective or preventive repair was scheduled for one turbine in the wind farm based 


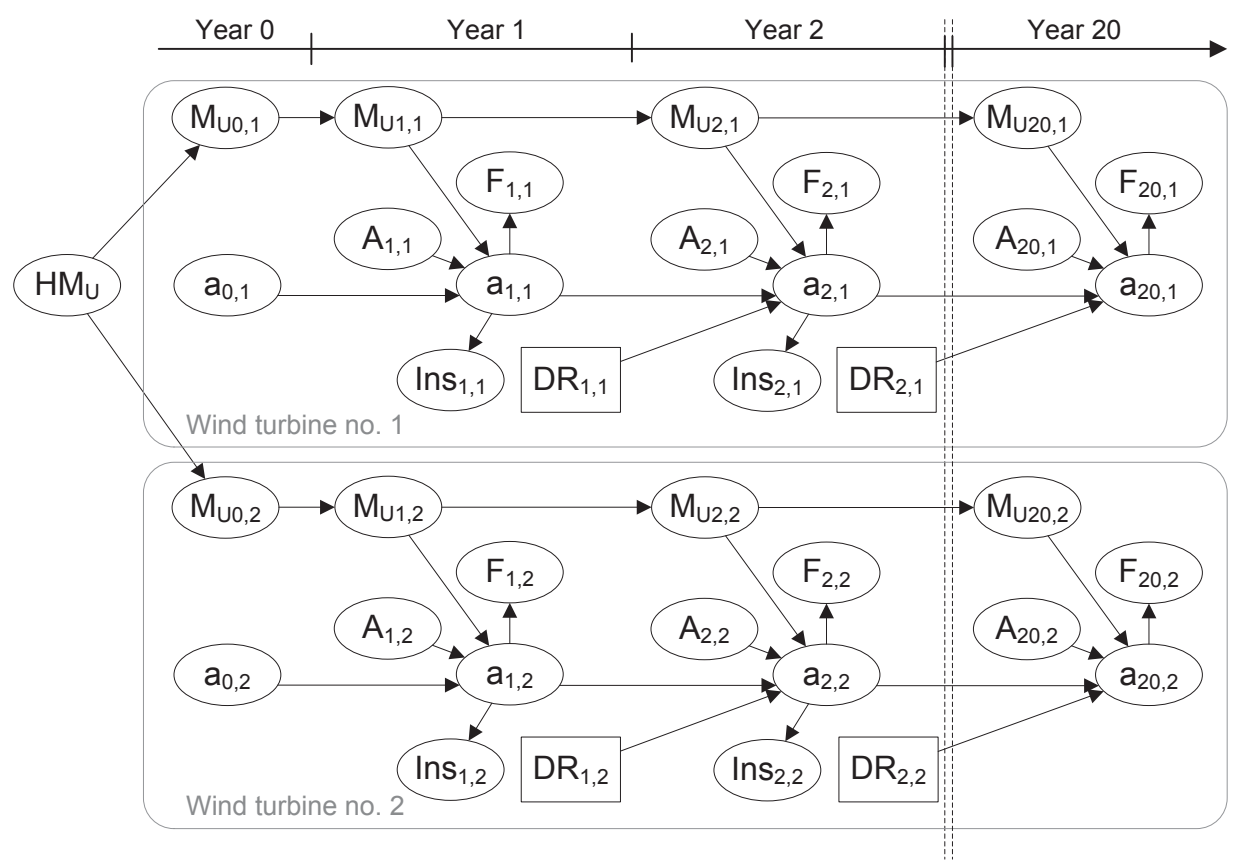

Figure 4.10: Section of Bayesian networks model for two wind turbines (paper 11).
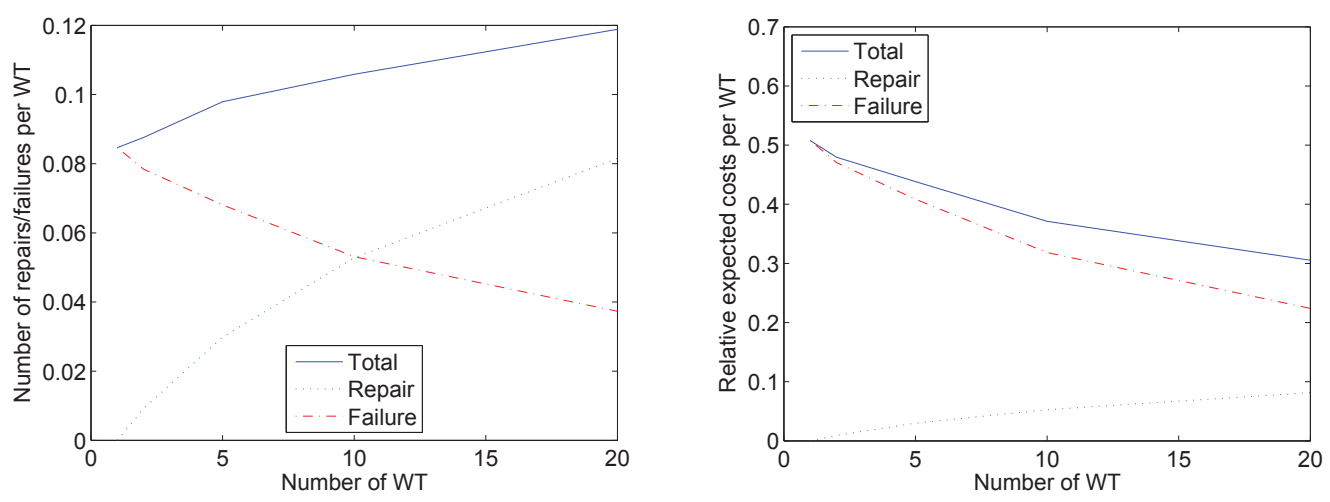

Figure 4.11: Expected number of failures and relative expected costs as function of the number of wind turbines in the wind farm (paper 11).

on the decision policies found from the POMDP, modified decision policies were used to determine whether or not additional repairs should be made. 


\section{Chapter 5}

\section{Developing models for real decision problems}

The models considered so far take their starting point in the models used in the design of a component. This approach is well suited for components where an available design model accurately describes the deterioration process and considers the uncertainties. However, for real life decision problems, this is not always the case.

Firstly, the design is made by turbine manufacturers or subcontractors, and the design calculations are not always available to a turbine owner who needs to make decisions on maintenance. The turbine owner also does not necessarily have access to accurate aeroelastic models of the turbines, needed to access the design loads on a component, or to accurate descriptions of the turbine components, needed to access the capacity. Therefore, it can be difficult for the decision maker to reestablish an accurate design model.

Secondly, even if the design models used in the design are available, the lifetime expected from the design models is often not realized in practice. Bearings, for example, are often designed such that $10 \%$ are expected to fail during the lifetime, but in real wind farms this number of failures may be reached much earlier in the lifetime. In such cases, it seems that the assumptions used in the design are not fulfilled in real life.

If the problem is that loads are higher than expected, monitoring can be used to measure the loads, and Bayesian updating can be applied to update the probabilistic models. However, this approach does not always help if the load on the component is correct, but conditions not taken into account in the design equations are affecting the degradation. For example, for bearings, standstill events can be of importance due to micropitting. Further, the lubrication is of large importance, and proper cleanliness conditions can be hard to ensure for offshore wind turbines. Furthermore, temperature affects the viscosity, and the effect is not always taken properly into account. In such cases, the models need to be improved to model the physics properly 
and to consider the special conditions causing deterioration.

It is far from trivial to establish improved models. The basis for improving the models will typically be empirical knowledge from experienced experts and operational data from wind farms with some years in operation. The data typically includes inspection and repair reports, SCADA data, and condition-monitoring data. As described earlier, Bayesian networks can be used for deterioration modeling and for updating of models. But it is also possible to use Bayesian networks for learning of models, and this option seems relevant to explore.

In addition to the deterioration model, an inspection/monitoring model is necessary for using Bayesian updating to update the deterioration model. Also, these models are often not available, and the option of using Bayesian networks to learn these models is therefore explored.

\subsection{Learning of models using Bayesian networks}

In this section, the possibilities of learning with Bayesian networks will be described. There are several cases to consider, which should be dealt with using different methods:

- Learning of parameters in a model

- Learning of models

- Learning of the causal relationships

In the first case, the model is known, for example by a deterministic equation, cf. equation 3.18, but some of the model parameters are not known. In the second case, no quantitative model for the relationship is known, but the locations of the links are known. In the third case, the locations of the links, i.e. the causal relationships, are not known. The three cases are described in the following sections.

\subsubsection{Estimation of model parameters from data}

The estimation of model parameters from data is different from the other cases, as it is actually not a learning problem. Instead, this problem can be solved using standard inference methods, either exact inference for discretized networks, or sampling-based inference for continuous networks.

To use this approach, a Bayesian network for the deterioration model needs to be established first. The network structure is repeated for each case where data is available. The networks are united in a joint network by adding a hyperparameter as a parent for all instantiations of the parameter that needs to be estimated. For this hyperparameter, a non-informative 
prior is used, for example a uniform distribution within the sample space, or a normal distribution with large variance.

This approach was used in paper 4 for estimation of the parameter $\lambda$ in the inspection model, in the case where the deterioration model was known. The approach can be used in a similar way for parameters in the deterioration model.

For discrete networks, using this approach for several parameters at the same time can be a problem, as the cliques of the junction tree become large, and the memory demand also becomes large.

\subsubsection{Estimation of model from data}

Estimation of the model from data can be done using the discrete network approach described in chapter 3. The problem is then a learning problem, where the aim is to learn the conditional probability distribution for one or more parameters. The expectation-maximization (EM) algorithm can be applied for this task, and the algorithm was described in paper 4. For a traditional Bayesian network, each parameter has its own conditional probability distribution. For deterioration modeling, dynamic Bayesian networks can be applied, where the conditional probability distribution for the instantiations of a node in all time slices will be the same. As described by Murphy (2002), the EM algorithm can be applied to those problems with straightforward extensions, and this can be implemented in the commercial software program Hugin by using objects.

The Bayesian network used for deterioration modeling in section 3.4 was a discretization of a continuous model, and the conditional probability distribution for the damage size was found using simulation from the deterministic deterioration model. This gives a characteristic structure of the conditional probability distribution with diagonal bands of the non-zero probabilities. However, the EM algorithm does not know that such a deterioration behavior is expected, and as the damage size will not typically be observed, the data does not give enough information to ensure such a behavior. Therefore, the algorithm will probably fail to give a reasonable conditional probability distribution, if the node for the damage size is a discretization of a continuous variable.

An option could be to use only a few discrete states to describe the damage state, and to choose these states based on the outcome of the available indicator. This means that the transfer from non-detectable to detectable damage is modeled by a geometrical distribution, and thereby the probability of transferring is constant for each time step, instead of increasing with time. However, even though this model may not be realistic, it might be the best alternative, if a better damage model is not available. 


\subsubsection{Estimation of causal relationships}

This case considers finding the causal relationships between parameters, based on a data set consisting of multiple coherent observations of the parameters. This corresponds to learning the network structure, and methods for doing so for discrete Bayesian networks are described in Heckerman (2008), Murphy (2002), and Jensen and Nielsen (2007). The starting point is a collection of nodes, and the algorithm finds the most probable links between the nodes, and therefore no prior knowledge is necessary.

For deterioration modeling, dynamic Bayesian networks can be used, which consist of a number of interconnected equal time slices. To learn the structure for a dynamic Bayesian network from data, it is necessary to set up some constrains. The links within each time slice should be equal for all time slices, and the links to the following time slice should be the same for all time slices.

This case shares the same limitations as described in the previous section, and will not be considered further here.

\subsection{Case study: pitch gear deterioration}

In order to test the overall approach presented in this thesis for a real decision problem, a case study was made in collaboration with the Energy Research Centre of the Netherlands (ECN).

The case considers deterioration of the internal gear of the blade bearing for turbines with an electrical pitch system. For a turbine, wear of the gear teeth was observed and preventive maintenance was performed on that basis. A measurement campaign was conducted for the turbine in a period overlapping the repair. Initial investigations of the data indicated that the maximum pitch motor toque reached when the turbine status changed from running to idling (and the blades were pitched from 0 to 70 degrees) could be used as an indicator for the deterioration of the pitch bearing gear. The maximum pitch motor torque was increasing in the period before the repair, and after the repair the values had decreased compared to the measurements before the repair, but seemed to have an increasing trend. Therefore, the pitch motor torque seemed to be a potential indicator for deterioration of the pitch gear.

The turbine for which measurements were available was an onshore multi-megawatt turbine. However, the idea was that the approach could be used for offshore wind turbines with electrical pitch systems, where inspections are more expensive. The pitch motor torque was expected to be proportional to the pitch motor current at constant pitching velocity, and the pitch motor current was known by the control system of the wind turbine and was included in the SCADA data. Therefore, the potential for a low cost online condition monitoring system based on pitch motor current 
measurements seemed to exist.

The intention of the case study was to establish a probabilistic damage model based on theoretical models, update the model using measurements of maximum pitch motor current, and use the model for risk-based decision making. In order to do update the model using the measurements, a probabilistic indicator model was needed, and the data was analyzed with the aim of developing such a model. The analysis revealed that the temperature and time since the last time the turbine was pitching (still time) were the main factors influencing the variation in the pitch motor current and torque. The current and torque were larger in case of larger still time and larger in case of lower temperatures. The influence of temperature caused seasonal variations in the current and torque, and as the repair was performed in the summer and caused a period with no measurements, and the apparent influence of deterioration was actually the influence of temperature. This investigation made it clear that this case study could not be used to demonstrate the approach.

The case study resulted in paper 12 , where a statistical analysis of the pitch motor current measurements was presented with the conclusion that the maximum pitch motor current was not a suitable indicator for the health of the teeth in the pitch bearing. Further analyses were presented in paper 6 , as measurements of pitch motor current and torque can still potentially be used to detect deterioration in the pitch system, for example in the pitch bearings, as increased bearing friction will increase the torque demand. The paper presents valuable knowledge for people trying to make a condition monitoring system for pitch systems. 



\section{Chapter 6}

\section{Conclusions and outlook}

The maintenance costs for offshore wind turbines are sizeable, and presently the maintenance effort is not planned using methods that fully exploit the available information. For oil and gas structures, risk-based methods based on the Bayesian pre-posterior decision analysis have been used. This approach is promising for planning of maintenance for offshore wind turbines, but needs to be adjusted because the assumptions behind the approximations normally used for oil and gas structures are not fulfilled. Approximations are necessary as it is computationally intractable to solve the decision problem exact.

The approach considered in this thesis is applicable to components exposed to deterioration processes. It consists of three parts: establishment of a probabilistic deterioration model, updating of the deterioration model using indicators, and risk-based decision making based on the updated model. The starting point for the deterioration model is the model used for the design. For fatigue, these models are generally empirical models based on SN curves and Miners rule for damage accumulation. As indicator outcomes generally depend on the physical damage size, the damage state included in the deterioration model should correspond to a physical damage size. This is generally not the case for fatigue design models, but instead fracture mechanical models calibrated to give the same reliability can be used.

For updating of the deterioration model, two methods based on Bayesian networks are examined. The first method uses Markov chain Monte Carlo methods for sampling in continuous networks. The deterioration model is built using a combination of stochastic and deterministic/logic nodes in the program WinBugs. In the other approach, discrete Bayesian networks are used. The continuous damage model is discretized, and the conditional probability distributions corresponding to the probabilistic damage models are found using samling. For both methods, the deterioration model can be updated using indicator outcomes.

Generally, decision problems with inspection and repair decisions were 
considered, and various approaches were considered for solving the decision problems:

- Methods with fixed decision policies

- Decision rules based on constant or observed quantity

- Decision rules based on quantity found using Bayesian updating

- Limited memory influence diagrams (LIMID)

- Partially observable Markov decision processes

Even though all methods consider a combination of monitoring/inspections and repairs, not all decisions strategies are optimized in all methods. Furthermore, there are differences in how they can be used in real life.

Decision rules based on a constant or observed quantity are simple to use in real life, as decisions can be made without consulting programs after indicator outcomes are received. Decision rules can be used for both inspections and repair decisions, and optimization is then performed for two parameters. Optimization can be done by calculating the expected costs for varying values of the optimization parameters (e.g. inspection interval or repair threshold) using structural reliability methods, simulation, or using either continuous or discrete Bayesian networks.

The decision rules based on a quantity found using Bayesian updating (e.g. probability of failure) are more cumbersome to apply, because they require updating of evidence before making decisions. They were applied for either inspection or repair, while a simple decision rule was used to model the other. The optimal value was estimated using simple considerations or found using simulations. The method with simple considerations is easy to implement, but will often give a crude approximation. On the other hand, the latter requires a quite advanced simulation procedure, as the Bayesian network used for probability updating needs to be updated during the simulations. For updating during simulations, discrete Bayesian networks were used because updating using evidence in those is very fast compared to the MCMC methods.

Limited memory influence diagrams are based on discrete Bayesian networks. When applying those, it is possible to use either original decision policies, calculated at the beginning of the lifetime, or to update the policies each time new evidence is received. The latter option is most precise, because typically only the most recent inspection result is be taken into account for future decision policies. On the other hand, all evidence that has been entered into the network is taken into account, and therefore it is more precise to update the policies every time new evidence is received. Only one type of decision is modeled using decision nodes, either inspections or repairs, as the SPU algorithm used for optimizing the decisions will most 
likely get stuck at a local minimum if both inspections and repairs are modeled using decision nodes. Instead, a simple decision rule can be used to model the other decision and can be optimized manually, either using costs that can be found approximately from the network, assuming no updating of policies, or they can be estimated using simulation, if policies are updated.

The methods based on Markov decision processes are based on discrete damage models. It is quite flexible with respect to monitoring, inspection, and repair procedures. For the example, optimal policies were found for both inspections and repairs, and depended on the current belief state and the point in time, whereby they are not required to be stationary. Condition monitoring results were taken into account in addition to offline inspections. Furthermore, seasons were taken into account. The approach has not yet been tested for use with deterioration processes where a continuous damage size is discretized into many damage states.

\subsection{Outlook}

The optimal solution would be to develop a generic program for decision making, with build-in modules with various damage models, indicator models and procedures for monitoring, inspections, and repairs. Here, it should be possible to include all relevant information available to the decision maker. This modular approach could possibly be implemented using the approach based on Markov decision processes, if procedures are developed for use for deterioration processes. However, a system approach is often needed when making optimal decisions, and that will typically require application specific approximations. This makes it difficult to make the program totally generic, but different types of decision problems could be included. The output from the program should make it apparent to the decision maker what the recommendation is and why. In order to give the decision maker a better basis for making the right decision, information should be given for how much the expected costs for the decision alternatives differ.

The relevant question is then: Will it make a difference in the costs if advanced methods are used for decision support? How much can be won by using risk-based O\&M compared to corrective maintenance or preventive maintenance without using risk-based methods? The difference in expected costs can be calculated from a model, but the result will of course depend on the input. It is a complicated task to model real decision problems. A suitable damage model is often not available, as the design assumptions are often not fulfilled, and for condition monitoring many other things than the damage size can affect the indicator. Because the result is sensitive to the input, great care should be taken when making the model. The Bayesian approach makes it possible to use all types of information, including subjective. However, if only very subjective data is available, they could be 
far from the truth, and it might be better not to use advanced models for decision support at all. However, models that are made based on data, and therefore summarize more information than the decision maker would be able to include himself, could be the basis of a strong support tool. Decision support can be relevant for decision problems on all time scales, from days to several years. 


\section{References}

Anderson, T.L. 2005. Fracture Mechanics: Fundamentals and Applications. Third Edition. Taylor and Francis. ISBN: 0-8493-1656-1.

Andrawus, J. 2008, April. "Maintenance Optimisation for Wind Turbines." PhD thesis, The Robert Gordon University, Aberdeen.

Andrawus, J. A., J. Watson, M. Kishk, and H. Gordon. 2008. "Optimisation of wind turbine inspection intervals." Wind Engineering 32 (5): $477-490$.

Andrawus, J. A., J. F. Watson, M. Kishk, and A. Adam. 2006. "The selection of a suitable maintenance strategy for wind turbines." Wind Engineering 30 (6): 471-486.

Andrawus, J.A., J. Watson, and M. Kishk. 2007. "Modelling system failures to optimise wind turbine maintenance." Wind Engineering 31 (6): 503522 .

API. 2002. Risk-based Inspection. API Recommended Practice 580.

Benjamin, J. R., and C. A. Cornell. 1970. Probability, Statistics, and Decision for Civil Engineers. McGraw-Hill.

Bertling, L. 2002. "Reliability Centered Maintenance for Electric Power Distribution Systems." PhD thesis, Royal Institute of Technology, Stockholm.

Bertling, L., R. Allan, and R. Eriksson. 2005. "A Reliability-Centered Asset Maintenance Method for Assessing the Impact of Maintenance in Power Distribution Systems." IEEE Transactions on Power Systems 20 (1): 75-82.

Besnard, F., and L. Bertling. 2010. "An Approach for Condition-Based Maintenance Optimization Applied to Wind Turbine Blades." IEEE Transactions on Sustainable Energy 1 (2): 77-83.

Besnard, F., J. Nilsson, and L. Bertling. 2010. "On the Economic Benefits of using Condition Monitoring Systems for Maintenance Management of Wind Power Systems." IEEE 11th International Conference on Probabilistic Methods Applied to Power Systems, PMAPS 2010 , art. no. 5528992 , pp. 160-165. 
Besnard, F., M. Patriksson, A. Strömberg, A. Wojciechowski, and L. Bertling. 2009. "An Optimization Framework for Opportunistic Maintenance of Offshore Wind Power System." IEEE Bucharest Power Tech Conference.

Bharadwaj, U.R., J.B. Speck, and C.J. Ablitt. 2007. "A practical approach to risk based assessment and maintenance optimisation of offshore wind farms." This paper had been presented at 26th International Conference on Offshore Mechanics and Arctic Engineering, OMAE 2007.

Bloomstine, M.L. 2012. "Optimal maintenance of major bridges." Bridge Maintenance, Safety, Management, Resilience and Sustainability - Proceedings of the Sixth International Conference on Bridge Maintenance, Safety and Management. 2096-2103.

BS7910. 2005. Guide to methods for assessing the acceptability of flaws in metallic structures. British Standards.

Christer, A. H. 1999. "Developments in delay time analysis for modelling plant maintenance." Journal of the Operational Research Society 50 (11): 1120-1137.

Christer, A. H., and W. M. Waller. 1984. "Delay Time Models Of Industrial Inspection Maintenance Problems." Journal of the Operational Research Society 35 (5): 401-406.

Corotis, R.B., J.H. Ellis, and M. Jiang. 2005. "Modeling of risk-based inspection, maintenance and-life cycle cost with partially observable Markov decision processes." Structure and Infrastructure Engineering 1 (1): $75-84$.

Dasgupta, S., C.H. Papadimitriou, and U.V. Vazirani. 2006. Algorithms. McGraw-Hill.

Dekker, R., and P.A. Scarf. 1998. "On the impact of optimization models in maintenance decision making: the state of the art." Reliability Engineering and System Safety 60 (1998): 111-119.

DNV/Risø. 2002. Guidelines for Design of Wind Turbines. Second Edition. Det Norske Veritas and Ris $\varnothing$ National Laboratory. ISBN: 87-550-2870-5.

El-Thalji, I., and J. Liyanage. 2011. "An Asset Management Solution for Offshore Wind Farms Based on System Dynamics Simulation: A Case Study." Proceedings of ISOPE 2011.

Energiaftale. 2012, Marts. Aftale mellem regeringen (Socialdemokraterne, Det Radikale Venstre, Socialistisk Folkeparti) og Venstre, Dansk Folkeparti, Enhedslisten og Det Konservative Folkeparti om den danske energipolitik 2012-2020.

Energistyrelsen. 2012. "Energistatistik 2011: Data, tabeller, statistikker og kort." Technical Report. http://www.ens.dk/da- 
DK/Info/TalOgKort/Statistik_og_noegletal/Aarsstatistik/Documents/Energistatistik\%202011.pdf.

Engels, Wouter, Tom Obdam, and Feike Savenije. 2009. "Current developments in wind - 2009." Technical Report ECN-E-09-96, Energy research Centre of the Netherlands.

Eurocode 3-1-9. 2005. Eurocode 3: Design of steel structures - Part 1-9: Fatigue. European Committee for Standardization.

European Union. 2009. Directive 2009/28/EC of the European parliament and of the council of 23 April 2009. Official Journal of the European Union.

Faber, M. H. 2002. "Risk-based inspection: The framework." Structural Engineering International: Journal of the International Association for Bridge and Structural Engineering (IABSE) 12 (3): 186-194.

Faber, M. H., J. D. Sørensen, J. Tychsen, and D. Straub. 2005. "Field implementation of RBI for jacket structures." Journal of Offshore Mechanics and Arctic Engineering 127 (3): 220-226.

Faddoul, R., W. Raphael, and A. Chateauneuf. 2011. "A generalised partially observable Markov decision process updated by decision trees for maintenance optimisation." Structure and Infrastructure Engineering 7 (10): 783-796.

Faulstich, S., P. Hahn, and P. Tavner. 2011. "Wind turbine downtime and its importance for offshore deployment." Wind Energy 14:327-337.

Friis-Hansen, A. 2000. "Bayesian networks as a decision support tool in marine application." Ph.D. diss., Technical University of Denmark.

Gilks, W.R., S. Richardson, and D.J. Spiegelhalter. 1996. Markov Chain Monte Carlo in practice. Chapman \& Hall/CRC. ISBN: 0-412-05551-1.

Hameed, Z., Y. S. Hong, Y. M. Cho, S. H. Ahn, and C. K. Song. 2009. "Condition monitoring and fault detection of wind turbines and related algorithms: A review." Renewable and Sustainable Energy Reviews 13 (1): 1-39.

Heckerman, D. 2008. A tutorial on learning with Bayesian networks. Volume 156.

Hofmann, M., J. Heggset, L.M. Nonås, and E.E. Halvorsen-Weare. 2011. "A concept for Cost and Benefit Analysis of Offshore Wind Farms with Focus an Operation and Maintenance." Proceedings of COMADEM 2011.

Hrycej, T. 1990. "Gibbs sampling in Bayesian networks." Artificial Intelligence 46 (3): 351-363.

IEC 61400-4. 2012. Wind turbines Ü Part 4: Design requirements for wind turbine gearboxes. International Electrotechnical Commission,. 
ISO 281. 2007. Rolling bearings - Dynamic load ratings and rating life. Second edition. International Standard.

ISO 6226-2. 1996. Calculation of load capacity of spur and helical gears Part 2: Calculation of surface durability (pitting). First edition. International Standard.

ISO 6226-5. 2003. Calculation of load capacity of spur and helical gears Part 5: Strength and quality of materials. Second edition. International Standard.

ISO 6226-6. 2006. Calculation of load capacity of spur and helical gears - Part 6: Calculation of service life under variable load. First edition. International Standard.

JCSS. 2008. "Risk Assessment in Engineering - Principles, System Representation \& Risk Criteria." Technical Report, Joint Commitee on Structural Safety. ISBN 978-3-909386-78-9.

Jensen, F. V. 2008. "Approximate Representation of Optimal Strategies from Influence Diagrams." Edited by M. Jaeger and T. D. Nielsen, Proceedings of the Fourth European Workshop on Probabilistic Graphical Models. 153-159.

Jensen, F. V., and T. D. Nielsen. 2007. Bayesian Networks and Decision Graphs. Information Science and Statistics, Springer.

Kiureghian, A. D., and O. Ditlevsen. 2009. "Aleatory or epistemic? Does it matter?" Structural Safety 31 (2): 105-112.

Kovacs, A., G. Erdos, Z.J. Viharos, and L. Monostori. 2011. "A system for the detailed scheduling of wind farm maintenance." CIRP Annals Manufacturing Technology 60 (2011): 497-501.

Lauritzen, S. L., and D. Nilsson. 2001. "Representing and solving decision problems with limited information." Management Science 47 (9): 12351251.

Linneberg, P., M. Zinck, and K.V. Christensen. 2012. "Reliability based inspection and reliability centered maintenance." Bridge Maintenance, Safety, Management, Resilience and Sustainability - Proceedings of the Sixth International Conference on Bridge Maintenance, Safety and Management. 2112-2119.

Lunn, D. J., A. Thomas, N. Best, and D. Spiegelhalter. 2000. "WinBUGS - A Bayesian modelling framework: Concepts, structure, and extensibility." Statistics and Computing 10 (4): 325-337.

Madsen, H.O., S. Krenk, and N.C. Lind. 1986. Methods of Structural Safety. Prentice-Hall Englewood Cliffs, London. ISBN: 0-13-579475-7. 
Madsen, H.O., and J.D. Sørensen. 1990. "Probability-based Optimization of Fatigue Design, Inspection and Maintenance." Int. Symp. on Offshore Structures, University of Glasgow.

Madsen, H.O., J.D. Sørensen, and R. Olesen. 1989. "Optimal Inspection Planning for Fatigue Damage of Offshore Structures." ICOSSAR, San Francisco.

Madsen, H.O., A.G. Tallin, and F. Kirkemo. 1987. "Probabilistic Fatigue Crack Growth Analysis of Offshore Structures, with Reliability Updating Through Inspection." Ship Structures Symposium '87: Marine Structural Reliability Symposium.

Maes, M.A., and A. Naess. 2012. "Selecting an optimal window of opportunity within a finite horizon." International Forum on Engineering Decision Making, 6th IFED Forum.

McMillan, D., and G. W. Ault. 2007. "Quantification of Condition Monitoring Benefit for Wind Turbine Generators." Wind Engineering 31 (4): $267-285$.

McMillan, D., and G.W. Ault. 2008. "Condition monitoring benefit for onshore wind turbines: sensitivity to operational parameters." IET Renewable Power Generation 2 (1): 60-72. doi: 10.1049/iet-rpg:20070064.

Miner, M.A. 1945. "Cumulative Damage in Fatigue." J. Applied Mechanics 12:A159-A164.

Moubray, J. 2000. "The case against streamlined RCM."

Murphy, K. 2001a. An introduction to graphical models.

2002. "Dynamic Bayesian networks: Representation, inference and learning." Ph.D. diss., University of California, Berkeley.

Murphy, K.P. 2001b. "The Bayes Net Toolbox for Matlab." Computing Science and Statistics, vol. 33. Available online: http://people.cs.ubc.ca/ murphyk/Papers/bnt.pdf Downloaded 08-112012 .

Nilsson, J., and L. Bertling. 2007. "Maintenance management of wind power systems using condition monitoring systems - Life cycle cost analysis for two case studies." IEEE Transactions on Energy Conversion 22 (1): 223-229.

Nowland, F. S., and H. F. Heap. 1978, December. Reliability-Centered Maintenance. San Francisco, California: United Airlines. AD/A066 579.

Obdam, T.S., L.W.M.M. Rademakers, and H. Braam. 2009. "Flight Leader Concept for Wind Farm Load Counting." Technical Report, Energy research Centre of the Nederlands. ECN-E-09-068. 
- 2010. "Flight Leader Concept for Wind Farm Load Counting: Offshore Evaluation." Wind Engineering 34 (1): 109-122.

Paris, P. C., M. P. Gomez, and W. E. Anderson. 1961. "A rational analytic theory of fatigue." The Trend in Engineering 13:9-14.

Philips, J.L., C.A. Morgan, and J. Jacquemin. 2006. "Evaluating O\&M strategies for offshore wind farms through simulation - the impact of wave climatology." Proceedings of OWEMES 2006.

Rademakers, L.W.M.M., H. Braam, O.S. Obdam, P. Frohböse, and N. Kruse. 2008a. "Estimating costs of operation and maintenance for offshore wind farms." This paper had been presented at the EWEC 2008, ECN-M-08-027.

. 2008b. "Tools for estimating operation and maintenance costs of offshore wind farms: State of the Art." This paper had been presented at the EWEC 2008, ECN-M-08-026.

Rademakers, L.W.M.M:, H. Braam, M.B. Zaaijer, and G.J.W. van Bussel. 2003. "Assessment and optimization of operation and maintenance of offshore wind turbines." Proceedings of EWEC 2003.

Raiffa, H., and R. Schlaifer. 1961. Applied statistical decision theory. Harvard University.

Regeringen. 2011, November. Vores Energi. Energiudspil.

Russel, S., and P. Norvig. 2003. Artificial Intelligence - A Modern Approach. Second edition. Prentice Hall. ISBN: 0-13-080302-2.

Society of Automotive Engineers. 1999. Evaluation Criteria for ReliabilityCentered Maintenance (RCM) Processes. SAE JA1011.

Sørensen, J. D. 2009. "Framework for risk-based planning of operation and maintenance for offshore wind turbines." Wind Energy 12 (5): 493-506.

Stephens, R. I., A. Fatemi, R. R. Stephens, and H. O. Fuchs. 2001. Metal Fatigue in Engineering. Second edition. John Wiley \& Sons, Inc. ISBN: 978-0-471-51059-8.

Stratford, P. 2007. "Assessing the Financial Viability of Offshore Wind Farms." Proceedings of EWEC 2007.

Straub, D. 2004. "Generic approaches to risk based inspection planning for steel structures." Ph.D. diss., Swiss Federal Institute of Technology, ETH Zurich.

- 2009. "Stochastic modeling of deterioration processes through dynamic bayesian networks." Journal of Engineering Mechanics 135 (10): 1089-1099.

Straub, D., and M.H. Faber. 2004. "System effects in generic risk-based inspection planning." Journal of Offshore Mechanics and Arctic Engineering 126 (3): 265-271. 
—. 2005. "Risk based inspection planning for structural systems." Structural Safety 27 (4): 335-355.

_. 2006. "Computational aspects of risk-based inspection planning." Computer-Aided Civil and Infrastructure Engineering 21 (3): 179-192.

Tian, Z., T. Jin, B. Wu, and F. Ding. 2011. "Condition based maintenance optimization for wind power generation systems under continuous monitoring." Renewable Energy 36 (2011): 1502-1509.

van Bussel, G.J.W., and W.A.A.M. Bierbooms. 2003. "The DOWEC Offshore Reference Windfarm: analysis of transportation for operation and maintenance." Wind Engineering 27 (5): 381-392.

Walford, C. A. 2006. "Wind Turbine Reliability: Understanding and Minimizing Wind Turbine Operation and Maintenance Costs." Technical Report SAND2006-1100.

Wang, H. 2002. "A survey of maintenance policies of deteriorating systems." European Journal of Operational Research 139 (2002): 469-489.

Welte, T.M., and A.O. Eggen. 2008. "Estimation of Sojourn Time Distribution Parameters Based on Expert Opinion and Condition Monitoring Data." Proceedings of the 10th International Conference on Probabilistic Methods Applied to Power Systems: 1-8.

Wiggelinkhuizen, E., T. Verbruggen, H. Braam, L. Rademakers, J. Xiang, and S. Watson. 2008. "Assessment of condition monitoring techniques for offshore wind farms." Journal of Solar Energy Engineering, Transactions of the ASME 130 (3): 0310041-0310049.

Wiggelinkhuizen, E., S. Watson, G. Giebel, E. J. Norton, M. C. Tipluica, A. J. Christensen, and E. Becker. 2007. "Conmow Final Report." Technical Report ECN-E-07-044, Energy research Centre of the Nederlands. 



\section{Appendix A}

\section{Papers}

This appendix contains the following papers:

Paper 1: Nielsen, J. J. and J. D. Sørensen. 2011. "On Risk-Based Operation and Maintenance of Offshore Wind Turbine Components." Reliability Engineering \& System Safety 96 (2011): 218-229. DOI: 10.1016/j.ress.2010.07.007.

Paper 2: Nielsen, J. J. and J. D. Sørensen. 2010. "Planning of O\&M for Offshore Wind Turbines using Bayesian Graphical Models." ESREL 2010 annual conference, Rhodes, Greece.

Paper 3: Nielsen, J. J. and J. D. Sørensen. 2011. "Risk-Based Operation and Maintenance of Offshore Wind Turbines using Bayesian Networks." 11th International Conference on Applications of Statistics and Probability in Civil Engineering, ICASP11, ETH, Zurich, Switzerland.

Paper 4: Nielsen, J. J. and J. D. Sørensen. 2011. "Challenges for Riskbased Maintenance Planning for Offshore Wind Turbines." 21st International Offshore and Polar Engineering Conference, ISOPE 2011, Maui, Hawaii, USA.

Paper 5: Nielsen, J. S. and J. D. Sørensen. 2012. "Maintenance Optimization for Offshore Wind Turbines using POMDP." IFIP WG 7.5 Working Conference on Reliability and Optimization of Structural Systems, AUA, Yerevan, Armenia.

Paper 6: Nielsen, J. S., R. P. v.d. Pieterman and J. D. Sørensen. 2013. "Analysis of pitch system data for condition monitoring." Wind Energy (Published online). DOI: 10.1002/we.1586. 



\section{A.1 Paper 1}

\section{Title:}

\section{On Risk-Based Operation and Maintenance of Offshore Wind Turbine Components}

Authors:

Nielsen, J. J. and J. D. Sørensen.

Published in:

Reliability Engineering \& System Safety 96 (2011): 218-229. DOI: 10.1016/j.ress.2010.07.007. 



\title{
On risk-based operation and maintenance of offshore wind turbine components
}

\author{
Jannie Jessen Nielsen ${ }^{\text {a,* }}$, John Dalsgaard Sørensen ${ }^{\text {a,b }}$ \\ ${ }^{a}$ Department of Civil Engineering, Aalborg University, Sohngaardsholmsvej 57, DK-9000 Aalborg, Denmark \\ ${ }^{\mathrm{b}}$ Wind Energy Division, Risø-DTU, Denmark
}

\section{A R T I C L E I N F O}

\section{Article history:}

Received 2 January 2010

Received in revised form

13 July 2010

Accepted 16 July 2010

Available online 21 July 2010

Keywords:

Offshore wind turbine

Operation and maintenance

Risk-based

Life-cycle decision analysis

Simulation

\begin{abstract}
A B S T R A C T
Operation and maintenance are significant contributors to the cost of energy for offshore wind turbines. Optimal planning could rationally be based on Bayesian pre-posterior decision theory, and all costs through the lifetime of the structures should be included. This paper contains a study of a generic case where the costs are evaluated for a single wind turbine with a single component. Costs due to inspections, repairs, and lost production are included in the model. The costs are compared for two distinct maintenance strategies, namely with and without inclusion of periodic imperfect inspections. Finally the influence of different important parameters, e.g. failure rate, reliability of inspections, inspection interval, and decision rule for repairs, is evaluated.
\end{abstract}

(c) 2010 Elsevier Ltd. All rights reserved.

\section{Introduction}

The costs of operation and maintenance (O\&M) of offshore wind turbines are significant contributors to the cost of energy - up to $30 \%$ of the cost of energy. Optimal planning of O\&M should include the use of inspections and monitoring results to make decisions that minimize the expected total costs through the lifetime of the structures. For offshore wind turbines it is especially important because of the dependence on weather windows for inspections and repairs to be possible. The aim of this work is to demonstrate the effect of the use of conditionbased maintenance compared to the use of corrective maintenance for a generic case. For simplicity, only a single wind turbine with a single component is included in model, but the relative influence of different parameters are considered to be generic.

\subsection{Maintenance activities}

In general maintenance activities can be divided into corrective and preventive maintenance. Corrective maintenance is performed if a component has failed, and preventive maintenance is performed to avoid failure. Preventive maintenance can be divided into scheduled and condition-based maintenance. Scheduled maintenance is performed on scheduled times, and could e.g.

\footnotetext{
* Corresponding author. Tel.: +4599408575.

E-mail address: jjn@civil.aau.dk (J.J. Nielsen).
}

be lubrication, tightening bolts, and changing filters [1]. Condition-based maintenance is performed on the basis of the actual health of the component, and thus it requires a conditionmonitoring system with online monitoring and/or inspections, see e.g. [2,3]. For offshore wind turbines service visits are performed on a scheduled basis, where scheduled maintenance are performed, and at the same time inspections can be performed at a relatively low additional cost.

The use of corrective maintenance is the most simple strategy, but it has several flaws. The failure of one minor component can cause escalated damage to a major component, which gives large repair/replacement costs. Further failures will often happen during a period with large wind loads, and the site will be unaccessible during that period, which will cause lost production, see e.g. [4]. Thus the costs for corrective maintenance are associated with much larger uncertainty than preventive maintenance [2].

\section{Optimal planning of inspection and maintenance}

Optimal planning of O\&M should basically be based on riskbased methods, where pre-posterior decision theory is used to take all available information from past experience, inspections, and monitoring into account. The theoretical basis is described in this section and is based on the wind turbine framework [5] and general theory in [6]. 


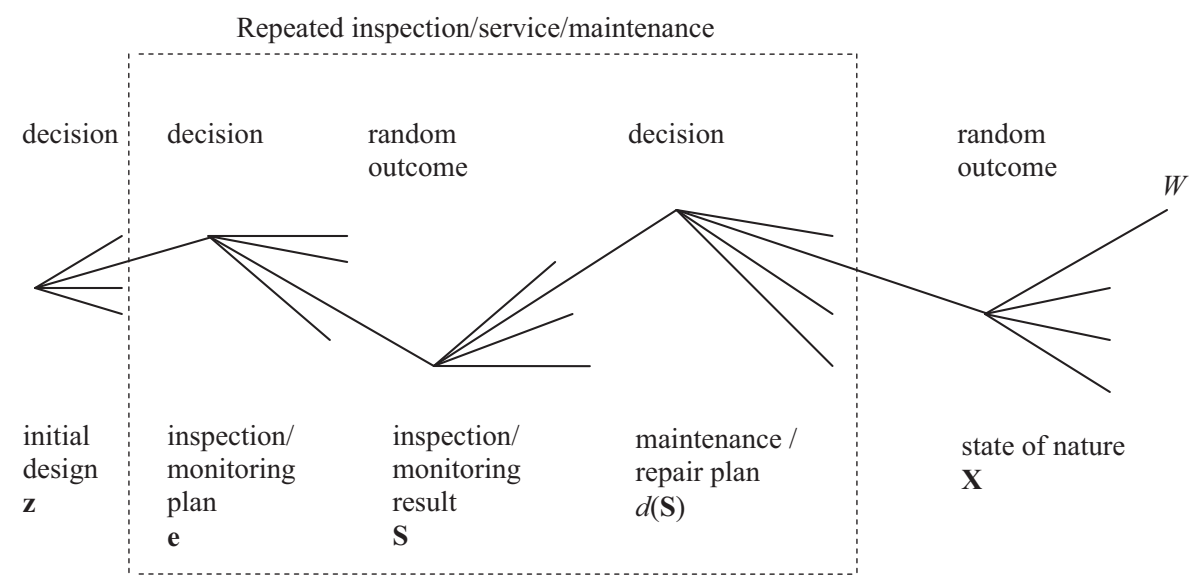

Fig. 1. Decision tree for optimal planning of inspections and maintenance [5].

The problem of making decisions that minimize the expected costs through the lifetime of the wind turbines can be represented using a decision tree as shown in Fig. 1. In this model there are three types of decisions: initial decisions regarding the design, decisions regarding the inspections/monitoring and decisions on maintenance/repair.

The initial design decisions, $z$, are included in the model, because the design will influence the realizations of the damage, and thereby the inspection, maintenance and repair costs. In principle there are an infinite number of possible decisions, but this has to be narrowed down to a number of specific designs, in order to make an analysis. Relevant parameters at the initial design stage could be whether to install a permanent crane in the nacelle, and the design life of different fatigue, corrosion, and wear exposed components. Application of risk-based decision models requires that the condition of the wind turbine components/systems can be described by damage models.

The central decision in the analysis is the decision on when and how to maintain and perform repair. Lack of repair before component failure might lead to loss of production and extra costs due to escalated damage. If, on the other hand, a repair is performed before it is necessary, the total number of repairs during the lifetime will be larger than necessary. If a perfect damage model was available it would be straightforward to make the optimal decision; to repair just in time. However, this is not the case because the damage model is associated with uncertainties, and the state of nature, $\mathbf{X}$, of the components are unknown, e.g. stochastic variables describing fatigue development with time.

The prior probability model of the state of nature is denoted $P_{X}$. In order to obtain further information on the state of nature, and thereby decrease these uncertainties, experiments, $e$, can be made, at some cost. For an offshore wind turbine these "experiments" can be divided into online condition-monitoring and manual inspections, see e.g. [7]. Depending on the component of interest different methods are available, and in general these methods will be imperfect in the sense, that the outcome of the investigation, $\mathbf{S}$, can be coupled with the state of nature, $\mathbf{X}$, but with some uncertainty that can be quantified through the conditional probability $P_{S \mid X}$.

With the new information gained from the monitoring/ inspections, Bayesian updating can then be used to determine the posterior probability $P_{X \mid S}$ for the state of nature. On the basis of this updated probability a decision, $a$, regarding maintenance/ repair must be made. This decision cannot be made before the result from the inspection/monitoring is made, but instead a decision rule, $d(\mathbf{S})$, can be determined/chosen at the design stage as a function of the outcome of the inspection/monitoring, $\mathbf{S}$. This rule states what decision to make, for all possible outcomes of the inspection/monitoring.

The basis for the calculations also includes the utility corresponding to each branch of the tree, in this case quantified in monetary value. The job to find the decisions that maximize the expected utility, that is the expected total gain minus the expected costs, can be done in two different ways, normal or extensive form. In the extensive form analysis the starting point is in the final branches of the tree at the right in Fig. 1. For each branch after the final decision the expected utility is calculated, and for each possible outcome of the experiment, the maximum expected utility is found. The same is done for all branches after the decision on which experiment to make, and the maximum value of the expected utilities gives the optimal choice of inspection/monitoring. In the normal form analysis every possible decision rule for a given experiment is explicitly considered, and for each experiment an optimal decision rule is found. The maximum expected utilities for each available experiment for normal and extensive form, respectively, are given by

$u^{*}(e)=\max _{d} E_{X} E_{S \mid X} u(e, \mathbf{S}, d(\mathbf{S}), \mathbf{X})$

$u^{*}(e)=E_{S} \max _{a} E_{X \mid S} u(e, \mathbf{S}, a, \mathbf{X})$

$u^{*}$ is maximum utility and $E$ denotes expectation with respect to the subscripted variables. Because $E_{X} E_{S \mid X}$ is the expectation over $\mathbf{X} \times \mathbf{S}$ it equals $E_{S} E_{X \mid S}$, and it can be shown that the two expressions give same utility for each experiment $e$. The optimal decision $e^{*}$ is the one maximizing $u^{*}(e)$ i.e. $\max _{e} u^{*}(e)$.

In principle either analysis forms can be used. In this analysis the expectation is evaluated using simulation, and thus the normal form gives the most direct approach, as $E_{X} E_{S \mid X}=E_{X S}$ is the expectation with respect to $\mathbf{X}$ and $\mathbf{S}$, and can be evaluated in one step. In the extensive form the simulations should be divided into two steps in order to maximize with respect to the decision rule before the state of nature is found.

Based on the normal form analysis the optimization problem is to maximize the total gain minus the costs, $W$, and it can be formulated as

$\max _{z, e, d} W=B-C_{I}-C_{I N}-C_{R E P}-C_{F}$

where $B$ is the expected benefits, $C_{I}$ is the initial costs, $C_{I N}$ is the inspection and service costs, $C_{R E P}$ is the expected repair and maintenance costs and $C_{F}$ is the expected failure costs, and all values are functions of the design parameters, $\mathbf{z}$, inspections parameters, e, and decision rules for repairs, $d$. 
Eq. (3) should optimally be applied to an entire wind farm, and the costs for all components should be accounted for. For simplicity the equation is applied to just one component in one wind turbine in the application example. It should be noted that the costs for all components and all wind turbines cannot just be added in order to find the global optimal strategy, as many other aspects should be taken into account. Extension of the model to a wind farm and wind turbines with many components is discussed in Section 8.

\section{Model description}

This section describes the generic model that has been made to model the operation and maintenance costs for a single wind turbine with a single component. The damage level of the component increases over time, and if it exceeds the damage level equal to 1 the wind turbine stops producing power. After the component is repaired it starts producing power again.

In the model both corrective and condition-based maintenance is included, and the condition-monitoring system consists of scheduled inspections performed at the same time as routine service.

For corrective maintenance there are costs due to lost power production and repairs. If condition-based maintenance is enabled scheduled inspections are performed, and hence it is possible to detect a damage before it causes the wind turbine to stop producing power. This comes with an additional cost due to the inspections, but some of the costs due to lost power production can be saved.

Online monitoring could be included in the model as well, and thus beginning defects could be detected while the wind turbine is still producing power, and some failures/corrective repairs could be avoided. This will give less lost production due to failures, but might also give more false detections because the online monitoring is less certain than inspections. Further, indirect information from online monitoring will often be associated with additional uncertainty.

The weather influences several aspects of the model. The power production depends on the wind speed, and the damage accumulation depends on the significant wave height and wind speed. For inspections and repairs to be performed by boat, it is necessary that both wind speed and significant wave height are below a certain limit. Alternatively repairs can be performed by helicopter that only has a non-strict limit concerning the wind speed. It is more expensive to use a helicopter than a boat, but lost production can be decreased.

\section{Parameters}

The model generates the costs of inspections, repairs and lost production over the lifetime of a wind turbine. The randomness of the nature is modeled by the use of a number of random variables as explained in this section.

\subsection{Weather}

The weather data applied in the example below are from the wind farm Horns Rev 2 in Denmark. The mean wind speed and mean significant wave height for every hour have been provided by DHI (Danish Hydraulic Institute) for years 1979-2007.

Pseudo-random weather is generated for the lifetime of the wind turbine, 20 years, by bootstrapping the real data on a yearly basis. This method gives a randomness in the weather, without compromising the validity. The realizations of the weather are included in the state of nature $\mathbf{X}$ in Fig. 1.

\subsection{Power production}

The power production depends on the wind speed, and is calculated for $1 \mathrm{~h}$ intervals. A power curve for a $5 \mathrm{MW}$ wind turbine is used and is shown in Fig. 2.

The power price is assumed to increase with the rate of inflation, and the initial power price is set to a constant $b$. In reality there will be fluctuations in the power price, but the influence of these is assumed to be negligible and is omitted in the analysis.

\subsection{Damage}

The damage size of the component is measured on a relative scale where 0 means that no damage is present, and 1 means failure causing the wind turbine to stop producing power because of the damage. Damage initiations is not considered in the model, thus it is assumed that an initial damage is present for a new component as well as after a repair.

It is only meaningful to use condition-based maintenance for components where the damage can be detected before failure occurs. In general damage accumulation for such components can be described by an initial value problem, with the following differential equation and initial damage size $D(0)=D_{0}$ :

$\frac{d D}{d t}=C \cdot F^{m_{1}} \cdot D^{m_{2}}$

where $d D / d t$ is the rate of damage growth, $F$ is a measure of the load, $D$ is the damage size, $C, m_{1}$, and $m_{2}$ are model parameters. The load variable can be a function of e.g. wind speed and wave height. If a specific component is considered, the wind turbine will act as a filter which the loads from waves and wind pass through, and the correlation between them will change due to the influence of the control system.

A numerical solution to the initial value problem can be found using the Euler method, where the damage size at each time step is calculated by

$D_{t+\Delta t}=D_{t}+\frac{d D}{d t} \cdot \Delta t$

In this model a time step $\Delta t$ of $3 \mathrm{~h}$ is used.

The damage level after a repair will depend on the repair method used and how well it has been performed, which can also be influenced by the number of repairs performed. For simplicity the damage level after a repair is here assumed to be independent

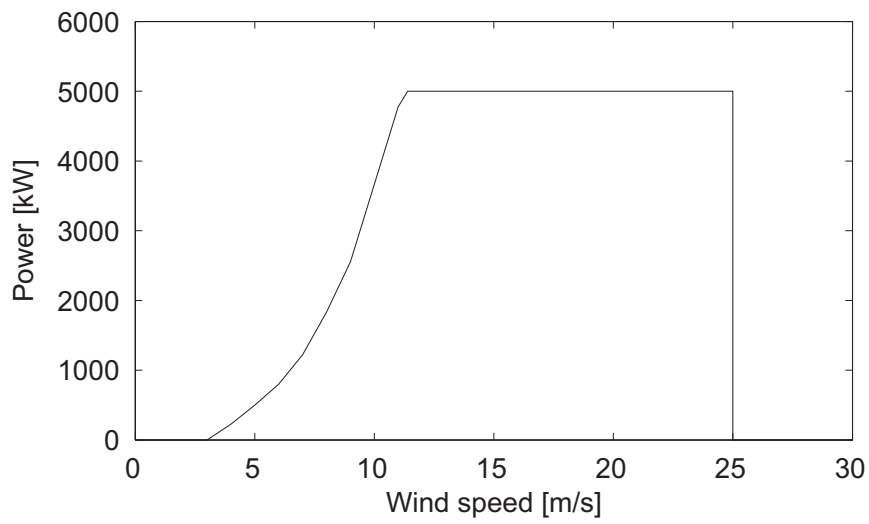

Fig. 2. Power curve for $5 \mathrm{MW}$ wind turbine [8]. 
of the history of the component, and it is modeled by a stochastic variable $D_{0}$.

The outcomes of the random variables are included in the state of nature $\mathbf{X}$ in Fig. 1.

\subsection{Inspections}

Inspections are scheduled to be performed at the service visits every 180 days. However, if it is found that the weather conditions that day do not allow for a boat to be used, the inspection is moved to the first coming day with acceptable weather after the scheduled date. The influence of the inspection interval on the costs is investigated in Section 7.3.

In general there is a probability that a present damage is not detected at an inspection, and in addition there is a probability that there is an indication of damage, without the damage being present. The reliability of the inspection depends on the inspection method, and in general it can be chosen to make a more reliable inspection that on the other hand is more expensive. In the present calculations the probability of false indications is not taken into account, and the probability of detection $(P o D)$ of the damage is modeled with the PoD curve:

$\operatorname{PoD}(D)=P_{0}\left(1-\exp \left(\frac{-D}{\lambda}\right)\right)$

$D$ is the damage level, $P_{0}$ is the maximum probability of detection, and $\lambda$ is the expected value of smallest detectable damage as described in e.g. [9]. The probability of false indications (PFI) could be taken into account by the probability of indication (PoI), given by

$\operatorname{PoI}(D)=\operatorname{PoD}(D)+(1-P o D(D)) P F I$

If the damage is detected at the inspections, the correct size of the damage is assumed to be measured by the inspection. A measurement uncertainty could be included in the model by adding a measurement error to the real value of the damage, $D$ :

$D_{\text {ins }}=D+\varepsilon$

where $\varepsilon$ is a normal distributed random variable with mean zero, and $D_{i n s}$ is the damage level found by an inspection. For simplicity the measurement error is in this example assumed to be zero.

The values of the inspection parameters are in the example below set to $\lambda=0.4$ and $P_{0}=1$, which gives the $P o D$-curve shown in Fig. 3. These values will be influenced by the inspection procedure, on which nothing in particular is assumed here. However, a sensitivity study is applied to assess the influence of the assumption in Section 7.2.

The cost of one inspection is set to $C_{I N S P}$.

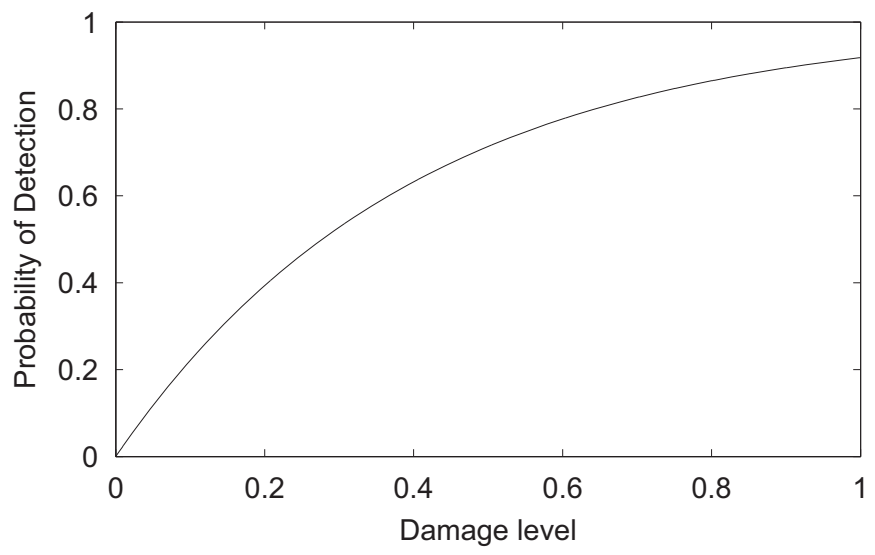

Fig. 3. Probability of detection as a function of damage level.
The parameter $\lambda$ and the inspection interval represent the inspection plan, $e$, in Fig. 1 . The inspection result $D_{\text {ins }}$ equals the inspection result $\mathbf{S}$.

\subsection{Repairs}

Repairs are separated into condition-based and corrective repairs. Condition-based repairs are executed on the same day as the inspection, if the detected damage is larger than a limit value $D_{\text {rep. }}$. The value of $D_{\text {rep }}$ should be chosen significantly lower than the value that causes failure, in order to make it unlikely that the failure will occur before next inspection. Optimally the value should be found such that the expected total costs are minimized, as shown in the example in Section 7.1.

A corrective repair is ordered, if the damage reaches $D \geq 1$, which means that a failure has occurred. The number of days necessary to perform a corrective repair is assumed lognormal distributed. In the example below a mean $\mu=3$ days and coefficient of variation $\mathrm{COV}=0.5$ are used, but it can only take integer values, and has a minimum of one day. Mobilization of the crew is assumed to take the rest of the day when the damage exceeds 1 .

The price for a repair is assumed to depend on the transport type, if the damage level has exceeded 1 , and the number of days necessary for a repair, as described in Table 1.

As explained above the decision rule, $d(\mathbf{S})$, is modeled by the rule: repair if $D_{\text {ins }} \geq D_{\text {rep }}$, where $D_{\text {rep }}$ is the repair limit value, and $D_{i n s}$ is the inspection result. $D_{\text {ins }}$ is equal to the damage size $D$ if a damage is detected, else it is zero.

\subsection{Transport}

Three transport options for corrective repairs are implemented:

Option 1: Always use a boat for repairs.

Option 2: Repair as soon as possible (ASAP). A boat is used if there are sufficient good weather conditions starting the day after the damage level has exceeded 1 , else the helicopter is used.

Option 3: Risk-based alternative, where the cheapest solution is calculated given a perfect weather forecast. The first coming days where repair is possible by boat or helicopter, respectively, are found, and the cost of the repair and lost production until that day is found for each transport type. The assumption that a perfect weather forecast is available until both repairs are possible will not be the case in reality, but it gives the lower limit for the costs for a case with these two transport options.

For all three options it is assumed that a perfect weather forecast is available for the duration of the repair. This means that a repair that has been started will never be interrupted by bad weather.

Table 1

Cost of repairs.

\begin{tabular}{lll}
\hline Damage & Transport & Cost (euro) \\
\hline$D<1$ & Boat & $C_{R}$ \\
$D \geq 1$ & Boat & $C_{R}\left(C_{B 1}+C_{B 2} \cdot\right.$ repair days $)$ \\
$D \geq 1$ & Heli & $C_{R}\left(C_{H 1}+C_{H 2} \cdot\right.$ repair days $)$ \\
\hline
\end{tabular}


A fourth option could be to implement a more realistic situation, where the weather forecast is uncertain, e.g. based on the forecasting methods described in e.g. $[10,11]$. There would then be a probability that the weather turns bad during an inspection/repair, and this would lead to additional costs. A decision rule should be included on when to take the risk of performing an inspection/repair, and the optimal decision rule could be found using risk based methods similar to the procedure in this paper. For simplicity this option has not been implemented in the present paper.

The boat is assumed to require a mean wind speed, $w$, less than $10 \mathrm{~m} / \mathrm{s}$ and a significant wave height, $H_{s}$, less than $1.5 \mathrm{~m}$. The used values are daily maximum values of the $1 \mathrm{~h}$ mean values. The helicopter is assumed to be able to operate at wind speeds less than $20 \mathrm{~m} / \mathrm{s}$. These values will depend on the vessels available, and are here chosen based on [12].

\subsection{Rate of interest}

All costs are discounted into present values, $C_{0}$, using the formula:

$C_{0}=\frac{C}{(1+r)^{T}}$

where $C$ is the real cost, $T$ is the time in years where the costs occurs, and $r$ is the real rate of interest, given as the nominal interest rate minus the inflation rate.

\section{Implementation of model}

The model includes the following repair- and transportation strategies, described above:

- Repair strategy:

1. Only corrective repairs.

2. Both condition-based and corrective repairs.

Transportation strategy:

- 1. Boat.

2. ASAP.

3. 'Risk' with perfect weather forecast.

For each realization of the uncertain parameters the costs throughout the lifetime of the wind turbine is calculated. The following steps are followed:

- Weather is generated.

- Weather conditions sufficient for boat and helicopter are found.

- Service/inspection times are set.

- Initial damage parameters and damage level are found.

For each day the following steps are followed:

- Possible power production is found (for $1 \mathrm{~h}$ intervals).

- Damage accumulation is found (for $3 \mathrm{~h}$ intervals).

- If an inspection is scheduled: Observable damage level is found using the PoD curve.

A repair is ordered same day, if $D_{\text {ins }} \geq D_{\text {rep }}$.

The inspection costs are found.

If repair is ordered the specific day:

- Initial damage parameters and damage level are set. The repair costs are found.

If no repair is ordered, but $D \geq 1$ :

- $\quad$ Number of days necessary for repair is found. Sufficient weather window for repair is found, and repair is scheduled.
Finally the lost production is found as the possible production the days where $D \geq 1$. All costs are discounted into present values and summed over the lifetime.

\section{Application example}

In this section results are shown for the use of different strategies for a component with a specific damage model. The considered component is assumed to have a failure rate of $0.5 /$ year. Most components will in reality have a smaller failure rate than this, see e.g. [13]. However, this example aims at investigating the influence of different parameters, and this is more clearly shown for a larger failure rate, and convergence is reached faster.

The damage accumulation is modeled by an exponential damage model which e.g. is appropriate for fatigue driven damage. The differential equation is in this case given by Paris law, which is described in e.g. [9]. For illustration of the model it is assumed that the damage accumulation is driven by the wave loads, even though wind will be dominating for most wind turbine components. The number of cycles per hour is assumed to be constant $d N / d t=360 / \mathrm{h}$, and the rate of damage accumulation is now given as

$\frac{d D}{d t}=\frac{d N}{d t} \cdot C \cdot \Delta K^{m}$

where $C$ is the damage coefficient and $m$ is the damage exponent. $\Delta K$ is the change in damage intensity factor, which depends on the current damage size $D$ and is given by

$\Delta K=\beta \cdot \Delta s \cdot \sqrt{\pi D}$

$\beta$ is the geometry factor and $\Delta s$ is the cyclic damage range that is here considered to be proportional to the mean significant wave height $H_{s}$ in the $3 \mathrm{~h}$ period:

$\Delta s=H_{s} \cdot x_{s}$

$x_{s}$ is the proportionality factor which also models the uncertainty in estimating $\Delta s$. The wind speed could also influence the deterioration, but this is indirectly taken into account, as the significant wave height and wind speed are correlated.

The damage parameters are in the example assumed to follow the values and distributions as shown in Table 2, where the significant wave height, $H_{s}$, in the damage model should be inserted in meters. The mean values are calibrated to a failure rate of $0.5 /$ year. The specific costs are set to the values shown in Table 3.

\subsection{Corrective repairs}

Fig. 4 shows the damage development for a single simulation with only corrective repairs and the risk-based transport strategy. The damage starts with an initial value and increases according to the damage function. When it reaches a value of one there are no production for some days until the component is repaired, and the damage again is set to an initial value.

Table 2

Means, coefficients of variation, and distribution types for damage parameters.

\begin{tabular}{llll}
\hline Symbol & Mean & COV & Distribution \\
\hline$m$ & 2 & - & Deterministic \\
$\beta$ & 1 & - & Deterministic \\
$C$ & $9.26 \times 10^{-10}$ & 0.2 & Lognormal \\
$x_{s}$ & 11.5 & 0.1 & Lognormal \\
$D_{0}$ & 0.02 & 0.02 & Exponential \\
\hline
\end{tabular}


In Fig. 5 the effect of the use of the different transport strategies can be seen. When ASAP is used, most repairs are performed by helicopter, whereas most repairs are performed by boat in the risk-based alternative. Of course the distribution depends on the assumptions regarding costs, limits for the weather, etc.

The costs due to lost production are large when only a boat is used, and thus the total costs are large. For the ASAP strategy the main contributor to the total cost is repairs by helicopter. The total costs are smallest for the risk-based strategy, as it should be, because the decision rule is always to choose the cheapest alternative, given a perfect weather forecast.

\subsection{Condition-based repairs}

Fig. 6 shows the damage development for a simulation with both corrective and condition-based repairs, with a value of the

Table 3

Specific costs.

\begin{tabular}{lll}
\hline Description & Symbol & Value \\
\hline Power price & $b$ & 0.04 euro/kWh \\
Inspection cost & $C_{I N S P}$ & 2500 euro \\
Repair cost & $C_{R}$ & 10000 euro \\
Boat cost factors & $C_{B} 1 / C_{B} 2$ & $1.0 / 0.5$ \\
Helicopter cost factors & $C_{H 1} / C_{H 2}$ & $1.5 / 0.75$ \\
Real rate of interest & $r$ & $5 \%$ \\
\hline
\end{tabular}

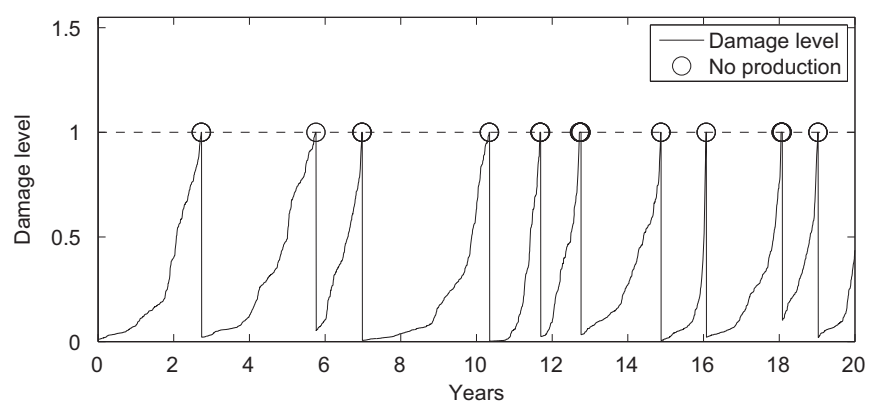

Fig. 4. Simulation of damage development for corrective maintenance with riskbased transport strategy. repair limit of the damage of $D_{\text {rep }}=0.2$. The risk-based transport strategy is used for corrective repairs. The use of condition-based maintenance means that some repairs are performed before the wind turbine actually fails. The undetected damages in Fig. 6 mean that an inspection has been performed, but that the damage is not detected.

Fig. 7 shows the effect of the use of condition-based maintenance, compared to only corrective maintenance. The number of repairs are larger for the condition-based maintenance, and most of the repairs are performed at inspections. The inspections give an additional cost, but the repairs by the inspections are sufficiently cheap compared to corrective repairs, so the total costs due to condition-based maintenance is smaller than to corrective.

\section{Parameter study}

In this section the influence of different parameters is investigated.

\subsection{Influence of $D_{\text {rep }}$}

Fig. 8 shows the influence of the limit value of the damage level for a condition-based repair to be executed, $D_{\text {rep }}$. The total number of repairs are larger for condition-based maintenance

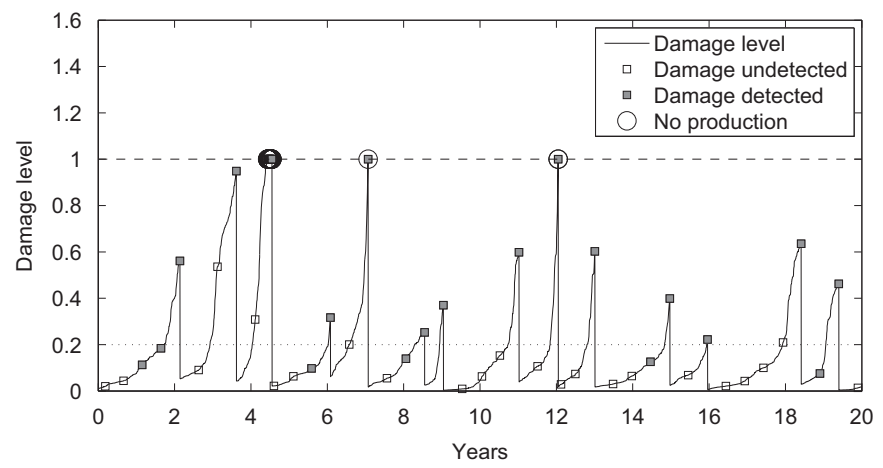

Fig. 6. Damage development for condition-based maintenance with risk-based transport strategy.

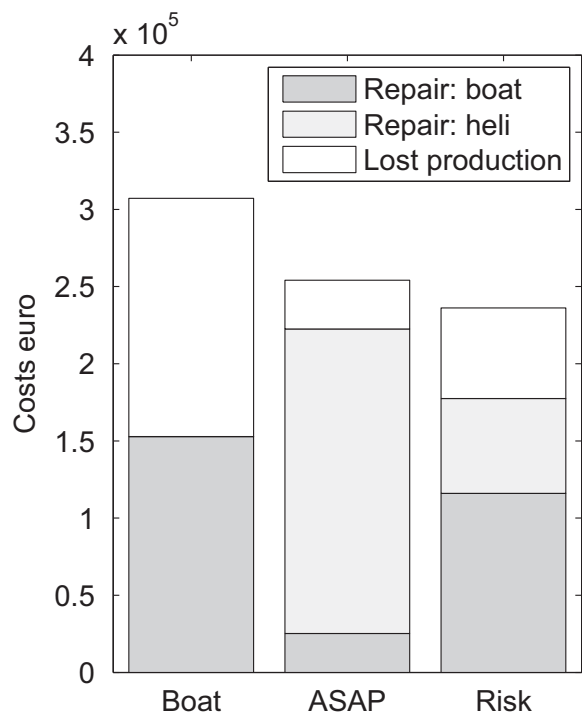

Fig. 5. Influence of transport strategy. 

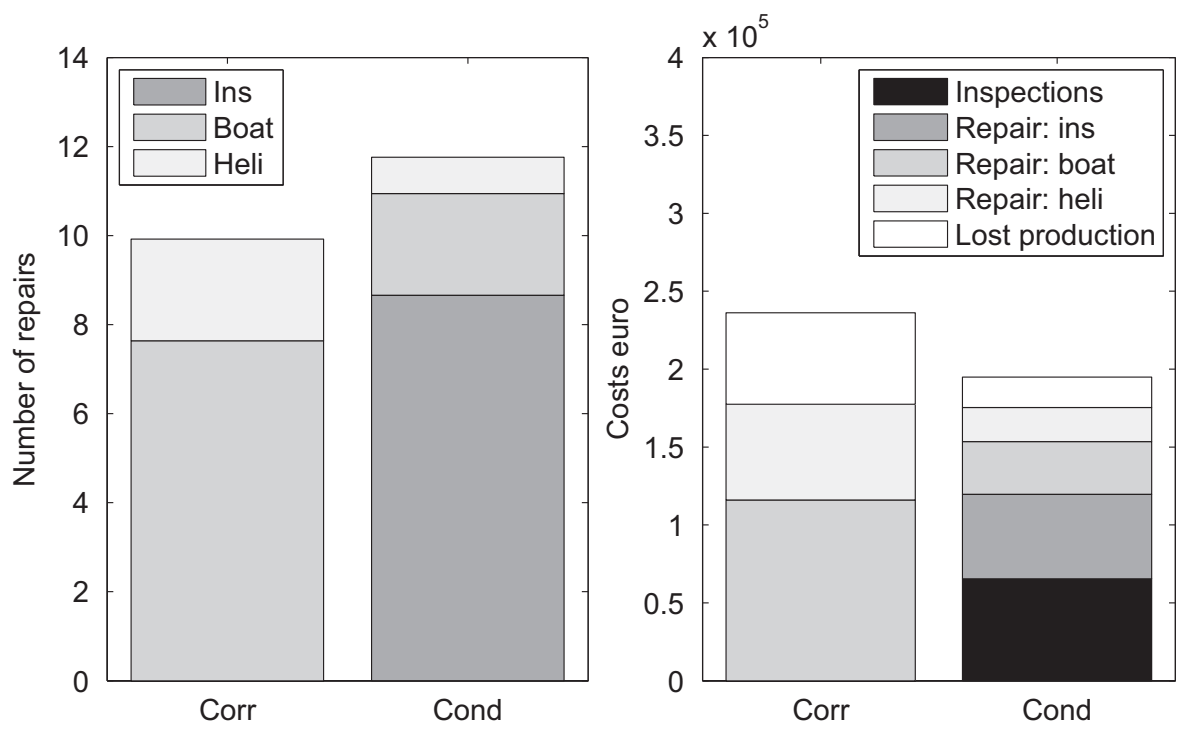

Fig. 7. Influence of condition-based maintenance. 'Corr' means corrective repairs and 'Cond' means condition-based repairs.
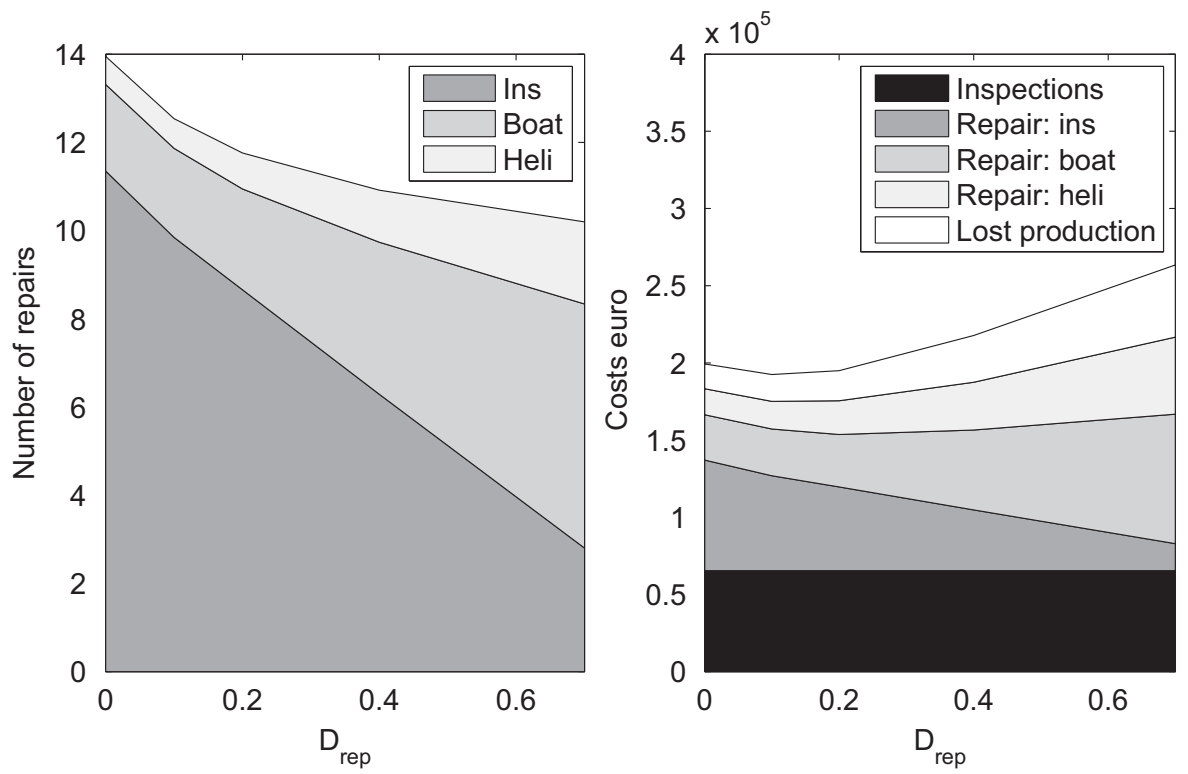

Fig. 8. Influence of $D_{\text {rep }}$.

with low $D_{\text {rep }}$, because the repairs are performed before it is actually necessary. On the other hand the number of corrective repairs by boat or helicopter is lower. Even though more repairs are performed when a low value of $D_{\text {rep }}$ is used, the total costs are found to be smallest at $D_{\text {rep }}=0.1$, because repairs at inspections are cheaper than corrective repairs. For a limit of $D_{\text {rep }}=0$ corresponding to that repairs are performed whenever a damage is detected, more repairs are performed at inspections, but it does not decrease the number of corrective repairs, and thus the total costs increase. It should be noted that the optimal value of $D_{\text {rep }}$ will be influenced by the relative values of the different costs.

\subsection{Influence of $\lambda$}

The influence of the expected value of detectable damage $\lambda$, can be seen in Fig. 9. A small value means that it is easier to detect damages, and thus more repairs are executed at inspections. This gives a decrease in the total costs because less corrective repairs are necessary. The value of $\lambda$ depends on the inspection method, and the cost of the inspections with a smaller value of $\lambda$ will be more expensive, and this is not taken into account in the shown costs. From the costs shown in Fig. 9 it can be found that if $\lambda$ can be decreased by 0.1 , then an inspection can be allowed to be up to $15.5 \%$ more expensive.

\subsection{Influence of inspection/service interval}

The influence of the inspection interval can be seen in Fig. 10. For a short inspection interval more condition-based repairs are performed, and the costs due to inspections are dominating. At an inspection interval of 6 months the total costs have a minimum. At higher inspection intervals the costs due to corrective repairs increase more than the costs due to inspections decrease. It should be noted that the time of the inspection might influence the results due to different seasons. 

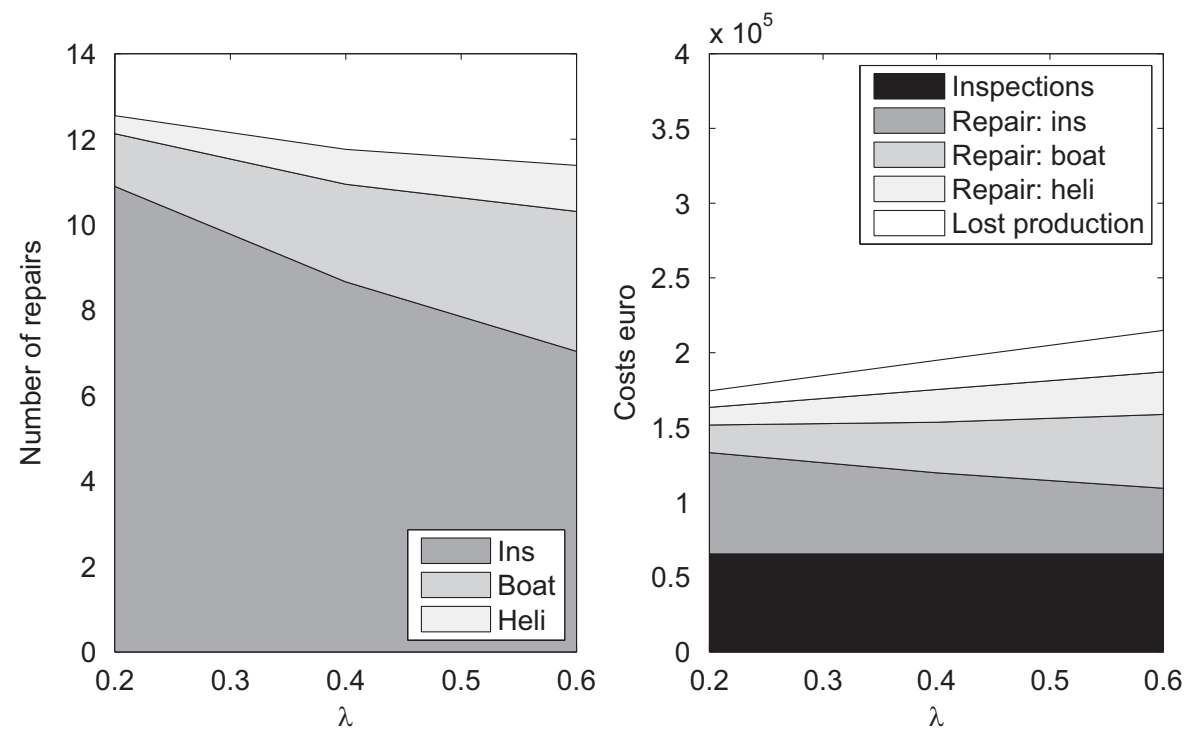

Fig. 9. Influence of $\lambda$.
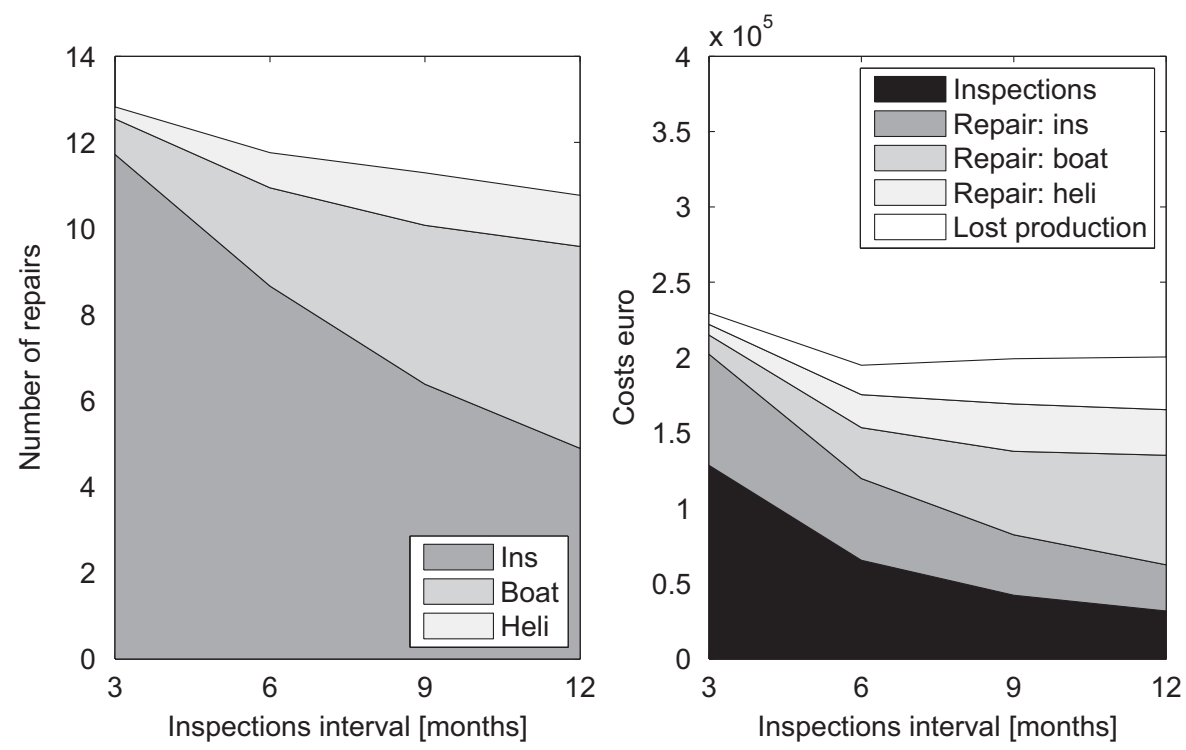

Fig. 10. Influence of inspection interval.

\subsection{Influence of real rate of interest}

In Fig. 11 the influence of the real rate of interest can be seen for corrective and condition-based repairs. In general the costs discounted into present values are smaller for a larger real rate. However, it is interesting to investigate the effect of the real rate on the costs due to condition-based maintenance relative to corrective. To the right in Fig. 11 the costs are normalized with respect to the total costs for corrective maintenance for a real rate of $5 \%$ and $10 \%$, respectively. The relative costs due to conditionbased maintenance are almost equal in the two cases, but there seems to be a minor increase at a real rate of $10 \%$. This is expected, as costs are in general paid earlier at condition-based maintenance.

\subsection{Influence of damage exponent $m$}

The damage exponent $m$ influences the acceleration of the damage growth. The larger the value, the more sudden will the damage increase from a very low value to 1 , and thus it becomes more difficult to detect a damage at inspections. In this analysis the failure rate is held constant at $0.5 /$ year, so when $m$ is changed, $x_{s}$ is calibrated to give the same failure rate. ${ }^{1}$

The influence of the damage exponent $m$ can be seen in Fig. 12 . It has no influence on the case with corrective maintenance, as only the failure rate has an influence here, but the costs due to condition-based maintenance increases with larger $m$-value, because less repairs are done at the inspections, and thus more corrective repairs have to be done. At $m=2.6$ it can be seen that the costs caused by inspections are as expensive than the savings due to less corrective repairs.

\subsection{Influence of failure rate}

The failure rate has a large influence on the costs of O\&M. If the mean time between failures (MTBF) is increased, the costs will

\footnotetext{
${ }^{1}$ Calibrated for corrective maintenance with ASAP transport strategy.
} 

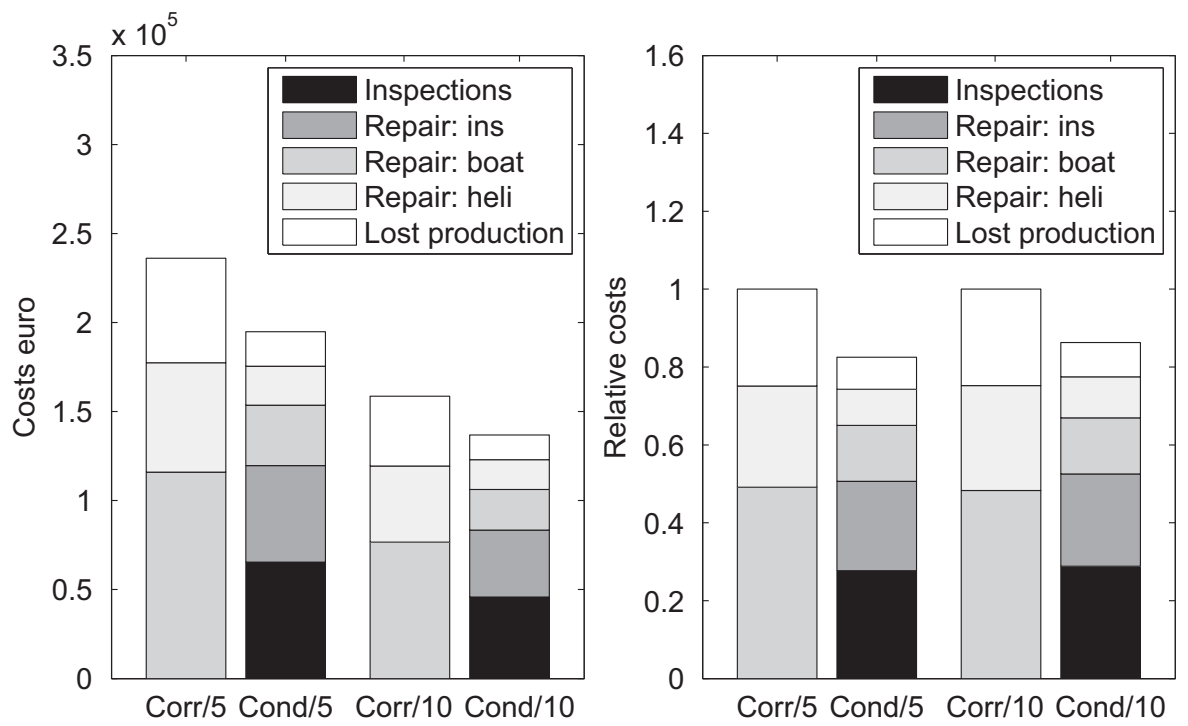

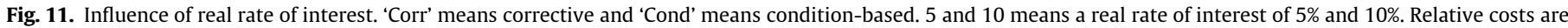
normalized with respect to the total costs for corrective maintenance.
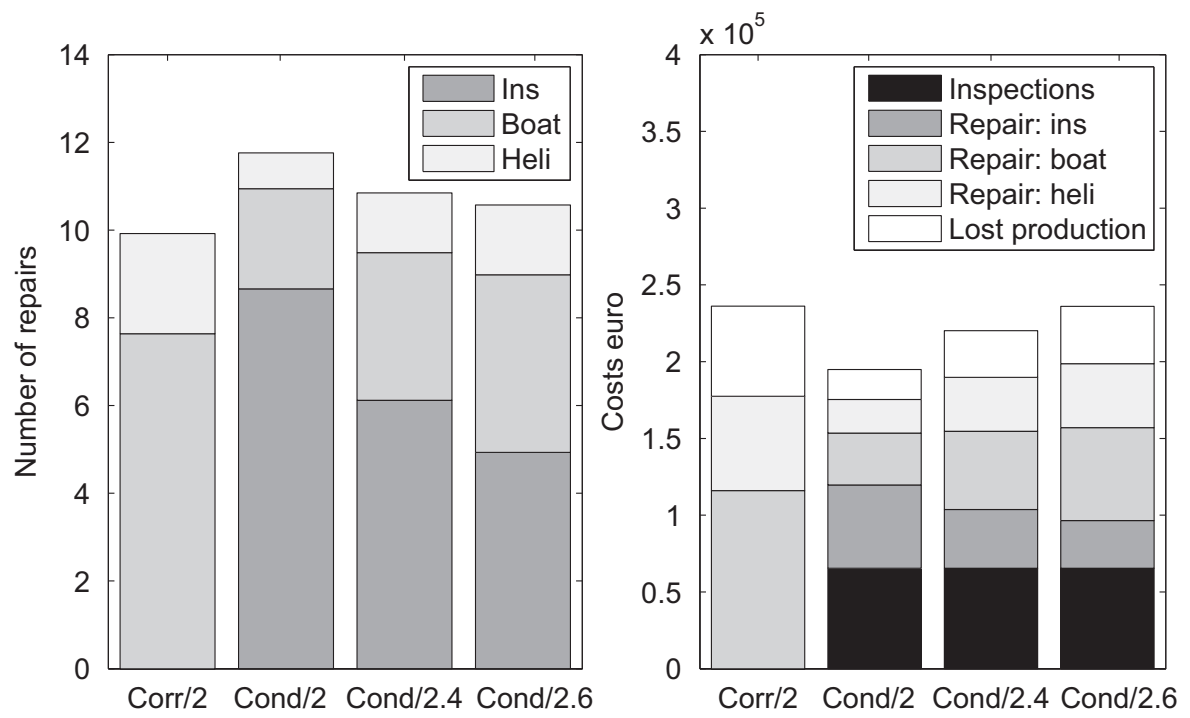

Fig. 12. Influence of damage parameter $m$. 'Corr' means corrective and 'Cond' means condition-based, 2, 2.4, and 2.6 are the $m$-values.

decrease. To investigate the relative costs of condition-based maintenance compared to corrective, analyzes have been made with a MTBF of 2 and 4 years, and are shown in Fig. 13. The costs shown to the right in Fig. 13 are normalized in a similar way as in Fig. 11. The figure shows that the costs due to inspections and condition-based repairs are so large for a MTBF of 4 years, that they almost equals the savings due to less corrective repairs. This shows that the inspection interval should be increased if the expected MTBF is larger. This has been evaluated in Fig. 14, where the optimal inspection interval for a MTBF of 4 years has been found to be 12 months.

\subsection{Influence of costs}

The specific costs due to inspections, repairs and lost production highly influence the relative costs due to conditionbased repairs compared to corrective. A measure for the influence on the total cost is the elasticity coefficient given as

$e_{p}=\frac{d C}{d p} \frac{p}{C}$

where $C$ is the total cost and $p$ is the parameter of interest, e.g. a specific cost. The elasticity coefficient gives the change of $C$ in\%, when $p$ is changed $1 \%$, and is thus a measure of the marginal cost.

The value can be computed directly from the distribution of the costs for the cases with the transport strategies boat and ASAP. For the risk-based transport strategy the decision rule is sensitive to the specific costs, and thus the distribution of the costs is dependent on the specific costs.

In Table 4 the elasticity coefficients are shown for corrective and condition-based maintenance. For corrective maintenance with boat the price of repair by boat and the cost of energy are most important, as they are the only costs. For the corrective ASAP-strategy the price of repairs by helicopter is most important. For the condition-based strategies the inspection costs and repairs 

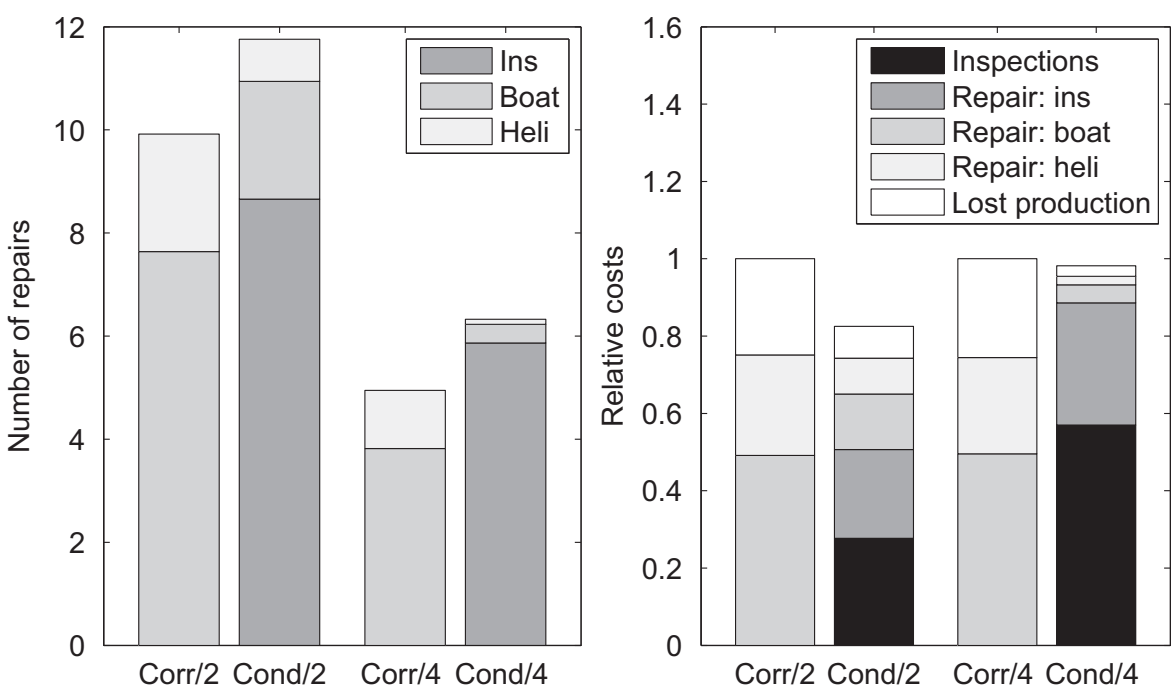

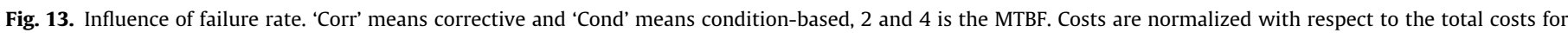
corrective maintenance.
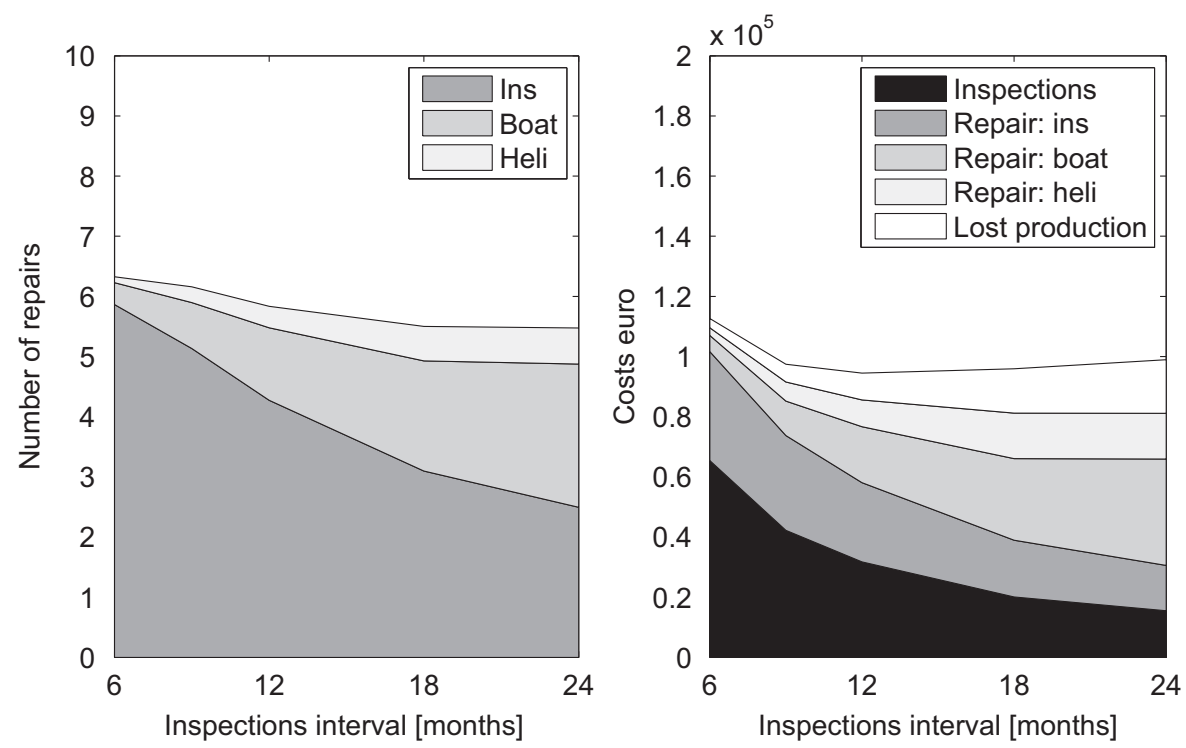

Fig. 14. Influence of inspection interval for MTBF of 4 years.

Table 4

Elasticity coefficients.

\begin{tabular}{llllll}
\hline Strategy & Ins & Repair: ins & Repair: boat & Repair: heli & Energy \\
\hline Corr, boat & 0 & 0 & 0.50 & 0 & 0.50 \\
Corr, ASAP & 0 & 0 & 0.10 & 0.78 & 0.12 \\
Cond, boat & 0.30 & 0.24 & 0.22 & 0 & 0.24 \\
Cond, ASAP & 0.32 & 0.26 & 0.04 & 0.33 & 0.06 \\
\hline
\end{tabular}

by inspections are important, and this decreases the importance of boat and energy for the strategy with boat, and for helicopter for ASAP.

\section{Discussion}

The model described in this paper concerns a generic case with only one component in one wind turbine. In other application areas system effect has been modeled, e.g inspection planning for offshore jacket structures, see [14,15], and maintenance planning for bridges, see $[16,17]$. This section describes some limitations of the presented model, and how the model can be expanded to take further aspects into account.

\subsection{Extension to more components}

In order to capture the availability and the total maintenance and operation costs of the entire wind turbine (or wind farm), it is necessary to include in the model all components that are significant contributors to the cost and the downtime. It is possible to use the described model for the individual components, where a damage model in the form of Eq. (4) can be used to describe the deterioration process. It is noted that this is not the case for many electrical components where no model for the damage accumulation can be formulated-for these components corrective maintenance or scheduled preventive maintenance can be used instead. But for many other components a damage model can be established, and the influence from wind and waves, 
respectively, can be taken into account when modeling the damage accumulation for the different components.

When considering an entire wind turbine with many components the following extensions of the model presented in this paper should be considered:

1. Cost and decision model: basically the cost should be split into base costs and marginal costs for each additional component, and in a similar way the decision parameters describing the inspection and O\&M strategy can be split into common decision parameters and decision parameters related to each component.

2. Stochastic model and reliability: some stochastic variables will be common (correlated) for all components (e.g. parameters describing the weather) whereas others will be only partly correlated (e.g. parameters describing the strength of each component). Further, besides the component reliability also the system reliability is needed.

In a model with many components the cost model should take into account that it will be cheaper to inspect more components at the same time, as the transport should only be paid once. Instead of having a constant cost for the inspection, $C_{I N S P}$ could be formulated as

$C_{I N S P}=C_{T R}+\sum_{i=1}^{n} C_{I C, i}$

where $C_{T R}$ is the cost of transport and $C_{I C, i}$ is the unit cost of inspecting the $i$ th component.

In the model it is assumed that condition-based repairs are performed the same day as an inspection. If more components are inspected the same day, they can all be repaired, but the unit cost for each repair will be the same as in the case, where they were inspected different days. Therefore the repair cost for conditionbased repairs should be found as the sum of the cost for the individual repairs. This implies that the optimal repair limit can be found for each component separately, if the inspection interval is given. This assumption requires that there is sufficient time for repairing all components with failures on the given day.

The optimal inspection interval would in general be larger for components with larger MTBF, as shown in Fig. 14. However, if more components are inspected at the same time, the cost of the transport only has to be paid once, and then it will be cheaper to inspect additional components at the same time. Consider two components where the optimal inspection intervals are 6 and 12 months, respectively, if they are considered separately. If they are considered together it is taken into account that the additional cost of inspecting the second component at 6 month intervals as well is relatively small, and that might give smaller total costs, as more corrective repairs could be avoided. Therefore the optimal inspection interval for each component should optimally be adjusted in a model with more components.

\subsection{Extension to a wind farm}

The above extension is also possible when considering a wind farm. The number of decision parameters will increase significantly and in general be quite difficult to handle by decision theoretical tools. However, for some of the major components a joint planning of operation and maintenance for the whole wind farm can still be done on the same decision theoretical basis as described above considering the extensions in items 1 and 2 in Section 8.1 .

In a model with many wind turbines in a wind farm it would be reasonable to have the same inspection interval and repair limit for equal components in all wind turbines. Condition-based repairs could still be performed with respect to the same decision rule.

A further extension could make it possible to investigate the influence of the number of available inspection/repair teams, the number/type of vessels for different repair operations, the number of spare parts in storage, etc. This would make a more detailed handling of time requirements for transport, inspections, and repairs necessary. For a wind farm the possibility of having a platform with a crew hotel could be investigated, so that long weather windows are not necessary, if a repair action can be divided into smaller parts.

\subsection{Other extensions}

A realistic situation with an uncertain weather forecast can be included, where the weather forecast is uncertain at the time of a decision. This means that the weather might go bad during a longer repair which will cause the repair costs to increase. This also makes it more difficult to make a decision on when to wait for good weather for boat and when to go by helicopter.

The inspections could be supplemented with online monitoring, where information on the health of different components is available at all times, but with larger uncertainty than the inspections. This would also make it very relevant to include a probability of false indications, and the decision rule regarding repairs will be different from the one where the damage is found at inspections. The inclusion of measurement uncertainty could also influence the performance of a strategy.

Future work could aim at developing generic O\&M plans with optimal inspection/monitoring plan, $e$, and optimal decision rules on repairs, $d(S)$, as a function of important generic parameters e.g. MTBF, and $C_{R} / B$, where $B$ is the total benefit per year, as it has been done for risk-based inspection planning of hot spots in offshore steel structures, see [18].

\section{Conclusions}

In this paper the use of condition-based maintenance has been compared to the use of corrective maintenance for generic offshore wind turbines. For illustration only one component in one wind turbine is considered. Inspections were assumed to be performed at service visits, and repairs were made if the damage was found to exceed a limit value. The condition-based strategy was found to give a larger number of repairs through the lifetime of the structure, but most corrective repairs could be avoided.

A parameter study was utilized to investigate the influence of different parameters. The value of the repair limit influences the number of condition-based and corrective repairs and the optimal value was found to be $0.1-0.2$ for the given example. A more reliable inspection will also decrease the number of corrective repairs, and the pay-off depends on the cost of making the more reliable inspection. The optimal inspection interval is dependent on the failure rate, as a small inspection interval will give large inspection costs compared to the gain from less corrective repairs. Condition-based maintenance is less efficient for large values of the damage exponent, as the damage suddenly increases too fast to be detected at the inspections. The real rate does not influence the relative costs between condition-based and corrective maintenance significantly. The used specific costs are fictive, and a change will influence the costs for the different strategies. 


\section{Acknowledgements}

The work presented in this paper is part of the project "Reliability-based analysis applied for reduction of cost of energy for offshore wind turbines" supported by the The Danish Council for Strategic Research, Grant no. 2104-08-0014. The financial support is greatly appreciated.

\section{References}

[1] Nilsson J, Bertling L. Maintenance management of wind power systems using condition monitoring systems. IEEE Transactions on Energy Conversion $2007 ; 22(1)$.

[2] Rademakers L, Braam H, Obdan T. Estimating costs of operation \& maintenance for offshore wind farms. This paper had been presented at the EWEC 2008, 2008.

[3] Walford C. Wind turbine reliability: understanding and minimizing wind turbine operation and maintenance costs. Technical Report, SANDIA Report SAND2006-1100; 2006.

[4] Giebhardt J. Predictive condition monitoring for offshore wind energy converters with respect to the IEC61400-25 standard. This paper had been presented at the DEWEK 2004, 2004.

[5] Sørensen JD. Framework for risk-based planning of operation and maintenance for offshore wind turbines. Wind Energy 2009;12(5):493-506.

[6] Raiffa H, Schlaifer R. Applied statistical decision theory. Cambridge: Harvard University Press, Cambridge University Press; 1961.

[7] Wiggelinkhuizen E, Verbruggen T, Braam H, Rademakers L, Xiang J, Watson S Assessment of condition monitoring techniques for offshore wind farms.
Journal of Solar Energy Engineering, Transactions of the ASME 2008;130(3):0310041-9.

[8] Risø DTU. WAsP - the Wind Atlas Analysis and Application Program < http:// www.wasp.dk>, 2008.

[9] Straub D. Generic approaches to risk based inspection planning for steel structures. PhD thesis, Swiss Federal Institute of Technology, ETH Zurich; 2004.

[10] Pinson P. Estimation of the uncertainty in wind power forecasting. PhD thesis, Mines ParisTech; 2006.

[11] Costa A, Crespo A, Navarro J, Lizcano G, Madsen H, Feitosa E. A review on the young history of the wind power short-term prediction. Renewable and Sustainable Energy Reviews 2008;12(6):1725-44.

[12] Rademakers L, Braam H, Obdan T, Pieterman RV. Operation and maintenance cost estimator (OMCE)—final report. Technical Report, ECN Report ECN-E-09037; 2008

[13] Faulstich S, Hahn B, Jung H, Rafik K, Ringhandt A. Appropriate failure statistics and reliability characteristics. This paper had been presented at the DEWEK 2008, 2008.

[14] Goyet J, Straub D, Faber M. Risk-based inspection planning of offshore installations. Structural Engineering International 2002:3:200-8.

[15] Straub D, Faber M. System effects in generic risk-based inspection planning. Journal of Offshore Mechanics and Arctic Engineering 2004;126:265-71.

[16] Frangopol D, Liu M. Maintenance and management of civil infrastructure based on condition, safety, optimization, and life-cycle cost. Structure and Infrastructure Engineering 2007;3(1):29-41.

[17] Neves L, Frangopol D, Cruz P. Multi-objective probabilistic optimization of bridge lifetime maintenance: novel approach. In: Proceedings of the 3rd international conference on bridge maintenance, safety and managementbridge maintenance, safety, management, life-cycle performance and cost; 2006. p. $539-41$.

[18] Straub D, Faber M. Computational aspects of risk-based inspection planning. Computer-Aided Civil and Infrastructure Engineering 2006;21:179-92. 


\section{A.2 Paper 2}

Title:

Planning of O\&M for Offshore Wind Turbines using Bayesian Graphical Models

Authors:

Nielsen, J. J. and J. D. Sørensen.

Conference:

ESREL 2010 annual conference, Rhodes, Greece. 



\title{
Planning of O\&M for offshore wind turbines using Bayesian graphical models
}

\author{
Jannie Jessen Nielsen \& John Dalsgaard Sørensen \\ Department of Civil Engineering, Aalborg University, Denmark
}

\begin{abstract}
The costs to operation and maintenance (O\&M) for offshore wind turbines are large, and riskbased planning of O\&M has the potential of reducing these costs. This paper presents how Bayesian graphical models can be used to establish a probabilistic damage model and include data from imperfect inspections and monitoring. The method offers efficient updating of the failure probability, which is necessary for risk-based decision making. An application example is presented to demonstrate the capabilities of the method.
\end{abstract}

\section{INTRODUCTION}

The costs to operation and maintenance (O\&M) of offshore wind turbines are large contributors to the cost of energy. These costs can be reduced if better maintenance strategies are used. Presently component failures cause large costs to corrective maintenance. Failure of even a minor component might cause escalated damage, and the wind turbine stops producing power until a repair or replacement is utilized. Because repairs of offshore wind turbines require good weather conditions, the harsh environment often delays the repair, and the production of energy is reduced. The costs to lost production and repairs can be reduced if preventive maintenance strategies are used, so damages are repaired before failure occurs.

Condition based maintenance is a preventive maintenance strategy where decisions on repairs are made based on the actual condition of the components. This knowledge is obtained using condition monitoring. There exist a large number of condition monitoring methods that in general can be divided into offline inspections and online monitoring, see (Walford 2006). A review of condition monitoring methods for wind turbines is given in (Hameed et al. 2009). The benefit of condition monitoring has been quantified in (McMillan and Ault 2007), and the benefit was found to be highly dependent on the reliability of the condition monitoring, which was not modeled explicitly. In the project CONMOW it was concluded that the monitoring methods still need development in order to detect failures reliably (Wiggelinkhuizen et al. 2008).

Optimal planning of O\&M should be based on riskbased methods, where all information from past experience and condition monitoring is taken into ac- count, and the uncertainties are modeled as realistic as possible, see the framework for risk-based O\&M for wind turbines in (Sørensen 2009). Rational decisions can be made based on a pre-posterior decision analysis where the expected costs through the lifetime of the wind turbines are minimized, see theory in (Raiffa and Schlaifer 1961).

For components exposed to deterioration processes damage models can be used to describe the development in damage size with uncertain parameters described by stochastic models. Damage models are in general associated with large uncertainties, and information from (imperfect) inspections/online monitoring should be included to make a more reliable posterior estimate on the damage size. The Bayesian updating can efficiently be done using Bayesian graphical models, and has earlier been used for deterioration modeling, see (Friis-Hansen 2000) and the framework in (Straub 2009). This paper presents how Bayesian graphical models can be used to assist in decision problems for O\&M of offshore wind turbines, and discusses how to include data from inspections and monitoring properly.

\section{DECISION MODEL}

Decisions on inspections and repairs should be made such that the expected costs through the lifetime are minimized. For different inspection plans/methods and different decision rules for repairs the expected costs can be calculated based on a decision tree. For wind turbine components it is relevant to consider both corrective repairs that are required if failure occurs, and preventive repairs, which can be made based on the inspection/monitoring results. If it is assumed 
that a repaired component behaves like a new component, the simplified decision tree in figure 1 can be used.
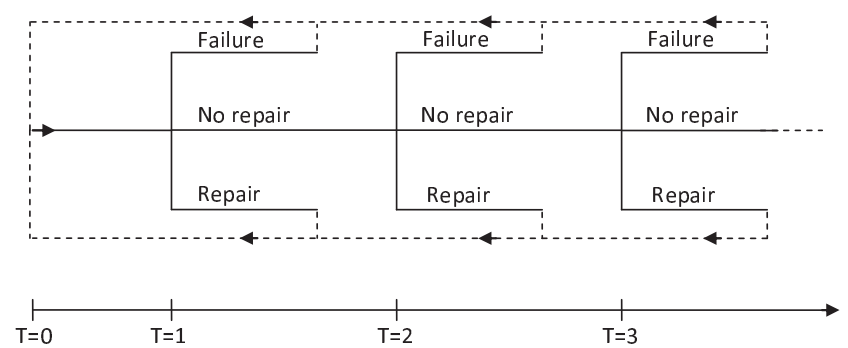

Figure 1: Simplified decision tree.

The decision tree should be expanded to correspond to the design life of the wind turbine. If either repair type is performed, continuation should be made from the beginning of the tree, but now the length of the tree should be the remaining life time. Based on the decision tree with the associated utilities and probabilities, the optimal inspection plan and decision rule can be found among a finite set of alternatives, see e.g. (Straub 2004).

\section{DETERIORATION MODELING}

The primary focus should be on components that are large risk contributors. In general offshore wind turbine operators do not make failure data public available, and third parties have to be content with the limited available material. For onshore wind turbines public databases with failure data are available, e.g. from the scientific measurement and evaluation programme (WMEP), see (Faulstich et al. 2009). For a generic offshore project the distribution of risk from different contributors, divided by component and failure type, was shown in (Skjærbæk et al. 2009), and data from different sources were collected in (McMillan and Ault 2007).

In general most failures are caused by electric components, control system, sensors and hydraulic system. These failures are critical for offshore wind turbines because of the limited accessability. Failures from large components, i.e. gearbox, generator, main shaft/bearing, and rotor, are rare, but the risk is high because they cause long downtime, require large vessels, and the spare parts are expensive.

\subsection{Damage models}

In order to use risk-based planning of O\&M it is necessary to establish a probabilistic damage model, which allows for the calculation of the failure probability. Appropriate damage models requires knowledge of the relevant failure types, what the cause (load) is, and how they progress. The data for the prior damage model can be obtained from theoretical mod- els, experiments, and measured data for similar components.

Components exposed to fatigue are typically designed using SN-curves combined with the PalmgrenMinor damage accumulation law. This model cannot be used directly for risk-based inspection planning, because it does not include a measurable damage size. Therefore a fracture mechanical (FM) approach is necessary, e.g. Paris law for crack propagation:

$\frac{d a}{d N}=C \cdot \Delta K^{m}$

This model gives the increase in damage per stress cycle, $d a / d N$, as function of the stress intensity factor range, $\Delta K$, and parameters $C$ and $m$.

The FM curve can be calibrated to the SN-model so the probability of failure or reliability index is equivalent for the two models, see e.g. (Straub 2004). This approach have been used in the context of risk-based inspection planning of offshore steel jacket structures. For wind turbine components exposed to other deterioration processes a similar calibration to a crack propagation law can be utilized.

The failure limit state could for a specific component be chosen to the damage size that causes the wind turbine to stop production. Another important limit state is the damage level where it is not possible to repair a damage, and instead requires a component exchange because of escalated damage.

\subsection{Condition monitoring}

Condition monitoring is essential for updating of the damage model in accordance with the actual state of the component. Modern wind turbines are equipped with a supervisory control and data acquisition (SCADA) system that monitors production, start and stops, occurrences of alarms, and measurements of e.g. temperature, tower vibrations, and fluid levels. More advanced equipment are increasingly used, i.e. vibration monitoring of bearings and gearbox, and monitoring of fluid contamination, and other possible techniques are strain measurement using optic fibers, crack detection using acoustic emission sensors, see (Walford 2006) and (Hameed et al. 2009). In addition a number of offline monitoring methods are available, e.g. analysis of oil samples, thermography of electric components, and visual inspections.

In general the measurements obtained using condition monitoring are indicators of the health of different components, or indicators of the load. Particles in the gearbox oil indicates the extend of wear, vibration measurements can indicate damages in the drive train, and power production and tower vibration measurements gives indications of the loads from wind and waves. All this data can in principle be used to update the damage model, but as the measurements 
are not direct nor perfect measurement of the damage size, the uncertainties should be taken into account in the model.

For methods that gives an indication on whether a damage is present or not, the probability of detecting a damage can be modeled by a PoD-curve that gives the probability of detection as function of the damage size. An example is an exponential model with parameters $P_{0}$ and $\lambda$, see e.g. (Straub 2004):

$$
\operatorname{PoD}(a)=P_{0}(1-\exp (-a / \lambda))
$$

For methods where a damage size or extend of damage can be measured, the model should take the measurement accuracy into account. This can be done by either a additive or a multiplicative model. A simple additive model assumes that the correct damage size, $a$, equals the measured size, $a_{m}$, plus an error term given as a normal distributed random variable with mean zero, $\varepsilon$ :

$a=a_{m}+\varepsilon$

The indirect load measurements can be included in a similar way with a measurement error on e.g. the stress intensity factor range, $\Delta K$, in equation 1 .

\section{BAYESIAN NETWORKS}

A Bayesian Network is a graphical modeling tool that originated in computer science for modeling of artificial intelligence, introductions can be found in e.g. (Murphy 2001) and (Jensen and Nielsen 2007). The name Bayesian refers to the well known Bayes rule for calculation of a the probability of event $A$, given event $B$ :

$$
P(A \mid B)=\frac{1}{P(B)} P(B \mid A) P(A)
$$

In the context of Bayesian updating, $P(A \mid B)$ is the posterior distribution of $A$ given $B, P(A)$ is the prior distribution of $A$, and $P(B \mid A)$ is the likelihood of $A$ given $B$.

A Bayesian network consist of a set of variables, graphicly shown as nodes, and their causal relationships, shown using directed links. Together the nodes and links form a directed acyclic graph (DAG). If a variable $A$ causes $B, A$ is a parent of $B$, which is a child of $A$. Figure 2 shows a simple Bayesian network with three nodes, where $A$ is a parent of $B$ and $C$. If evidence is received about node $A$, it will change the belief about node $B$ and $C$. If instead information about $B$ is found, it will give indirect information about $A$, therefore also change the belief about $C$.

The probability distributions are given as a conditional distribution for each variable given the parents. The joint distribution for a network with $n$ nodes,

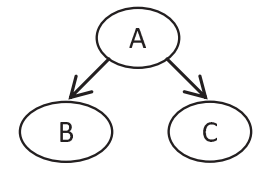

Figure 2: Simple Bayesian network.

$P(V)$, can be found using the chain rule for Bayesian networks:

$P(V)=\prod_{i=1}^{n} P\left(A_{i} \mid p a\left(A_{i}\right)\right)$

where $A_{i}$ is the $i$ 'th variable in the network, and $p a\left(A_{i}\right)$ is the parents of $A_{i}$. When knowledge about the value/state of a variable becomes available, it can be entered in the network, and the marginal posterior distribution of each node can be found using Bayes rule. The calculation on posterior probabilities in Bayesian networks is called inference.

In order to model deterioration the network should allow the damage size to increase with time. This can be done using a dynamic Bayesian network, that consist of equal time slices, one for each time step. Each time slice is connected only to the neighboring slices, and will thus be independent of all other past slices given the previous slice. This equals the property of a Markovian process, but is general deterioration processes are not Markovian. However (Straub 2009) used a dynamic Bayesian network with time independent parameters equal for each slice to make the Markovian assumption hold.

\subsection{Inference}

The variables used for modeling of deterioration are generally continuous. In general exact inference is not possible for a network with continuous nodes, as opposed to networks with discrete nodes, where efficient algorithms exist, see (Murphy 2002). Therefore the variables have been discretized in previous applications where deterioration processes have been modeled using Bayesian networks, see (Friis-Hansen 2000) and (Straub 2009). But even though the inference can be performed exact, the discretization introduces an approximation for the probability distributions. The nodes without parents, e.g. damage variables, can be truncated without approximation. But in general discretization of conditional distributions introduces an error. The damage size at one time step is in the model in section 3.1 assumed to be a deterministic function of the parents. For a discrete model a conditional probability distribution has to be found, which performs an operation corresponding to the deterministic function. For each combination of states of the node representing the damage size at time $t-1$ and the nodes representing parameters in the damage model, the probability that the damage size at time step $t$ is in 
each state should be calculated, and this can be done using sampling. The accuracy of this conditional distribution highly affects the final result when inference is performed.

An alternative to the discrete models is to use continuous model, where approximate inference methods are needed. Markov Chain Monte Carlo (MCMC) methods can be used to handle continuous distributions, e.g. the Gibbs sampling algorithm, see (Gelfand and Smith 1990). The method was earlier considered not to be usable for deterioration modeling partly because it does not allow deterministic/logic relations among variables according to (Hrycej 1990). This problem has been overcome in the program WinBugs (Lunn et al. 2000), where it is possible to include logic nodes, that are deterministic functions of other nodes. These nodes are not considered as being variables, and are not allowed to receive evidence. In the Gibs sampling iteration scheme values are not drawn from the logic nodes, but the logic relationship are used to calculate the variables needed for the calculations.

The evaluation is performed via simulation from a Markov chain that has the property that the stationary distribution is the posterior. In Gibbs sampling, sampling is performed from the full conditional distributions. In a Bayesian network a node is independent of all other nodes given the Markov blanket (parents, children, and parents of children). Thus the full conditionals only contain these nodes, and can be evaluated as (Lunn et al. 2000):

$$
P(v \mid V \backslash v) \propto P(v \mid p a(v)) \times \prod_{w \in \operatorname{ch}(v)} P(w \mid p a(w))
$$

where $\operatorname{ch}($.$) means the children of. Since all distri-$ butions in a Bayesian network is given conditional on the parents, the distributions used for calculating the full conditionals are known. Gibbs sampling is performed by first choosing an initial value for all variables. In a loop over all variables a new value is sampled for each variable, given the value of all other variables, so that the variables are updated in turn. For three variables $X, Y, Z$ the updating can be performed in the following scheme, where $\sim$ means "drawn from":

$$
\begin{aligned}
X_{i} & \sim P\left(X \mid Y_{i-1}, Z_{i-1}\right) \\
Y_{i} & \sim P\left(Y \mid X_{i}, Z_{i-1}\right) \\
Z_{i} & \sim P\left(Z \mid X_{i}, Y_{i}\right)
\end{aligned}
$$

In the first rounds of the loop the samples are influenced by the initial values, $X_{0}, Y_{0}, Z_{0}$, but after a burn in period the set of values sampled using Gibbs sampling can be shown to be a sample from the true posterior distribution (Gelfand and Smith 1990).

\section{APPLICATION EXAMPLE}

This example demonstrates how Bayesian networks can be used to update damage size and failure probability, when new information becomes available, and be used for risk-based repair planning. A specific case relevant for wind turbine O\&M is considered, but the model is in principle generic, and can easily be changed to model other cases.

The considered failure mechanism is fatigue crack growth in a main bearing with a mean time between failures (MTBF) of 10 years. The crack growth is considered to be governed by the wind load on the wind turbine, which is monitored in the SCADA system. Further visual offline inspections are performed once a year. Failure is defined as the crack size, that makes the system shut down, and stops power production. Thus it is detected if failure occurs. Failure is assumed to occur at a crack size of $20 \mathrm{~mm}$.

\subsection{Damage model}

The damage model considered is based on Paris' law, equation 1. In this example the formulation used in (Friis-Hansen 2000) is used. Here the stress ranges are assumed to follow a Weibull distribution and the differential equation is solved to yield the following expression for the crack size at the $i$ 'th time step, $a_{i}$, given the crack size at previous time step, $a_{i-1}$ :

$a_{i}=\left(a_{i-1}^{\frac{2-m}{2}}+\Delta K M_{U} A_{t}^{m}\right)^{\frac{2}{2-m}}$

where the stress intensity factor range, $\Delta K$, is found using:

$\Delta K=C N \Gamma\left(1+\frac{m}{B}\right) Y^{m} \pi^{\frac{m}{2}}\left(1-\frac{m}{2}\right)$

$C$ and $m$ are damage parameters, $A$ and $B$ is scale and shape parameter for the Weibull distribution, $Y$ is a geometry constant, $N$ is the number of load cycles in each time step, and $M_{U}$ models the time independent model uncertainty. The effect of the control system of the wind turbine is assumed to be included in the load model.

The values and distributions for the parameters are given in table 1 . Most of the variables are taken as given in (Friis-Hansen 2000), but $C$ is calibrated using Crude Monte Carlo simulations with a time step of 3 months to give the assumed MTBF of 10 years.

\subsection{Bayesian network}

The Bayesian network is modeled in the program WinBugs. The model consist of stochastic nodes, logic nodes and constants, but only the stochastic and logic nodes are shown in the graphical model. In addition to the continuous variables necessary for the damage model, a discrete logic failure node is made 


\begin{tabular}{llll}
\hline Variable & Distribution & Mean & CoV \\
\hline$m$ & Deterministic & 3 & - \\
$C$ & Deterministic & $6 \cdot 10^{-12}$ & - \\
$B$ & Deterministic & 0.66 & - \\
$Y$ & Deterministic & 1 & - \\
$N$ & Deterministic & $2.5 \cdot 10^{5} /$ year & - \\
$a_{0}$ & Exponential & $1 \mathrm{~mm}$ & $100 \%$ \\
$A_{i}$ & Normal & $5.35 \mathrm{MPa}$ & $18 \%$ \\
$M_{U}$ & Normal & 1 & $18 \%$ \\
\hline
\end{tabular}

Table 1: Distributions for damage parameters.

for each time step with the two states, 0 and 1 , corresponding to no failure and failure respectively. The mean of the failure node is thus the failure probability.

The indicators available in this example are:

- An inspection every year: uncertain measurement of $a_{i}$ (additive measurement error)

- Continuous load measurement: uncertain measurement of $A_{i}$ (additive measurement error)

- If no failure has occurred, it is known

In order to take a measurement accuracy into account, a stochastic node has to be included for $a_{i}$ and $A_{i}$ with the names $a_{m, i}$ and $A_{m, i}$ respectively. They are both considered to be normal distributed with the true value as mean, and standard deviations $\sigma_{a}=0.5$ $\mathrm{mm}$ and $\sigma_{A}=0.25 \mathrm{MPa}$. This correspond to an additive measurement error, and is a more convenient notation in WinBugs.

The failure node, $F_{i}$, cannot directly receive the evidence that failure has not occurred, because a logic node cannot receive evidence. Therefore a stochastic node also has to be made for the observation of the failure node, and it is called $F_{m, i}$. This node follows a Bernoulli distribution, where the probability of an observation that is failure, is equal to the state of the failure node. If the state of the failure node is 1 , the probability that $F_{m, i}$ is 1 , is 1 . If the state of the failure node is 0 , the probability that $F_{m, i}$ is 1 , is 0 .

The nodes $M_{U}, a_{0}$, and $A_{i}$ have no parents, and their prior distributions are already given in table 1. The distribution types of the other nodes are summarized in table 2, and the Bayesian network is shown in figure 3.

The time step is chosen to be 3 months. The Bayesian network is made for 10 years, and thus consist of 40 time slices. This gives a relatively low computation time and the time step is short enough to make use of information from monitoring between the annual inspections.

A burn in period of 5000 samples and additional 25000 samples for the calculation of posterior distributions, have been found appropriate to give a sufficient good approximation of the failure probability.

\begin{tabular}{lll}
\hline Variable & Distribution & Parameters \\
\hline$a_{i}$ & Logic & Eqn. 8 \\
$F_{i}$ & Logic & 1 if $a_{i}>20 \mathrm{~mm}$ \\
$a_{m, i}$ & Normal & $\mu=a_{i}, \sigma=0.5 \mathrm{~mm}$ \\
$A_{m, i}$ & Normal & $\mu=A_{i}, \sigma=0.25 \mathrm{MPa}$ \\
$F_{m, i}$ & Bernoulli & $p=F_{i}$ \\
\hline
\end{tabular}

Table 2: Distributions for nodes with parents.

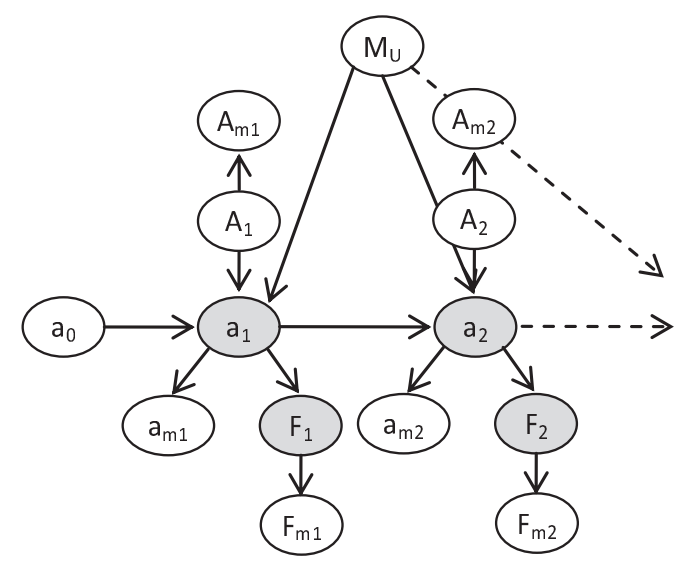

Figure 3: First two time slices of the Bayesian network. Grey nodes are logic nodes, white nodes are stochastic.

\subsection{Decision rule}

In principle the decisions on repairs should be made based on a life cycle analysis with a decision tree as explained in section 2, where it is possible to take the limited life time into account. For simplicity a more simple decision rule is chosen for this example. This decision rule is set as a limit value of the probability of failure until next inspection. The limit value is set such that the expected total costs for repairs and failure per year is equal for the case where a repair is performed at time $t$, and the case where the repair is postponed a year to time $t+\Delta t$. The expected annual costs for a repair performed at time $t$ is:

$E\left[C_{t}\right]=\frac{C_{R}}{t}$

where $C_{R}$ is the cost of a preventive repair. If the repair instead is postponed to time $t+\Delta t$, there is a probability of failure, $P_{F}$, and the annual expected costs are:

$E\left[C_{t+\Delta t}\right]=\frac{C_{F} \cdot P_{F}+C_{R}\left(1-P_{F}\right)}{t+\Delta t}$

where $C_{F}$ is the cost of failure. As the cost of failure is larger than the cost of a preventive repair, the numerator of the second expression is always larger than for the first. But it is divided with a longer period, because the repair is postponed one year. For each value of $t$ the 'optimal' limit failure probability, $P_{F L}$, can 
be found by setting $E\left[C_{t}\right]=E\left[C_{t+\Delta t}\right]$, which yields:

$$
P_{F L}(t)=\frac{\Delta t}{t\left(\frac{C_{F}}{C_{R}}-1\right)}
$$

In the example the ratio $C_{F} / C_{R}$ is set to 5 .

\subsection{Results}

Application of the above decision rule requires the evaluation of the failure probability. In a situation where no inspections and no load monitoring is available, the cumulative distribution of the failure time is shown in figure 4 , updated in year 1 to 4 with the information that no failure has yet occurred. The thick black line shows the cumulative distribution reset for each year, and can be compared with the limit value for repairs, which is shown with the dashed line. As the two lines intersect before four years, the decision after observation in year three should be to repair.

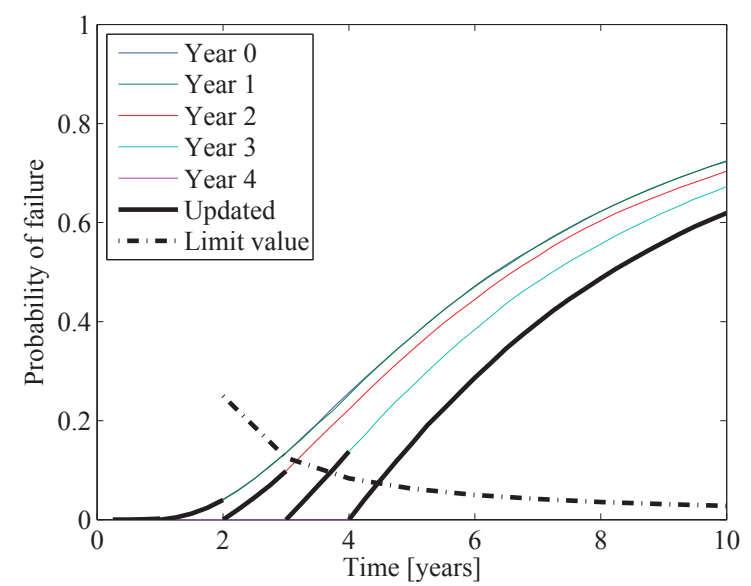

Figure 4: Cumulative distribution function of failure time for case without inspections.

In order to illustrate a situation where inspections are available for updating of the damage model, a realization from the population has been chosen as case study. The damage size and the uncertain observation for each year until failure occurs are shown in table 3 for the specific realization. These observations and the observation that no failure has occurred yet are entered for one year at a time as evidence in the model. The resulting failure probabilities are shown in figure 5 , where the model is updated with new evidence in year one to four. The thick black line again shows the cumulative distribution reset for each year. In the case without observations the probability of failure in the third, fourth, and fifth year were each year in the range $0.10-0.15$. In the case where observations are used to update the model, the annual probability of failure is very low until the fifth year, and the updated probability of failure in the fifth year is more than 0.9. Even

\begin{tabular}{llllll}
\hline Year & 1 & 2 & 3 & 4 & 5 \\
\hline$a[\mathrm{~mm}]$ & 1.10 & 1.75 & 3.56 & 10.10 & fail. \\
$a_{m}[\mathrm{~mm}]$ & 0.7 & 1.8 & 3.6 & 9.3 & fail. \\
\hline
\end{tabular}

Table 3: Realizations of the damage size $(a)$ and the observations from annual inspections $\left(a_{m}\right)$.

without the decision rule it would be clear that the decision in year four should be to repair rather than waiting until year five.

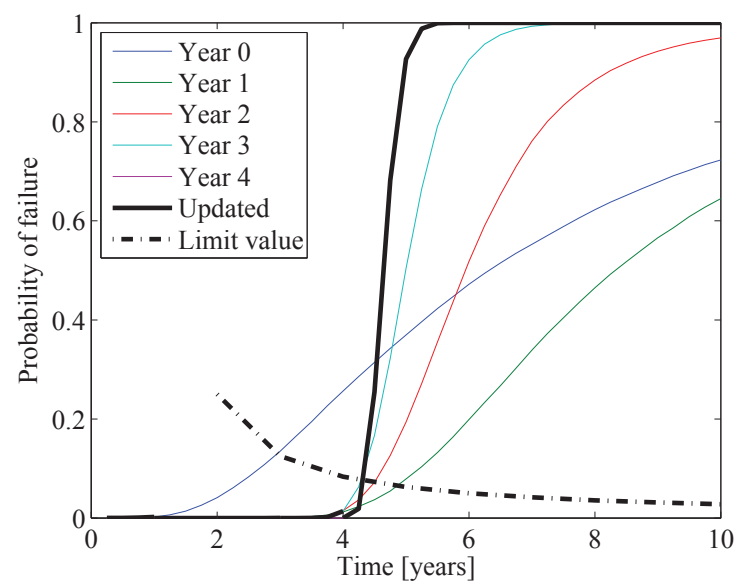

Figure 5: Cumulative distribution function of failure time in case with inspections.

Next the realizations of the uncertain measurements are inserted as evidence in addition to the inspections. Figure 6 shows a box plot of the posterior distribution of the damage size in each time step. For each time step the present and all previous observations are used for the estimate. The actual damage size for the realizations and the uncertain observations of it are also shown.

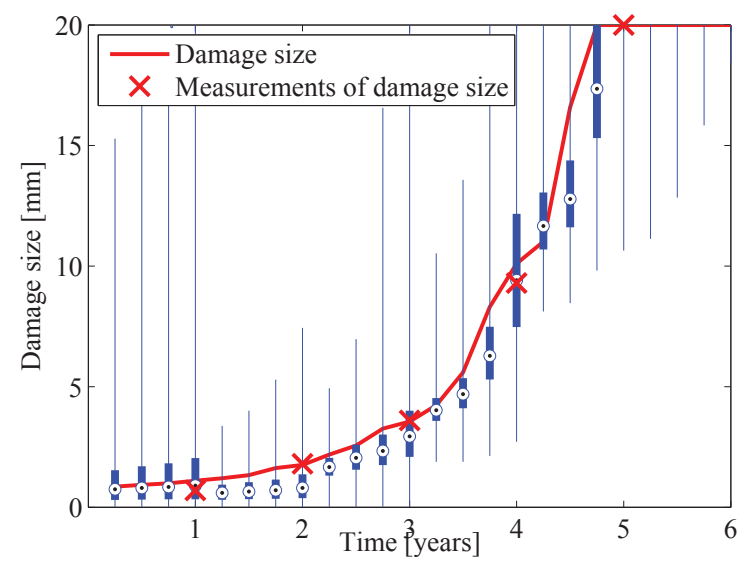

Figure 6: Box plot of damage size with inspections and monitoring. The bullets shows the medians, the filled bar is the range between the $25-75 \%$ quantiles. 
The observations at year one and four are both significantly lower than the correct damage size, caused by the randomness of the uncertain inspections. Thus the Bayesian network also expects the damage to be smaller than it actually is after year four, but the probability of it being larger is acknowledged. If the observations were trusted $100 \%$, failure would be predicted to occur later than it actually does. With a Bayesian network the uncertainties are taken into account, and the probability of failure can be used for risk-based decision making.

In figure 7 the probability of failure is shown after year four, updated for each three months. It is shown both with and without inclusion of monitoring results. It shows that the inclusion of monitoring results gives better confidence in when failure will happen, and shows that it in this case will be possible to postpone the repair half a year with only low additional risk. According to the decision rule the repair should be performed after three month, but the failure probability is still close to the limit value after half a year.

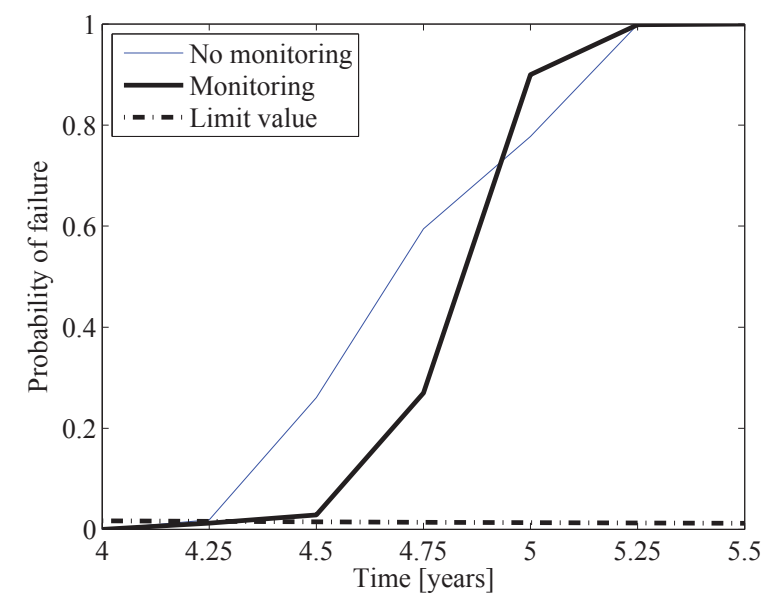

Figure 7: Probability of failure between previous and current monitoring result, based on previous monitoring result. Or in the case without monitoring only updated with the information that failure has not yet occurred.

\section{CONCLUSIONS}

This paper presents how Bayesian graphical models can be used to assist in decision problems for riskbased planning of O\&M for offshore wind turbines. It is possible to use continuous nodes in the network, when Gibs sampling is used to perform approximate inference. In this paper it has been shown that it is possible to model deterioration processes with such a network, when e.g. WinBugs is used, where it is possible to make logic nodes as well as stochastic nodes.

An application example was used to show how a Bayesian network can be used for inclusion of in- spection results and load monitoring for updating of the damage model. This gave a better estimate on the probability of failure, and could be compared to a decision rule given as a limit value of the failure probability. The model is generic and can easily be changed to include other damage and inspection models, e.g. condition monitoring results could be included. The model can also be extended to include more components, this can e.g. be relevant if one indicator is common for more components.

\section{REFERENCES}

Faulstich, S., P. Hahn, P. Lyding, and P. Tavner (2009). Reliability of offshore turbines - identifying risks by onshore experience. In European Offshore Wind 2009 Conference proceedings.

Friis-Hansen, A. (2000). Bayesian networks as a decision support tool in marine application. $\mathrm{Ph}$. D. thesis, Technical University of Denmark.

Gelfand, A. E. and A. F. M. Smith (1990). Sampling-based approaches to calculating marginal densities. Journal of the American Statistical Association 85(410), 398-409.

Hameed, Z., Y. S. Hong, Y. M. Cho, S. H. Ahn, and C. K. Song (2009). Condition monitoring and fault detection of wind turbines and related algorithms: A review. Renewable and Sustainable Energy Reviews 13(1), 1-39.

Hrycej, T. (1990). Gibbs sampling in bayesian networks. Artificial Intelligence 46(3), 351-363.

Jensen, F. V. and T. D. Nielsen (2007). Bayesian Networks and Decision Graphs. Information Science and Statistics, Springer.

Lunn, D. J., A. Thomas, N. Best, and D. Spiegelhalter (2000). Winbugs - a bayesian modelling framework: Concepts, structure, and extensibility. Statistics and Computing 10(4), 325-337.

McMillan, D. and G. W. Ault (2007). Quantification of condition monitoring benefit for wind turbine generators. Wind Engineering 31(4), 267-285.

Murphy, K. (2001). An introduction to graphical models.

Murphy, K. (2002). Dynamic Bayesian networks: Representation, inference and learning. Ph. D. thesis, University of California, Berkeley.

Raiffa, H. and R. Schlaifer (1961). Applied statistical decision theory. Harvard University.

Skjærbæk, P., T. Mousten, and H. Stiesdal (2009). Reliability assessment and improvement through arm modeling. In European Offshore Wind 2009 Conference proceedings.

Sørensen, J. D. (2009). Framework for risk-based planning of operation and maintenance for offshore wind turbines. Wind Energy 12(5), 493-506.

Straub, D. (2004). Generic approaches to risk based inspection planning for steel structures. Ph. D. thesis, Swiss Federal Institute of Technology, ETH Zurich. 
Straub, D. (2009). Stochastic modeling of deterioration processes through dynamic bayesian networks. Journal of Engineering Mechanics 135(10), 1089-1099.

Walford, C. A. (2006). Wind turbine reliability: Understanding and minimizing wind turbine operation and maintenance costs. Technical Report SAND2006-1100.

Wiggelinkhuizen, E., T. Verbruggen, H. Braam, L. Rademakers, J. Xiang, and S. Watson (2008). Assessment of condition monitoring techniques for offshore wind farms. Journal of Solar Energy Engineering, Transactions of the ASME 130(3), 0310041-0310049. 


\title{
A.3 Paper 3
}

Title:

Risk-Based Operation and Maintenance of Offshore Wind Turbines using Bayesian Networks

\author{
Authors: \\ Nielsen, J. J. and J. D. Sørensen. \\ Conference: \\ 11th International Conference on Applications of Statistics and Probability \\ in Civil Engineering, ICASP11, ETH, Zurich, Switzerland.
}





\title{
Risk-based operation and maintenance of offshore wind turbines using Bayesian networks
}

\author{
J. J. Nielsen \& J. D. Sørensen \\ Department of Civil Engineering \\ Aalborg University, Aalborg, Denmark
}

\begin{abstract}
For offshore wind farms, the costs due to operation and maintenance are large, and more optimal planning has the potential of reducing these costs. This paper presents how Bayesian networks can be used for risk-based inspection planning, where the inspection plans are updated each year through the lifetime. Two different approaches are used; one uses a threshold value of the failure probability, and one uses a Limited Memory Influence Diagram. Both methods are tested for an application example using Monte Carlo sampling, and they are both found to be efficient and equally good.
\end{abstract}

\section{INTRODUCTION}

Data from offshore wind farms shows that the costs due to operation and maintenance (O\&M) are significant contributors to the cost of energy. These costs need to be reduced in order to make offshore wind energy more competitive compared to other sources of energy. One reason for the large costs is the large number of unexpected component failures. This gives a need for corrective maintenance with direct costs of spare parts, vessels and crew, but also indirect costs due to reduced production arise. Better planning of O\&M can therefore help making wind energy more competitive.

Rademakers et al. (2009) have developed a tool for estimating the future costs due to O\&M, and to investigate, for example, the influence of having more vessels. This can be used for planning on a longer time horizon, but cannot be used for real-time planning of preventive maintenance. For real-time planning, condition monitoring can be used to gain information about the state of the components. Obdam et al. (2009) have developed a flight leader concept, where only a few wind turbines are equipped with load sensors, and the load on other turbines are estimated by comparing the SCADA data, which is collected for all wind turbines. This can be used in connection with real-time planning, but efficient methods have not yet been developed on how to use this information for preventive maintenance planning.

Rational planning should be made such that the expected costs through the lifetime are minimized, or equivalently the cost of energy (COE) is minimized. The theoretical framework for risk-based decision making has been presented by Raiffa and Schlaifer (1961), and is based on Bayesian pre-posterior analysis. This method has been used for risk-based inspection planning for offshore steel jacket structures (Faber et al. 2005), and a generic approach have been utilized to minimize the computations (Straub and Faber 2006). The inspection plans were here fixed from the beginning, and was not changed when new information from inspections was received.

Recently, Bayesian networks have been found to be efficient for deterioration modeling (Straub 2009), and can be used for risk-based inspection planning (Friis-Hansen 2000), and planning of repairs (Nielsen and Sørensen 2010). They provide efficient updating of the damage model, whenever new information from inspections and monitoring becomes available. If the network is used for planning of inspections, real-time decision making can be made such that all available knowledge is used when decisions on inspections are made. The information gained during the lifetime is taken into account such that the inspection times are sequently updated.

In this paper, focus is on the use of Bayesian networks for risk-based inspection planning. This paper presents two different approaches to risk-based inspection planning where Bayesian networks are used. An application example is used to demonstrate and compare the methods.

\section{RISK-BASED DECISION MAKING}

Wind turbine components are different from most civil engineering structures in the sense that human 


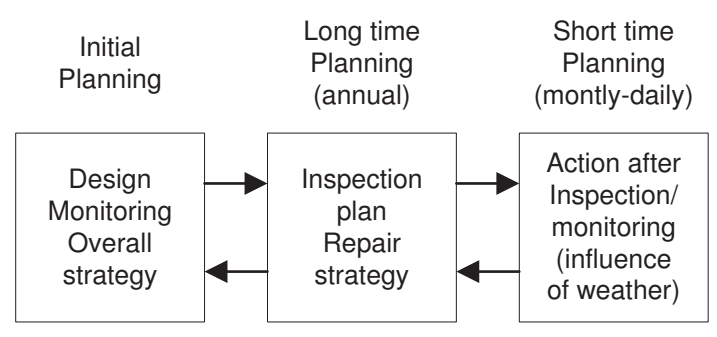

Figure 1: Time levels for O\&M planning.

lives are normally not in danger if something fails. Further, the wind turbine control system implies that energy harvesting and loads are controlled in order to maximize the energy production with 'reasonable loads'. Failure of large structural components such as blades, tower, and foundation could be dangerous for humans even though no one would normally get hurt in the case of offshore wind turbines. This means that operation and maintenance for most components can be planned from a purely risk-based approach where only costs are used in the calculations. That is initial costs, cost of monitoring, inspections, and preventive and corrective repairs, including lost production, which is normally measured through the availability of the wind turbine. The decisions should be made such that the sum of these costs is minimized.

\subsection{Formulation of decision problem}

The decisions to include in the decision problem are in principle all decisions that will influence the total costs of O\&M. In the planning phase, there are decisions regarding the design, monitoring equipment, and also the overall strategy for inspections and repairs, even though this can be changed later if appropriate. During the lifetime, decisions should be made on when, what, and how to inspect and repair, and how to handle corrective repairs. In general, the number of decision alternatives are multi dimensional and continuous, and the unreduced problem is naturally intractable.

These decisions can again be handled on different levels/different time horizons, as shown in figure 1. This paper focuses on the long time planning, i.e. which years to make inspections. Monthly planning could be relevant for components with more rapidly growing damages. In order to make short time planning, i.e. what day an inspection/repair should be performed the weather and resources (vessels/crews/spare parts) should be included. The weather is an important factor for O\&M for offshore wind turbines, as is it not possible to perform inspections and repairs in harsh weather conditions. Before an action is made, it is necessary to wait for sufficiently calm weather, and to assess the risk of the weather getting worse during the interference.

In this paper, we limit ourselves to a problem where one inspection method and one repair method is possible. Only components with failure modes that can be described as deterioration are considered. The inspections are imperfect, and the probability of detecting a damage can be described using a PoD-curve (Probability of Detection), where it is in general easier to detect larger damages.

The simplified problem can then be formulated as a Bayesian pre-posterior decision problem, as illustrated in figure 2. Each year (or another reference time, if needed) a decision should be made whether to make an inspection or not. The outcome of the inspections is then the premise for the decision on whether to make a repair. A decision rule could be formulated in different ways; for example, the decision rule could state that repairs should be performed for damage sizes above a certain threshold. Here, we apply the most simple repair decision rule, where detected damages are always repaired, and repaired components behave like new components. During the following year, until a new decision on inspection should be made, the component might or might not fail.

\subsection{Solving the decision problem}

There exist two different approaches for the classical pre-posterior decision analysis. The first approach is the normal form analysis, where the expected costs are calculated for each branch of the decision tree. The other approach is the extensive form analysis, where a decision rule should be formulated for each decision, for each combination of all past decisions, inspection results, and failures, i.e. all available knowledge.

For inspection planning, there is a time step for each year, and this complicates things further, as the decisions the second year also depend on the decisions the first year. For the problem illustrated in figure 2, the number of different branches, for which the probability and cost should be calculated, is $6^{20}=$ $3.66 \cdot 10^{15}$, which makes the decision problem intractable to solve in this way. Instead, it is necessary to find an approximate solution to the decision problem.

For risk-based planning, different approaches have been suggested where only one optimization parameter is used to find the inspection times. The most simple method uses a constant time interval between inspections. Another approach is to choose a threshold value of the failure probability, and schedule inspections just before this value is reached. Straub (2004) concluded that these two approaches gave optimal values with expected total costs close to each other. However, the inspection plans were in both methods scheduled from the beginning where only prior knowledge was available. In this paper, Bayesian networks are used to take indirect information from inspections during the lifetime into account, and two different approaches are used to revise the inspection plans each year assuming a set of observations through the lifetime. 


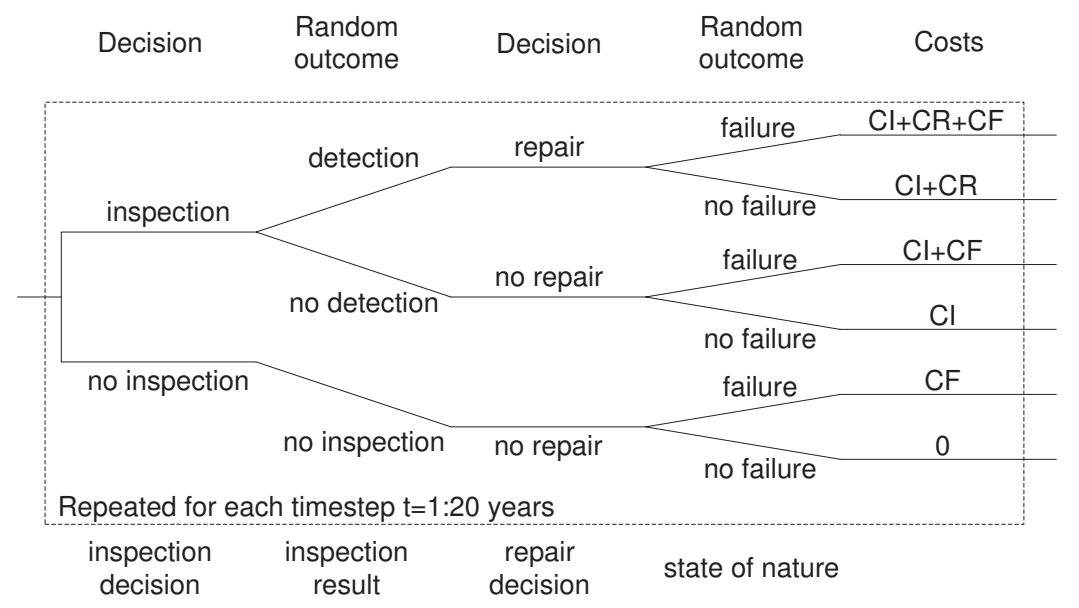

Figure 2: Decision tree for planning of repairs. CI: cost of inspection, CR: cost of repair, CF: cost of failure.

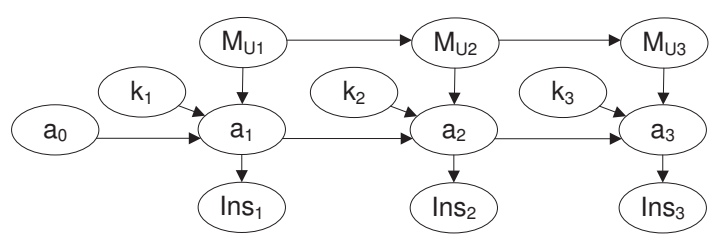

Figure 3: Bayesian network for modeling of deterioration shown for three time steps.

\section{BAYESIAN NETWORKS}

Bayesian networks were originally developed in computer science, and can be used to calculate probabilities, and to update these probabilities using Bayes rule, whenever new information becomes available. A throughout introduction is beyond the scope of this paper, and reference is given to Jensen and Nielsen (2007), where an introduction can be found.

For modeling of deterioration processes, Dynamic Bayesian networks can be used, where there is one time slice for each time step. An example is shown in figure 3 , where $a_{i}$ is the damage size, $M_{U i}$ is a time-invariant parameter, and $k_{i}$ is a time-variant parameter. The node $I n s_{i}$ models the outcome of an inspection. If an inspection is performed, the node $I n s_{i}$ will receive evidence, and the evidence will propagate through the network and update the distributions of the other variables.

In this section, the two approaches to risk-based inspection planning are described. In the first method, a threshold for the failure probability is used to decide when inspections are performed. The second method uses a Limited Memory Influence Diagram (LIMID).

\subsection{Threshold approach}

For the threshold approach, a threshold value for the failure probability is set from the beginning. The threshold value should be chosen such that the expected costs are minimized; the optimization procedure is explained later. Each year, the probability of failure is calculated for the following year. If this value exceeds the threshold value, an inspection is performed. If a damage is detected, it is repaired, which means that the failure probability is reduced. If, on the other hand, no damage is detected, it is less likely that a large damage is present, and the probability of failure will also be reduced. The Bayesian network for this approach is shown in figure 4. The node $F_{i}$ have the two states 'one' representing 'failure' and 'zero' representing 'no failure'. The node $D_{i n s, i}$ is a decision node with the states 'no inspection' and 'inspection'. The node Ins $s_{i}$ have the states 'no inspection', 'detection', and 'no detection', and is in the state 'no inspection' if the node $D_{i n s, i}$ is that as well. The $R e p_{i}$ node is in the state 'repair', if the inspection node is in the state 'detection', and else it is in the state 'no repair'. This node is actually not necessary, as the inspection node could have been a direct parent of the node $a_{i}$, representing the damage size at the next time step, instead. The chosen model, however, reduces the size of the table for the conditional probability distribution for $a_{i}$.

One could wonder why there is no link from the failure node $F_{i}$ to the inspection node $D_{\text {ins }, i}$, as it is determined based on the failure probability, whether an inspection should be performed. However, it should be noted that the failure node have the states 'failure' and 'no failure' thus it does not directly represent the failure probability. The failure probability can easily be calculated as the mean of the failure node, but this information cannot be used in the network to implement the decision rule directly. Instead the network should be used in the following way:

For each year $i$ :

- Enter 'failure' or 'no failure' in node $F_{i}$ depending on whether a failure has happened during the last year, and propagate the evidence in the network.

- If the expected value of $F_{i+1}$ (the probability of failure within a year) is larger than the threshold value of the failure probability: 


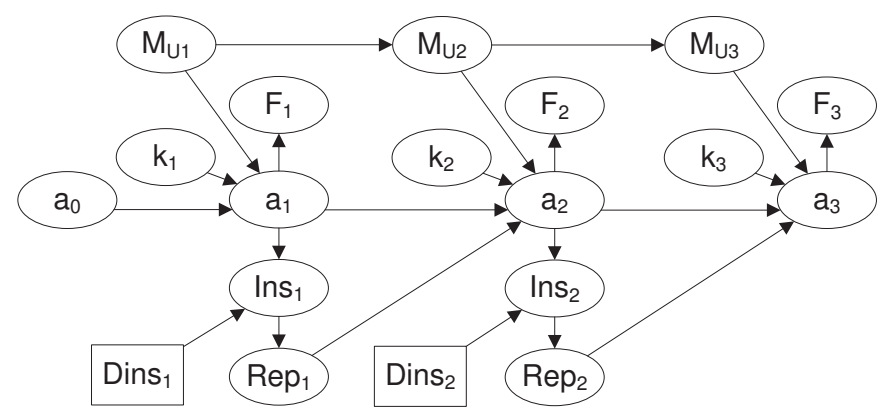

Figure 4: Bayesian network for threshold approach.

- Enter 'inspection' in node $D_{\text {ins }, i}$.

- Perform the inspection, and repair the component if a damage is detected.

- Enter 'no detection', or 'detection' in node Ins $s_{i}$ depending on the outcome of the inspection.

- Else:

- Enter 'no inspection' in node $D_{i n s, i}$.

The threshold value of the failure probability should be chosen such that the expected costs are minimized. In order to find the optimal value of the failure probability, Monte Carlo sampling is performed for different values. The procedure for the Monte Carlo sampling is as follows:

- Simulate outcome of $M_{U}$ and $a_{0}$.

- For each year $i$ :

- Simulate outcome of $k_{i}$, and calculate $a_{i}$ based on $a_{i-1}$.

- Enter 'failure' or 'no failure' in node $F_{i}$ depending on whether the calculated $a_{i}$ is larger that the failure limit, and propagate the evidence in the network.

- If the expected value of $F_{i+1}$ (the probability of failure within a year) is larger than the threshold value of the failure probability:

* Enter 'inspection' in node $D_{i n s, i}$.

* Simulate outcome of the inspection using the PoD-curve.

* If the outcome is 'detection' a repair is made: Simulate new $a_{i}$ from the distribution of $a_{0}$.

* Enter 'no detection', or 'detection' in node Ins $_{i}$ depending on the outcome of the inspection.

- Else:

* Enter 'no inspection' in node $D_{i n s, i}$.

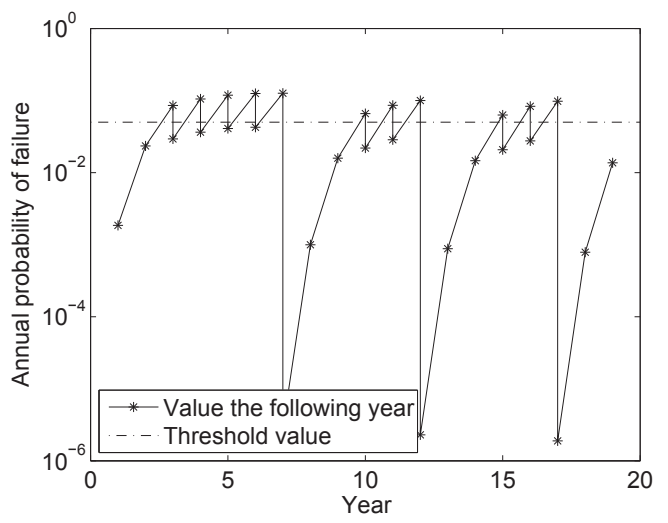

Figure 5: Annual failure probability before and after inspections (when an inspection is performed). The threshold value is 0.05 .

The number of inspections, repairs, and failures are logged for each simulation, such that expected values and confidence intervals can be calculated. When appropriate confidence intervals are obtained, the expected costs are found by multiplying the specific costs with the mean number of inspections, repairs, and failures. The procedure is repeated for different threshold values, and the value that yields minimum total expected costs is the optimal value, and should be used.

Figure 5 shows the annual probability of failure for a simulated set of observations as explained above. The value of the probability of failure is shown for each year both before any inspection is performed, and after inspection and possible repair is performed. In the given example, three repairs are made, which can be seen as large drops in the probability of failure. The small drops are caused by inspections.

\subsection{LIMID approach}

An influence diagram is an alternative graphical representation of the decision problem in figure 2. In addition to the probability nodes in Bayesian networks, influence diagrams also have decision nodes and cost nodes. The cost nodes that are relevant for this problem are nodes describing the cost of inspections, $C I_{i}$, repairs, $C R_{i}$, and failures, $C F_{i}$. Because an explicit decision rule is used for decisions on repairs, only decision nodes representing decisions on inspections 
are used here. In principle, each decision node should have a policy table that depends on the state of all past nodes, which makes the problem intractable. Instead the Limited Memory Influence Diagram (LIMID) is used, where the policies only depend on the nodes that are direct parents of the decision nodes, see (Lauritzen and Nilsson 2001). The optimal decisions are found using the Single Policy Updating (SPU) algorithm. The algorithm starts with initial policy tables, and calculates the expected costs for the given set of decisions. The policy tables are changed one at a time until smaller expected total costs cannot be obtained by changing only one policy. Dependent on the problem, the algorithm might therefore get stuck at a local minimum that is not the global minimum. However, wise choice of parents for the decision nodes can reduce this problem. For this application, the previous inspection result is set as parent, as this result highly influences the decision the following year. The network for the LIMID approach is shown in figure 6 .

Because the policy tables only include the parents, it is necessary to update the policies whenever new evidence has been received in order to make the optimal decisions. This is not taken into account when the expected costs are calculated directly from the network, as it is assumed that the policies found in year zero are used. The correct expected costs, if the network is updated during the lifetime, will normally be higher than the one estimated directly from the network. Therefore, Monte Carlo sampling is also used here to calculate the correct expected costs for this strategy. The procedure is similar to the one used for the threshold approach, but the decision on inspection is found by updating the policies after it has been entered whether failure has occurred.

\section{EXAMPLE}

In this section, the methods described above are demonstrated through an illustrative application example. First, a damage model with a measurable damage size should be established based on available data and physical models for the current deterioration mode. For hot spots designed for fatigue, an FMmodel can be established based on the $\mathrm{SN}$-curves used for the design, see (Straub 2004). If data is available from inspections, Bayesian networks can be used for learning the model from data. The establishment of an accurate model is important, but it is not treated further here, as it is beyond the scope of this paper. Instead, a damage model based on Paris law is chosen with a failure rate typical for some wind turbine components, 0.1/year. Another damage model could be used instead and the same methods could still be used.

In Paris law the damage growth per stress cycle, $d a / d N$, can be found as:

$\frac{d a}{d N}=C \cdot \Delta K^{m}$

\begin{tabular}{llll}
\hline Variable & Distribution & Mean & $\mathrm{CoV}$ \\
\hline$m$ & Deterministic & 2.1 & - \\
$C$ & Deterministic & $8.5 \cdot 10^{-10}$ & - \\
$B$ & Deterministic & 0.66 & - \\
$Y$ & Deterministic & 1 & - \\
$N$ & Deterministic & $10^{6} /$ year & - \\
$a_{0}$ & Exponential & $0.02 \mathrm{~mm}$ & $100 \%$ \\
$k_{i}$ & Normal & $5.35 \mathrm{MPa}$ & $10 \%$ \\
$M_{U i}$ & Normal & 1 & $18 \%$ \\
$\lambda$ & Deterministic & 0.4 & - \\
\hline
\end{tabular}

Table 1: Distributions for parameters used in the model.

where $\Delta K$ is the stress intensity factor range, and $C$ and $m$ are model parameters. If it is assumed that the stress ranges are Weibull distributed on a yearly basis with scale parameter $k_{i}$, and shape parameter $B$, the following expression can be derived for the damage size at time step $i$ based on the damage size on time step $i-1$, see e.g. (Friis-Hansen 2000):

$a_{i}=\left(a_{i-1}^{\frac{2-m}{2}}+\Delta K M_{U i} k_{i}^{m}\right)^{\frac{2}{2-m}}$

where the stress intensity factor range, $\Delta K$, is found using:

$\Delta K=C N \Gamma\left(1+\frac{m}{B}\right) Y^{m} \pi^{\frac{m}{2}}\left(1-\frac{m}{2}\right)$

where $Y$ is a geometry parameter, $N$ is the number of stress cycles per year, and $M_{U i}$ is a parameter that models the model uncertainty.

The probability of detecting a damage is assumed to increase with the size of the damage, and is described by the PoD-curve:

$\operatorname{PoD}(a)=1-\exp (-a / \lambda)$

where $\lambda$ is the expected value of the size of detectable damages. The values and distributions for the parameters are shown in table 1.

The Bayesian networks are build in the program Hugin, where exact inference can be made for networks with discrete distributions. The nodes $k_{i}$, and $M_{U i}$ have a range from minus to plus infinity, and have each been discretized in 10 states. They are truncated at plus/minus three standard deviations, and the internal states are of equal size. The node $a_{i}$ has a range from zero to infinity, and the last state goes from 1 , where failure occurs. It has 30 states, and the intervals in the interval from $10^{-4}$ to 1 are of exponentially increasing size.

The normalized specific costs used in the example are shown in table 2. No specific component is considered, but it could for example be components like main bearing, gearbox, or pitch system. The ratio between the repair and failure costs depends on, for instance, the difference in repair type for repairs performed before and after the actual failure, the expected downtime in case of failure, and the possibility to plan multiple preventive repairs together, and 


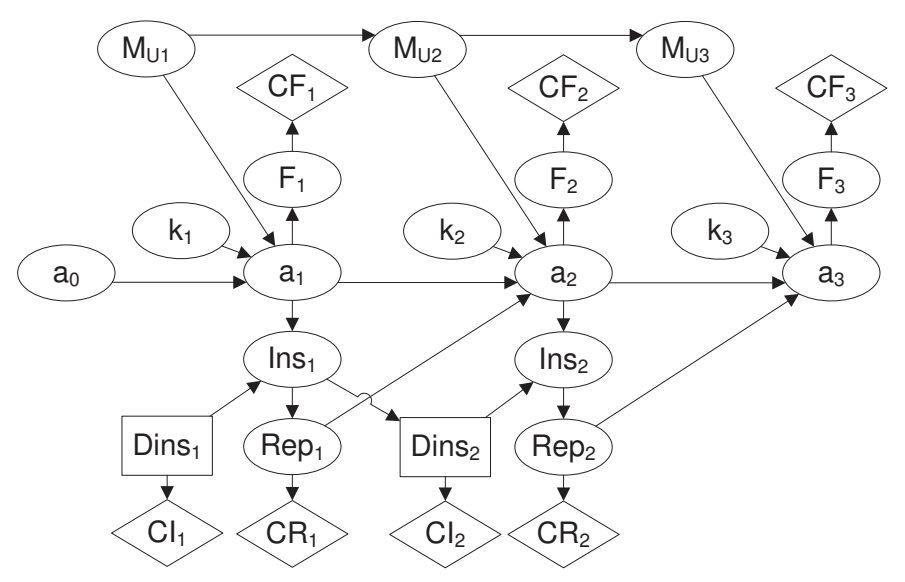

Figure 6: Bayesian network for LIMID approach.

\begin{tabular}{lll}
\hline Description & Node & Cost \\
\hline Inspection & $C I_{i}$ & 1 \\
Repair & $C R_{i}$ & 10 \\
Failure & $C F_{i}$ & 50 \\
\hline
\end{tabular}

Table 2: Costs used in the example.

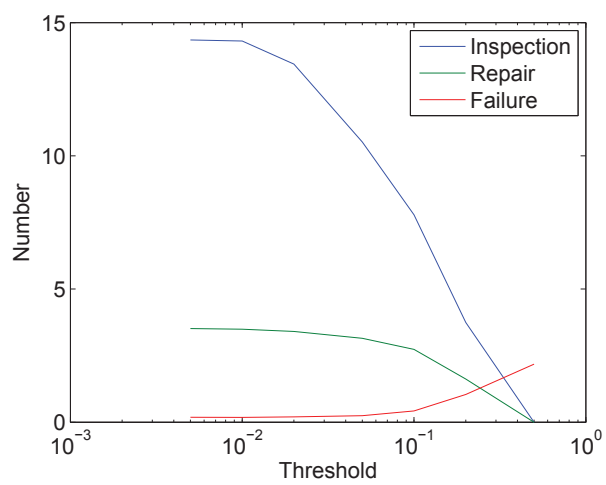

Figure 7: Number of inspections, repairs, and failures for different thresholds of the annual failure probability.

thereby reduce the total equipment costs. For failure types, where excessive indirect damage can happen in case of failure, the ratio between the repair and failure costs could be much larger.

In order to find the expected costs for the two methods and for different threshold values, Hugin's Java API has been used for building the network and performing Monte Carlo sampling over the lifetime of 20 years. For the threshold method, the mean number of inspections, repairs, and failures during the lifetime is shown in figure 7 , and the expected costs are shown in figure 8 for $10^{4}$ simulations. The optimal threshold value is 0.05 , as the total expected costs are smallest here; however, the curve is rather flat around the minimum value. This optimal value is relatively high compared to cases where safety is an issue, and the consequences of failure are larger. In the present example, the consequence of failure is relatively small, and because a smaller threshold value only slightly reduces the number of failures, it does not pay off to make the extra inspections.

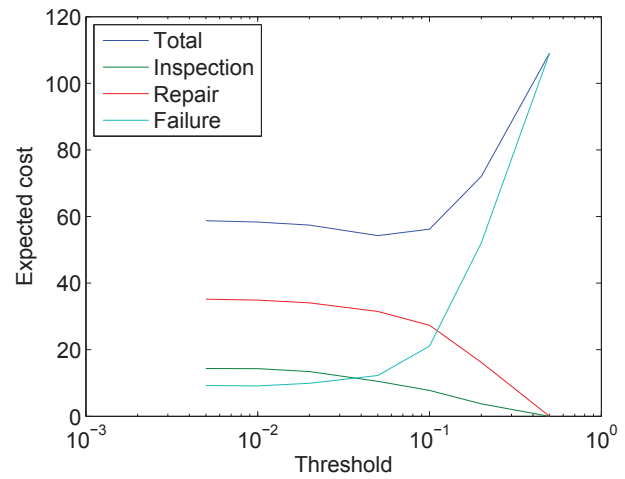

Figure 8: Expected costs for different thresholds of the annual failure probability.

The optimal value depends on the choice of specific costs, and in figure 9 the total costs are shown as function of the threshold value of the failure probability for different values of the specific costs of inspections, repairs, and failures. In general, the optimal threshold value is larger for larger relative costs of inspections or repairs. Similarly, the optimal threshold would be expected to decrease for larger failure costs. In this example, however, a double of the failure costs did not change the optimal threshold value within the used resolution, but the curve is even more flat for lower values and steep for larger values.

For the LIMID approach, the total expected cost can be found directly from the network to be 64.5. However, this is an approximation as explained in section 3.2, and the correct expected costs are found using Monte Carlo sampling to 54.0, which is significantly lower, and almost equal to the optimal value for the threshold approach. The expected costs for inspections, repairs, and failures are shown in table 3 for both the threshold approach and the LIMID approach. For the LIMID, the costs of inspections and repairs are 1.5 higher than for the threshold approach, but the costs due to failures are 1.8 lower, which gives smaller total costs. The costs, however, are very close, and the methods are considered as being equally effective. 


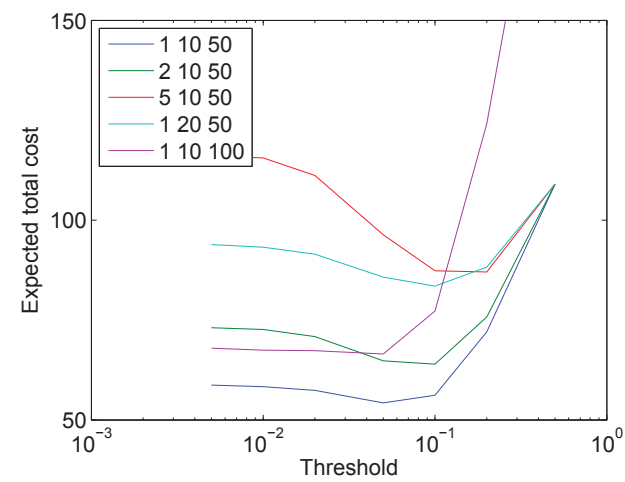

Figure 9: Total expected costs for different thresholds of the annual failure probability for different costs. The numbers refers to cost of inspections, repairs, and failures respectively.

\begin{tabular}{lllll}
\hline Case & Total & Inspection & Repair & Failure \\
\hline Threshold & 54.3 & 10.5 & 31.5 & 12.3 \\
LIMID & 54.0 & 11.6 & 31.9 & 10.5 \\
\hline
\end{tabular}

Table 3: Expected costs for threshold and LIMID approach. Costs are shown for a threshold 0.05 , which gives minimum expected costs for this strategy.

\section{CONCLUSION}

This paper presents how Bayesian networks can be used for risk-based inspection planning for offshore wind turbines. The inspection plans are updated each time new information become available from inspections, such that the optimal decisions on inspections are made using all available data. Two different methods for planning are demonstrated. In the first approach, a threshold value of the failure probability is chosen, and inspections are performed before this value is reached. The optimal threshold for the failure probability is found using Monte Carlo sampling for different thresholds. The second method uses a Limited Memory Influence Diagram (LIMID), and here the SPU (Single Policy Updating) algorithm is used to find the optimal decision with the smallest expected costs. However, the expected costs found using this method are approximations; thus Monte Carlo sampling is used to find the correct expected costs when this method is used.

The threshold method was found to give smallest expected costs for the threshold value of the annual probability of failure 0.05 for the model and costs used in the example. The expected costs were almost equal for the threshold and LIMID approach, and they are considered as being equally good. The advantage of using the LIMID method is that no optimization has to be performed initially. On the other hand, the real-time computations are simpler for the threshold approach, and for more complicated models this might be an important factor.

For instance, the model could be extended to take system effects into account by including more components and/or more wind turbines in a wind farm in the same model, and take the correlations into account.
This would probably cause the SPU algorithm to get stuck at a local minimum, and therefore the threshold approach could be better. However, not all types of correlations can be implemented easily, as the computational demands will be enormous in some cases.

Future work could also aim at including monitoring results as indirects information in the model, such that these are also taken into account when the decisions are made. This means that information with different reliability is used to update the same damage model, and Bayesian networks are efficient for this.

\section{ACKNOWLEDGEMENTS}

The work presented in this paper is part of the project "Reliability-based analysis applied for reduction of cost of energy for offshore wind turbines" supported by the Danish Council for Strategic Research, grant no. 2104-08-0014. The financial support is greatly appreciated.

\section{REFERENCES}

Faber, M. H., J. D. Sørensen, J. Tychsen, \& D. Straub (2005). Field implementation of RBI for jacket structures. Journal of Offshore Mechanics and Arctic Engineering 127(3), 220226.

Friis-Hansen, A. (2000). Bayesian networks as a decision support tool in marine application. $\mathrm{Ph}$. D. thesis, Technical University of Denmark.

Jensen, F. V. \& T. D. Nielsen (2007). Bayesian Networks and Decision Graphs. Information Science and Statistics, Springer.

Lauritzen, S. L. \& D. Nilsson (2001). Representing and solving decision problems with limited information. Management Science 47(9), 1235-1251.

Nielsen, J. \& J. Sørensen (2010). Bayesian networks as a decision tool for O\&M of offhore wind turbines. Proceedings of the 5th international ASRANet Conference.

Obdam, T., L. Rademakers, \& H. Braam (2009). Flight leader concept for wind farm load counting final report. Technical Report ECN-E-09-068.

Rademakers, L., H. Braam, T. Obdam, \& R. v.d. Pieterman (2009). Operation and maintenance cost estimator (OMCE). Technical Report ECN-E-09-037.

Raiffa, H. \& R. Schlaifer (1961). Applied statistical decision theory. Harvard University.

Straub, D. (2004). Generic approaches to risk based inspection planning for steel structures. Ph. D. thesis, Swiss Federal Institute of Technology, ETH Zurich.

Straub, D. (2009). Stochastic modeling of deterioration processes through dynamic bayesian networks. Journal of Engineering Mechanics 135(10), 1089-1099.

Straub, D. \& M. H. Faber (2006). Computational aspects of riskbased inspection planning. Computer-Aided Civil and Infrastructure Engineering 21(3), 179-192. 



\section{A.4 Paper 4}

\section{Title:}

\section{Challenges for Risk-based Maintenance Plan- ning for Offshore Wind Turbines}

\section{Authors:}

Nielsen, J. J. and J. D. Sørensen.

Conference:

21st International Offshore and Polar Engineering Conference, ISOPE 2011, Maui, Hawaii, USA. 


\section{Request for permission to include paper in thesis}

Dear ISOPE,

I attended the ISOPE-2011 conference, and had a paper included in the proceedings. I would like to ask permission to include that paper in the publication of my $\mathrm{PhD}$ thesis. The paper has the title "Challenges for Risk-based Maintenance Planning for Offshore Wind Turbines". The authors of the paper are myself and my $\mathrm{PhD}$ supervisor.

Best regards,

Jannie Nielsen

\section{RE: Request for permission to include paper in thesis}

\section{Jannie,}

Permission is granted as requested.

Best wishes,

Jin

Prof. Jin S CHUNG

ISOPE, 495 North Whisman Road, Suite 300,

Mountain View, California 94043-5711, USA

www.isope.org ; www.isope2013.org ; www.deepoceanmining.org 


\title{
Challenges for Risk-based Maintenance Planning for Offshore Wind Turbines
}

\author{
Jannie J. Nielsen and John D. Sørensen \\ Department of Civil Engineering, Aalborg University. \\ Aalborg, Denmark
}

\begin{abstract}
This paper concerns the development of a probabilistic model for riskbased maintenance planning for offshore wind turbines. A prior damage model can be combined with data to give an updated estimate of the probability of failure. For indicators, an indicator model is needed, and this paper presents how Bayesian networks can be used to learn such a model when past data is available.
\end{abstract}

\section{KEY WORDS:}

Risk-based planning; Maintenance; Bayesian Networks; indicators.

\section{INTRODUCTION}

For offshore wind turbines, many unplanned component failures happen, which results in large costs for operation \& maintenance due to corrective maintenance. In the wind energy industry, there is a high interest in reducing this number by using preventive maintenance methods. Procedures for cost optimal planning can be developed based on risk-based methods, where all relevant available information is taken into account, and can be used for updating the models successively during the lifetime, see a framework in (Sørensen 2009).

In order to make risk-based planning, it is necessary to obtain knowledge about the failure modes that are significant contributors to the overall cost of maintenance. For these failure modes, probabilistic damage models have to be developed, and the loads and damage parameters that are relevant should be included in the models. Further, models for the indicators on the damage state and loads should be developed and united in a joint model with the damage model.

Available for the establishment of this model are physical models and data from past experience, for example mean time between failures for a large number of wind turbines, and more detailed data for a more limited number of turbines, for instance SCADA data, inspection data, load measurements, information on repairs, and possibly other sensor data. This implies a challenge as the models have to be build based on indicators, and not on direct measurements of the damage size. Bayesian networks are a promising tool for this challenge, as they perform efficient Bayesian updating, and algorithms are available for learning the conditional probability distributions from data.

This paper discusses the challenges in developing such a model from limited data available, a generic model is presented, and an illustrative example is shown to illustrate the capabilities of learning parameters in models using Bayesian networks.

\section{DECISION PROBLEMS}

Decision problems for maintenance planning typically concern when/how to maintain or repair and when/how to inspect and monitor. In principle, infinitely many possibilities exist, and therefore, the problems need to be reduced such that only the most important issues are considered. Which issues are most important highly depends on the considered component. Wind turbines consist of electrical, mechanical, and structural components, and these different component types differ on several important points, for example:

- Failure rate

- Failure type

- Indicators available

- Damage measurement data (crack length, corrosion depth)

- $\quad$ Possibilities for maintenance/repair

Failure rates are typically relatively large for electrical components, smaller for mechanical components, and much smaller for structural components, see (Faulstich, Hahn, Lyding, and Tavner 2009). On the contrary, the downtime and cost associated with a failure is larger for the structural and some mechanical components. The failure type and the presence of good indicators are very important when preventive maintenance is considered. Preventive risk-based maintenance is mainly applicable for components where failures are caused by deterioration, which is the case for many structural and mechanical components. Also the size of the components and the severity of the failures are important for the cost of repairs. For large components, expensive equipment is necessary for repair and the downtime can be long, especially if the wind turbines are offshore. 
When risk-based methods are used for planning of preventive maintenance, it is important to identify where something can be saved compared to the case with only corrective maintenance. This depends on the downtime, unit repair cost, and mobilization cost.

In cases, where repairs are very cheap, downtime can be the cost driver, as harsh weather can prevent repairs from being possible. In such cases, the costs can be reduced by using preventive maintenance instead of corrective.

For more expensive repairs, the downtime may not have a significant influence on the overall costs. However, other conditions can still make preventive maintenance viable. This applies to cases, where total replacements are necessary if failure happens, but where cheaper repairs can be made, if damages are detected prior to failure.

For some large components, neither the downtime or unit repair costs are significant compared to the mobilization costs for the needed vessels, and the mobilization costs are the same for preventive and corrective repairs. Consequently, preventive maintenance is not viable for a single wind turbine, but it can be viable when an entire wind farm is considered. If one wind turbine fails, more wind turbines can be repaired at the same time at a low additional cost.

In all the presented cases, the preventive effort should be balanced, such that the total expected costs are minimized. Risk-based methods can be applied to find the optimal strategy. In principle, preventive repairs should be made, if the total expected costs during the remaining lifetime are reduced by making it. This can be modeled and solved by a Bayesian pre-posterior decision problem. The problem can be simplified by introducing a decision rule for when preventive maintenance should be performed, for example a threshold value for the probability of failure. The threshold should then be determined such that the expected total costs during the lifetime are minimized.

\section{PROBABILISTIC MODEL}

In order to solve the decision problems discussed above, a probabilistic damage model should be established. The damage model can be established based on the design, but large uncertainties are present. The uncertainties can be divided in two different types, aleatory and epistemic. The aleatory uncertainty comes from the physical variability, and cannot be reduced. The epistemic uncertainties comprise model, statistical, and measurement uncertainties, and they can be reduced. After realization of the variables with aleatory uncertainty, the uncertainty becomes epistemic, and can then be reduced.

In general, the better the model, the smaller the uncertainties, the better basis for decision making, and the smaller the costs. Therefore, the damage model should be improved using the data available. Data can be used to reduce the statistical uncertainties and update the statistical description of model uncertainties. Some data represent the cause of the degradation, such as load measurements, and some data represent the effect of the degradation; they can be either direct measurements of the damage or indicators of the size of the damage.

\section{Prior estimate}

Deterioration can often be described using fracture mechanical models, where the damage size is explicitly modeled. Often, the design models do not include a measurable damage size, but they can be used to establish a connection to the reliability level required in design codes. In the fracture mechanical models, the uncertainties are included by modeling the parameters as stochastic variables, and the value of some of the variables can be calibrated to give the requested reliability level.

Generally, the damage size at time $t$ can be specified as a deterministic function $g$ of the initial damage size $D_{0}$, some load variables $A$, and some strength variables $C$, which can be either time-variant or timeinvariant:

$$
D(t)=g\left(D_{0}, A(t), C(t)\right)
$$

In a probabilistic model, the variables $A, C$, and $D_{0}$ are stochastic variables. Using this model, the probability distribution, $f(D)$, for the damage size can be found at an arbitrary time, and together with a limit state equation, the probability of failure can then be estimated.

\section{Updated estimate}

To get an updated estimate on the probability of failure, the prior damage model should be combined with data, as illustrated in Fig. 1. In the simplest case, the damage size is directly observable. At the time where observations have been made, the damage size is then known with certainty. The distributions for the variables $A, \mathrm{C}$, and $D_{0}$ can then be updated, and the predictions for the future are updated. Similarly, if the load is directly measurable, the load variables for past realizations are known with certainty, and the predictions can be updated.

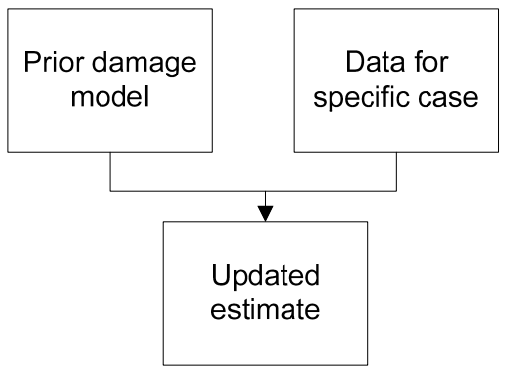

Fig. 1. Updating of the estimate.

In a more general case, where the observations are from indicators, an indicator model should be given. The indicator model gives the outcome for the indicator conditioned on the damage size:

$$
f_{I \mid D}(I(t) \mid D(t))
$$

An example of such a model is a probability of detection (PoD) curve. There two outcomes are possible, detection or no detection, and the probability for each depends on the damage size. 
Bayesian updating can be used to find the probability distribution for the damage size given the indicator data $\hat{\mathrm{I}}$ :

$$
f_{D \mid I}(D \mid \hat{I}) \propto f_{D}(D) f_{I \mid D}(\hat{I} \mid D)
$$

\section{Updated models}

In the cases described above, the uncertainties for the parameters in the models are updated, and thereby, the predictions are updated. But the models, given by equation (1) and (2), are not changed. If data is available for a number of 'sister' wind turbines, these data can be used to update the models, as illustrated in Fig. 2. The data could comprise load measurements, observations from indicators, and information about failures. This case will be treated later in the paper.

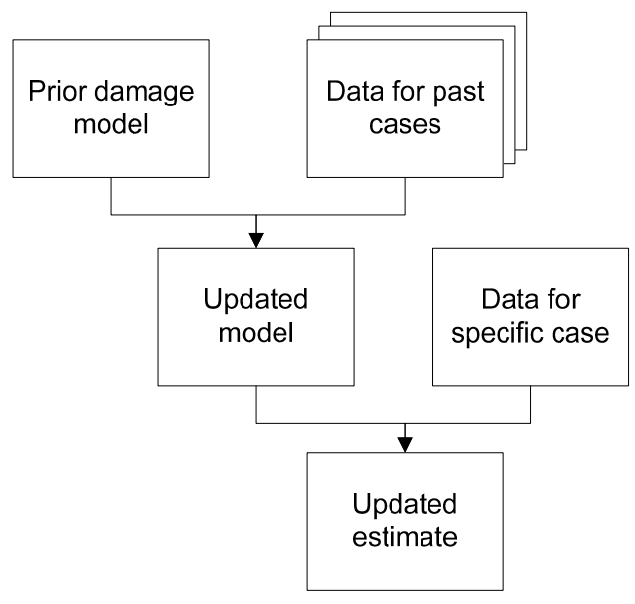

Fig. 2. Updating of the model and the estimate.

\section{AVAILABLE DATA}

This section discusses the types of available data, and the challenges being present. For the public, limited data is available from databases such as WMEP, see (Spinato, Tavner, van Bussel, Koutoulakos 2009) and (Faulstich, Hahn, Lyding, and Tavner 2009). These databases contain failure rates and downtimes for a large number of different wind turbine components for different models and manufacturers.

On the other hand, wind turbine manufactures and owners, generally, have much more detailed data for their own wind turbines, such as:

- $\quad$ SCADA data

- Measurement data (e.g. vibrations and loads)

- Logs of alarms and failures

- Inspection reports

- $\quad$ Repair and maintenance reports
The SCADA data is acquired for all wind turbines every ten minutes, and consist of statistical data for, for example, pitch angle, jaw angle, temperatures, wind speed, and production. For modern wind turbines, the SCADA data can consist of more than hundred channels. Measurement data can for example be vibration time series recorded once a day, when the turbine is within prespecified operational conditions.

The inspection, repair, and maintenance reports can either be electronic or hand-written, and the quality differs. If precise procedures are not specified for the technicians, it can be hard to get full use of the information. Inspection reports can contain direct measurements of the damage size.

The SCADA and measurement data comprise large amounts of data. Therefore, the first step is to select the relevant data only, and process the data to usable numbers, as it is not feasible to make a model where everything is used.

\section{Indicator data}

Some SCADA data are indicators for the condition of components, for example temperature and vibration measurements. For healthy components the measurements are within a certain range, but in the case of developing damages the readings increase. An alarm threshold can be set for the readings based on past data. Generally, there is a natural variability for a healthy component, and for some indicators, the readings are dependent on other conditions. For example, the main shaft temperature could be correlated with the rotational speed of the shaft, and a high temperature would be unusual for low speeds, but normal for high speeds. In that case, the alarm threshold could be dependent on the shaft speed. Generally, the alarm levels should be set based on analyses of past data and physical understanding, and information on the presence of alarms can be used to update the estimate.

One of the challenges in using the data for updating of a general model is that the data is for various wind turbine models and various environmental conditions. Therefore, the data cannot be considered as being from the same population. When data is used to update a model, it is important to consider whether the data is directly applicable, or maybe applicable given some conditions that are included in the model. For example, if two equal wind turbines are placed two different places, the data for one wind turbine can be applied to the other, if the environmental conditions are included in the model.

\section{Load data}

For deterioration such as fatigue, the load cycles drives the damage development. If the load is measured continuously and a model is established to estimate the load effect driving the damage, rainflow counting can be performed to give a histogram for the load cycles.

If the load is not directly measured, it can be estimated based on the standard SCADA data. Obdam, Rademakers, and Braam (2009) have used neural networks to estimate the load from SCADA data. Load measurements and SCADA data from a few wind turbines was used to train the network, and the load was then estimated for other wind turbines in the wind farm. In the neural network approach, no theoretical model for the relationship between the data and load effect is included. 
Another approach is to combine the theoretic models with the data. In the design of a wind turbine, an aeroelastic code such HawC is used to estimate the load cycles conditioned on the operational condition, wind speed, and turbulence intensity, as illustrated in Fig. 3. The SCADA data include indicators of these values, and thus the load can be estimated by combining the results from the earoelastic model with the SCADA data. A model can be established to calculate the equivalent number of load cycles conditioned on SCADA data measurements, such as wind speed, yaw angle, and pitch angle. The turbulence intensity depends on the wind speed and the presence of wakes from other turbines. The existence of wakes can be estimated based on the yaw angle together with relative position to other wind turbines. To assess the operational state, SCADA data such as pitch angle, yaw angle, and wind direction can be used.

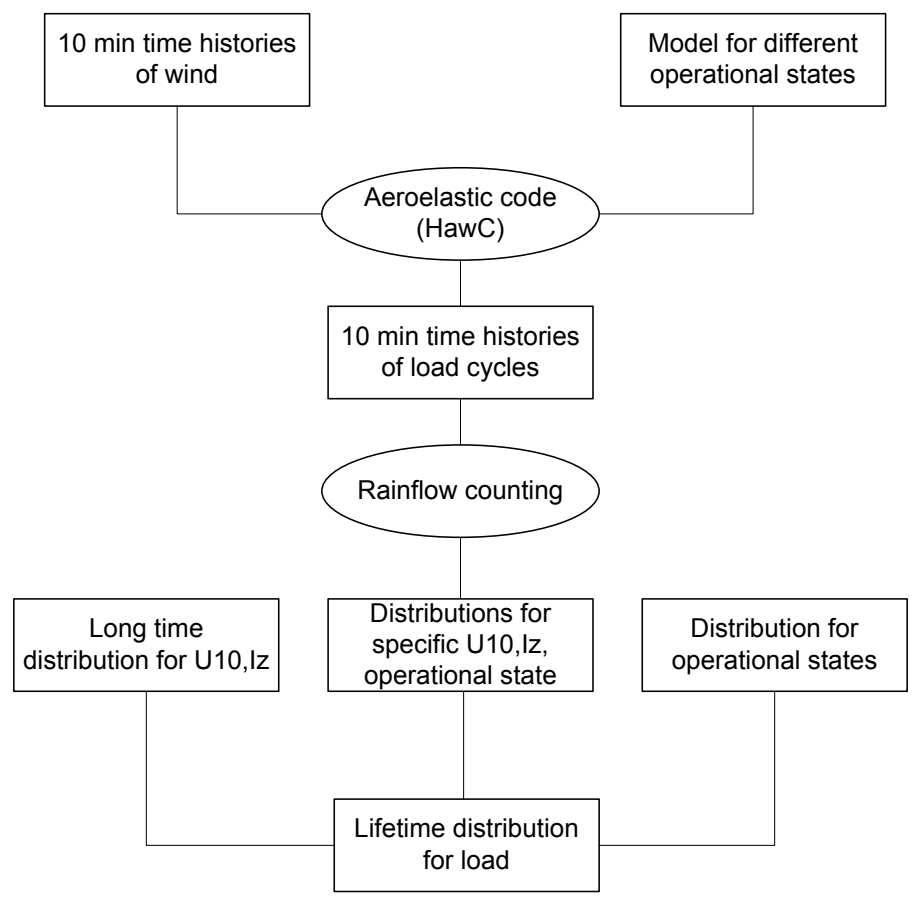

Fig. 3. Model for calculation of the distribution for load effect cycles.

\section{BAYESIAN NETWORK MODELS}

The updating of estimators and models described earlier can only be performed analytically for simple cases. Generally, closed form solutions cannot be found, and approximations are necessary. Furthermore, complicated models can be hard to assess. Bayesian networks can be useful for modeling of such problems, and various algorithms for updating exist. This paper only gives a brief introduction to Bayesian networks, for elaboration see (Jensen and Nielsen 2007).

Bayesian networks are graphical models consisting of nodes representing stochastic variables and links representing causal relationships between the variables. Further, a probability distribution has to be specified for each node, conditioned on the parental nodes. Parental nodes are the nodes with links pointing from them to the given node. When data is received for some variable in the network, the data can be propagated based on Bayes rule. Hereby, the posterior distributions for the remaining nodes can be found conditioned on the data.
For networks with discrete nodes only, efficient updating can be performed exact using, for example, the junction tree algorithm. For continuous nodes an approximation is needed. An option is to discretize all continuous nodes, thereby the algorithms for discrete nodes can be used. Alternatively, Markov Chain Monte Carlo methods, such as the Gibbs sampler, can be used. This paper will focus on discrete models in the following.

\section{Prior estimate}

For the application deterioration modeling, dynamic Bayesian networks can be used, see (Straub 2009). Dynamic Bayesian networks consist of a number of equal time slices that are connected to the neighboring slices only. This implies that the damage size at one time step only depends on the damage size at the previous time step and some other parameters included in the model. Each slice consists of a node representing the damage size, nodes for time variant and time-invariant parameters, and nodes for the indicators. A general model is shown in Fig. 4.

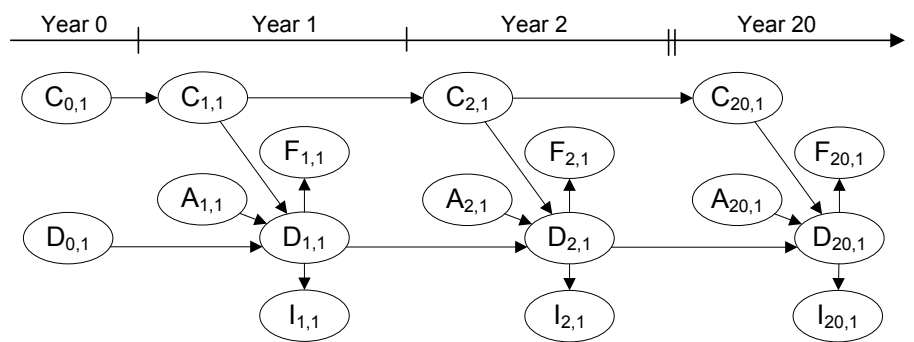

Fig. 4. Bayesian network for updating of the probability of failure.

The nodes in Fig. 4 are generally continuous. For the nodes without parents, the marginal probability distributions should be specified and discretized. For the time invariant parameters, a node is included in each time slice such that each slice is only connected to the neighbors. The node in the first slice has the marginal distribution, for the remaining slices the conditional distribution is the identity matrix.

For the node representing the damage size the conditional probability distribution should be specified:

$$
f_{D}\left(D_{i} \mid D_{i-1}, A_{i}, C_{i}\right)
$$

In the continuous case, the damage model will often be given as a deterministic function of the variables. But when the continuous distributions are discretized, randomness is introduced in the conditional probability distribution. This distribution can be found using sampling.

The step length should be chosen with the computational time and decision problem in mind. For some problems a time step of one year could be appropriate, for other problems a month or less might be necessary. 
The network shown in Fig. 4 can be used to calculate the marginal probability distributions for the damage size. If one of the states of the damage node represents failure, the probability of failure can be found. The model can also be used directly to update the estimate if the damage size is directly observable. Similarly, if one of the time dependant variables $A$ represents the load, and the load is directly observable, the same model can be used. The procedure for this is that evidence is entered into the network and propagated. The model then gives the posterior distributions for the damage size and the probability of failure.

\section{Updated estimate and parameters}

For indicators, nodes should be included in each time slice, as shown in Fig. 4. The states of the nodes should correspond to the possible outcomes for the indicator. For SCADA data where alarm levels are set, the states could simply be alarm and no alarm. In principle, the readings for the SCADA could also be used as continuous data, but here the difference in time steps for the data and the Bayesian network could be a problem. The conditional probability distribution for indicator nodes could be specified as:

$$
f_{I}\left(I_{i} \mid D_{i}\right)
$$

Alternatively, if the indicator model is uncertain, parameters can be included in the model. If data is available from past realizations, the parameters included in the model can be updated by including a string for each wind turbine. Processed data for all available cases can then be entered into the network, and the distributions can be updated. This case is further explained in the application example.

\section{Updated estimate of model without parameters}

In the situation described above, data is used to update the posterior distributions, but the conditional distributions are not changed. Updating of distributions can be relevant if large uncertainties are present in the model and large amounts of data are available, for instance, data for all turbines in a wind farm. Updating of these distributions based on data is called learning, and will be dealt with in the following.

The principle for learning is that the most likely probability distributions are found, conditioned the dataset. In the simple situation, where all variables are directly observed in all cases, maximum likelihood estimation can be used. For each conditional probability distribution, the number of data counts for each state given each parental configuration is found and divided by the total number having that parental configuration. Alternatively, a Bayesian approach can be used, where a prior distribution is taken into account.

When all variables are directly observed in all cases, the dataset is called complete. If some variables are not observed in some or all cases, the dataset is incomplete. In the case of incomplete data the Expectation-Maximization (EM) algorithm can be used see (Jensen and Nielsen 2007). The EM algorithm is iterative, and each iteration consists of two steps, the expectation step and the maximization step. In the expectation step, the dataset is completed by finding the probability distributions for the non-observed variables given the data. In this calculation, the conditional probability distributions are used. In the maximization step, the completed dataset is used to find the most likely conditional probability distributions. The procedure is repeated until convergence is reached.
For the present application, a dynamic Bayesian network is used. Here the conditional probability distributions are equal in all time slices, and this restrain should be applied during the learning procedure. Further, it can be chosen to learn just some of the distributions.

\section{ILLUSTRATIVE EXAMPLE}

This example shows how data can be used to estimate a parameter in an indicator model. A component is considered where fatigue failure is the design driver, and where the model and uncertainties for the damage development are known. SCADA data measurements are available from past experience together with the information about failures. We assume that no preventive maintenance methods were used in the past cases.

The aim is to start using preventive maintenance for a similar future case, and use SCADA data to update the estimate. Therefore the relation between the SCADA data and damage size is needed. We assume that one of the SCADA channels are known to be an indicator for the damage size, and based on the past data a reasonable alarm threshold for the SCADA channel can be set. The probability of reaching the alarm threshold increases with increasing damage size, and is described by a PoD curve. This example shows how to estimate the parameter in the PoD curve based on the past SCADA data.

\section{Damage model}

For a component where fatigue failure is the design driver, the deterioration can often be described using Paris law, where the damage growth per stress cycle is given by:

$$
\frac{d D}{d N}=C \cdot \Delta K^{m}
$$

where $C$ and $m$ are model parameters, and $\Delta K$ is the stress intensity factor range. In the case where the stress ranges follow a Weibull distribution on a yearly basis with scale parameter $A$ and shape parameter $B$, the following solution can be found to the differential equation:

$$
D_{i}=\left(D_{i-1}^{\frac{2-m}{2-m}}+\Delta K M_{U} A_{i}^{m}\right)^{\frac{2}{2-m}}
$$

$M_{U}$ models the time-invariant model uncertainty, and $\Delta K$ is given by:

$$
\Delta K=C N \Gamma\left(1+\frac{m}{B}\right) Y^{m} \pi^{\frac{m}{2}}\left(1-\frac{m}{2}\right)
$$

where $Y$ is a geometry factor, $N$ is the number of load cycles per time step, and $\Gamma($.$) is the gamma function. The parameters have the values$ given in table 1 , where $D_{0}$ is the initial damage size.

For the indicator, a probability of detection $(\mathrm{PoD})$ curve is used with the form:

$$
\operatorname{PoD}(D)=1-\exp (-D / \lambda)
$$

The parameter $\lambda$ represents the expected detectable damage size. The PoD curve is shown in Fig. 5 for different values of $\lambda$. For small values of $\lambda$, the curve is steep, and damages are detected at an early state. For larger values, even large damages are not easily detected. 
Table 1. Distributions for parameters in the damage model.

\begin{tabular}{|l|l|l|l|}
\hline Variable & \multicolumn{1}{c|}{ Distribution } & \multicolumn{1}{c|}{ Mean } & \multicolumn{1}{c|}{ CoV } \\
\hline$m$ & Deterministic & 2.1 & - \\
\hline$C$ & Deterministic & $8.5 \mathrm{e}-10$ & - \\
\hline$B$ & Deterministic & 0.66 & - \\
\hline$Y$ & Deterministic & 1 & - \\
\hline$N$ & Deterministic & $10 \mathrm{e} 6$ & - \\
\hline$D_{0}$ & Exponential & 0.02 & 1 \\
\hline$A$ & Normal & 5.35 & 0.1 \\
\hline$M_{U}$ & Normal & 1 & 0.18 \\
\hline
\end{tabular}

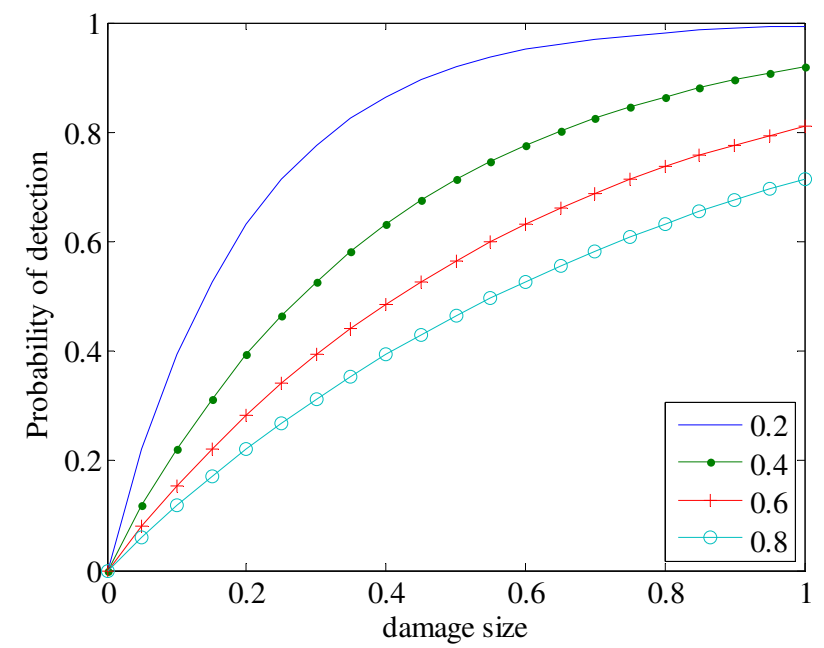

Fig. 5. Probability of detection curve for different values of $\lambda$.

The Bayesian network for the problem is shown in Fig. 6. All the nodes are discretized in intervals as given in table 2. The intervals for the nodes representing the damage size are exponentially increasing with the damage size, whereas the other nodes have uniform interval sizes. The nodes $D_{0, j}, A_{i, j}$, and $M_{U 0, j}$ have the distributions given in table 1 discretized into the given intervals. For $D_{i, j}$ the conditional probability distribution is found using equation 7 using Monte Carlo sampling. In this example it is assumed that no prior knowledge is available about $\lambda$. Therefore, a non informative prior should be used, and here a uniform distribution is chosen. The conditional probability distribution for $I_{i, j}$ is calculated based on equation 9. The distributions for $\lambda_{i, j}$ and $M_{U i, j}$ equal the identity matrix, as the parameters are time invariant. $F_{i, j}$ is in the state failure, if $D_{i, j}$ is in the last state corresponding to a damage size larger than 1.

Table 2. Intervals for parameters in the damage model.

\begin{tabular}{|l|l|l|l|}
\hline Variable & Interval size & $\begin{array}{l}\text { Nunber } \\
\text { of states }\end{array}$ & Interval boundaries \\
\hline$D$ & Exponential & 30 & $0,10^{-4}: 1, \infty$ \\
\hline$A$ & Uniform & 10 & $-\infty,-3 \sigma: 3 \sigma, \infty$ \\
\hline$M_{U}$ & Uniform & 10 & $-\infty,-3 \sigma: 3 \sigma, \infty$ \\
\hline$\lambda$ & Uniform & 40 & $0: 1$ \\
\hline$F$ & - & 2 & Failure/no failure \\
\hline$I$ & - & 2 & Detection/no detection \\
\hline
\end{tabular}

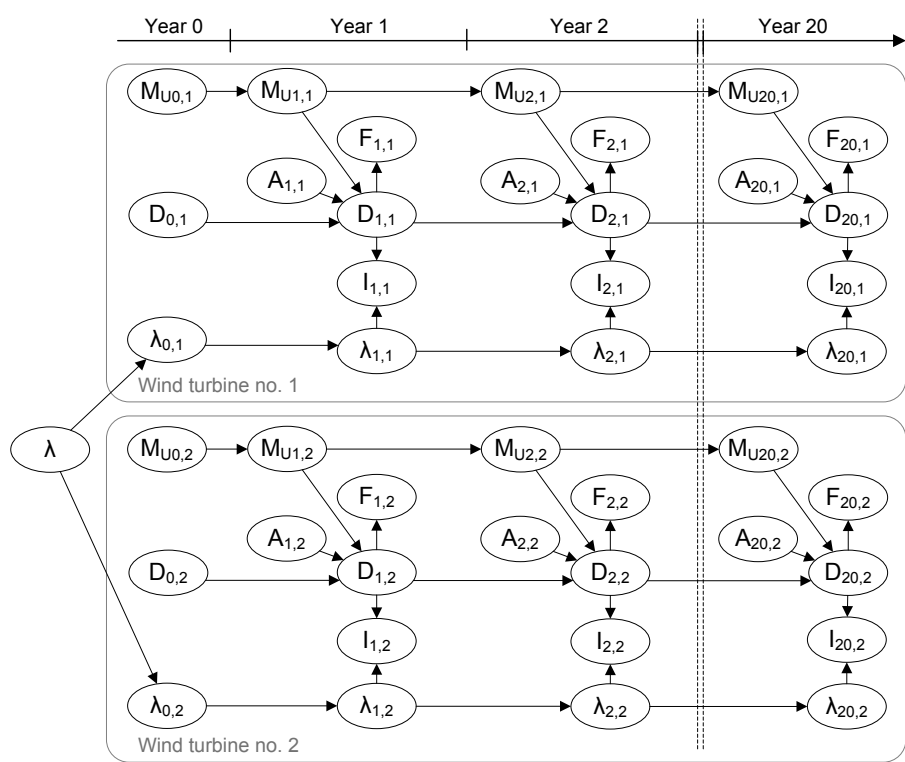

Fig. 6. Bayesian network for learning of the parameter $\lambda$ for two wind turbines and a lifetime of 20 years.

\section{Results}

The model presented can be used to estimate $\lambda$ if data from past cases are available. In this example, the data is generated based on the distributions given in table 1 and equations 7, 8, and 9. For each calculation, the number of wind turbines (number of past cases) and the real value of $\lambda$ are chosen, and data is generated. The evidence for $I_{i, j}$ and $F_{i, j}$ are entered into the network, and the probability distributions are updated.

In Fig. 7 the posterior distributions are shown for $\lambda=0.4$ for different numbers of wind turbines. These distributions depend on the generated data, and will differ if new datasets are generated. However, the Fig. shows that the uncertainty on the estimate decreases with increasing number of wind turbines, and the found distribution predicts a value close to the true value.

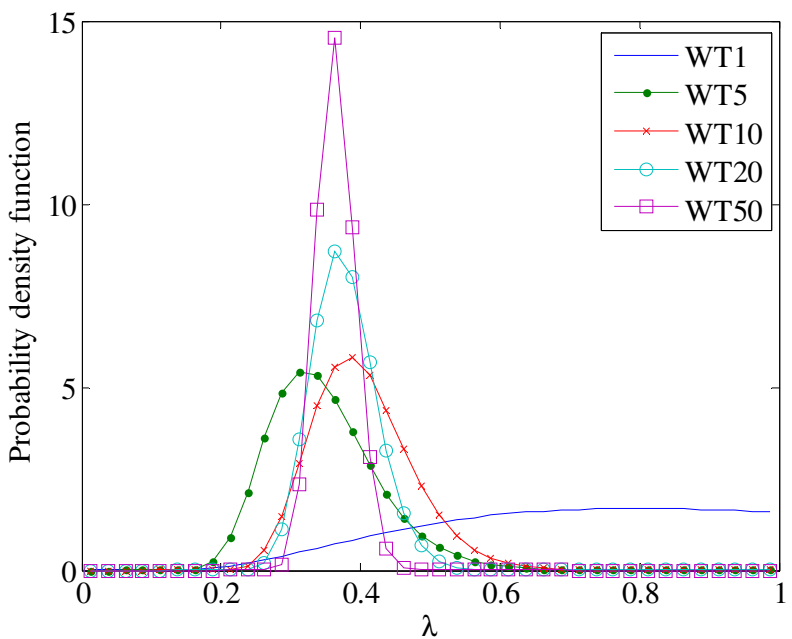

Fig. 7. Probability density function for different numbers of wind turbines for $\lambda=0.4$. 
Fig. 8 shows the posterior distributions for $\lambda$ for the case with 10 wind turbines and a true value of $\lambda$ of $0.2,0.4,0.6$, and 0.8 . For small values of $\lambda$ the standard deviation is smaller, and the estimate is closer to the correct value. This is explained by the shape of the PoD curves, as the curve is steeper for small values of $\lambda$.

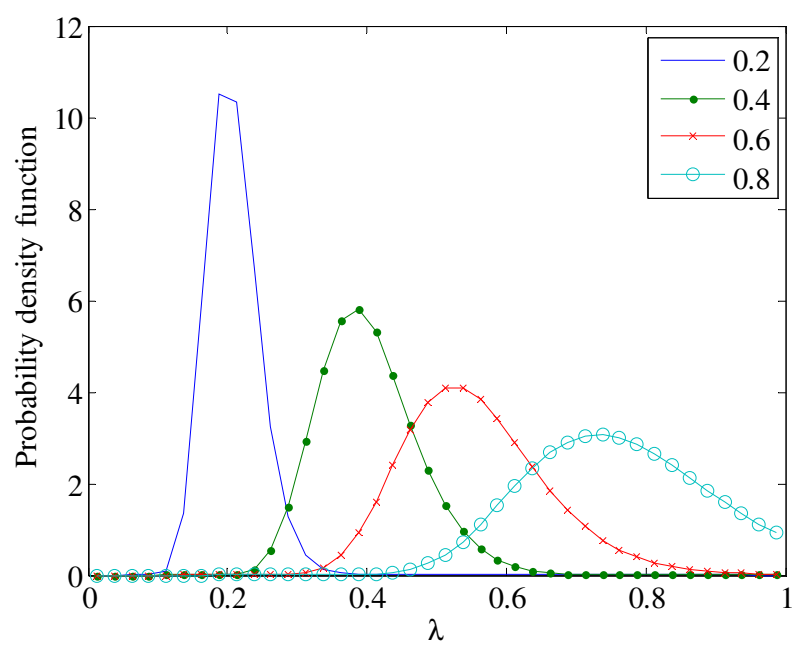

Fig. 8. Probability density functions for different values of $\lambda$ for 10 wind turbines.

\section{DISCUSSION}

The example shows that it is possible to estimate the parameter in an indicator model using past data from the indicator and information about failures only. However, no model uncertainties were assumed to be present, as the data was sampled from the same distributions as the ones being used in the network. Further, the PoD model was assumed to be correct.

For real applications, where a real dataset is used, there would be epistemic uncertainties on the damage and indicator model, and more data would be required to give a good estimate. Furthermore, the used indicator model might not be accurate, and more different model could be tried out. Alternatively, learning could be used to find the conditional distribution for an indicator model not restricted to parameters.

For risk-based planning, the found distribution for $\lambda$ should be used in the networks used for decision making. When new information is entered into the network, the distribution will still be updated sequentially. However, if much past data is available and $\lambda$ can be determined with small uncertainties, it could be modeled as a deterministic parameter to reduce the number of nodes in the Bayesian network and thereby the computational time. See (Nielsen and Sørensen 2010) for examples where Bayesian networks are used for risk-based decision making for maintenance for offshore wind turbines.

\section{CONCLUSIONS}

This paper concerns the development of models for decision problems for risk-based planning. A prior damage model can be combined with data from load measurements, inspections, and the SCADA system to improve the estimate of the probability of failure. Bayesian networks are suitable for Bayesian updating of the probabilities sequentially during the lifetime.

Some data are indicators of the damage size. For instance, alarm thresholds can be set based on one or more SCADA channels, and the occurrence of alarms indicate that damages could be present. Similarly, non-occurrence of alarms indicates that damages are not present. Updating using indicators requires that a model is available for the uncertainty of the data. Past data can be used to establish the indicator model. Either the model can be learnt completely from data using learning of Bayesian networks, or parameters in the indicator model can be learnt using propagation of evidence.

An application example showed that the parameter in a single parameter indicator model could be determined very well based on past data, in the case where the damage model correctly described the deterioration. Future work should aim at using real data measurements for the updating, to assess the additional challenges being present there.

\section{ACKNOWLEDGEMENTS}

The work presented in this paper is part of the project "Reliabilitybased analysis applied for reduction of cost of energy for offshore wind turbines" supported by the Danish Council for Strategic Research, grant no. 2104-08-0014. The financial support is greatly appreciated.

\section{REFERENCES}

Faulstich, S., Hahn, Lyding, P, and Tavner, P (2009). "Reliability of offshore turbines - identifying risks by onshore experience." In European Offshore Wind 2009 Conference proceedings.

Jensen, FV, Nielsen, TD (2007). Bayesian Networks and Decision Graphs, Information Science and Statistics, Springer.

Nielsen, JJ, Sørensen, JD (2010). "Bayesian networks as a decision tool for O\&M of Offshore Wind Turbines" In Proceedings of the 5th International ASRANet Colloquium.

Obdam, T, Rademakers, L, Braam H (2009). "Flight leader concept for wind farm load counting final report." Technical Report ECN-E-09068 .

Spinato, F, Tavner, PJ, van Bussel, GJW, Koutoulakos, E (2009). "Reliability of wind turbine subassemblies." IET Proceedings, Renewable Power Generation 3(4) pp 387-401.

Straub, D (2009), Stochastic modeling of deterioration processes through dynamic Bayesian networks", Journal of Engineering Mechanics, Vol. 135, No 10, pp 1089-1099.

Sørensen, JD (2009). "Framework for risk-based planning of operation and maintenance for offshore wind turbines", Wind Energy Vol 12, No 5, pp 493-506. 



\section{A.5 Paper 5}

Title:

\section{Maintenance Optimization for Offshore Wind Turbines using POMDP}

Authors:

Nielsen, J. S. and J. D. Sørensen.

Conference:

IFIP WG 7.5 Working Conference on Reliability and Optimization of Structural Systems 2012, AUA, Yerevan, Armenia. 



\title{
Maintenance optimization for offshore wind turbines using POMDP
}

\author{
J.S. Nielsen \& J.D. Sørensen \\ Aalborg University, Denmark
}

\begin{abstract}
In this work, a Partially Observable Markov Decision Process (POMDP) is used for decision support for offshore wind turbines. The optimal decision policies for inspection and repair are obtained for each time step dependent on the belief state for the damage state after monitoring, and thus dynamic programming can be used. Seasonal weather variations are included through their influence on weather constrains for inspections and repairs, as well as costs to lost production, when corrective repair is not possible after failure. Application of the model is illustrated through an example, where the main bearing is considered. Optimization is initially performed for one component, and decision making for an entire wind farm is considered by using revised decision policies, when mobilization costs are already paid for another repair. The total costs are calculated for an entire wind farm using simulation and by using the identified decision policies.
\end{abstract}

\section{INTRODUCTION}

Maintenance decision making for offshore wind turbines is both an important and complex topic. Important because maintenance costs are high and need to be reduced to make offshore wind energy more competitive to other energy sources. Complex because decisions need to be made taking into account entire wind farms, costs to inspections, preventive repairs, corrective repairs and lost production, and weather constrains are present for maintenance actions. Furthermore, large uncertainties are present for the deterioration processes and condition monitoring information.

For many mechanical components, more failures can be observed than foreseen in the design, because the actual behavior for e.g. bearings and gears is different compared to the design assumptions. This leads to large costs for corrective and unplanned preventive maintenance. These costs can potentially be reduced if decisions on inspections and preventive maintenance are made with support from risk-based methods.

\section{PRE-POSTERIOR DECISION PROBLEM}

Maintenance planning for offshore wind turbines basically contains decisions regarding inspections and repairs. If the lifetime is divided into a discrete number of time steps and decisions regarding inspections and repairs are made at each time step, the associated simplified decision tree is shown in Figure 1. 


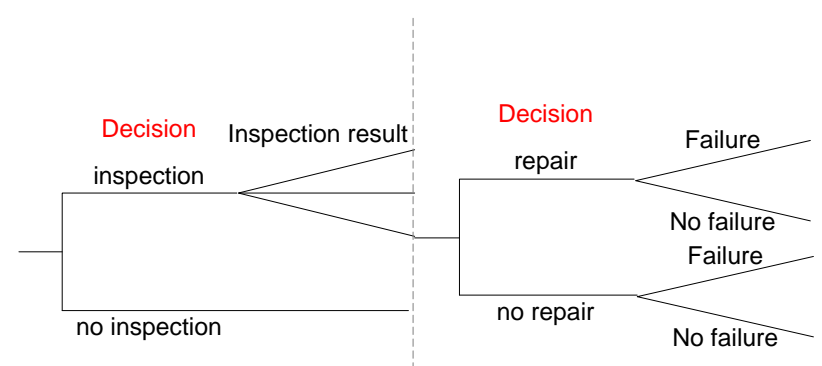

Figure 1: Simple decision tree for inspection and repair planning. Should be repeated for each time step.

At the dashed vertical lines all branches continue as the showed one.

This decision problem contains two decisions at each time step, and earlier decisions influence later decisions; therefore, the problem is a pre-posterior decision problem. For pre-posterior decision problems, optimal decisions should be made such that the expected costs are minimized. When a decision is made, it is necessary to take into account the optimal decisions for all future decisions. The number of branches to evaluate in the decision tree increase exponentially with the number of time steps, and in the unreduced form it becomes intractable. Various approximations can be applied to make it possible to solve the problem. In this paper, the decision process is modeled as a Partially Observable Markov Decision Process (POMDP).

\subsection{Markov decision problems}

A Markov process has the property that the future is independent of the past given the present. The simplest decision problem related to the Markov process is the discrete Markov Decision Process (MPD). For this kind of problems, the state of nature is fully observed at each time step and a decision is made based on the current state of the system. The effects of the actions related to the decisions are uncertain, and costs are associated with the state of nature and decisions. The optimal decision then only depends on the current state of nature. Therefore, an optimal policy can be found for each possible state of nature by simply calculating the expected costs during the lifetime for all possible policies, and choosing those with lowest costs. (Jensen \& Nielsen 2007)

Often, the deterioration state is not fully observed, but instead only observed through some indicator. This is called a Partially Observable Markov Decision Process (POMDP). This means that all past observations are relevant when the decision is made, and not only the present one. However, the past observations can be summarized by using Bayes rule to calculate the probability distribution for the present state of nature (belief state) given the observations. Then, the optimal policy can be found for a given belief state. (Jensen \& Nielsen 2007)

For unbounded time horizons, the strategies are stationary. This means that the optimal policy is the same for all the time steps. For bounded time horizons, the policies will be different at different time steps. Near the end of the lifetime, it will not be cost-effective to make expensive repairs, even though it would be at the same probability belief state at earlier time steps. In this paper, only bounded time horizons are considered.

The advantage of the Markov decision models is that only the current belief state is of importance. This means that Dynamic Programming, introduced by Bellman in 1957 can be used (Dasgupta et al. 2006). When only the current belief state is of importance, the optimal decision can be found for all possible belief states for each time step, by starting from the last time steps, and using the optimal decisions found there, when earlier time steps are evaluated. Then the computational time is only linear with the number of time steps, and not exponential as for a traditional decision tree.

In reality, for discrete state spaces, the belief state is a continuous valued vector with unity magnitude. Therefore, there are infinitely many possible belief states. One way to solve this is to discretize the belief vectors, and interpolate the costs from other belief states between these states (Faddoul et al. 2011).

The classical POMDP has an uncertain inspection at the beginning of each time step, and the decision is made on whether a repair should be made. Corotis et al. (2005) extended the ap- 
proach to include decisions on various types of inspection and maintenance methods for Riskbased inspection and maintenance planning for bridge girders. Faddoul et al. (2011) further extended the procedure to use submodels for more advanced inspection sequences, for example two inspections at each time step.

The general limitation with Markov models is the Markov assumption of independence between past and future given the present. Deterioration processes are generally not Markovian when epistemic time-invariant uncertainties are dominant. However, if time independent variables are included in the model, the future deterioration state is independent of the past given these time dependent variables (Straub 2009). Therefore, the policies can be found for each time step based on current belief state for the deterioration state and current belief state for the timeindependent variable. This increases the number of calculations for each time step, but it is still only linear with the number of time steps.

For the case presented in this paper, a simple three state model for the component health have been applied, and therefore, time-invariant uncertainties are not included in the model. It can be relevant to use such a simple model in cases, where only limited knowledge about the deterioration processes is available. This is the case for many wind turbine components, as the observed behavior is different than the behavior expected from the theoretical models.

\section{MODEL}

The model presented in this section takes into account condition monitoring and inspections and optimal decision policies are found for each time step for the decision on inspections and repairs. The influence of the weather on accessibility has been included, such that it is uncertain if inspections and repairs are possible in a given time step. In each time step, the following takes place:

- Monitoring result is obtained

- Decision on inspection followed by inspection, if possible

- Decision on preventive repair followed by preventive repair, if possible

- Possible failure due to deterioration followed by corrective repair, if possible

The decision tree considered in the model is shown in Figure 2.

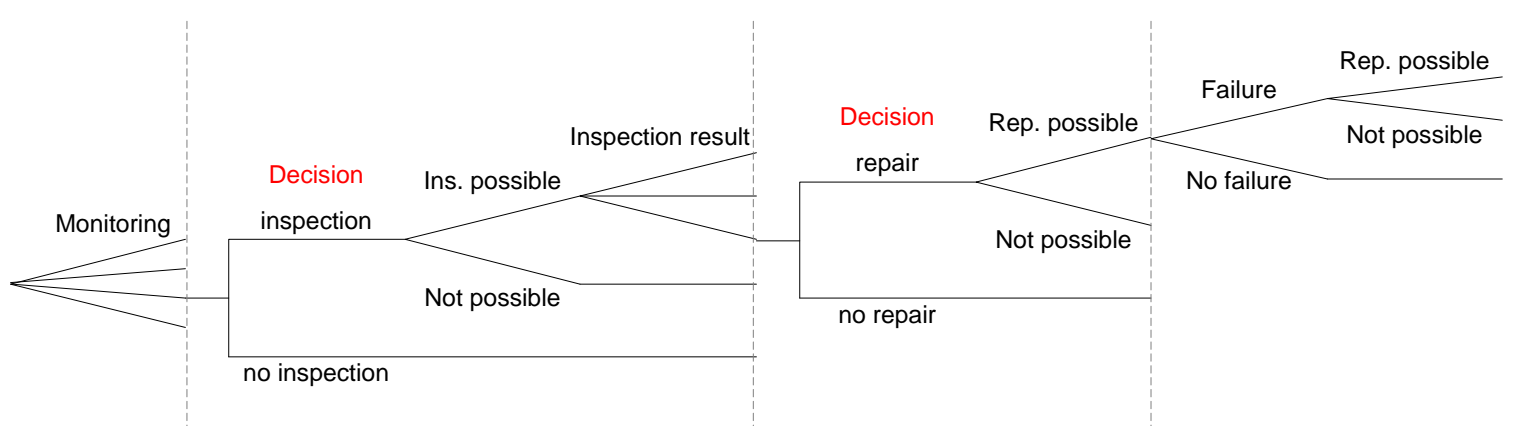

Figure 2: Decision tree. Should be repeated for each time step. At the dashed vertical lines all branches continue as the showed ones.

In this model, the health of the component can take three states; healthy, damaged, and failed. At each time step, there is a probability that the component advances to the following health state, else it remains at the current health state. If a component has failed, it is known, and only corrective repair is possible. Therefore, the belief state for the component health at the time of the decisions can be described by one continuous number, the probability of being damaged, $\mathrm{P}(\mathrm{dmg})$. The model can be extended to more damage states, and in that case the belief state for the component health will be a vector. 
The component is assumed to be healthy at time step 1 , and all repairs are perfect, and will therefore bring the component to the healthy state.

The costs included in the model are cost of inspections, preventive repairs, corrective repairs, and lost production. Lost production is incurred when failure happens, and in the following time steps, if corrective repairs are not possible.

\subsection{Weather constrains}

For offshore operations, vessels have limitations with regard to wave height and wind speed when a wind turbine needs to be accessed, and repair actions have to be made. If the duration of the operation as well as the weather limits are known and long term time series from the location are available, the probability of having an appropriate weather window in a given month of the year can be found. This probability can be found for all months and for all types of operations. Generally, the longer the weather window and the stricter the weather requirements, the larger is the probability that operations are not possible in a given time step. In the model it is assumed that repairs have stricter requirements than inspections, and corrective repairs have stricter requirements than preventive repairs. Therefore, at each time step, there should be correlation between whether inspections are possible and whether repairs are possible, such that repairs are not possible if inspections are not possible. However, if inspections are possible, repairs can still be impossible. The probabilities included in the model are therefore:

- Probability of inspection being possible

- Probability of preventive repairs being possible given that inspections are possible

- Probability of corrective repairs being possible given than preventive repairs are possible

In the model, the year has been divided in seasons, and the probabilities of operations not being possible are constant through each season. Initially these (conditional) probabilities have all been set to 0.1 .

\subsection{Optimization procedure}

The principle behind the optimization calculations is dynamic programming, as explained in section 2.1. First, the state space for the component health is discretized into a finite number of belief states. For all these belief states, the optimal decision policies are found sequentially for each time step, starting at the last time step. At the final branches, at each time step, the expected costs for the remaining life time are needed in the calculations. These costs only depend on the belief state, and can be found by interpolation between the values found at the following time step. Further, each time step was divided in two, such that the interpolation was performed after the monitoring branches and after corrective repair branches. This reduces the number of branches to calculate at each time step by a factor three. At each time step, the probabilities and expected costs for all branches are calculated. This includes using Bayes rule for updating of the health state after monitoring and after inspections. The decision policies are found such that the expected costs are minimized.

\section{EXAMPLE}

The purpose of this example is to test that the model works as intended. The model could be used for various components, and in this example the main bearing is considered. The time step length is chosen to one month, and the lifetime of the turbine is set to 20 years.

\subsection{Deterioration model}

The deterioration model has been set such that the mean time to failure is 40 years, and the time in the damaged state is shorter than time in the healthy state. The transition probabilities are: 


$$
P\left(D_{i} \mid D_{i-1}\right)=\left[\begin{array}{ccc}
0.9972 & 0 & 0 \\
0.0028 & 0.9917 & 0 \\
0 & 0.0083 & 1
\end{array}\right]
$$

where there is a column for each old damage state. The belief state for the damage state is updated by multiplying this matrix by the old damage state, represented by a column vector:

$$
P\left(D_{i}\right)=P\left(D_{i} \mid D_{i-1}\right) P\left(D_{i-1}\right)
$$

The optimal policies have been found for belief states in 0.01 discretization.

\subsection{Monitoring and inspection model}

A condition monitoring system based on vibration measurements is assumed to be available, and at each time step it is evaluated whether the measurements are low, medium, or high, or whether failure has occurred. This gives four outcomes for the monitoring result. The conditional probabilities for these outcomes as set to:

$$
P\left(\text { Mon }_{i} \mid D_{i}\right)=\left[\begin{array}{ccc}
0.6 & 0.1 & 0 \\
0.3 & 0.3 & 0 \\
0.1 & 0.6 & 0 \\
0 & 0 & 1
\end{array}\right]
$$

It is then decided, whether an inspection should be made, and if made, the inspection results in detection of damage or no detection of damage. This gives three outcomes for the inspection result, where the third one is failure:

$$
P\left(\text { Ins }_{i} \mid D_{i}\right)=\left[\begin{array}{ccc}
0.9 & 0.2 & 0 \\
0.1 & 0.8 & 0 \\
0 & 0 & 1
\end{array}\right]
$$

Based on these probabilities, the probability for each inspection outcome can be found, and the belief state for the damage given each observation can be found using Bayes rule.

\subsection{Approximation for wind farm}

The optimal policies are found using the POMDP for a single turbine. When decisions are made for an entire wind farm, system effects can be considered. Here it is assumed that the mobilization cost for the repair vessel is a significant contributor to the total cost of a preventive repair, and if several repairs are made at the same time step, the mobilization costs only need to be paid once. To include this, the optimal repair policy has been found during the optimization procedure for the case, where the mobilization costs are zero at the current time step, due to another repair paying them, but everything in the future is as in the normal case. These revised policies are more cost-efficient than the policies found without taking system effects into account, but the solution is still suboptimal.

\subsection{Costs}

The costs are in the example set to the following relative values:

- Inspection: 1

- Preventive repair

- Mobilization costs: 100

- Unit repair cost: 100

- Corrective repair 
- Lost production per month: 100

- Repair costs: 500

For simplicity, discounting has not been included, but this could be implemented.

\subsection{Policies}

The primary outcomes of the decision analysis are the decision policies. In Figure 3, the optimal decision policies are shown for inspections and preventive repairs. Furthermore, the revised policies in case of repairs of other turbines at the same time step are also shown. All decisions are shown as function of time step and the probability of a damage being present, updated using the monitoring outcome. For preventive repairs, the decision further depends on whether an inspection has been made, and the result of the inspection.

Generally, no inspections or preventive repairs should be made near the end of the life time. The scatter (the white lines) for the inspection policies in Figure 3 is due to the expected costs for the two decisions being very close. Therefore, it is almost indifferent whether inspections are made.
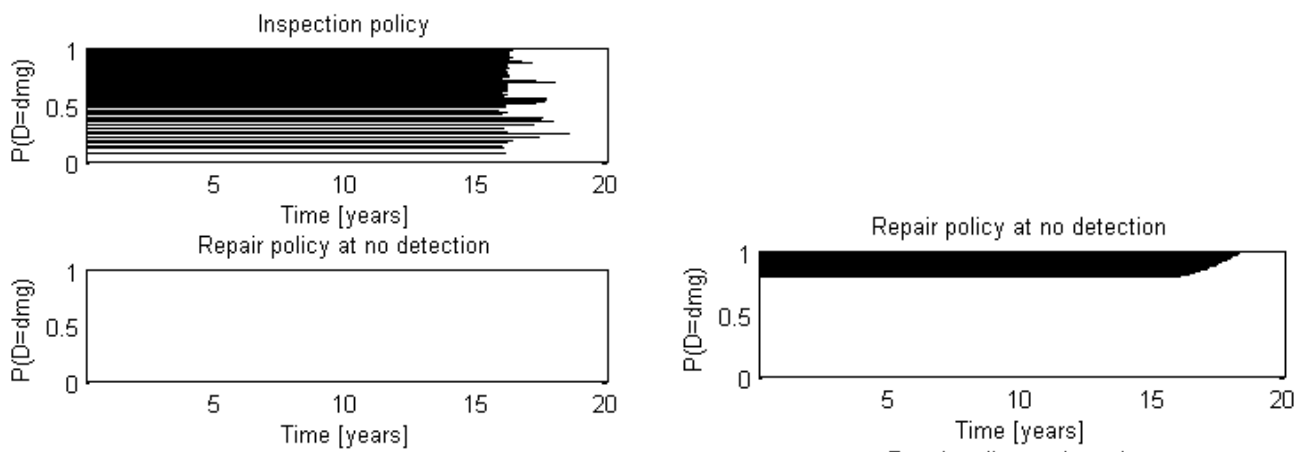

Repair policy at detection

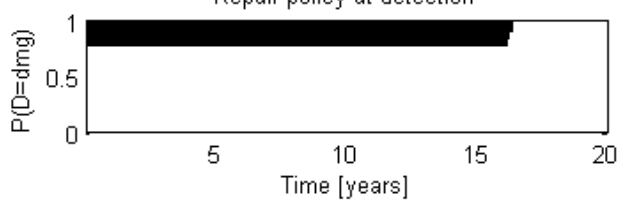

Repair policy at detection
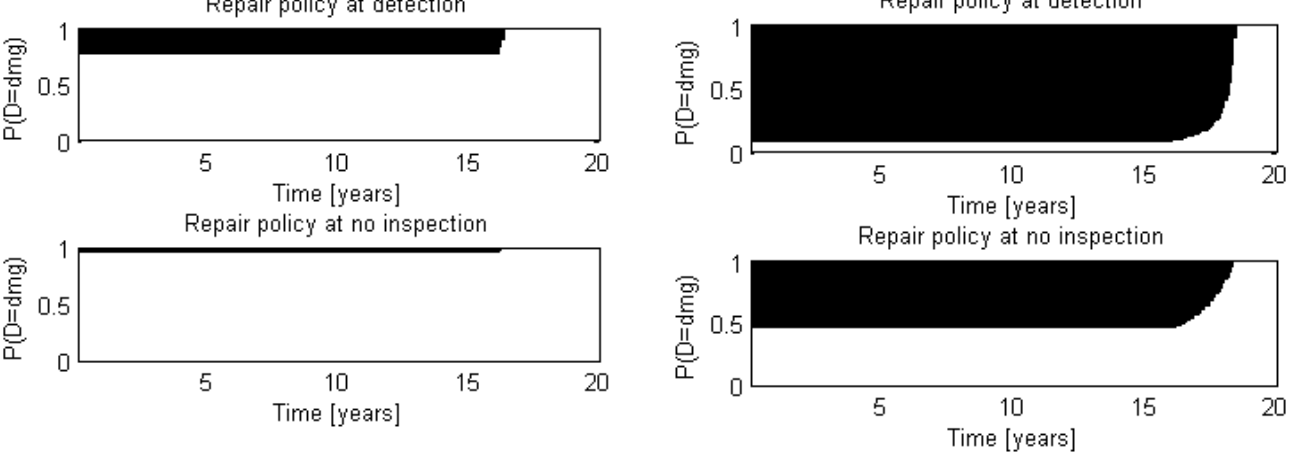

Figure 3: Policies for a wind turbine with (left) and without (right) mobilization costs. At a given time step, if the probability of a damage being present is within the black areas, inspections/repairs should be made.

\subsection{Simulation for a wind farm}

Simulations have been performed through the lifetime for a wind farm with 40 wind turbines. At each time step, the belief state has been updated for each wind turbine, and the optimal decision is chosen from the found decision policies. The revised decision policies with no mobilization costs are used for the remaining turbines, if any of the turbines are already being preventively or correctively repaired.

Figure 4 shows the main events of such a simulation. When repairs, but not repair decisions, are marked, the revised decision policies are the reason for the repairs. 


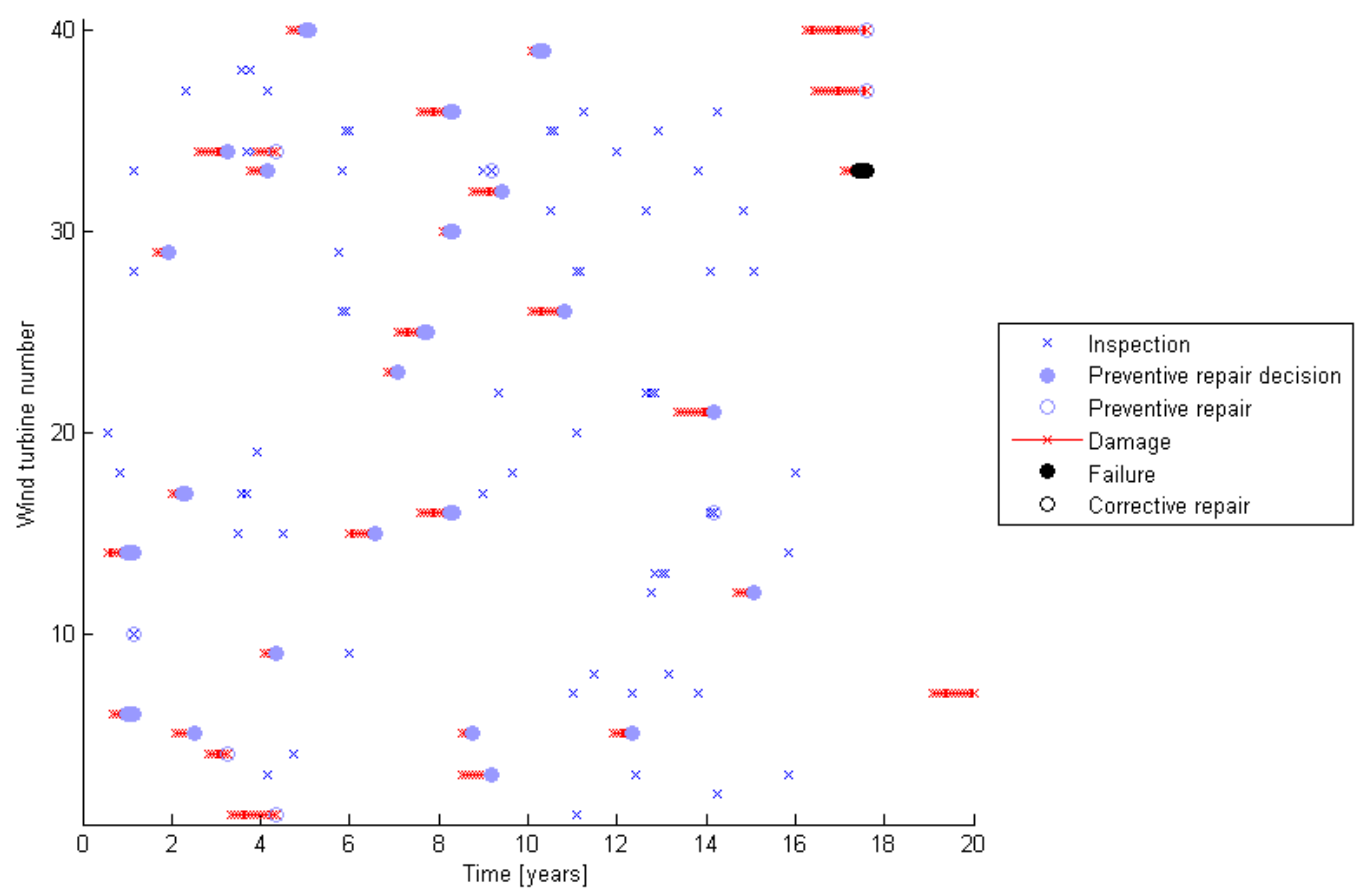

Figure 4: Simulation for a wind farm with revised policies found using POMDP.

The total expected lifetime costs have been found using only the policies with mobilizations costs included to approx 5300, and using also the revised policies to approx 5000. The costs are shown in Figure 5 divided into categories, and the 95\% confidence intervals for the mean of the total costs are also shown. The total costs are 5\% smaller when the revised policies are also used. Here, the mobilization costs and failure costs are smaller, while the unit repair costs are larger due to more preventive repairs.

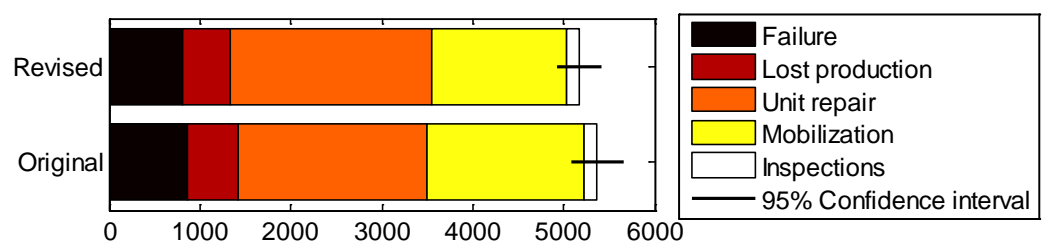

Figure 5: Comparison of costs for original policies and improved polices.

\subsection{Simulation for a wind farm with harsh winter}

For the simulations performed above, all conditional probabilities for repairs not beings possible were 0.1 for all months. To evaluate the influence of a harsh winter, all the conditional probabilities are set to 0.9 for the first and fourth quarter of the year. The decision policies for this case is shown in Figure 6.

Here, the policies are periodic and depend on the month of year. Two months before the winter starts, repairs should be made for smaller probabilities of damage than in the beginning of the mild season. During the first part of the winter, even more repairs should be made, even though it is rarely possible. The total costs are here approx 5400 and 5200, when the original and revised policies respectively are used, which is more than the case with constant mild weather. 

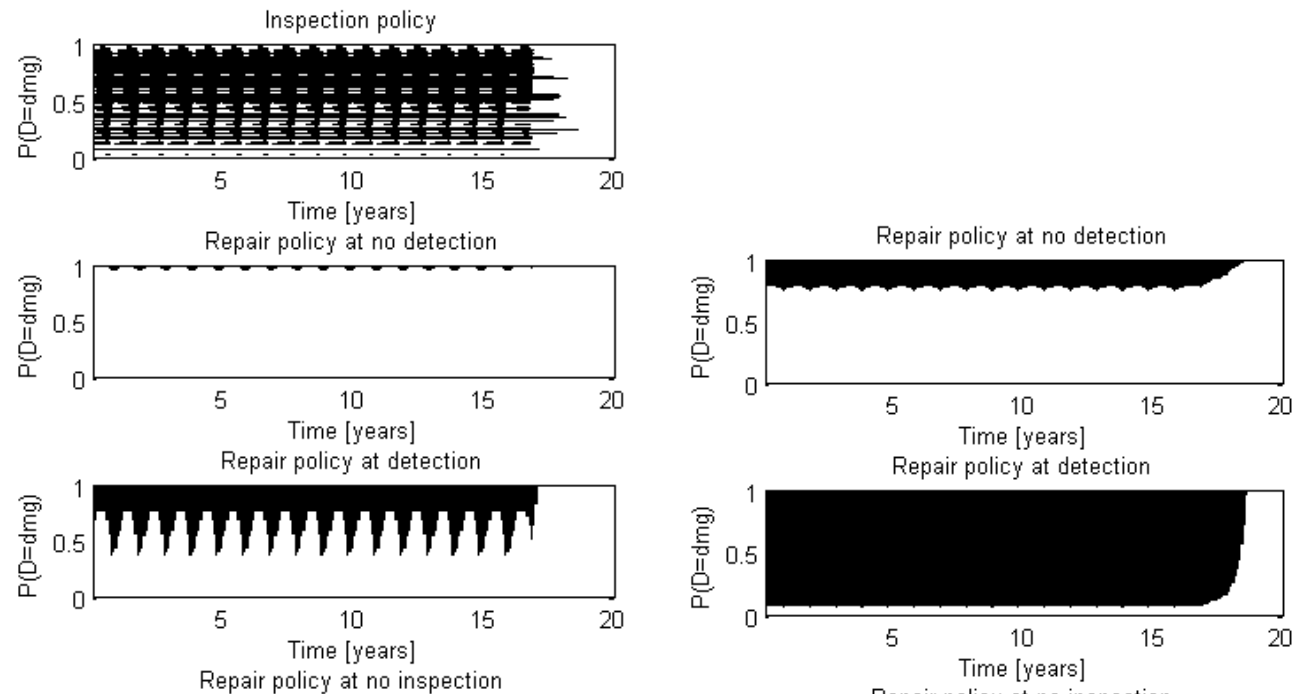

Repair policy at no inspection
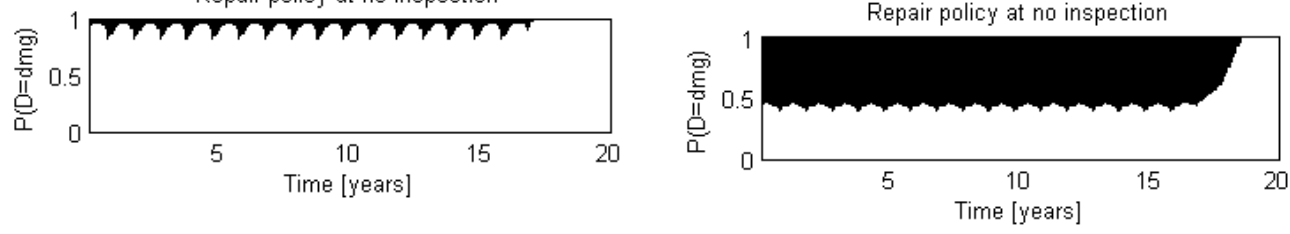

Figure 6: Policies for a wind turbine when the autumn and winter is harsh. In the black areas, inspections/repairs should be made.

\section{CONCLUSIONS}

A model based on POMDP was presented for decision support for inspections and repairs for offshore wind turbine components. When the weather was constant during the year, the decision policies were constant until the last few years of the lifetime, and in the case with seasonal changes in weather, the policies were periodic. Approximate decision making for a wind farm was performed by using revised decision policies for the remaining turbines, when other repairs were already being performed. This gave a small reduction in the expected costs.

\section{ACKNOWLEDGEMENTS}

The work presented in this paper is part of the project "Reliability-based analysis applied for reduction of cost of energy for offshore wind turbines" supported by the Danish Council for Strategic Research, grant no. 2104-08-0014. The financial support is greatly appreciated.

\section{REFERENCES}

Corotis, R.B., Ellis, J.H. \& Jiang, M., 2005. Modeling of risk-based inspection, maintenance and-life cycle cost with partially observable Markov decision processes. Structure and Infrastructure Engineering, 1(1): 75-84.

Dasgupta, S., Papadimitriou, C.H. \& Vazirani, U.V., 2006. Algorithms, McGraw-Hill.

Faddoul, R., Raphael, W. \& Chateauneuf, A., 2011. A generalised partially observable Markov decision process updated by decision trees for maintenance optimisation. Structure and Infrastructure Engineering, 7(10): 783-796.

Jensen, F.V. \& Nielsen, T.D., 2007. Bayesian Networks and Decision Graphs, New York, Springer.

Straub, D., 2009. Stochastic Modeling of Deterioration Processes through Dynamic Bayesian Networks. Journal of Engineering Mechanics, 135(10): 1089-1099. 


\section{A.6 Paper 6}

\section{Title:}

\section{Analysis of pitch system data for condition monitoring}

Authors:

Nielsen, J. S., R. P. v.d. Pieterman and J. D. Sørensen.

Published in:

Wind Energy (Published online), 2013. DOI: 10.1002/we.1586. 



\title{
RESEARCH ARTICLE
}

\section{Analysis of pitch system data for condition monitoring}

\author{
Jannie S. Nielsen ${ }^{1}$, René P. van de Pieterman ${ }^{2}$ and John D. Sørensen ${ }^{1}$ \\ 1 Aalborg University, Sohngårdsholmsvej 57, 9000 Aalborg, Denmark \\ 2 Unit Wind Energy, Energy research Center of the Netherlands, Petten, The Netherlands
}

\begin{abstract}
Measurements of pitch motor torque and current give indirect information about the condition of the pitch system and can therefore potentially be used for condition-based maintenance. This paper presents an analysis of these measurements for a wind turbine, and the measurements are compared with a theoretical model based on aeroelastic simulations. The blade moment is found to have only minor influence on the friction in the blade bearing. The main factors affecting the static friction are the temperature and time after the latest pitch movement. Pitch motor current and torque are proportional at a constant pitch velocity, but the 10 min maximum values are only approximately proportional, because the maximum values occur during acceleration and not simultaneously. These findings are important to consider, if using the pitch motor current or torque as an indicator for the pitch system health is considered. Copyright @ 2013 John Wiley \& Sons, Ltd.
\end{abstract}

\section{KEYWORDS}

pitch system; indicators; operation and maintenance; condition monitoring; wind turbines

\section{Correspondence}

J. S. Nielsen, Department of Civil Engineering, Aalborg University, Sohngårdsholmsvej 57, 9000 Aalborg, Denmark.

E-mail: jjn@civil.aau.dk

Received 6 August 2012; Revised 1 October 2012; Accepted 25 November 2012

\section{INTRODUCTION}

For wind turbines, component failures lead to large costs of operation and maintenance, and also, in the pitch, system failures occur. ${ }^{1}$ Costs of corrective maintenance can be reduced by using preventive maintenance, but the uncertainties on damage models are generally so large that scheduled preventive maintenance may not lead to reduced costs. Instead, condition-based maintenance can be used, where maintenance decisions are made on the basis of the actual health of the components. Information about the component health can be obtained from inspections, from the supervisory control and data acquisition (SCADA) system or from a dedicated condition-monitoring system. Cost optimal maintenance can then be based on risk-based methods, ${ }^{2}$ and here, it is important to consider the uncertainties for the condition-monitoring system. Often, statistical pattern recognition tools are applied to vibration measurements for detection of faults. ${ }^{3,4}$

For electrical pitch systems, the pitch motor (PM) current can potentially be used as an indicator of the health of the system. This is because the current depends on the torque demanded to rotate the blades, and the torque demand will be affected by increased resistance and friction in the system. Furthermore, wear in the gears can lead to dynamic loads, which can also affect the torque. Presently, no condition- monitoring systems using the PM torque or current as indicator have been developed, but it is in the interest of both manufacturers and owners, as such a system can increase the reliability of the pitch system.

In this work, PM torque and current measurements for a wind turbine have been analyzed to determine whether the PM current can potentially be used as an indicator for the health of the system. Part of the work was presented by Nielsen and Sørensen, ${ }^{5}$ who investigated whether the PM current could be used as an indicator of wear in the transmission gear in the pitch bearing. A Bayesian statistical model was used to analyze the development of the maximum PM current reached when the turbine status changed from running to idling. Data from a period before and after preventive maintenance were used in the analysis, but the PM current was not found to be a good indicator for this type of damage because the bearing friction seemed to influence the PM current the most. Factors influencing the bearing friction was taken into account in the analysis, but the changes due to maintenance were still not convincing. 
If a damaged bearing has larger friction than a healthy one, and larger friction leads to larger PM torque, it might be possible to use the PM torque or current as an indicator of the health of the pitch bearing. Recent research show that a linearly increasing friction coefficient could be detected, given that reliable measurements of PM torque and blade moments are available, as well as a reliable model for the PM torque including a bearing friction model. ${ }^{6}$

The PM torque is the most direct measure of the bearing friction, but measurement systems for PM torque and blade moments are expensive and need to be maintained, and are therefore not standard measurements for wind turbines. The PM current is known by the control system (PLC), and for some turbines, it is included in the SCADA data. Therefore, it would be preferable if the PM current could be used as an indicator for the bearing health instead. The PM power has also been considered as an indicator but was unfortunately not available as a parameter in the SCADA system.

The PM torque is affected by various factors, and the bearing friction itself can also be affected by other factors than the health state. Thus, it is crucial to understand what influences the PM torque and current measurements, and to include this knowledge in the fault detection algorithms in order to increase the reliability of the condition-monitoring system. Therefore, measurements in this paper are compared with a theoretical model for the PM current under normal/healthy conditions. The paper investigates which factors influence the PM torque including the running friction by using a Bayesian statistical analysis. Furthermore, factors influencing the maximum values including the static friction are investigated. A condition-monitoring system based on the maximum values of the PM current would be simplest, and therefore, the relationship between maximum PM current and torque is also analyzed.

\section{SYSTEM DESCRIPTION}

The turbine considered in this analysis is a multi-megawatt pitch-controlled wind turbine with an electrical pitch system. The purpose of the pitch system is to control the power output and loads on the wind turbine, by turning the blades around their own longitudinal axes and thereby change the aerodynamic forces. During normal production below rated wind speed, the blades are fixed in position at $0^{\circ}$ to maximize the power output. During production above rated wind speed, the blades are pitched with the leading edge towards the wind so as to reduce the loads while continuing to produce rated power. If the wind speed reaches the cut-out wind speed, the turbine is stopped by turning the blades to $90^{\circ}$. For wind speeds below cut-in, the turbine is set in an idling position with the blades at an angle of $70^{\circ}$ where the rotor will rotate slowly.

For the pitch system, the following operational states of the turbine to which the measured data is classified will be considered:

All:

Starting:

Running, no pitching: The turbine is producing power with the pitch angle $0^{\circ}$.

Running to idling:

Running, pitching:

Braking:

The turbine status changes from running to idling. The pitch angle is adjusted from 0 to $70^{\circ}$.

The blades are pitched to an angle different than $0^{\circ}$ during production. This normally happens at wind speeds above $15 \mathrm{~m} \mathrm{~s}^{-1}$, where the blades are pitched to reduce the loads, but it can also happen below rated wind speed due to e.g. sound reduction.

The turbine status changes from running to stopped. The pitch angle is adjusted from 0 to $90^{\circ}$.

Generally, the system consists of the following components: pitch control, motor, brake, gearbox, pinion and slewing bearing with an internal gear, as shown in Figure 1. Furthermore, the figure shows the location of the torque transducer used for measuring the torque, which is normally not a part of the pitch system.
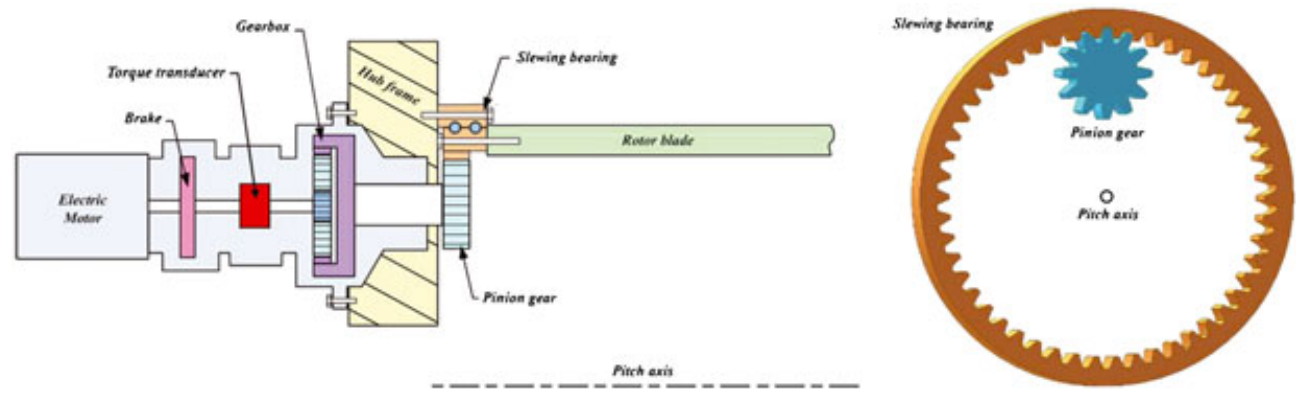

Figure 1. Overview of pitch system, including side view of pitch gear (left) ${ }^{7}$ and front view (sketch) of pitch gear (right). 
The controller determines the amount of current to be delivered to the motor so that the blade angle reaches the set point. The motor is an asynchronous AC motor, where the rotational speed depends on the torque and applied current. At a constant speed, the PM current and torque are proportional to each other.

The pitch gearbox decreases the speed and thereby increases the torque to be delivered to the pinion gear. The pinion gear drives the internal gear in the slewing bearing, which is a double-row ball bearing. The gearbox is lubricated with oil, whereas the bearing and transmission between pinion and blade are lubricated with grease.

\subsection{Loads on the rotor}

The loads on the rotor are transferred to the hub through the pitch system. The rotor is exposed to the following forces:

- Gravitational forces

- Aerodynamic forces (wind loads)

- Centrifugal forces (from the rotation)

- Gyroscopic forces (when yawing)

The gravitational forces are cyclic loads on the blades due to the rotation. The wind loads result in aerodynamic liftand-drag forces, and an aerodynamic moment on the blades. The centrifugal load gives an axial load directed away from the rotor due to the rotation. The forces result in cyclic varying radial forces, axial forces, bending moments and torque.

\subsection{Loads on the pitch system}

The axial and radial forces and bending moments on the blade are transferred to the hub through the slewing bearing. The torque from the external forces attempts to rotate the blades, and the slewing bearing only provides a low friction to counteract the torque. In some cases, the friction is enough to prevent rotation, whereas in other cases the PM needs to counteract the torsion from the blade. The friction in the bearing is expected to depend on the forces and moments on the bearing.

When the blade is being pitched, the PM needs to overcome the torsion in the blades and the inertia and friction forces in the system. The inertia forces arise when the masses in the system accelerate: the gears, bearing and blade. Friction forces are primarily present not only in the slewing bearing but also in the transmission between pinion and blade and in the gearbox.

\subsubsection{Theoretical model.}

The PM has to overcome the blade torsion from external forces, the friction and the inertia forces. The PM torque can be calculated from ${ }^{7}$

$$
T_{\mathrm{m}} \cdot i_{\mathrm{gbx}} \cdot i_{\text {gear }}=T_{\mathrm{b}}+\frac{\dot{\theta}}{|\dot{\theta}|} T_{\mathrm{f}}+\ddot{\theta}\left(\left(i_{\mathrm{gbx}} \cdot i_{\text {gear }}\right)^{2} \cdot I_{\mathrm{gbx}}+I_{\mathrm{b}}\right)
$$

with

$T_{\mathrm{m}}$ PM torque demand

$T_{\mathrm{b}}$ Blade torque from aeroelastic model (includes blade loads and inertia torque from deflection dependant blade inertia $I_{\mathrm{bl}}$ )

$T_{\mathrm{f}}$ Friction torque

$\dot{\theta}, \ddot{\theta}$ Pitch velocity and acceleration

$i_{\text {gbx }}, i_{\text {gear }}$ Transmission ratio of gearbox and blade bearing gear

$I_{\mathrm{gbx}}, I_{\mathrm{b}}$ Inertia of gearbox and blade bearing

According to Harris et al., ${ }^{8}$ a conservative estimate of the running friction torque can be obtained for a single-row ball bearing as

$$
T_{\mathrm{f}}=\mu \frac{d_{\mathrm{m}}}{2}\left(\frac{4.4 M}{d_{\mathrm{m}}}+2.2 F_{\mathrm{r}}+F_{\mathrm{a}}\right)
$$

with

$\mu$ Friction coefficient

$d_{\mathrm{m}}$ Diameter of bearing raceway

$M$ Resultant blade root bending moment

$F_{\text {r }}$ Radial blade force

$F_{\text {a }}$ Axial blade force 
where the moment and forces act on the bearing. The bearing coefficient of friction, $\mu$, is given as $0.003-0.004$. Further, the starting friction can exceed the running friction by a factor 1.5.

According to Rothe Erde Slewing Bearings, ${ }^{9}$ the running friction for ball bearings can be estimated with approximately $\pm 25 \%$ accuracy using the following formula:

$$
T_{\mathrm{f}}=\mu \frac{d_{\mathrm{m}}}{2}\left(\frac{4.4 M}{d_{\mathrm{m}}}+2.2 F_{\mathrm{r}} 1.73+F_{\mathrm{a}}\right)
$$

with $\mu=0.004$ for double-row ball bearings. The only difference between formulae 2 and 3 is the factor 1.73 on the radial force term, but the background for this factor is not given. ${ }^{9}$

In order to calculate the PM torque using equation 1, the blade forces, moments and torque as well as the pitching velocity and acceleration need to be calculated. The calculations used in this analysis was performed by The Energy Research Center of the Netherlands $(E C N)$ in the aeroelastic simulation program PHATAS.

\subsection{Data}

The data used for this analysis comes from a commercially available, multi-megawatt wind turbine on which $E C N$ has performed measurements. A Meteo mast is located close to the turbine and measures, e.g., wind speed, velocity and air temperature at hub height. Measurements similar to those from the PROTEST project ${ }^{7,10,11}$ were available for the turbine for the period October 2009 to April 2011. The measurement system was not maintained after August 2010.

The measurement campaign includes the following measurements, where some of them originate from the PLC system:

- Operational mode (PLC)

- Wind speed (PLC)

- Azimuth angle (blade position in rotor plane) (PLC)

- Pitch angle (PLC)

- Pitch motor active current (PLC)

- Temperatures

- Blade torque

- Blade bending moments (edgewise and flapwise)

- Pitch motor torque

For this analysis, 10 min statistics (mean, standard deviation, minimum and maximum) were available throughout the period, except for periods with errors in the data. Furthermore, time series (128 Hz/32 Hz for PLC) were available in the database.

The blade bending moments were measured using bridges of T-shaped strain gauges and calibrated using the weight of the blade. The blade torsion was measured using 45-45 strain gauges, but the signal is highly influenced by the blade moments and forces, and only an approximate calibration can be performed, as described by Savenij et al. ${ }^{10}$ Comparison of the measured blade torque with the modeled blade torque and measured PM torque indicated that the measured blade torque could not be used for a quantitative analysis. The PM torque was measured using a torque transducer mounted on the low speed shaft between the PM and gearbox, as shown in Figure 1. Detailed descriptions of these types of measurement devices can be found in the PROTEST report. ${ }^{10}$

For this turbine, deterioration of the pitch gears were observed. Preventive repair was performed in May 2010 by dismantling the blades and rotating the slewing bearing with the internal gear by $90^{\circ}$. As described by Nielsen and Sørensen, ${ }^{5}$ this did not significantly influence the peak values of the PM current.

On the basis of the time series of the pitch angle, the pitching velocity and acceleration have been computed. Direct numerical differentiation was not possible because of high-frequency noise; therefore, a Savitzky-Golay filter was applied. This adds some uncertainty to the acceleration in particular.

All values have been normalized because of requirements of anonymity, and the time series have been aligned as well as possible because there were minor delays in the measurement system.

\section{ANALYSIS OF MEASUREMENTS}

Potentially, the PM current and torque can be used as indicators of the health of the pitch system. Often, an indicator will be some value, e.g., a mean or maximum value, which increases with the size of the damage. If unknown factors influence the indicators, it can lead to flawed conclusions. Therefore, it is important to understand the measurements and variations in the measurements. The theoretical model in equation 1 gives an estimate of the PM torque for a healthy pitch system 
under idealized conditions. In this section, the measurements of the PM torque will be compared with the theoretical model to see if there are any variations.

First, an overview of the data is shown in Figure 2, where the 10 min minimum and maximum values of the PM torque are showed for the various operational modes for the measurement campaign before maintenance. The figure clearly shows that the maximum value depends on the operational state and, for some operational states, the wind speed. This is the case for normal production with no pitching.
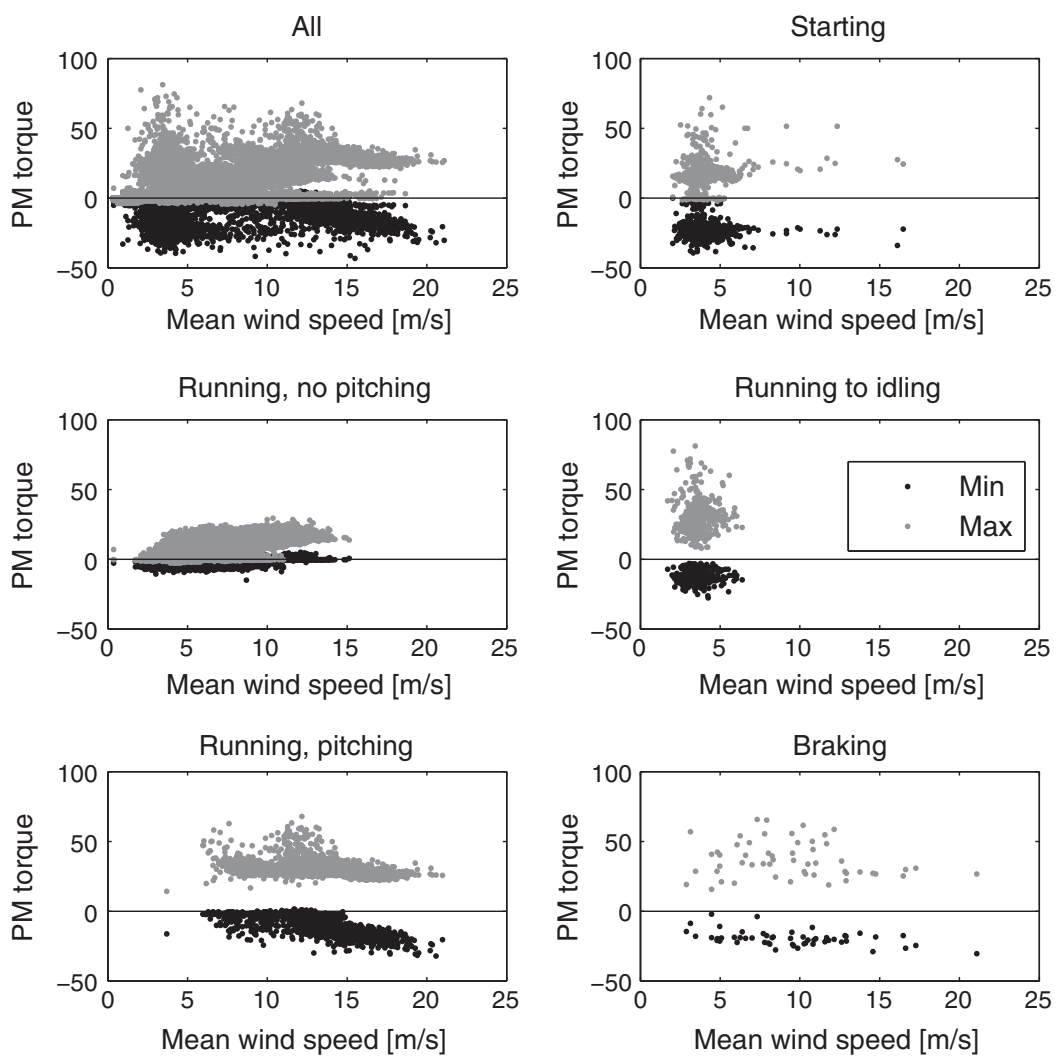

Figure 2. Ten minute maximum and minimum of the PM torque for various operational modes.
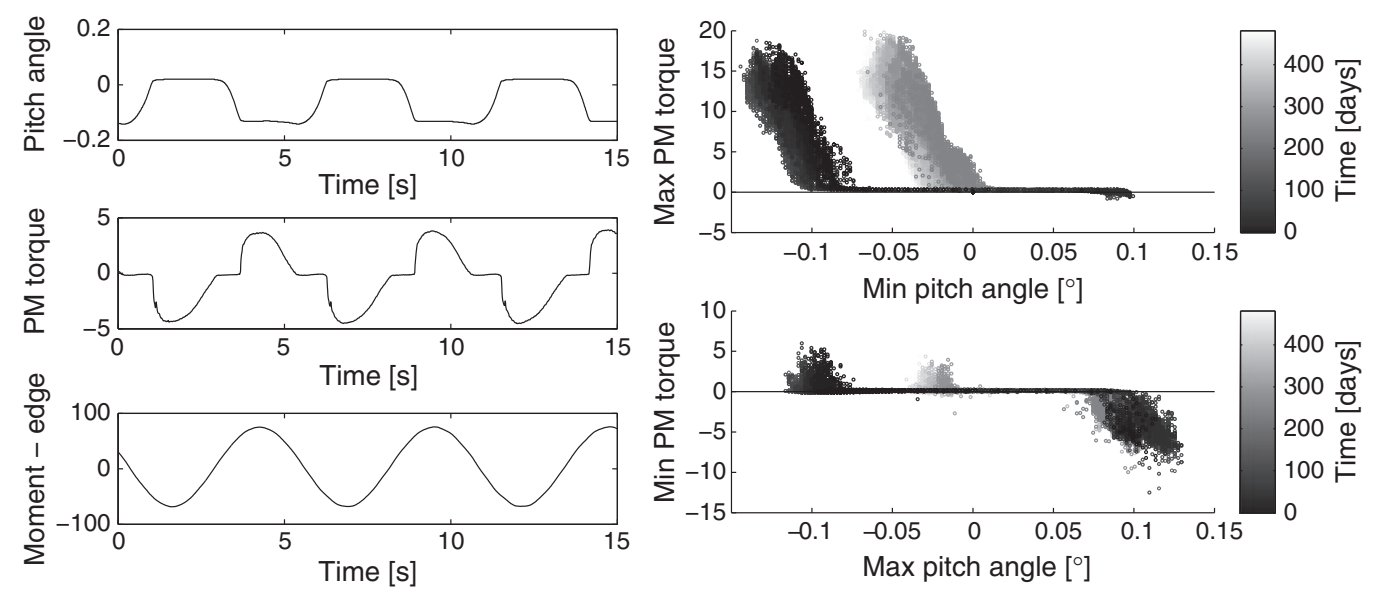

Figure 3. Example of movements during normal production with no pitching (left) and min/max PM torque as function of max/min pitch angle (right). 
When the turbine is producing power below rated wind speed, the role of the pitch system is to keep the blades fixed at the same position, while the external forces produce oscillating torque on the blades. If the blade torque is less than the bearing friction, there is no movement. If the blade torque exceeds the friction, the PM needs to counteract the torque. The PM torque depends on the wind speed for normal production with no pitching, as seen in Figure 2, but the variation is large. In Figure 3 (left), a time series is shown for normal production with no pitching. The pitch angle moves between the outer positions while the PM torque is zero, indicating that the gears in the drive train are not in perfect mesh. Figure 3 (right) shows scatter plots of maximum PM torque and minimum pitch angle as well as minimum PM torque and maximum pitch angle, as these pairs of values occur at the same time. This shows that, in some time series, there are only very small movements, and the measured maximum PM torque is zero. Time is illustrated by color change; dark colors occur before maintenance and lighter colors after maintenance. The figure shows a range with an approximate linear relationship between pitch angle and PM torque, corresponding to an elastic drive train and a range with zero PM torque, indicating free play in the gears. The free play seems to be increasing with time and to be reduced by the maintenance.

The largest values of the PM torque are obtained when the turbine status changes from running to idling, as shown in Figure 2. Here, the blades are initially located at $0^{\circ}$ and are turned to $70^{\circ}$. Large values of the PM torque are also obtained when starting, braking and running above rated, and there is no immediate reason why the largest values are obtained when changing status from running to idling. However, this operational mode could be interesting to consider for condition-based
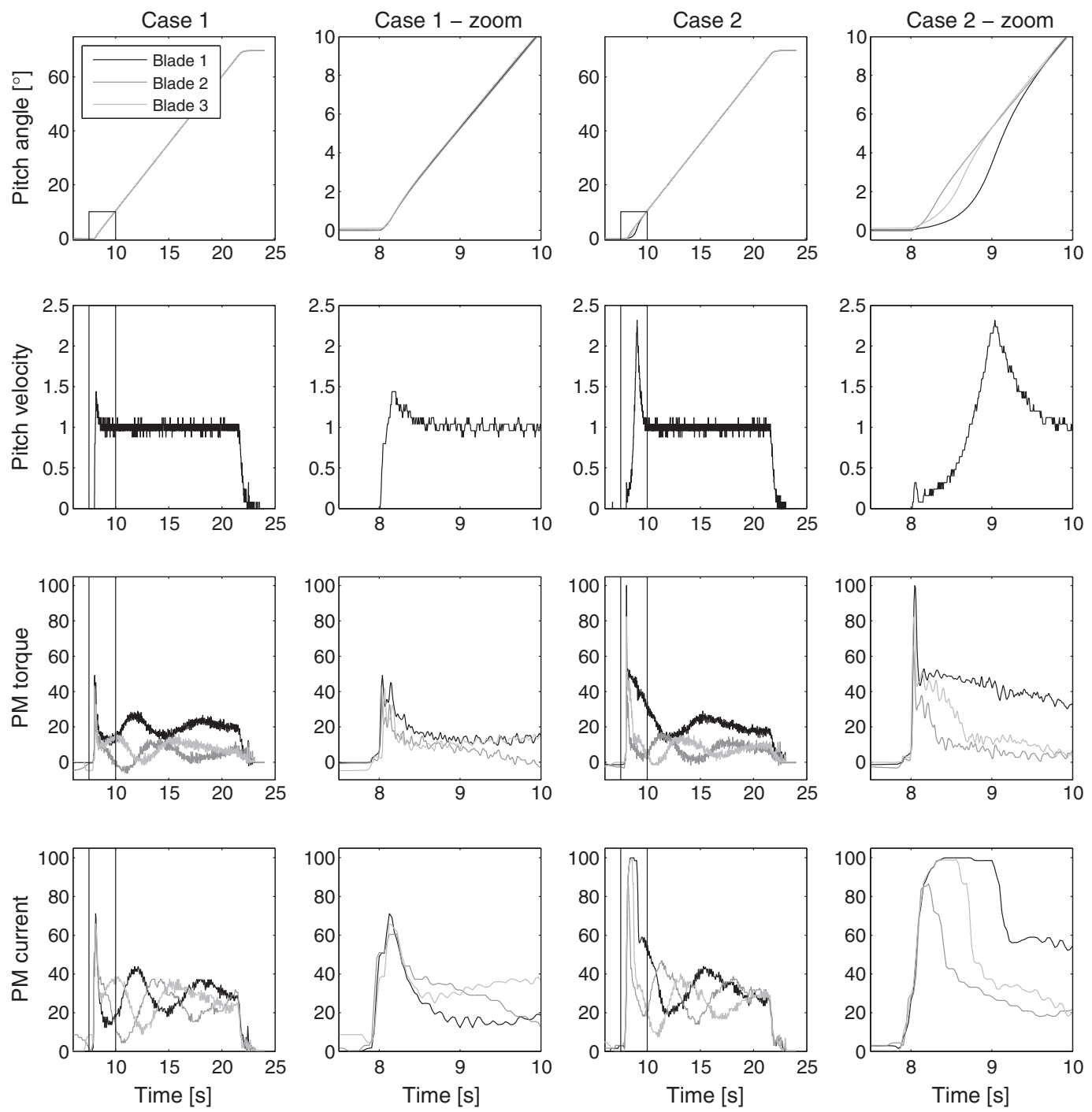

Figure 4. Measured pitch angle, pitch speed, PM torque and PM current for two cases and zoomed in and out. The zoom area is shown with a black box. 
maintenance, because the same thing happens in all time series of this kind, and the dependence of wind speed is eliminated, because it always happens at low wind speeds around $2-6 \mathrm{~m} \mathrm{~s}^{-1}$. This operational mode will be examined further in the following sections:

- Measurements from running to idling state

- Comparison between model and measurements

- Fitting of model to measurements

- Factors influencing maximum PM torque

- Analysis of relationship between PM current and torque

\subsection{Measurements from running to idling state}

In Figure 4, two time series are shown of pitch angle, pitch velocity, PM torque and PM current. Time series corresponding to the full range of motion of the blades from zero to $70^{\circ}$ are shown (columns 1 and 3 ) together with a magnification of the peaks in the beginning of the time series (columns 2 and 4). Case 1 has a low peak PM torque compared with case 2.

Several observations can be made for case 1. Regarding the velocity, there is a small overshooting of velocity in the beginning before the constant velocity is reached. The PM torque and current follow each other to some extent, but there are some differences. Where the current is at the same level during the time series for the three blades, the torque is significantly larger for blade 1 . Also, the initial peaks are different for torque and current, as the maximum of the torque comes before the maximum of the current. The torque has two peaks of almost same height, whereas the current has only one peak.

When case 2 is observed and compared with case 1, the peak PM torque is much larger. Furthermore, the overshooting of the velocity is larger, and it takes a relatively long time to accelerate to maximum velocity. This means that the pitch angle is clearly lacking behind the target position, especially for blade 1 . Furthermore, the first torque peak is significantly larger than the following torque peaks. The current peak comes later than the torque, and the current stays at maximum for a longer time.

An investigation of the cause of these variations between the two cases is relevant. Furthermore, it is important to see how well the measurements compare with the model and how well the PM current and torque are correlated. This will be examined in the following sections.

\subsection{Comparison between model and measurements}

On the basis of aeroelastic simulations in PHATAS, the PM torque has been calculated using equation 1 . The contributions from the separate terms and the total PM torque are shown in Figure 5 for one blade. The friction was calculated using equation 3 . As the figure shows, the moment is dominant, and therefore, equation 2 gives almost the same result as
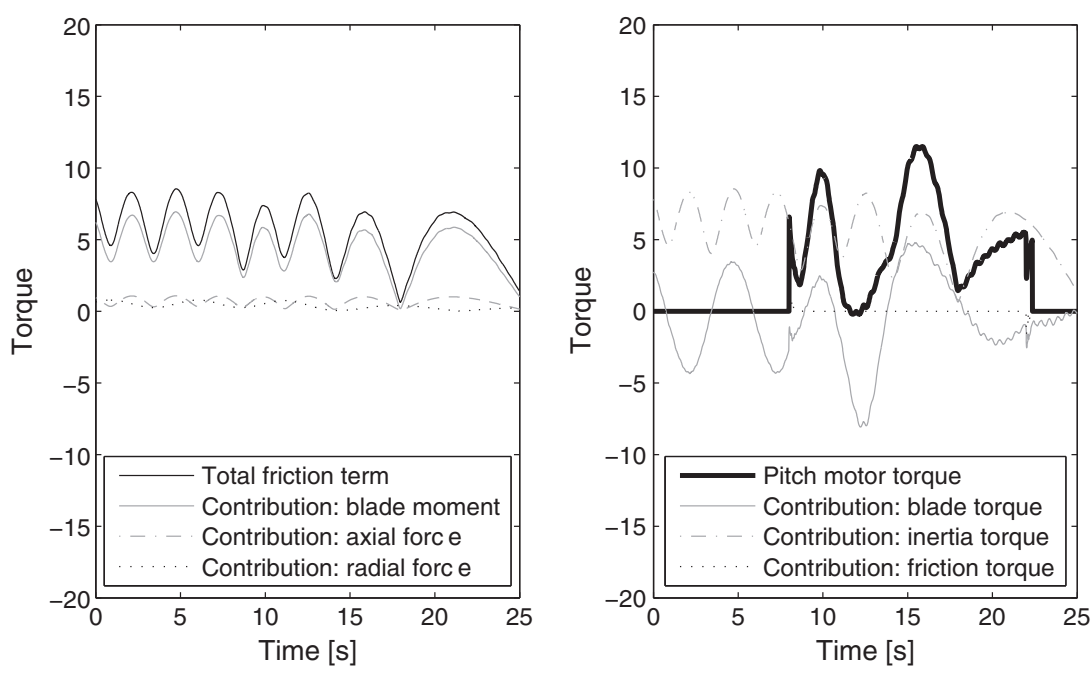

Figure 5. Model estimate of bearing friction and PM torque when the turbine is going from running to idling. The individual terms and the total torques are shown. 
equation 3 because only the term for radial load differs. The most important things affecting the PM torque in the model are the blade torque and moment.

In Figure 6, the measurements from case 1 are shown together with the model. The model does not fit very well to the measurements, which is why an adjustment of the models has been attempted. The adjusted models are also shown in Figure 6. To begin with, it is examined whether inaccuracies in the modeled moments cause the deviation. In Figure 7, the azimuth angle and blade moments for one of the blades are shown. The main contributor to the blade moment is the gravity. The modeled moments are similar to the measured moments, but the rotor decelerates more slowly than foreseen by the model. This gives an increasing time lag between the model and the measurements. To reduce this effect on the model, the measured moments were used instead of the moment from the aeroelastic model. This did not give a very good fit either. Therefore, the friction term was removed from the model and that gave the right shape, but the PM torque is generally too small. If a constant friction term (different for each blade) is then added to make a better fit, there is better agreement
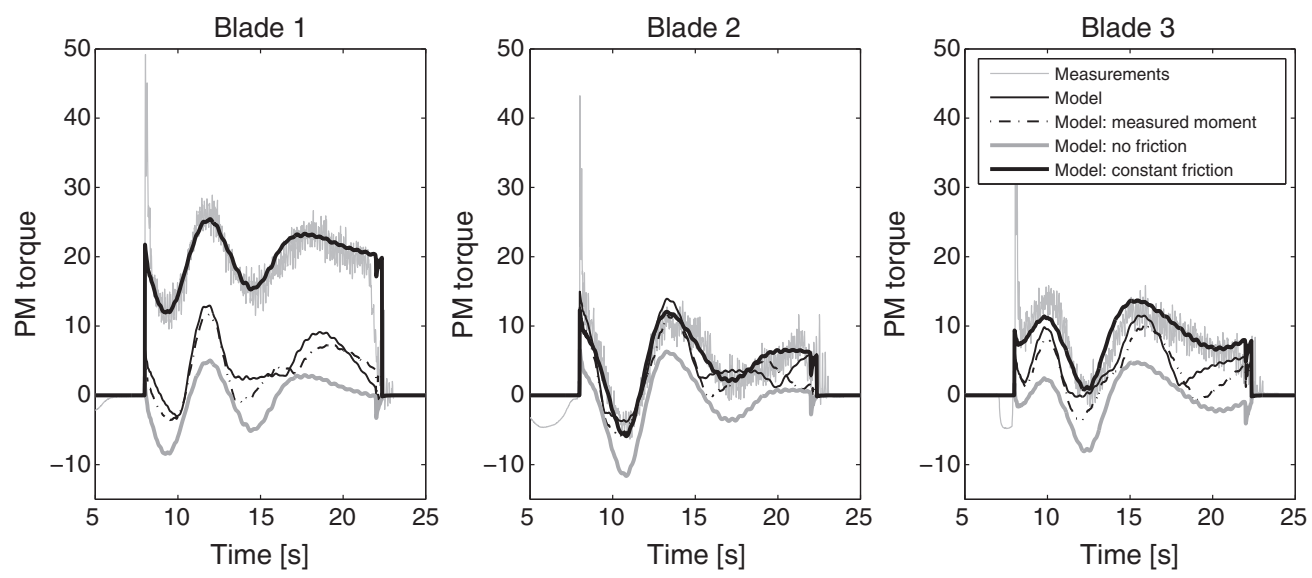

Figure 6. Comparison between model and measurements for PM torque.
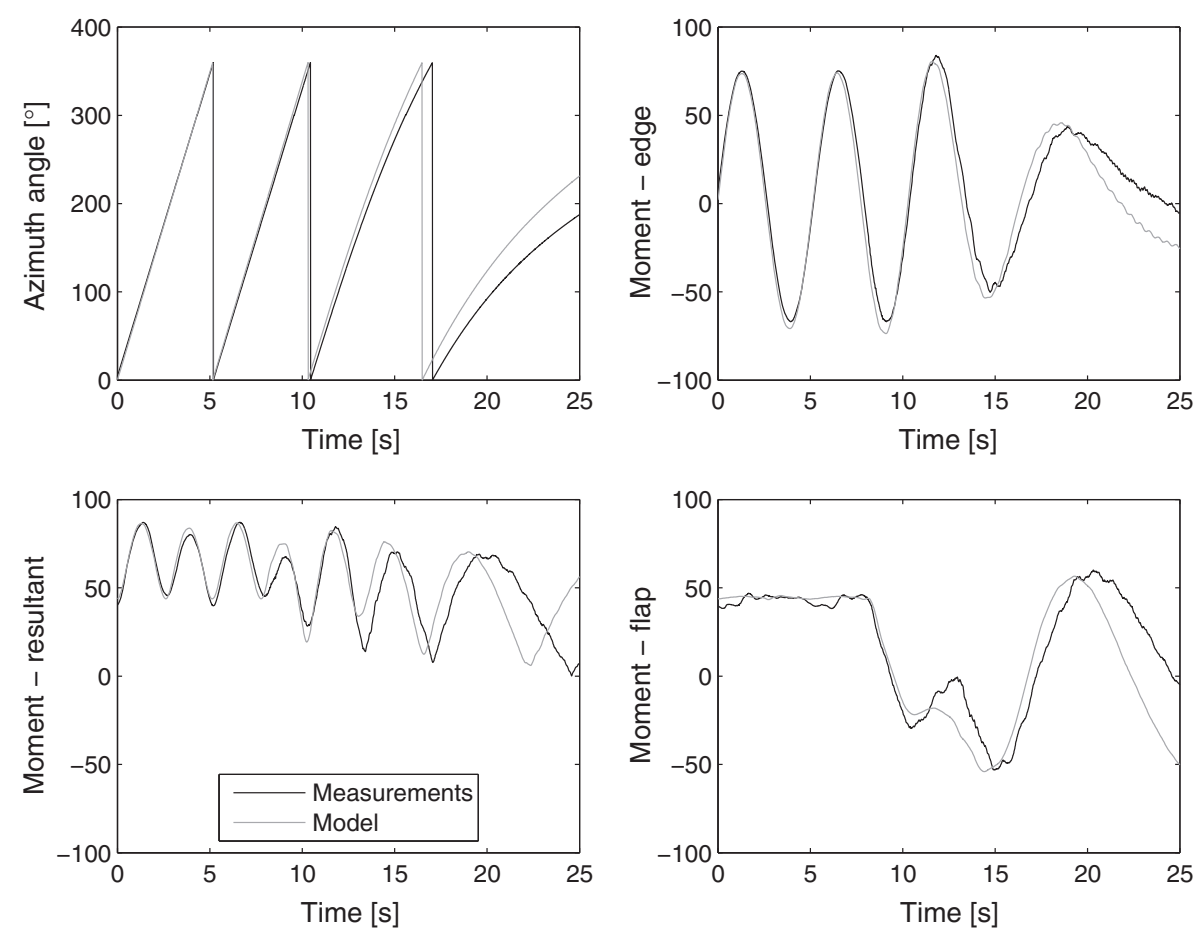

Figure 7. Comparison between model and measurements for blade moments. 
between measurements and model. The fact that the constant is different for the three blades indicates that the dynamic friction is different for the blade bearings in the three blades. However, Figure 4 showed that the PM current was of the same size for all three blades, and this makes it more probable that some kind of measurement error caused the deviations.

\subsection{Fitting of model to measurements}

The model with constant friction explains the shape of the PM torque to a certain extent, but the peak in the beginning is not explained. Figure 8 shows that the acceleration and velocity from the model do not equal the measurements. This could be caused by inaccuracies in the pitch control model, but it could also be due to loads not taken into account in the aeroelastic model. The measured PM torque has the same peaks as the acceleration; thus, the acceleration seems to be the cause of these peaks. The deviations between model and measurements with respect to the acceleration could possibly explain some of the differences.

A linear regression model has been created to investigate whether a better model can be obtained. The blade torque and moment are included, and a term proportional to the measured acceleration is added. Furthermore, a constant term and a term proportional to the velocity is included, as the friction for lubricated bearings can increase with velocity in some cases. ${ }^{12}$ These two terms have constants that can be different for the three blades, to account for the different offsets of these blades. The PM torque for blade $j$ at time point $t$ is assumed to be found by the following linear combination with constants $c$ and error $\varepsilon$ :

$$
T_{\mathrm{m}}(t, j) i_{\mathrm{gbx}} i_{\text {gear }}=c_{1}\left(T_{\mathrm{b}}(t, j)-I_{\mathrm{bl}} \ddot{\theta}_{\text {model }}(t, j)\right)+c_{2} I \ddot{\theta}(t, j)+c_{3} \frac{4.4 \mu}{2} M(t, j)+c_{4}(j)+c_{5}(j) \dot{\theta}(t, j)+\varepsilon
$$

The first three terms originate from the original model in equation 1 . The $c_{3}$ term originates from the friction model in equation 3. Only the moment is included because it is dominant, and the term $\frac{\dot{\theta}}{|\dot{\theta}|}$ is substituted with 1 because the velocity does not change direction. The constant $\frac{4.4 \mu}{2}$ is maintained such that the expected value of $c_{3}$ because of the original model is 1 .
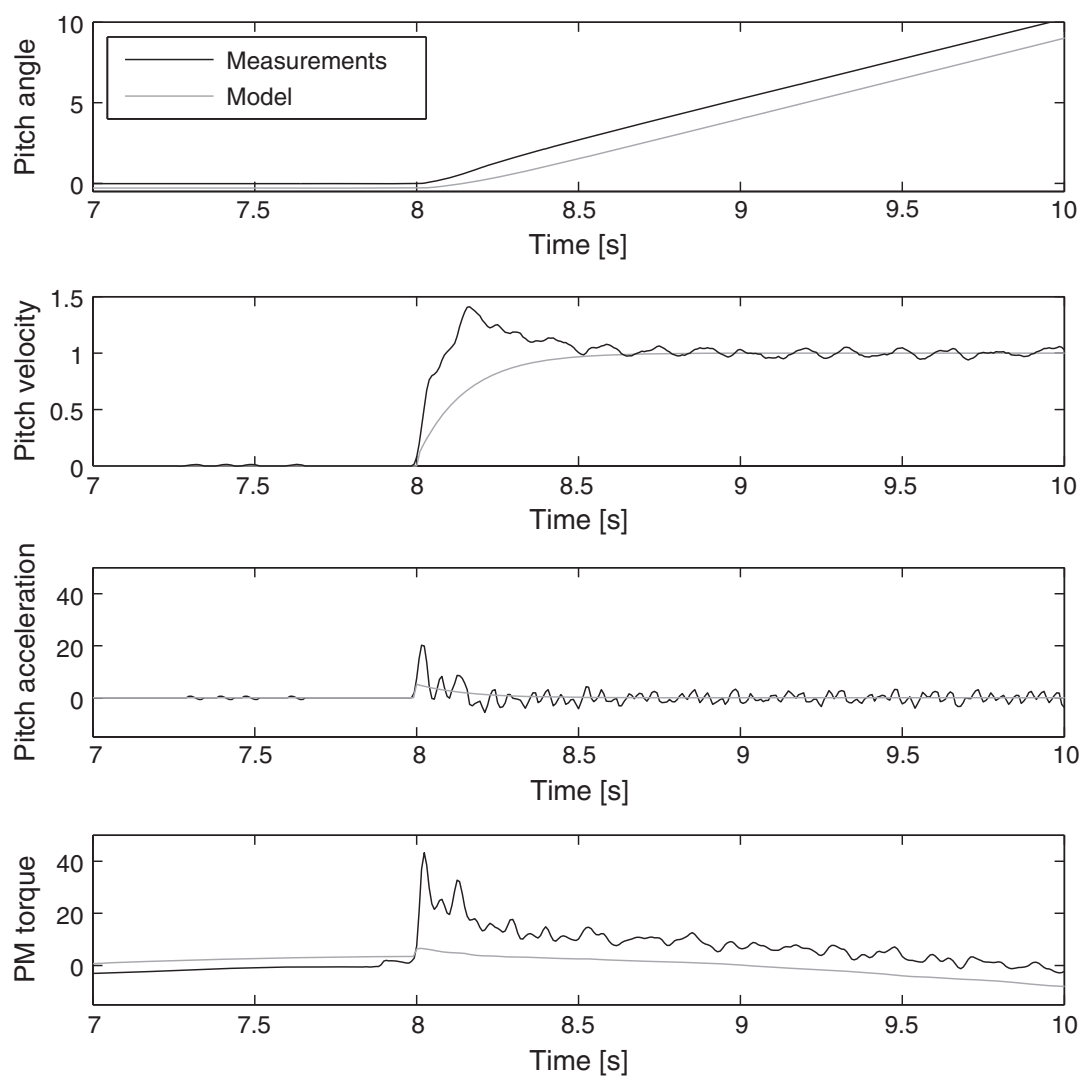

Figure 8. Comparison between model and measurements. 

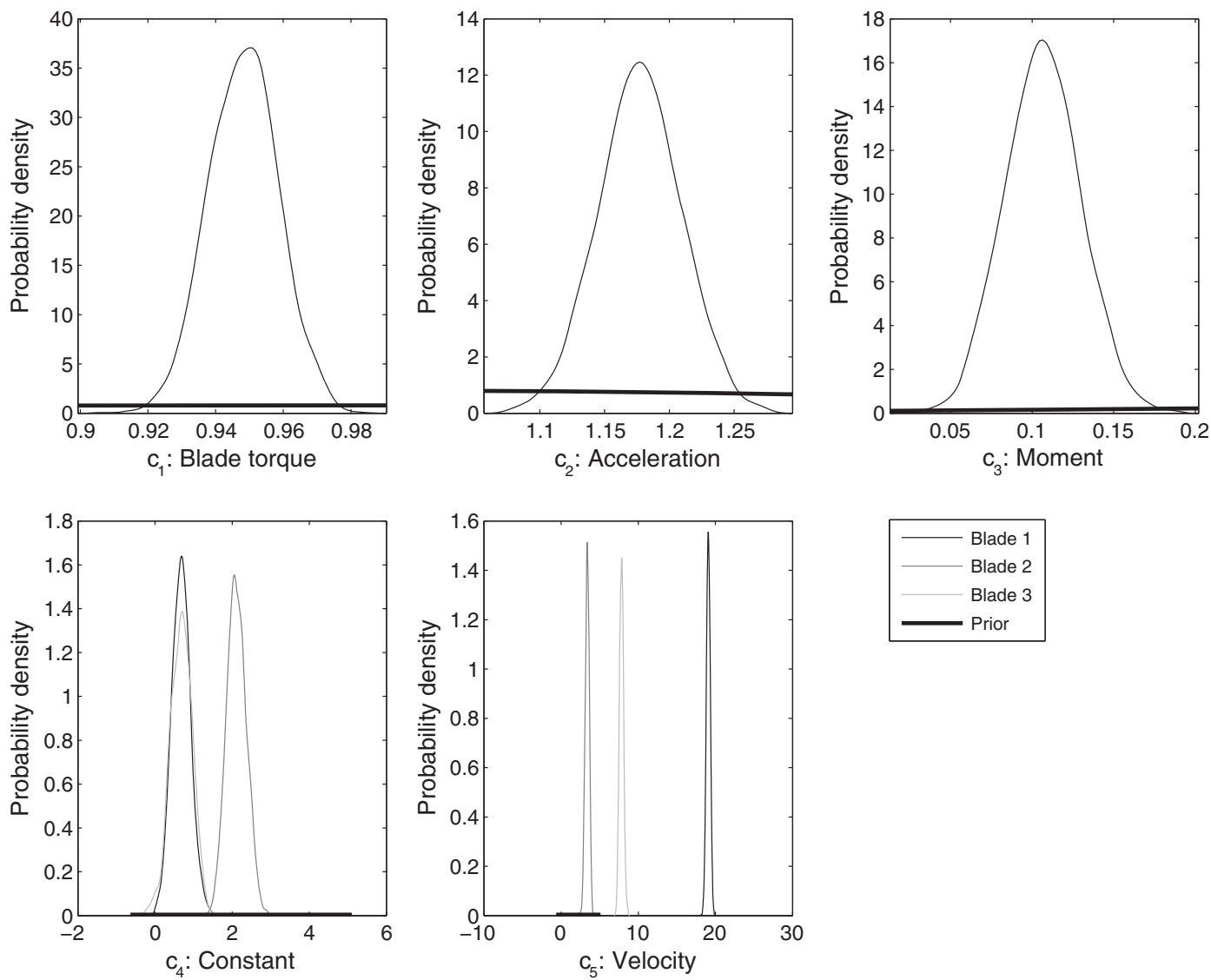

Figure 9. Posterior distributions for constants

A term proportional to the acceleration from the model is subtracted from the blade torque in the $c_{1}$ term because the blade inertia was included in the model blade torque $T_{\mathrm{b}}$ from PHATAS. The model acceleration, $\ddot{\theta}_{\text {model }}$, is however different from the measured acceleration, $\ddot{\theta} . I_{\mathrm{bl}}$ actually depends on deflection, but here approximated by a constant and estimated by comparing $T_{\mathrm{b}}$ and $\ddot{\theta}_{\text {model }} I$ is the total inertia of the masses being accelerated and equals $I_{\mathrm{bl}}+\left(i_{\mathrm{gbx}} i_{\text {gear }}\right)^{2} I_{\mathrm{gbx}}+I_{\mathrm{b}}$. For the resultant blade moment, $M$, and the velocity, $\dot{\theta}$, the measured values are used. The velocity is scaled to have a normal pitching speed of 1 so that the influence of $c_{4}$ and $c_{5}$ can be easily compared.

The model was made in the program 'OpenBugs' (Thomas Andrew, University of Helsinki, Finland), a program for Bayesian analysis of complex statistical models using Markov Chain Monte Carlo techniques. ${ }^{13}$ Here, the regression analysis is made with a Bayesian approach, where prior distributions can be specified for the constants, and the data is used to compute posterior distributions for the constants. The priors can have very narrow distributions if the uncertainties are small or can be very wide, which makes them 'non-informative', and the mean value of the prior will not affect the results.

All priors are assumed to be normally distributed. The terms $c_{1}$ to $c_{3}$ have priors with a mean value equal to one because they are from the original model. The standard deviations are set to 0.5 to put some weight on the prior, but still allow the measurements to affect the terms. For $c_{4}, c_{5}$ and $\varepsilon$, the priors are made non-informative by setting their means to zero and standard deviations sufficiently large to 100 .

The posterior distributions are found for case 1 using OpenBugs and are shown in Figure 9. The value of the standard deviation of $\varepsilon$ is found at 4.376 . The term $c_{1}$ is found to be around $5 \%$ less than in the model, and the acceleration term is $18 \%$ larger than expected. The influence of the moment is only $11 \%$ of the model value. According to the model, most of the 'offset' can be explained by the term proportional to the velocity instead of a constant. In Figure 10, the contributions from the individual terms to the PM torque are shown. Here, the influence of the moment is clearly seen to be very small.

In Figure 11, the measurements are shown together with the model values found using the means of the constants, and further, the residuals are shown. The upper row is for case 1 (low peak PM torque). In the lower row, the measurements from case 2 (high peak PM torque) are used together with the constants found in case 1. For case 1, the residuals are 

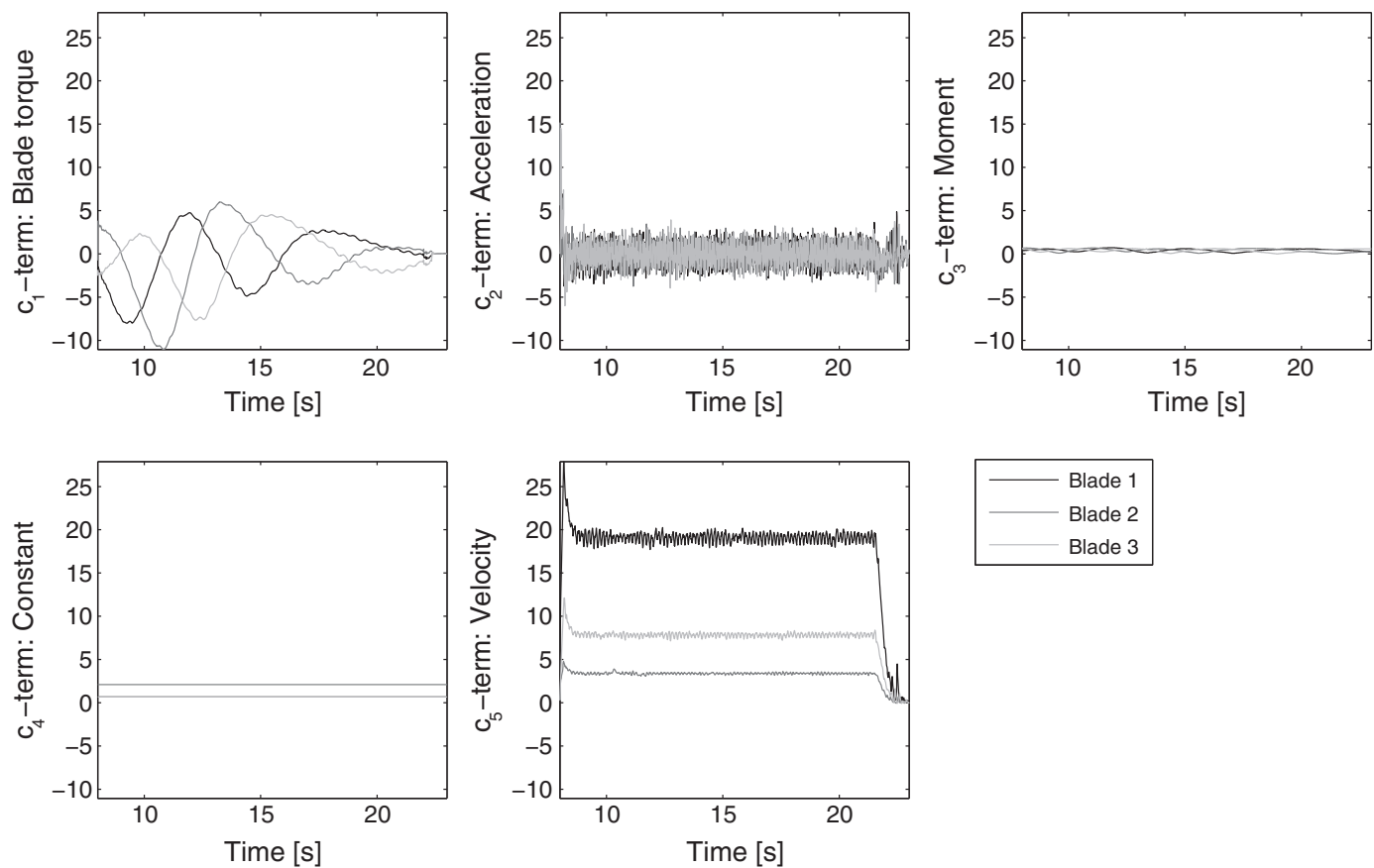

Figure 10. Contributions from the individual terms.
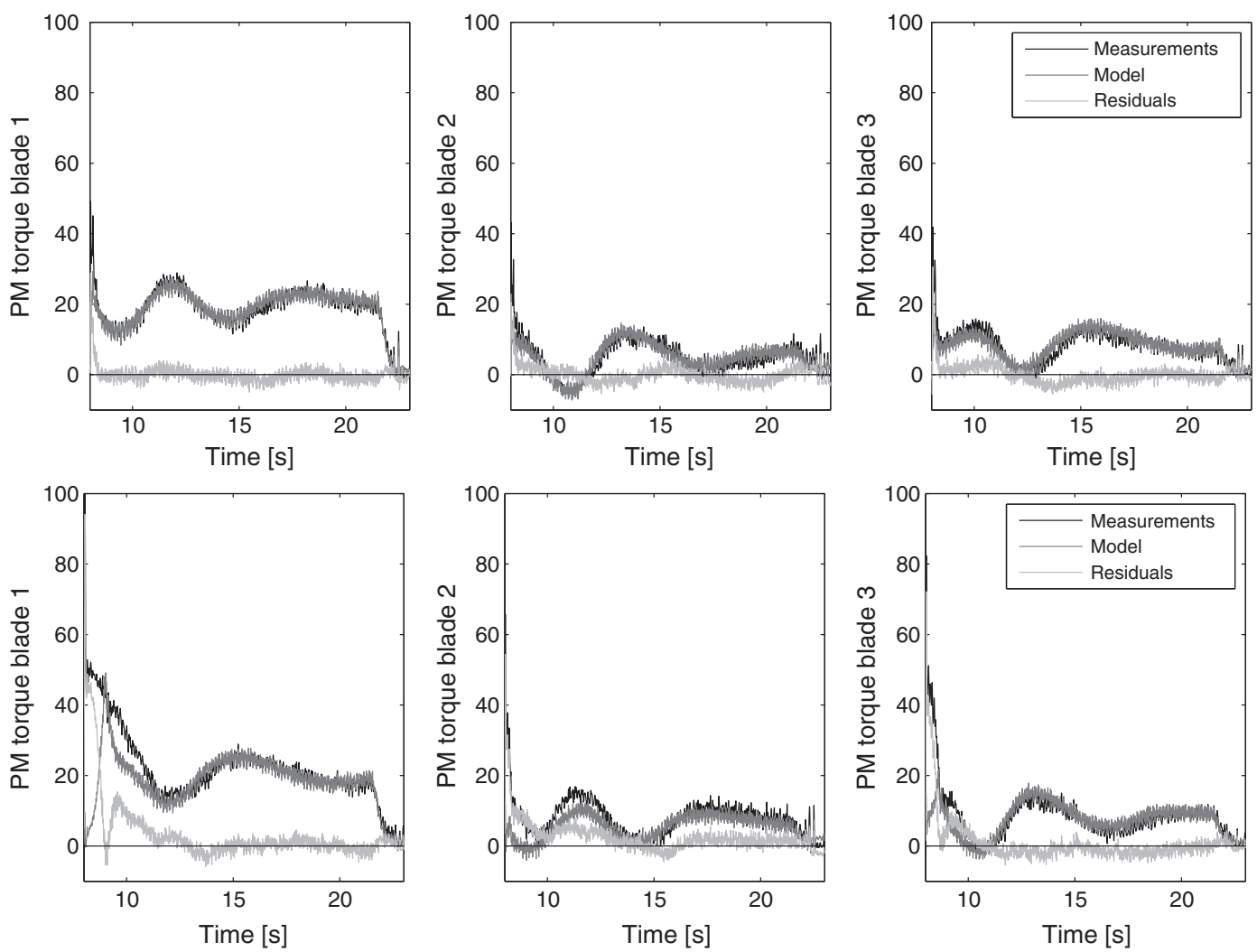

Figure 11. Measurements, model results found using mean values of the posterior distributions and residuals. The three upper figures are for case 1, and the three lower figures are for case 2, with constants found using case 1. 
generally rather small, except for the peak in the very beginning. For case 2, on the other hand, the residuals are larger on a longer distance near the peak, especially for blade 1 . Here, the overshooting in velocity does not match the measured PM torque. The main reason that the velocity dependent term is preferred over the constant term seems to be that it follows the reduction of PM torque near the end.

\subsection{Factors influencing maximum PM torque}

The regression analysis presented in the previous section showed that the peak PM torque in the beginning could not be explained by terms proportional to acceleration, velocity or moment. As shown in Figure 2, there is a large difference between the maximum values for different time series. The peaks are well defined, and therefore, it is not expected that the difference in the size of the peaks are caused by measurement inaccuracies. Neither can it be caused by over-modulation of the pitch controller, as it would not explain the difference between time series. Instead, the pitch velocity increases more slowly for larger peak torques, as shown in Figure 4, and the peak is more likely caused by static friction in the blade bearing. In this section, it will be examined whether factors influencing the peak PM torque can be found. Status change from running to idling always happens at low wind speeds, and therefore, the wind speed and turbulence do not influence the PM torque.

First, it is investigated whether the size of the peak is influenced by the azimuth angle at the time pitching starts. In Figure 12, the peak value of the PM torque is shown as a function of azimuth angle for all three blades for 39 cases. For comparison, the theoretical influence of the azimuth angle from equation 1 is also shown. When the azimuth angle is $90^{\circ}$, both the blade torque and moment have their maximum value, and therefore, this gives the maximum PM torque according to the theoretical model. However, the measurements do not seem to have the same dependence of the azimuth angle, and the variation between measurements is much larger than the variation in blade torque and moment.

Other factors potentially influencing the bearing friction are factors related to the lubrication. One possible influencing factor is the bearing temperature because the viscosity of the grease is affected by temperature. Another factor is the time passed since the blades were previously pitched (still time). In other words, the amount of time the blades have been positioned at a pitch angle of $0^{\circ}$, which happens during normal production below rated wind speed. When the still time is long, the blade has been oscillating back and forth with small amplitude for a long time. This can cause the grease to be unevenly distributed in the bearing and could make the PM torque required for startup larger.

To investigate this, $10 \mathrm{~min}$ statistics have been used from the entire measurement period. The maximum values of PM torque and current in the time intervals, where the turbine status changed from running to idling, were compared on the basis of the mean bearing temperature in the time interval and the still time. In Figure 13, the maximum PM torque and current are shown as function of time, still time and temperature. The figures show that the PM current and torque are larger at smaller temperatures and for longer still times. The dependence of temperature seems to be most clear for the PM current.

The dependence on still time explains the difference between cases 1 and 2, as the still time is much larger for case 2 .

\subsection{Analysis of relationship between PM current and torque}

In order to use the PM current for condition monitoring, it is necessary to understand the relationship between PM current and torque. In Figure 4, the PM torque and current do not follow each other accurately, especially near the peak. In fact,

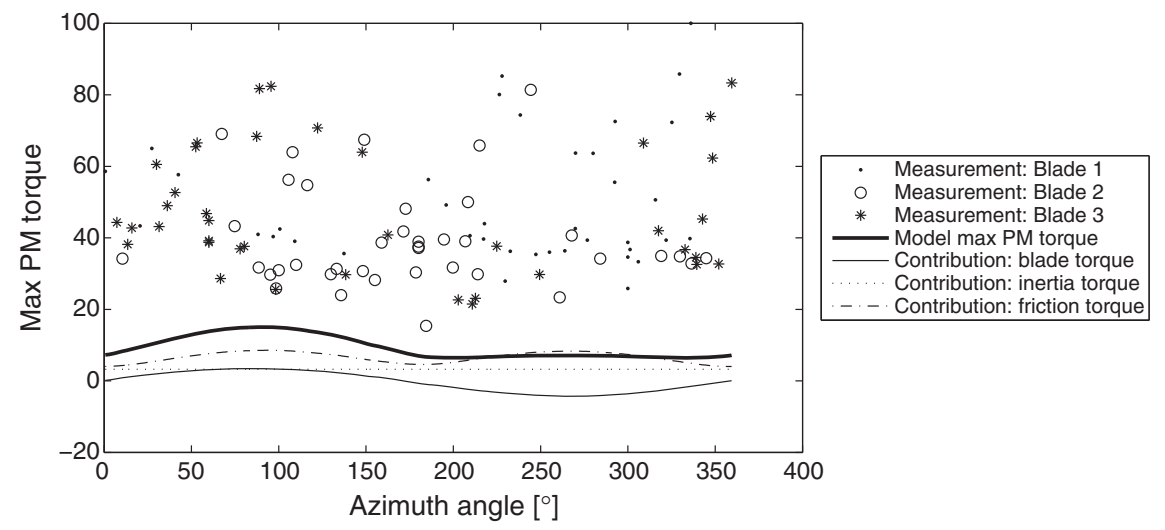

Figure 12. Peak PM torque as function of azimuth angle for model and measurements. 

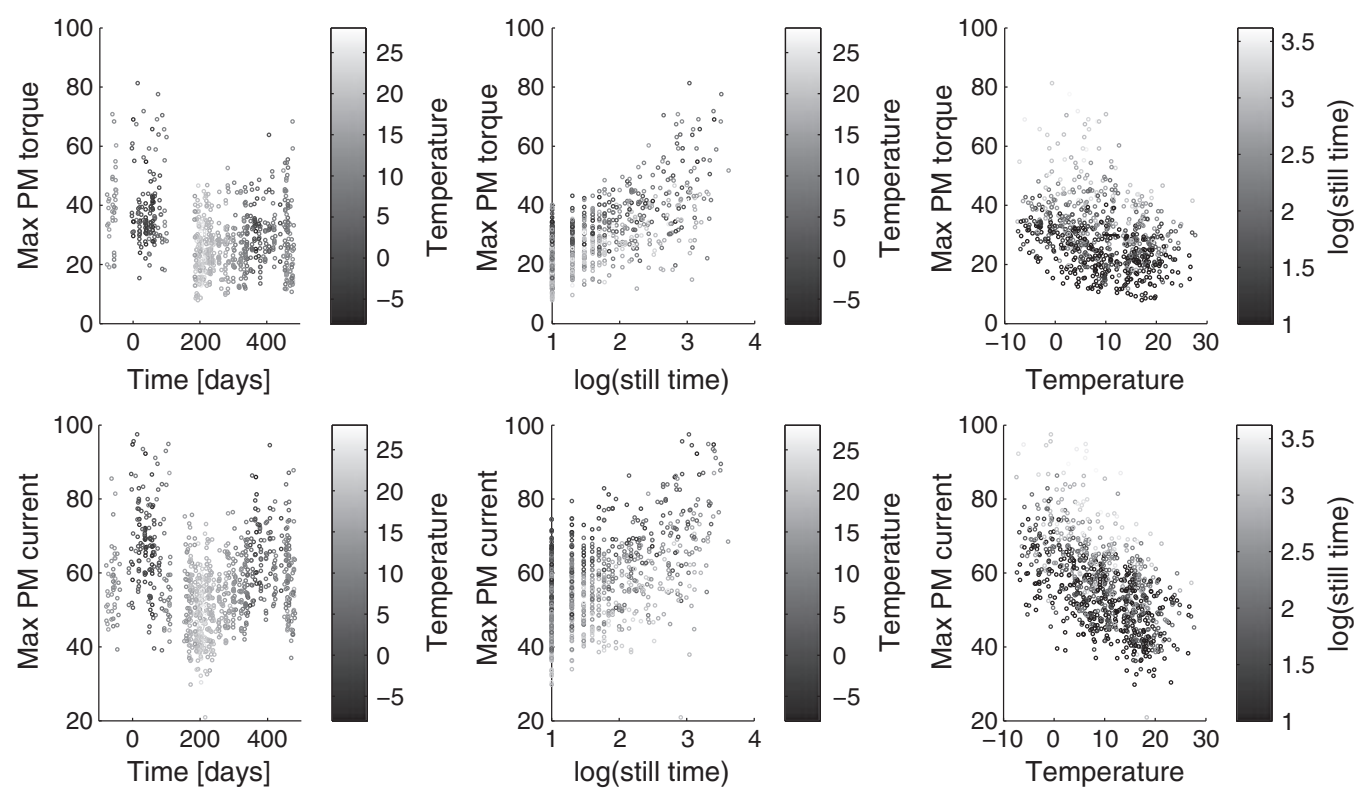

Figure 13. Influence of time since last pitching and temperature on PM current and torque. Blade 2.
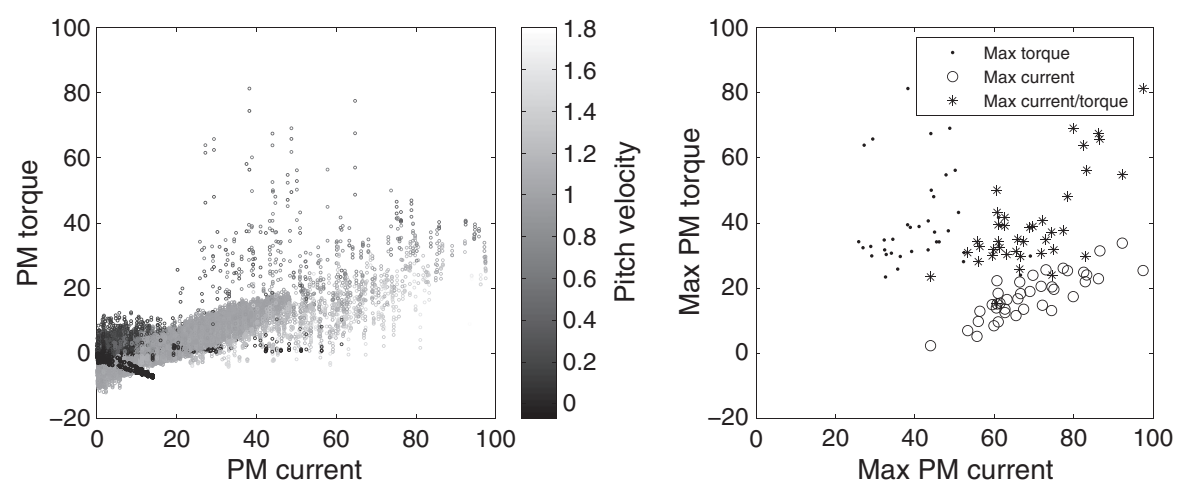

Figure 14. Proportionality between values of PM current and torque for 39 time series. Blade 2.

the PM current is rather low at the time of the maximum PM torque. The PM current is expected to be proportional to the electrical PM power, and the mechanical PM power is the product of the PM torque and speed. Therefore, the PM torque and current are expected to be proportional, but only at constant pitching speed. Furthermore, the power efficiency factor might depend on the speed or torque, and a motor with high efficiency at low speeds is suitable for applications like this with large startup torque.

Figure 14 (left) shows a scatter plot of PM torque as function of the PM current for the data points in 39 time series, and the shades show normalized pitching velocity, where one is the velocity during normal pitching. When the normalized velocity is close to one, there is proportionality between PM torque and current with good approximation. At higher velocities, the torque delivered at a specific current level is smaller, and at lower velocities, the torque delivered is larger. Furthermore, there is a population around zero velocity and zero toque, which originates from the part of the time series where the pitch system is not attempting to turn the blades yet/anymore.

For a condition-monitoring system, it can be an advantage if only the statistics were necessary to use and not the entire time series. Therefore, it is investigated whether there is approximate proportionality between the maximum values. In Figure 14 (right), three different sets of values are compared; values from the time the PM torque is maximum, values from the time the PM current is maximum and the maximum values of PM torque and PM current. There is approximate proportionality between the values at maximum PM current, but at maximum PM torque, there are many outliers. However, there is approximate proportionality between the two maximum values, even though they do not occur at the same time. 


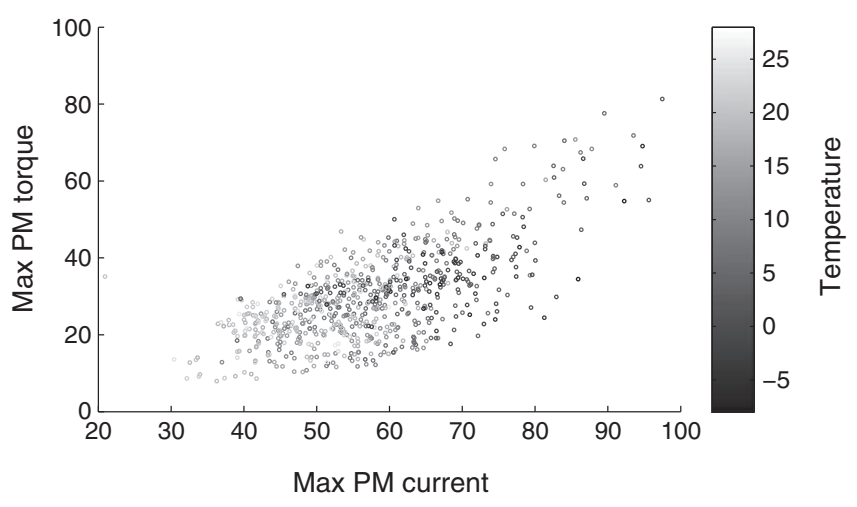

Figure 15. Proportionality between maximum values of PM current and torque and the dependence of temperature. Blade 2 .

The reason for this can be found by considering what is happening in the controller when the pitching starts. When the friction is high, the initial torque peak is high, which causes low velocity. This makes it necessary to increase the velocity so as to catch up, which gives an increased inertia (acceleration) contribution to the torque. This increased PM torque and high speed increase the PM current at this second peak, where the maximum PM current typically occurs.

In Figure 15, the maximum values of PM current and torque are shown for the entire measurement period together with the temperature. For a large maximum PM torque of a certain value, the maximum PM current is larger if it is colder. This indicates that the temperature affects the second peak more than the first one, which is more affected by stand still time.

\section{CONCLUSIONS}

In this paper, measurements of PM torque and current were compared with a theoretical model, and potential factors influencing the measurements were analyzed. The blade moment was found to have only minor influence on the blade bearing friction, even though it has an influence according to the theoretical model. A more accurate fit could be obtained by omitting the friction term dependent on blade forces and include a constant or velocity dependent term instead. The term proportional to the velocity was preferred by the model, because the PM torque was decreasing with the velocity at the end of the time series. However, in the beginning of the time series, the velocity-dependent term gave a less accurate fit. The peak in the beginning was larger than the inertia forces could explain, and this is most likely due to static friction in the blade bearing. It was found not to be influenced by the azimuth angle but instead to be influenced by temperature and time passed since the blades were previously pitched.

The PM current and torque are only proportional at constant velocity, and the maximum values of torque and current occur shortly after the pitching starts, when the velocity is not yet constant. Furthermore, the torque peaks before the current. However, the maximum values of current and torque are still approximately proportional.

As no damages in the pitch bearings were detected during the measurement period, it is not possible to determine if the PM current can be used to detect it. However, this analysis gives a very useful starting point for further analyses.

First, 10 min statistical values of torque or current can most likely not be used to predict increased bearing friction caused by damages, because of the strong influence of time since last pitching and temperature and because of additional uncertainties probably originating from varying lubrication coverage.

Instead, the analysis should be based on time series. The first seconds of pitching after a long time with no pitching could be discarded from the analysis. This is because this part of the time series seems to be affected by the same factors also affecting the maximum values, in addition to the changes in velocity and acceleration when it starts pitching.

An approach would be to only use the part of the time series with an almost constant pitching speed, as this would decrease the number of variables needed in the model and would improve proportionality between PM current and torque. Still, it should be taken into consideration that the PM torque would be affected by azimuth angle, temperature, pitch angle and minor acceleration oscillations, in addition to increased friction from damages.

\section{ACKNOWLEDGEMENT}

The work presented in this paper is part of the project 'Reliability-based analysis applied for reduction of cost of energy for offshore wind turbines' supported by the Danish Council for Strategic Research, grant no. 2104-08-0014. The financial support is greatly appreciated. 


\section{REFERENCES}

1. Fraunhofer Institute for Wind Energy and Energy System Technology (IWES). Wind Energy Report Germany 2010, Kassel, Germany, 2011. [Online]. Available: http://windmonitor.iwes.fraunhofer.de/wind/download/Windenergie_ Report_2010_en.pdf. (Accessed July 2012).

2. Sørensen JD. Framework for risk-based planning of operation and maintenance for offshore wind turbines. Wind Energy 2009; 12: 493-506. DOI: 10.1002/we.344.

3. Farrar CR, Duffey TA, Doebling SW, Nix DA. A statistical pattern recognition paradigm for vibration-based structural health monitoring. In Presented at the 2nd International Workshop on Structural Health Monitoring. Technomic Publishing: Lancaster, Pennsylvania, 1999; 764-773.

4. Huston D. Structural Sensing, Health Monitoring, and Performance Evaluation. Taylor \& Francis: Boca Raton, Florida, 2010. DOI: 10.1201/b10369-4.

5. Nielsen JJ, Sørensen JD. Analysis of pitch gear deterioration using indicators, Proceedings of the EWEA Offshore Conference 2011, Amsterdam, Holland, 2011.

6. Perišić N, Pedersen BJ, Kirkegaard PH. Blade bearing friction estimation of operating wind turbines. Conference Proceedings of the Society for Experimental Mechanics Series 2012; 6: 213-220.

7. Holierhoek JG. PROTEST Final Report. ECN-E-10-100, Energy Research Centre of the Netherlands, Petten, Holland, 2010. [Online]. Available: http://www.protest-fp7.eu/fileadmin/protest/user/documents/D19_Final.pdf. (Accessed July 2012).

8. Harris T, Rumbarger JH, Butterfield CP. Wind Turbine Design Guideline DG03: Yaw and Pitch Rolling Bearing Life. NREL/TP-500-42362, National Renewable Energy Laboratory, Golden, Colorado, 2009. [Online]. Available: http://www.nrel.gov/docs/fy10osti/42362.pdf. (Accessed July 2012).

9. Rothe Erde Slewing Bearings, ThyssenKrupp, Dortmund, Germany, 2007. [Online]. Available: http://www.rotheerde. com/download/info/V01A_RE_GWL_GB_W.pdf. (Accessed July 2012).

10. Savenij FJ, Korterink H, Pieterman RP, van de Braam H, Engels WP, Baehr S. PROTEST Case study 2: Pitch system. ECN-X-10-119, Energy Research Centre of the Netherlands, Petten, Holland, 2010.

11. Holierhoek JG, Lekou DJ, Hecquet T, Søker H, Ehlers B, Savenije FJ, Engels WP, van de Pieterman RP, Ristow M, Kochmann M, Smolders K, Peeters J. PROcedures for TESTing and measuring wind turbine components; results for yaw and pitch system and drive train. Wind Energy 2012; Article in Press. DOI: 10.1002/we.1511.

12. Rolling Bearing Lubrication. WL 81 115/4 EA, Schaeffler Technologies AG \& Co, KG, Germany, 2002. [Online]. Available: http://www.schaeffler.com/remotemedien/media/_shared_media/08_media_library/01_publications/ schaeffler_2/publication/downloads_18/wl_81115_4_de_en.pdf. (Accessed July 2012).

13. Lunn D, Spiegelhalter D, Thomas A, Best N. The BUGS project: evolution, critique, and future directions. Statistics in Medicine 2009; 28: 3049-3067. DOI: 10.1002/sim.3680. 